Seton Hall University eRepository@Seton Hall

Summer 8-2011

\title{
Attenuated Total Reflection Infrared Spectroscopy (ATR-IR) as an In Situ Technique for Dissolution Studies
}

Abe S. Kassis

Seton Hall University

Follow this and additional works at: https://scholarship.shu.edu/dissertations

Part of the Biochemistry Commons

\section{Recommended Citation}

Kassis, Abe S., "Attenuated Total Reflection Infrared Spectroscopy (ATR-IR) as an In Situ Technique for Dissolution Studies" (2011).

Seton Hall University Dissertations and Theses (ETDs). 1432.

https://scholarship.shu.edu/dissertations/1432 


\section{Attenuated Total Reflection Infrared Spectroscopy (ATR-IR) as an In Situ Technique for Dissolution Studies}

by

Abe S. Kassis

\section{Ph.D. DISSERTATION}

Submitted in partial fulfillment of the requirements for the degree of Doctor of Philosophy in the Department of Chemistry and Biochemistry of Seton Hall University

\section{Seton Hall University}

Department of Chemistry and Biochemistry

400 South Orange Avenue

South Orange, New Jersey 07079

August 2011 


\section{DISSERTATION COMMITTEE APPROVALS}

We certify that we have read this thesis and that in our opinion it is sufficient in scientific scope and quality as a dissertation for the degree of Doctor of Philosophy

\section{APPROVED BY:}

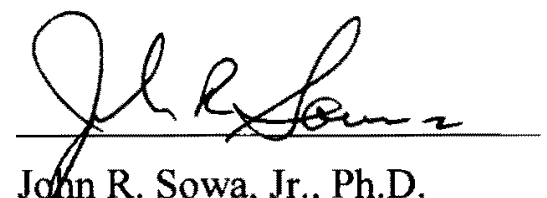

John R. Sowa, Jr., Ph.D.

Advisor, Seton Hall University

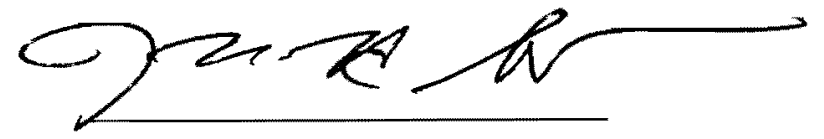

Nicholas H. Snow, Ph.D.

Member of Dissertation Committee, Seton Hall University

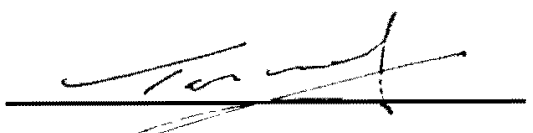

Tarun Patel, Ph.D.

Member of Dissertation Committee, Novartis Pharmaceuticals Corporation

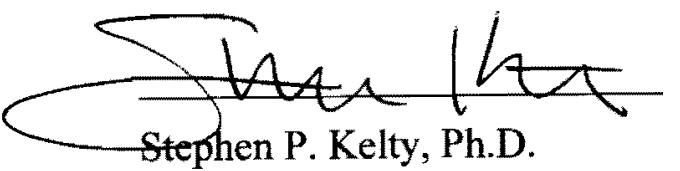

Chair, Department of Chemistry and Biochemistry, Seton Hall University 
"Although nature commences with reason and ends in experience it is necessary for us to do the opposite, that is to commence with experience and from this to proceed to investigate the reason."

-Leonardo da Vinci 


\section{Abstract \\ Attenuated Total Reflection Infrared Spectroscopy (ATR-IR) as an in situ Technique for Dissolution Studies}

Dissolution studies are critical tests for measuring the performance, or rate of release, of a drug product. There are many variables that affect the dissolution rate of the drug. Such variables may include characteristics of the active pharmaceutical ingredient (API) (e.g., particle size, crystal form, and bulk density), drug product composition (e.g., drug loading, and the identity, type, and levels of excipients), the drug product manufacturing process (e.g., compression forces, equipment), and the effects of stability storage conditions (e.g., temperature, humidity). Since dissolution has the ability to detect these variables, the regulatory agencies (i.e. FDA and EMEA) have placed great emphasis on the test. Moreover, the regulatory agencies have increased the monitoring and auditing of the dissolution test.

A novel technique using in situ attenuated total reflection-infrared spectroscopy (ATR-IR) to monitor dissolutions of pharmaceutical drug products was developed. The accuracy of this technique is $\pm 3 \%$ relative to HPLC. Salicylic acid calibrator tablets were studied and two over-the-counter (OTC) formulations containing acetaminophen, and/or acetylsalicylic acid were tested during the research. This novel approach was also used to analyze multiple components in a single tablet. For example, dissolution of the individual components of caffeine-free version of Excedrin $\circledast$ tablets, acetaminophen and acetylsalicylic acid were easily distinguished. 
Furthermore, the system has the capability of monitoring the drug transformations during dissolution. For instance, this technique was used to monitor the dissolution and hydrolysis of aspirin to salicylic acid. The ATR-IR system was found to have good sensitivity and can analyze samples as low as $0.03 \mathrm{mg} / \mathrm{mL}\left(2 \times 10^{-4} \mathrm{M}\right)$ based on the limit of detection ("LOD") studies. This will be discussed in details in chapter four. This technique allows deeper insight into spectroscopy, reaction analysis, kinetics and dynamics of the dissolution of active components in a pharmaceutical formulation.

In addition, this novel system was used to support research related to the BP oil spill that occurred in the Gulf of Mexico during April 20th - June 3rd, 2010. Specifically, the system was used to study dispersion of oil in the presence of dishwashing soaps as preliminary

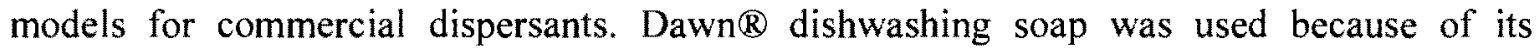
powerful claim to clean wildlife affected by oil spills. Dawn $\mathbb{B}$ specifically uses anionic surfactants, such as alkyl dimethyl amine oxide, to break down oil samples. These surfactants reduce the surface tension of water and can weaken the barrier that automatically forms between oil and water, allowing them to unnaturally mix.

Furthermore, in recognition of the potential of this technique to be widely used in the pharmaceutical industry for drug dissolution studies, an instrument was designed and the design is presented as a chapter in this dissertation. Overall, this work has discovered and validated the use of ATR-IR as a technique for monitoring to dissolution and dissolution/hydrolysis reactions of pharmaceutical formulations. It has excellent potential for studies of the dissolution of tablets especially multi-component tablets and tablets with ingredients that undergo subsequent hydrolysis. 


\section{Acknowledgments}

During my graduate career at Seton Hall University, I have had the privilege and opportunity to develop myself as a research scientist and strategic thinker. This would not have been possible without the support I received from my great employer Novartis Pharmaceuticals Corporation, for giving me the financial support and opportunity to obtain my doctorate degree. Moreover, I would like to give special thanks to Cynthia Cetani, Andre Wyss, Colleen Ruegger, Steffen Lang, Lynne Delisi and many others for their support and mentorship. Also, I would like to give special thanks to my research advisor Dr. John R. Sowa, Jr. for his valuable guidance, expertise, encouragement, and his dear friendship. Dr. Sowa inspires me to become a better scientist and individual.

I would also like to acknowledge Dr. Sowa's research group as well as the faculty members of the Department of Chemistry and Biochemistry at Seton Hall University. Specifically, I would like to give special appreciation to; Dr. Snow for encouraging, motivating and supporting my research ambitions, Dr. Kazakevich for his great guidance and teachings in HPLC, Dr. Kelty, Dr. Murphy, Dr. Fadeev and Dr. Marzabadi. I would also like to acknowledge Dr. Tarun Patel from the Novartis Pharmaceuticals Corporation for his guidance and mentoring throughout my studies. I will forever be indebted to those who have supported me through the years in this endeavor. It is with great appreciation that I offer my gratitude to all.

To my Dad and Mom, Sam and Nina Kassis for opening the door to science and motivating me to reach further in life. To my dear sisters, family and close friends. 
Finally, I want to thank my wife, Cindy for her unwavering support. Also, I want to thank my daughter Amber and my son Neil for their support and understanding. Without them none of this would have been possible.

I would like to acknowledge Wes Walker and Jane Riley from Mettler-Toledo for technical support for the $\mathrm{iC}_{10} \mathrm{IR}$ Instrument. 


\section{Table of contents}

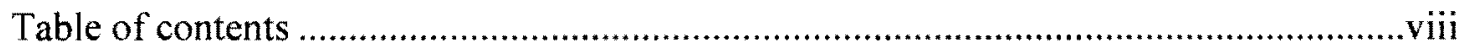

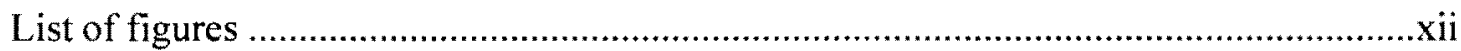

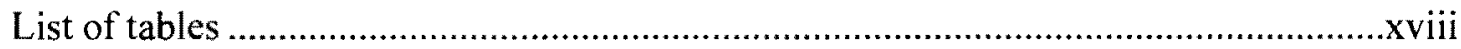

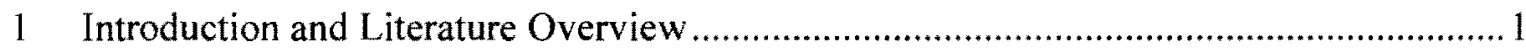

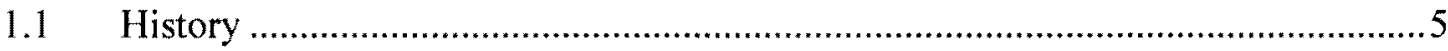

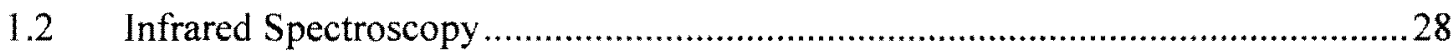

1.3 Literature background: in situ ATR-IR and dissolution ..................................32

2 Dissolution of salicylic acid, acetylsalicylic acid and acetaminophen using $I n$ Situ

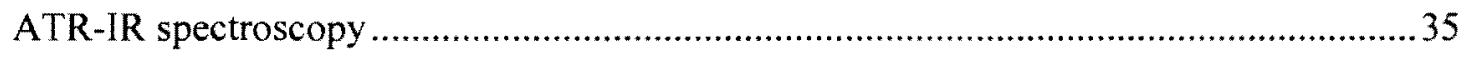

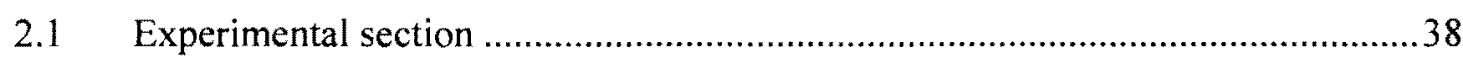

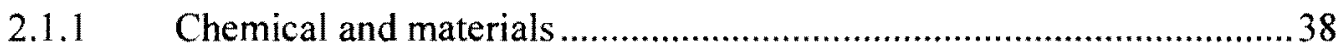

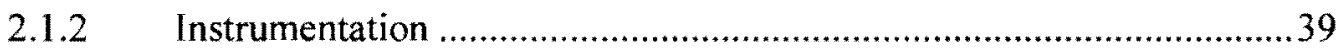

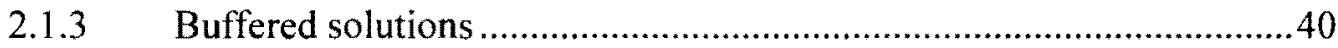

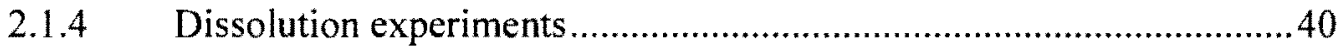

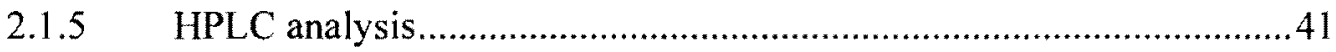

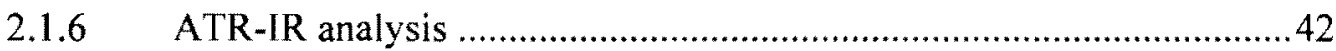

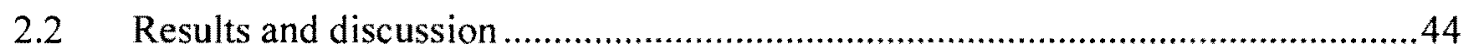

2.2.1 Single-component analysis .................................................... 44

2.2.2 Dissolution results for salicylic acid using $\mathrm{pH} 7.4$ phosphate buffer ...47 
2.2.3 Multi-tablet analysis.

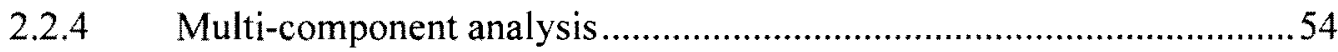

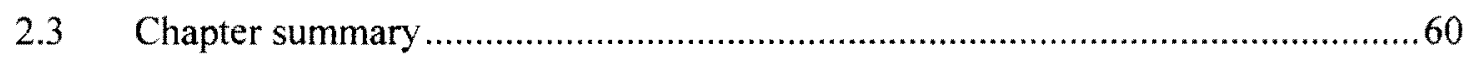

3 Kinetics of drug hydrolysis of common over-the-counter (OTC) drugs via ATR-IR spectroscopy 61

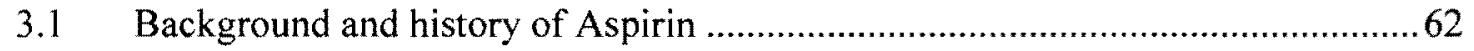

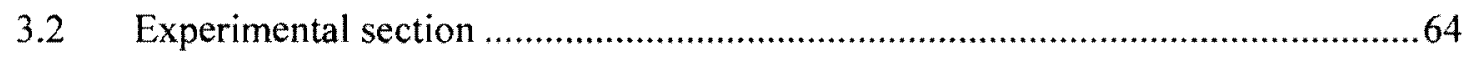

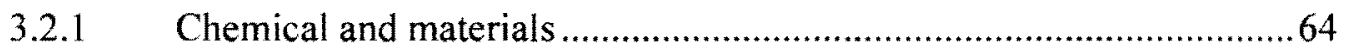

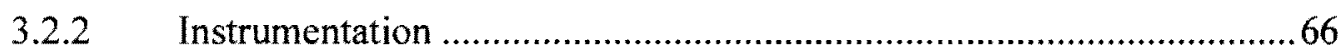

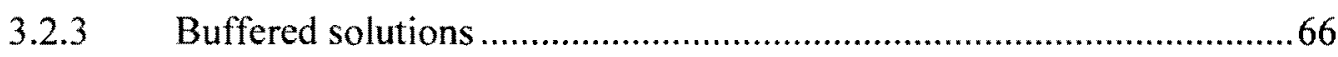

3.2.4 Hydrolysis Experiments ............................................................6. 67

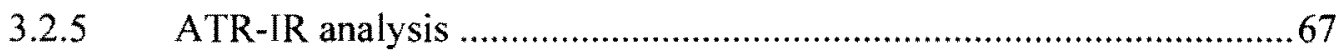

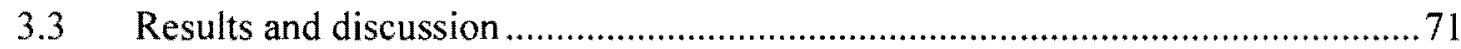

3.3.1 Hydrolysis of aspirin to salicylic acid in test tubes...........................71

3.3.2 Hydrolysis of aspirin to salicylic acid using aspirin tablet as the

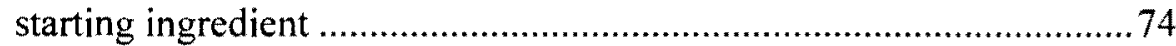

3.3.3 Hydrolysis of aspirin to salicylic acid using aspirin powder as the starting ingredient 77

3.3.4 Hydrolysis data: comparison between tablet and powder

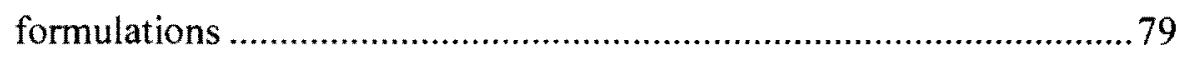

3.3.5 Kinetic rates observed during hydrolysis experimentation.................84 
3.4 Chapter summary

4 Instrument Development Proposal: "Solv-IR" as an In-Situ ATR-IR Instrument for

Solvation Chemistry 89

4.1 Background. 89

4.2 Broader impacts from the proposed activities 92

4.3 Limit of detection (LOD) and limit of quantitation (LOQ) data 93

4.4 Instrument design 101

4.5 System components 104

4.5.1 Radiation source 104

4.5.2 Interferometer. 104

4.5.3 Two Six-device Multiplexors 104

4.5.4 Six ATR Probes. 104

4.5.5 Detector 105

4.5.6 Six Solvation Vessels 105

4.6 Instrument Control/ Acquisition/ Processing. 109

4.7 Anticipated technical difficulties. 109

4.8 Chapter summary. 109

5 Analysis of the BP Gulf oil spill by Attenuated Total Reflection-Infrared Spectroscopy and Dissolution

5.1 Research with React-IR

5.2 Experimental section 
5.2.1 Chemical and materials.

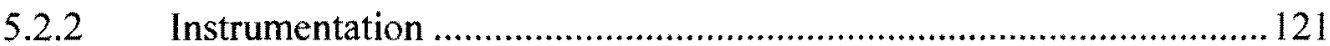

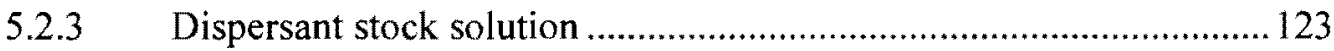

5.2.4 Crude oil sample preparation ...................................................... 123

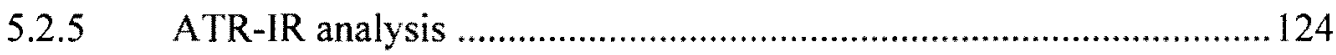

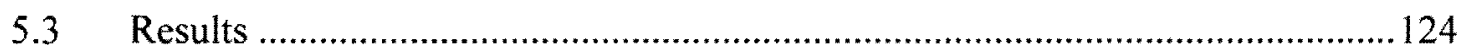

5.3.1 Standards and expected absorption values................................... 125

5.3.2 Venice oil samples dispersed using Joy soap..................................130

5.3.3 Venice oil samples dispersed using Dawn(8) soap............................133

5.3.4 Analysis by UV-Vis Spectroscopy ................................................. 137

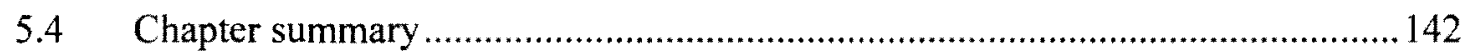

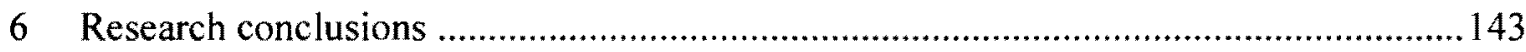

7 Appendix [A]: In situ ATR-IR Spectroscopy and Dissolution Data .............................146

8 Appendix [B]: Studying drug kinetics via ATR-IR Spectroscopy .............................. 163

9 Appendix [C]: New Instrument Design ("Solv-IR") attachments................................ 167

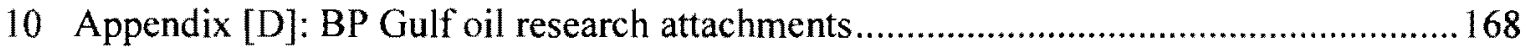




\section{List of figures}

Figure 1-1 Dissolution apparatus: Noyes and Whitney (Ref 1) (Figure

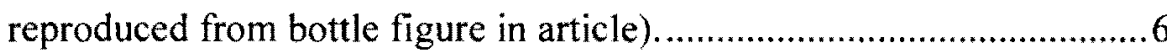

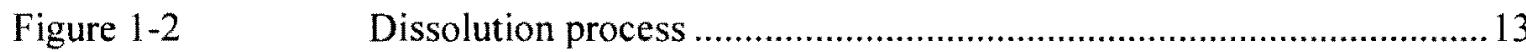

Figure 1-3 Importance of sink conditions in dissolution: dissolution of benzoic

acid at low concentrations: Underwood and Cadwallader (Ref 8)

(Figure reproduced from figure-1 in article) .................................... 16

Figure 1-4 Importance of sink conditions in dissolution: dissolution of benzoic

acid at higher concentrations: Underwood and Cadwallader (Ref 8)

(Figure reproduced from figure-3 in article)

Figure 1-5

Schematic illustration of Apparatus 1 and Apparatus 2

(Reproduced from USP General Chapter on Dissolution $<711>$ )

Figure 1-6

USP Apparatus 1 Specifications (Reproduced from USP General

Chapter on Dissolution $<711>$ )

Figure 1-7

USP Apparatus 2 specifications (Reproduced from USP General

Chapter on Dissolution $<711>$ )

Figure 1-9

FT-IR system schematic (Ref 23)

Figure 2-1

Salicylic acid spectra at different concentrations in $\mathrm{pH} 7.4$

phosphate buffered solution. 
Figure 2-2

Figure 2-3

Figure 2-4

Figure 2-5

Figure 2-6

Figure 2-7

Figure 3-1

Figure 3-2

Figure 3-3

Figure 3-4

Figure 3-5

Figure 3-6

Figure 3-7

Figure 3-8
Comparison of in situ ATR-IR and HPLC methods for dissolution

of a salicylic acid tablet.

Calibration curves of acetaminophen standards at $\mathrm{pH} 5.8$ : UV/Vis

absorbance at $296 \mathrm{~nm}$ and IR peak intensity at $1246 \mathrm{~cm}^{-1}$

Simultaneous dissolution of acetaminophen and salicylic acid tablets: in situ ATR-IR vs. HPLC

ATR-IR spectra of Excedrin $\mathbb{B}$ caplets at different time-points

during dissolution in $\mathrm{pH} 7.4$ phosphate buffered solution .55

Dissolution of acetylsalicylic acid and acetaminophen in

Excedrin $\otimes$ caplets: in situ ATR-IR vs. HPLC

Compounds studied/of interest for future work

Structures of acetylsalicylic acid (aspirin)

Synthesis of aspirin-late 1800's

Chemical structures studied .65

Ferric chloride color test- control samples

Waterfall plot of aspirin tablet dissolution

Hydrolysis of Aspirin Tablets (325 mg) in pH 1.2 SGF Solution

$(n=2)$ .76

Ferric chloride color test for salicylic acid (tablet dissolution) .76

Hydrolysis of acetylsalicylic acid powder to salicylic acid

hydrosylate in $\mathrm{pH} 1.2$ solution 
Figure 3-9

Figure 3-10

Figure 3-11

Figure 3-12

Figure 3-13

Figure 4-1

Figure 4-2

Figure 4-3

Figure 4-4

Figure 4-5

Figure 4-6

Figure 4-7

Figure 4-8

Figure 5-1

Figure 5-2
Release profile of Salicylic Acid ("SA") during hydrolysis in SGF

(pH1.2): powder vs. tablet. .81

Release profile of acetylsalicylic acid (aspirin) during hydrolysis:

powder vs. tablet 82

Release profile of acetylsalicylic acid (aspirin) and salicylic acid hydrosylate during hydrolysis: powder vs. tablet 83

Typical calculation plot of rate from dissolution 86

Rate of dissolution for Aspirin (based on slope of dissolution plot) ....86

Limit of detection of ATR-IR system 95

Fiber optic probes specifications (ref 56) .96

Calculated LOD and LOQ values

LOD/LOQ Sample Calculation Sheet- DiComp Probe Results-1 .98

LOD/LOQ Sample Calculation Sheet- Flow Cell Probe Results-1 .... 100

Diagram of proposed Solv-IR Instrument 103

Sketch of ATR crystal embedded in the side wall of the $1 \mathrm{~L}$ glass vessel. The area directly under the stir shaft is known to have poor mixing and as indicated by the term "dead zone". 106 Top plate of constant rpm stirring assembly showing motor, main gear and minor gears which drive the stir shafts in each vessel 108

Photo of oil rig similar to Deepwater Horizon.

Aerial view: location of BP Gulf oil samples 118 
Figure 5-3

Figure 5-4

Figure 5-5

Figure 5-6

Figure 5-7

Figure 5-8

Figure 5-9

Figure 5-10

Figure 5-11

Figure 5-12

Figure 5-13

Figure 5-14

Figure 5-15

Figure 5-16

Figure 7-1
Zoom view: location of BP Gulf oil samples [Both samples were

collected near the UNO site]

Ocean depth diagram showing location of BP Gulf oil samples

(reproduced and modified from Ref 71 )

Instrument photo: ATR-IR and dissolution apparatus

Gulf oil vs. blank (ocean water) standard spectra

Baseline corrected: ATR-IR scans of BP oil and Ocean Water

samples

Photo of vessels at different time points during the dispersion

experiments

ATR-IR scans of Venice oil and $50 \mathrm{~mL}$ of Joy soap.

ATR-IR scans of $50 \mathrm{~mL}$ of Joy soap only (Blank solution)

ATR-IR scans of Venice oil and $50 \mathrm{~mL}$ of Dawn $(\mathbb{R}$ soap 134

ATR-IR scans of $50 \mathrm{~mL}$ of Dawn $(\mathrm{B}$ soap only (Blank solution) 135

Visible spectra of chlorophyll a and b

Chemical structure of chlorophyll a (Chem. Draw).

Chemical structure of chlorophyll b (Chem. Draw)

UV/Vis spectra of BP oil samples in different solvents: $1 \mathrm{~mm}$ cell:

spectrophotometer fully covered from outside lighting

Linearity of salicylic acid in pH 7.4 phosphate buffer (corresponds

to Figure 2-1 in Chapter 2). 153 
Figure 7-3

Dissolution results for salicylic acid in $\mathrm{pH} 7.4$ phosphate buffer:

HPLC vs. IR data (corresponds to Figure 2-2 in Chapter 2).

Figure 7-4

ATR-IR System photo

Figure 7-5

Mettler Toledo ATR-IR/ Dissolution Systems 157

Figure 7-6

Flow Cell Fiber Optic Probe:

158

Figure 7-7

K4 Fiber Optic Probe

Figure 7-8

HPLC analysis for Salicylic Acid calibrator tablets 160

Figure 7-9

HPLC Method Parameters: Single Component and Multi

Component Experiments

Figure $7-10$

Typical Excedrin $\mathbb{B}$ experiment spectra. 162

Figure 7-11

Excedrin $\mathbb{B}$ HPLC Data 162

Figure 8-1

Salicylic Acid standard spectra 163

Figure 8-2

Acetylsalicylic Acid standard spectra 164

Figure 8-3

Rate Order Formulas and Equations (Reproduced from Wikipedia

website on Rate Order) 166

Figure 9-1

ATR-IR Technique Diagram (courtesy of Jian Wang, Ph.D.,

Mettler Toledo) 167

Figure 10-1

Detergents used during BP Gulf oil research.... 168

Figure 10-2

IR Spectra of Gulf Oil from Riser: Pre- April 20, 2010

Figure 10-3

Thermo Nicolet 4700: \% Transmittance of OIL samples 171 
Figure 10-4 


\section{List of tables}

Table 1-1

Dissolution apparatus in the pharmaceutical industry

.20

Table 1-2

Absorption bands (Ref 21) (Reproduced from table of frequencies

found on CSU Website link)

Table 2-1

Phosphate buffer preparations as per United States Pharmacopeia

Table 2-2

Method development summary for Salicylic Acid (Linearity and

Dissolution results)

Table 2-3

$\%$ Dissolved of salicylic acid: Absolute \% dissolved difference

between ATR-IR and HPLC results.

Table 2-4

Dissolution of acetylsalicylic acid (aspirin) and acetaminophen in

Excedrin $\otimes$ caplets: ATR-IR raw data

Table 2-5

Dissolution of acetylsalicylic acid (aspirin) and acetaminophen in

Excedrin $®$ caplets: HPLC raw data

Table 2-6

\% Dissolved of acetaminophen: Difference between ATR-IR and

HPLC results

Table 2-7

\% Dissolved acetylsalicylic acid (aspirin) analysis: Difference

between ATR-IR and HPLC results.

Table 3-1

Hydrochloric acid buffer preparations as per United States

Pharmacopeia (USP)

Table 3-2

Ferric chloride color test concentrations 
Table 3-3

Table 3-4

Table 4-1

Table 5-1

Table 5-2

Table 5-3

Table 5-4

Table $5-5$

Table 5-6

Table 8-1

Table 10-1
Ferric chloride color test observations [added $2 \mathrm{mg} / \mathrm{ml} \mathrm{Fe}$ (III)

solution] 73

Kinetic rates for hydrolysis of aspirin in acidic medium 85

LOD/LOQ Sample Calculation Sheet- DiComp Probe Result-2

Chemicals used in commercial dispersants (COREXIT 9500 and 9527) 116

Experimental details and sample information. 122

Expected FT-IR stretching values for crude oil and sulfonated

soaps..... 126

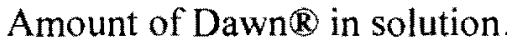
136

Amount of BP oil in solution 136

$\%$ Dispersant calculated 136 Aspirin FT-IR Frequencies of Interest. 165 Amount of Dawn $®$ dishwashing detergent added 170 


\section{Abbreviations}

ATR

ATR-IR

API

ASA

BA

BE

Disso

EPA

FDA

GI

$\mathrm{HCl}$

HPLC

HPMC

IR

LOD

LOQ

MDL

MCT

NSF

OTC

PEG

RPM

SA

SGF

TFA

USP

UV

Vis
Attenuated Total Reflection

Attenuated Total Reflection-Infrared Spectroscopy

Active Pharmaceutical Ingredient

Acetylsalicylic acid

Bioavailability

Bioequivalence

Dissolution

Environmental Protection Agency

Food and Drug Administration

Gastrointestinal

Hydrochloric Acid

High Performance Liquid Chromatography

Hydroxypropylmethylcellulose

Infrared Spectroscopy

Limit of Detection

Limit of Quantitation

Method Detection Limit

Mercury-Cadmium-Telluride

National Science Foundation

Over the Counter

Poly (ethylene glycol)

Revolutions per Minute

Salicylic acid

Simulated Gastric Fluid

Trifluoroacetic acid

United States Pharmacopeia

Ultra-Violet

Visible 


\section{$1 \quad$ Introduction and Literature Overview}

Dissolution is a critical test for measuring the performance of a pharmaceutical formulation. The overall performance of the drug pertains to the rate of release of the drug. There are many variables that affect the dissolution rate of the drug. Recently, the importance of the dissolution test has increased substantially as indicated by the high level of regulations imposed on the industry by the various health agencies around the world. Dissolution testing plays several important roles in the pharmaceutical industry. First, the test is a quality control tool that measures change in composition of the formulation. Some of the relevant changes the dissolution test is able to detect include changes caused by temperature, humidity and photosensitivity. Second, the test is also important for formulation development. During development of a drug product, the formulators use the dissolution test to distinguish between different variations of the drug product. Such variations may include characteristics of the active pharmaceutical ingredient (API) (e.g., particle size, crystal form, and bulk density), drug product composition (e.g., drug loading, and the identity, type, and levels of excipients) and the drug product manufacturing process (e.g., compression forces, equipment). In the pharmaceutical industry it is important to produce several variations of the drug product since these are needed to access the drugs' performance in clinical trials. From the clinical trials, the efficacy of the variants are distinguished and obtained. Third, once in vivo (clinical trials) data has been established for the drug product, a correlation between in vivo (human blood data)/in vitro (lab dissolution results) is made using the dissolution studies. 
Dissolution, as per the IUPAC, is defined as the mixing of two phases with the formation of one new homogeneous (i.e. the solution) phase and is pharmaceutically defined as the rate of mass transfer from a solid surface into the dissolution medium or solvent under standardized conditions of liquid/solid interface, temperature and solvent composition. ${ }^{1}$ It is a dynamic property that changes with time and explains the process by which a homogenous mixture of a solid or a liquid can be obtained in a solvent. It happens to chemically occur by the crystal break down into individual ions, atoms or molecules and their transport into the solvent. ${ }^{2}$ Dissolution is controlled by the affinity between the solid substance and the solvent. Moreover, the dissolution rate plays an important role in the understanding the chemistry of solvation. The dissolution rate is defined as the amount of drug substance that goes into solution per unit time under standardized conditions of liquid/solid interface, temperature and solvent composition.

In order for drug molecules to be transported into solution, they must detach from the solid surface and form solvated molecules. This phenomenon is known as solvation. Regardless of whether a solid is crystalline or amorphous, neighboring molecules are closely associated with each other though intermolecular forces. The dissolution process involves two main steps: the first step is the interaction between the solid and solvent molecules (solvation). The second step is the mass transport of solvated molecules to the bulk solution. Solubility controls the first step while transport controls the second. The first step of dissolution is considered a physico-chemical reaction. The solvation process is reversible, and solubility is

\footnotetext{
'IUPAC, Compendium of Chemical Terminology, 2nd ed. (the "Gold Book") (1997). Online corrected version: (2006-) "dissolution".

${ }^{2}$ Dressman, J. J.; Kramer, J. K., Eds. Pharmaceutical Dissolution Testing; Taylor and Francis: Florida, 2005.
} 
reached when the reaction reaches equilibrium. The dissolution process can also be described by the Gibbs free energy equation shown below:

\section{(Equation 1)}

$$
\Delta G=\Delta H-T \Delta S
$$

This reaction involves the breaking and formation of new intermolecular interactions. The net entropy is usually positive in dissolution since dissolution favors disorder. Moreover, the net enthalpy plays a crucial role in this equation. Dissolution is usually determined by the net enthalpy change. If the net enthalpy is less than or equal to zero, then the reaction will occur until all solid particles are dissolved. However, if the net enthalpy is positive, the reaction will occur until equilibrium is reached. In addition, the reaction rates in dissolution could be described kinetically by the following equations:

\section{(Equation 2)}

$$
V=K_{1}[\text { Drug }][\text { Solvent }]
$$

\section{(Equation 3)}

$$
K_{1}=A e^{-E a / R T}
$$

Where $\mathrm{V}$ is the reaction rate, Ea is the activation energy, $\mathrm{R}$ is a constant and $\mathrm{T}$ is temperature. The rate of dissolution is governed by the slower step of the twp step process mentioned earlier. Therefore, at room temperature, the rate of solvation is so fast that equilibrium is 
typically instantaneous. The mass transport step is usually much slower and becomes the rate limiting step in the dissolution process. ${ }^{3}$

Next, this chapter of the thesis will focus the describing the history of dissolution testing which has led to many types of pharmaceutical dosage forms as well as different types of apparatus systems. Even though there is increased interest in this area, the techniques used for studying dissolution rates remain fairly constant. In fact, there are only a handful of instruments used to analyze and understand the dissolution rates of drugs. This chapter will also discuss the current techniques used for dissolution testing.

Last but not least, a thorough literature search into the use of ATR-IR spectroscopy as a dissolution technique was made and will be discussed in this chapter. The research has found that FTIR imaging and NIR spectroscopy have been used to study dissolution testing. Although FTIR imaging utilizes the same spectroscopic region as the proposed research, it is more of qualitative test than a quantitative one. Furthermore, NIR was used to study dissolving reactions. The major weakness of the NIR region is that the absorption bands occurring there are the overtones of the fundamental bands residing in the mid-IR region. As a result, they are relatively weak and not clearly delineated. This makes quantitative calculations complex and calibration procedures quite laborious and not transferable from one instrument to another. This research focuses on the mid-IR region. The mid-IR spectral region is where most of the fundamental structural information is produced. Also, because molecules differ from each other by having different combinations of functional groups, their mid-IR spectra can be used to identify them and characterize their structure.

\footnotetext{
${ }^{3}$ Hanson, R; Gray, V. Handbook of Dissolution Testing, 3rd ed., Dissolution Technologies: Delaware, 2004.
} 


\section{$1.1 \quad$ History}

The earliest theories of the dissolution were based on physicochemical properties. The earliest reference to dissolution dates back to 1897 when Noyes and Whitney published an article entitled, "The Rate of Solution of Solid Substances in Their Own Solution." ${ }^{4}$ Noyes and Whitney investigated the phenomenon of dissolution by using two slightly soluble substances; benzoic acid and lead chloride. They developed a rotating bottle technique coupled with a titration method to determine the dissolution rates of these two active drugs. Refer to Figure 1-1 for a schematic diagram of the first dissolution apparatus.

\footnotetext{
${ }^{4}$ Noyes, Arthur A.; Whitney, Willis R. The rate of solution of solid substances in their own solutions. Journal of the American Chemical Society 1897, 19(12), 930-934.
} 
Figure 1-1 Dissolution apparatus: Noyes and Whitney (Ref 1) (Figure reproduced from bottle figure in article).

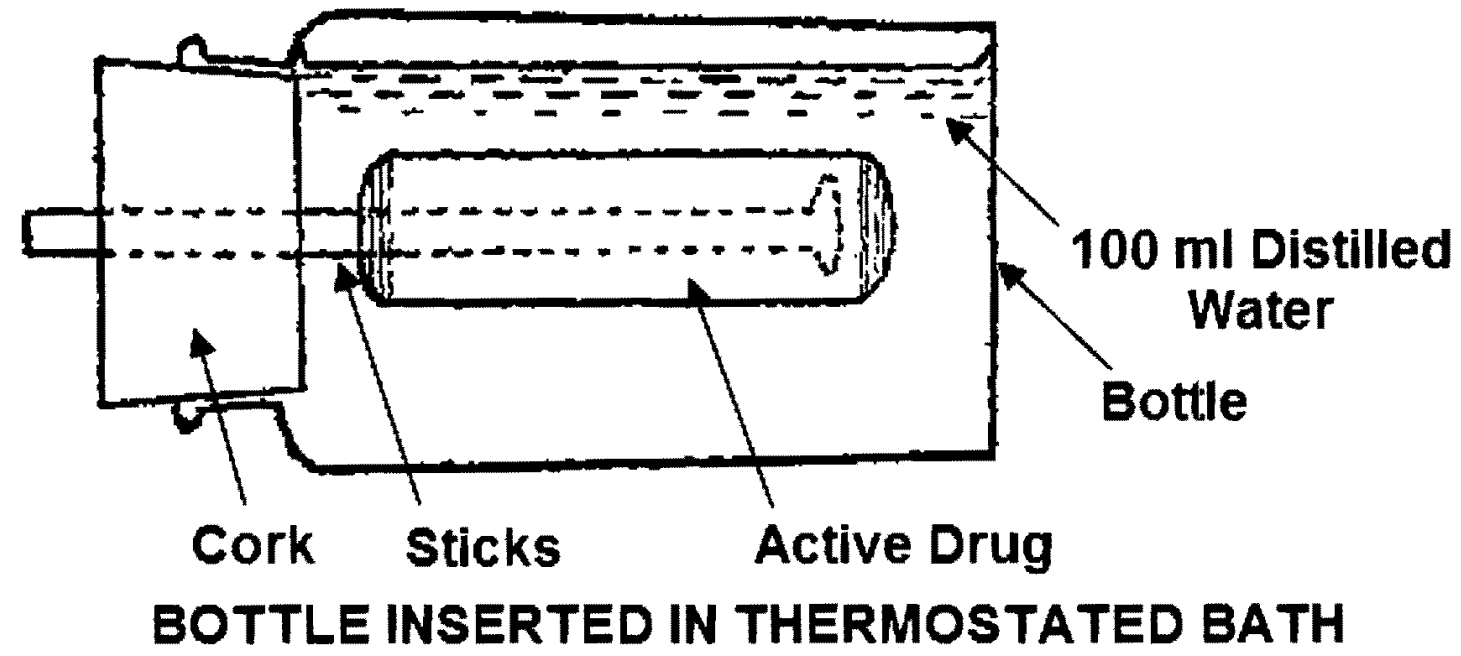


Noyes and Whitney determined that the velocity of the solution is proportional to the difference between the concentration of the saturated solution and that of the solution present at the moment in question. They determined the following dissolution rate equation as shown in the equation 4 below:

\section{(Equation 4)}

$$
\frac{d x}{d t}=C(S-x)
$$

where $\mathrm{S}$ represents the solubility of the substance $(\mathrm{mg} / \mathrm{mL}), \mathrm{x}$ the concentration (usually $\mathrm{mg}$ or mmol) at the expiration of the time $\mathrm{t}$ (seconds) and $\mathrm{C}$ is a constant. Integration of the equation above gives equation 5 :

\section{(Equation 5)}

$$
C=\frac{1}{T} \log _{e} \frac{S}{(S-x)}
$$

In order to obtain values for $C$ in the equation, the solubility or $S$ of the substance must be known. In addition, Noyes and Whitney found that the dissolution rate is controlled by a thin layer of saturated solution that forms instantly around a solid particle. Also, the rate at which a solid substance dissolves in its own solution is proportional to the difference between the concentration of the solution and the concentration of the saturated solution.

In 1904, Nernst and Brunner modified the Noyes-Whitney equation by applying Fick's law of diffusion. ${ }^{5}$ They were able to show a relationship between the dissolution rate constant and

\footnotetext{
${ }^{5}$ Nernst, W ; Brunner, E. Velocity of Reaction in Non-Homogeneous System. Physical Chemistry 1904, 47, 56-
} 102. 
the diffusion coefficient. Refer to equation 6. The equation for Fick's law of diffusion is shown below:

(Equation 6)

$$
k=\frac{D S}{V h}
$$

where D represents the diffusion coefficient $\left(\mathrm{cm}^{2} / \mathrm{s}\right), \mathrm{S}$ is surface area $\left(\mathrm{cm}^{2}\right)$ of the diffusion layer, $\mathrm{V}$ the solution volume $(\mathrm{mL})$ and $\mathrm{h}$ is the diffusion layer thickness $(\mu \mathrm{m})$. By applying Fick's law, equation 7 was derived as shown below where $\mathrm{k}$ is the intrinsic dissolution rate constant:

(Equation 7)

$$
\frac{d x}{d t}=k\left(\frac{D S}{V h}\right)(C s-C t)
$$

Based on the equations discussed earlier, it is evident that certain properties of the drug are important when determining dissolution. These properties include the solubility, physical form, media and $\mathrm{pH}$ of the media.

In the 1950's the emphasis shifted from studying the effects of physicochemical properties of drugs on dissolution to correlation of dissolution to bioavailability (BA) of dosage forms. Oral bioavailability is a key pre-requisite for any orally administered drug to be systemically effective. The United States Food and Drug Administration (FDA) defines bioavailability according to 21 CFR $320(\S 320.1)^{6}$ as: the rate and extent to which the active ingredient or active moiety is absorbed from a drug product and becomes available at the site of action. For drug products that are not intended to be absorbed into the bloodstream, bioavailability

\footnotetext{
${ }^{6}$ Bioavailability and Bioequivalence Requirements. Code of Federal Regulations, Part 320, Title 21, 2003.
} 
may be assessed by measurements intended to reflect the rate and extent to which the active ingredient or active moiety becomes available at the site of action. This definition focuses on the processes by which the active ingredients or moieties are released from an oral dosage form and move to the site of action. ${ }^{7}$ The definition above focuses on the process by which the active drugs or ingredients are released from an oral dosage form and move to the site of action in the human body. As noted in the guidance document, bioavailability is documented by developing a systemic exposure profile. This profile can be achieved by measuring the concentration of active ingredients and its active metabolites over time in samples collected from the systemic circulation. Systemic circulation describes the part of the cardiovascular system within the human body which carries oxygenated blood away from the heart and returns deoxygenated blood back to the heart. ${ }^{8}$ Bioequivalence (BE) also plays an important role when considering dissolution. As per the FDA, bioequivalence is defined in $\S 320.1$ as: the absence of a significant difference in the rate and extent to which the active ingredient or active moiety in pharmaceutical equivalents or pharmaceutical alternatives becomes available at the site of drug action when administered at the same molar dose under similar conditions in an appropriately designed study. Both bioequivalence and bioavailability focus on the release of a drug substance from a drug product and subsequent absorption in the system circulation. Moreover, this is the basis for pharmacokinetics.

Pharmacokinetics may be simply defined as what the body does to the drug. It attempts to study the mechanisms of absorption and distribution of the administered drug, the rate at

\footnotetext{
${ }^{7}$ Guidance for Industry: Bioavailability and Bioequivalence Studies for Orally Administered Drug ProductsGeneral Considerations 2003. U.S. Department of Health and Human Services, FDA [Online], http:/www.fda.gov/cder/guidance/index.htm (accessed May 1, 2011).
} 
which the drug begins and duration of the effect. Bioavailability and bioequivalence rely on pharmacokinetics measurements such as AUC and Cmax that reflect the systemic exposure. AUC is the area under the plasma/serum/blood concentration-time curve from time zero to time $t$, where $t$ is the last time point with measurable concentration for individual formulation. Cmax measures the peak exposure by measuring the peak drug concentration obtained directly from the data without interpolation. ${ }^{9}$

In the 1950 's, the scientific community focused its interests on dissolution as the rate process for controlling the absorption of active drugs into the bloodstream. In 1951 L.J. Edwards suggested that the analgesic activity of aspirin tablets can be manipulated by its rate of dissolution within the GI tract. ${ }^{10}$ Edwards studied the kinetics of the dissolution of aspirin in water at different $\mathrm{pH}$ 's but over the same temperature range. The solubilities of aspirin were measured and diffusion rates were recorded at several concentrations. He determined that the diffusion coefficients are not independent of the concentration. Edwards suggested that the dissolution of an aspirin tablet in the stomach and intestine is the rate process controlling the absorption of the aspirin into the bloodstream.

In 1960, Levy and Hayes used a beaker and a three blade stirrer to demonstrate that the incidence of GI irritation of different brands of aspirin is dependent on the dissolution rates of

\footnotetext{
${ }^{8}$ Wilding, I. Bioequivalence testing for locally acting gastrointestinal products: what role for gamma scintigraphy? Journal of Clinical Pharmacology 2002, 42(11), 1200-1210.

${ }^{9}$ Guidance for Industry: Bioavailability and Bioequivalence Studies for Orally Administered Drug ProductsGeneral Considerations 2003. U.S. Department of Health and Human Services, FDA [Online], http://www.fda.gov/cder/guidance/index.htm (accessed May 1, 2011).

${ }^{10}$ Edwards, L. J. Solution and diffusion of aspirin in aqueous media. Transactions of the Faraday Society 1951, $47,1191-1210$.
} 
the dosage forms. ${ }^{11}$ In other words, the dissolution rates were critical for the release of aspirin into the body and causing irritation. Thus, if the release profile is controlled then the irritation levels are controlled. During this period it was recognized that although disintegration was a critical process, de-aggregation was also essential for bioavailability. Disintegration is the process by which an object breaks down or loses it cohesive ability. De-aggregation ${ }^{12}$ is the dispersion of particles and dislodgment of the granules. Usually, surfactant in solution helps in the de-aggregation process. Figure $1-2$ is an illustration of where disintegration and deaggregation occur in the dissolution process. The dissolution process occurs in three phases. In Phase I, the rate of dissolution is very slow as during this process solvent diffuses into the solid material (e.g., the pill) via a process called wetting. The initial period is called "mechanical lag". Also, during this phase the solid particles undergo disintegration and deaggregation. In Phase II, the solid particles are well dispersed into the solvent and the rate of dissolution is more rapid. This rate follows a zero order kinetics profile (rate $=\mathrm{dx} / \mathrm{dt}=\mathrm{k}$ ). This is followed by Phase III in which $1-2 \%$ of the active ingredient is dissolved at a much slower rate. The slower rate is caused by particles that have not been properly wetted and have not been de-aggregated. In this simulated diagram, approximately $5 \%$ of the active ingredient does not dissolve. While, this level of incomplete dissolution is unlikely to occur in a well formulated pill, this section of the diagram nicely represents a process by which some of the active ingredient never dissolves. Two primary factors preventing complete dissolution are occlusion and saturation. In occlusion, the active ingredient is so well aggregated that it is

\footnotetext{
${ }^{11}$ Levy, G.; Hayes, B. A., Physiochemical basis of the buffered acetylsalicylic acid controversy, New England Journal of Medicine, 1960, 21, 1053-1058.

${ }^{12}$ Liu, J.; Stewart, P. J. Deaggregation during the dissolution of benzodiazepines in interactive mixtures. Journal of Pharmaceutical Sciences 1998, 87(12), 1632-1638.
} 
poorly wetted that dissolution is not accomplished during the time of the experiment. Alternatively, saturation may have occurred whereby the active ingredient has reached its thermodynamic limit of solubility which prevents complete dissolution. Equilibrium suggests that complete dissolution is difficult to achieve. Equilibrium is the condition of the system in which competing influences are balanced. 
Figure 1-2 Dissolution process

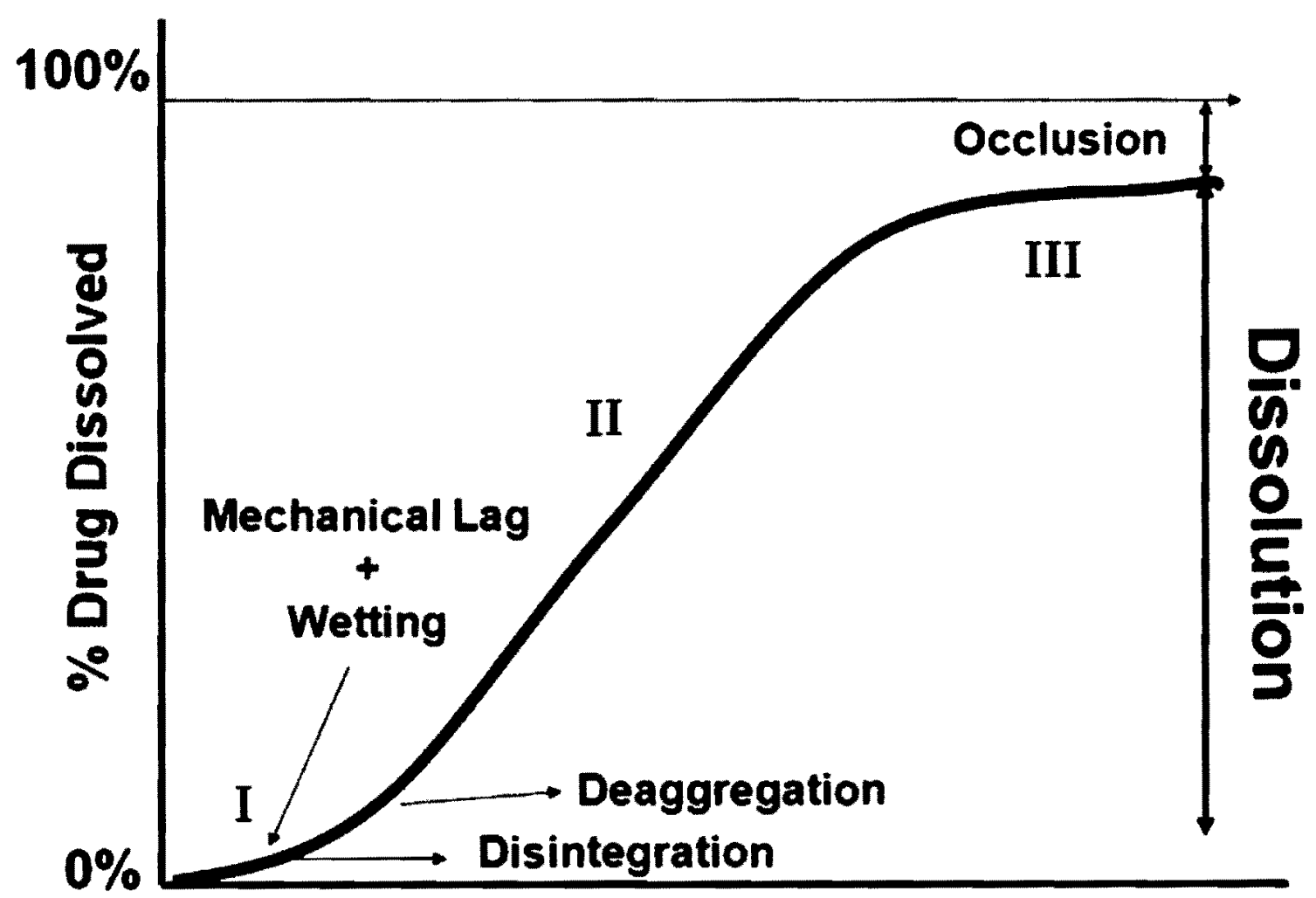

Time 
In 1978, Underwood and Cadwallader ${ }^{13}$ modified the Noyes-Whitney equation to take into consideration sink conditions. They showed that solubility plays an important role in dissolution. Sink conditions exist when the volume of the dissolution medium is three to ten times larger than the volume required to make a saturated solution of the solute. Thus, sink conditions exist when the concentration $(C)$ of active drug in the medium at time $t$ is much smaller than the saturation concentration (Cs). They revised the Noyes Whitney equation accordingly. " $\mathrm{M}$ " represents the mass of substance remaining to be dissolved, " $\mathrm{A}$ " represents the surface area of the drug exposed to the dissolution medium and " $k$ " represents the intrinsic dissolution rate constant (dissolution constant).

\section{(Equation 8)}

$$
\frac{d M}{d t}=-k A(C s-C)
$$

Moreover, as the concentration of the solution increases, the equation predicts that $\mathrm{dM} / \mathrm{dt}$ decreases. When the concentration of the saturated solution is much larger than the concentration of the drug $(\mathrm{Cs}>>\mathrm{C})$ in the solvent at time $\mathrm{t}$, then equation 9 is obtained.

\section{(Equation 9)}

$$
\frac{d M}{d t}=-k A C s
$$

Underwood and Cadwallader concluded that the overall dissolution rate increases with increasing dissolution rate constant $(\mathrm{k})$, increased surface area (A) and increased solubility

\footnotetext{
${ }^{13}$ Underwood, F. L.; Cadwallader, D. E. Automated potentiometric procedure for studying dissolution kinetics of acidic drugs under sink conditions, Journal of Pharmaceutical Sciences 1978, 67(8), 1163-1167.
} 
(Cs). Therefore, by maintaining $\mathrm{A}$ and $\mathrm{Cs}$ as constants, the dissolution rate constant can be conveniently measured.

Furthermore, they stated that the dissolution rate of a drug from a constant surface area under sink conditions should follow zero-order kinetics. For zero-order kinetics, a linear plot of [drug concentration] vs. time can be used to determine the rate $(\mathrm{k})$. This value can be interpolated from the slope. However, when there is a much higher level of a solute such as of benzoic acid in solution, the dissolution rate decreased slightly as shown in Figure 1-3 and Figure 1-4 below. Underwood and Cadwallader document that a possible reason for this decrease is that the concentration of the buffering compounds in the dissolution medium could retard the dissolution of the solute, i.e., benzoic acid. As the concentration of benzoic acid in solution increases, there is competition for solvent. Also, the buffer $\mathrm{pH}$ decreased slightly as the benzoic acid dissolves, which could have decreased the $\mathrm{pH}$ of the diffusion layer adjacent to the solid benzoic acid particles and caused a decrease in the dissolution rate. 
Figure 1-3 Importance of sink conditions in dissolution: dissolution of benzoic acid at low concentrations: Underwood and Cadwallader (Ref 8) (Figure reproduced from figure-1 in article)

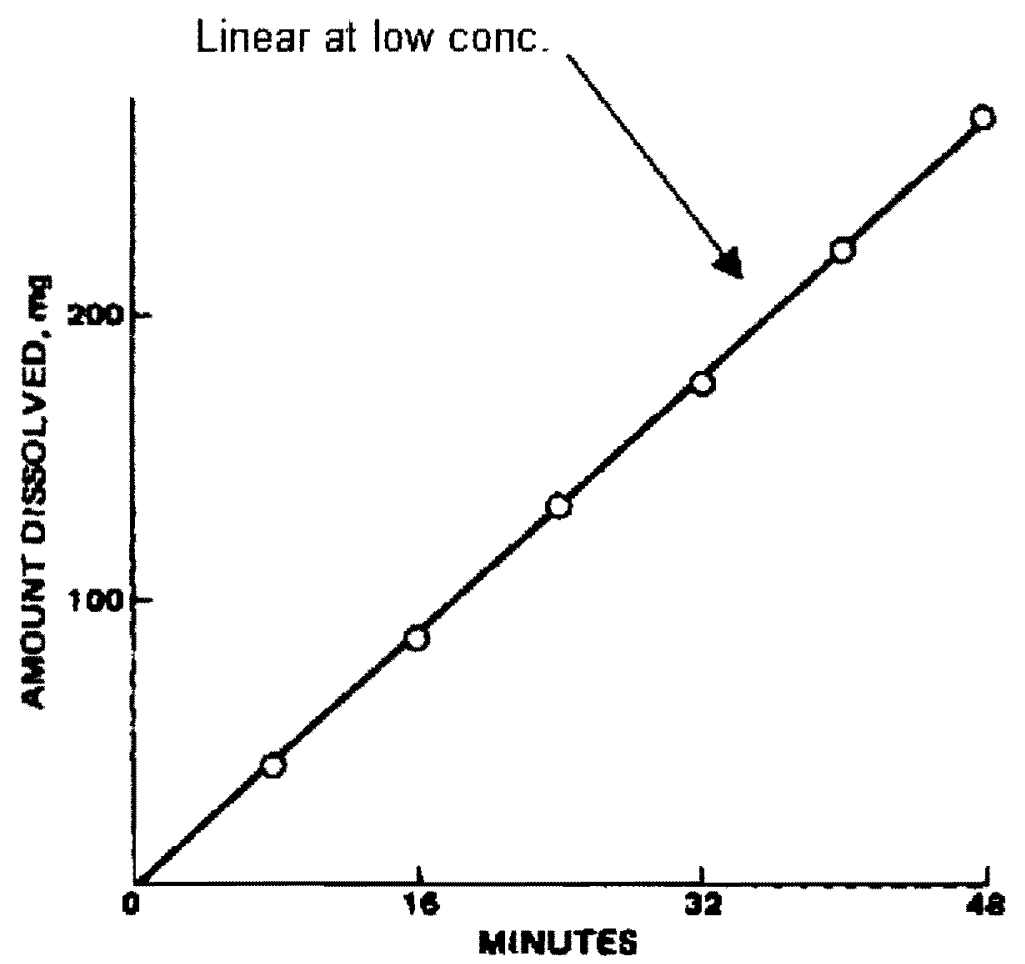

-Distolution of benzoic acid from a nondisintegratine disk in distilled water (pH-stat 6.2) at $37^{\circ}$ and $100 \mathrm{rpm}$. 
Figure 1-4 Importance of sink conditions in dissolution: dissolution of benzoic acid at higher concentrations: Underwood and Cadwallader (Ref 8)

(Figure reproduced from figure-3 in article)

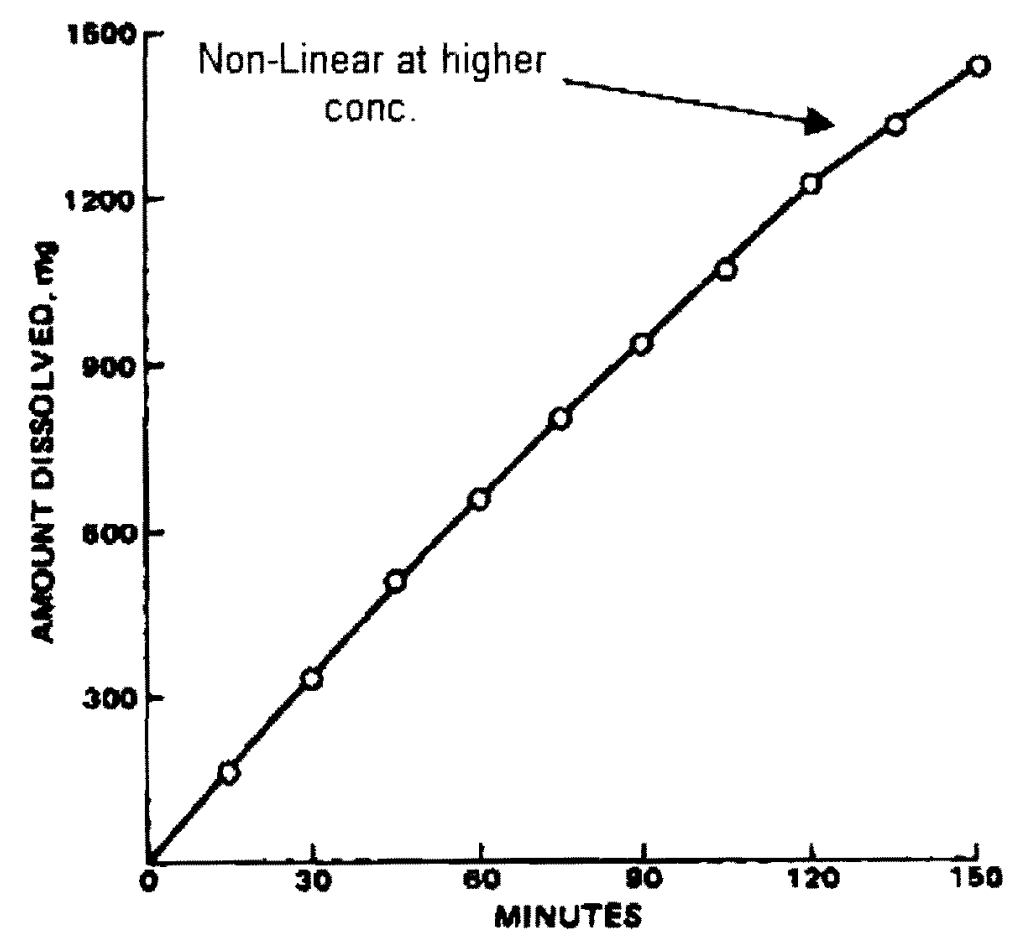

- Disualution of benzoic acid from a nondisinternating disk in buffer (pH 6.2) at $37^{\circ}$ and $100 \mathrm{rpm}$. 
As a consequence of the research that had occurred from late 1800 's to mid 1900's, in 1970 the first official dissolution test appeared in USP XVIII. The United States Pharmacopeia or $\mathrm{USP}^{14}$ is a non-governmental, official public standards-setting authority for prescription and over-the-counter medicines and other healthcare products manufactured or sold in the United States. USP also sets widely recognized standards for food ingredients and dietary supplements. They set standards for the quality, purity, strength, and consistency of these products which are critical to the public health.

Increased interest in dissolution regulations continue to grow well into the 1970's. In fact, in 1978 the FDA published the document entitled, "Guidelines for Dissolution Testing." intention behind this publication was to combine and streamline the systems and processes of different laboratories. This was due to the fact that dissolution results were observed to have high variability and minor changes in the equipment parameters increased variability. The FDA realized that they needed more controls on the tolerances of the dissolution equipment so that results are more reproducible. Additionally, the FDA and USP introduced the idea of calibrator tablets. In 1978 the USP launched three calibrator tablets: Prednisone, Salicylic Acid and Nitrofurantoin. These calibrator tablets were used during the calibration of the instrument to validate that the dissolution bath is working as indicated. The calibrator tablets have known specification limits and the calibrations of the instruments have to be within

\footnotetext{
${ }^{14}$ United States Pharmacopeia, Home Page. http://www.usp.org/ (accessed May 1, 2011)

${ }^{15}$ Guidance for Industry: Dissolution Testing of Immediate Release Solid Oral Dosage Forms 1997. U.S. Department of Health and Human Services, FDA [Online], http://www.fda.gov/cder/guidance/index.htm (accessed May 1, 2011).
} 
those limits. To gain an appreciation of the complexity of the dissolution system and equipment parameters, an overview of the technology will be given in the next section.

In 1995, the USP assigned unique numbers to the different dissolution apparatus that were available to the scientific community. Specifically, there are seven different types of dissolution equipment generally used. These are listed below in Table $1-1$. 
Table 1-1 Dissolution apparatus in the pharmaceutical industry

\begin{tabular}{|l|l|}
\hline Dissolution Apparatus Number & Dissolution Apparatus Name \\
\hline Apparatus 1 & Basket \\
\hline Apparatus 2 & Paddle \\
\hline Apparatus 3 & Reciprocating Cylinder \\
\hline Apparatus 4 & Flow Through Cell \\
\hline Apparatus 5 & Paddle over Disk \\
\hline Apparatus 6 & Cylinder \\
\hline Apparatus 7 & Reciprocating Disk \\
\hline
\end{tabular}


Apparatus 1 and 2 are most widely used in the pharmaceutical industry. As shown above the first instrument uses baskets method while the second one uses paddles. Figure $1-5$ is a schematic illustration of these two apparatuses. The apparatuses are comprised of a covered vessel, a metallic drive shaft, a motor to spin the shaft, a cylindrical basket (Apparatus 1) or a paddle (Apparatus 2), and a water bath or heated jacket capable of maintaining the temperature of the vessels at $37^{\circ} \pm 0.5^{\circ} \mathrm{C}$. 
Figure 1.5 Schematic illustration of Apparatus 1 and Apparatus 2 (Reproduced from USP General Chapter on Dissolution <711>)

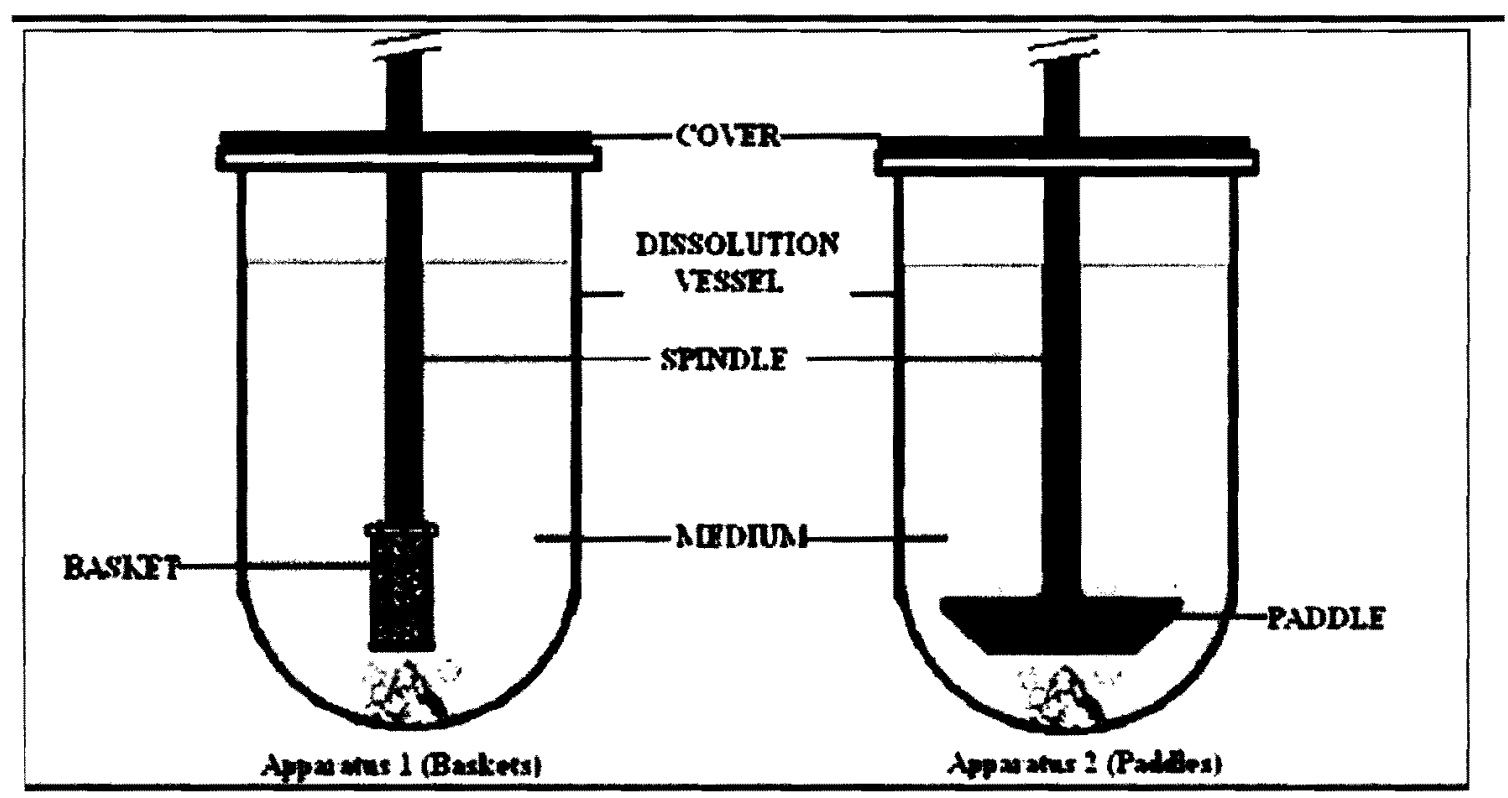


Although the figures above appear simple in design, there are strict regulations for the specifications of each component of the apparatus and tolerances for each component are specified by USP $<711>.{ }^{16}$ Refer to Figure $1-6$ and Figure $1-7$ for schematic diagrams of the specifications set by the USP for Apparatus 1 and Apparatus 2, respectively.

As a result of these regulations of the past fifty years, the number of USP monographs including dissolution monographs has exponentially increased. As shown in Figure 1-8, in 1970 , there were only twelve monographs. In May of 2011 , there were 740 dissolution USP monographs. ${ }^{171819}$

\footnotetext{
${ }^{16}$ US Pharmacopoeia \& National Formulary- USP General Chapter $<711>$ : Dissolution, USP [Online], http://www.usp.org/ (accessed May 1, 2011).

${ }^{17}$ Cohen, J; Hubert, B; Leeson, L; Rhodes, C; Robinson, J; Roseman, T; Shefter, E. The Devlopment of USP Dissolution and Drug Release Standards. Pharmaceutical Research 1990, 7(10), 983-987.

${ }^{18}$ Dokoumetzidis, A; Macheras, P. A century of dissolution research: From Noyes and Whitney to the Biopharmaceutics Classification System. International Journal of Pharmaceutics 2006, 321, 1-11.

${ }^{19}$ US Pharmacopoeia \& National Formulary- USP Monographs including dissolution (2011 summary), http://www.usp.org/ (accessed May 1, 2011)
} 
Figure 1-6 USP Apparatus 1 Specifications (Reproduced from USP General Chapter on Dissolution $<711>$ )

E

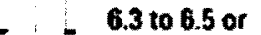

9.4 to $10.1 \mathrm{~mm}$

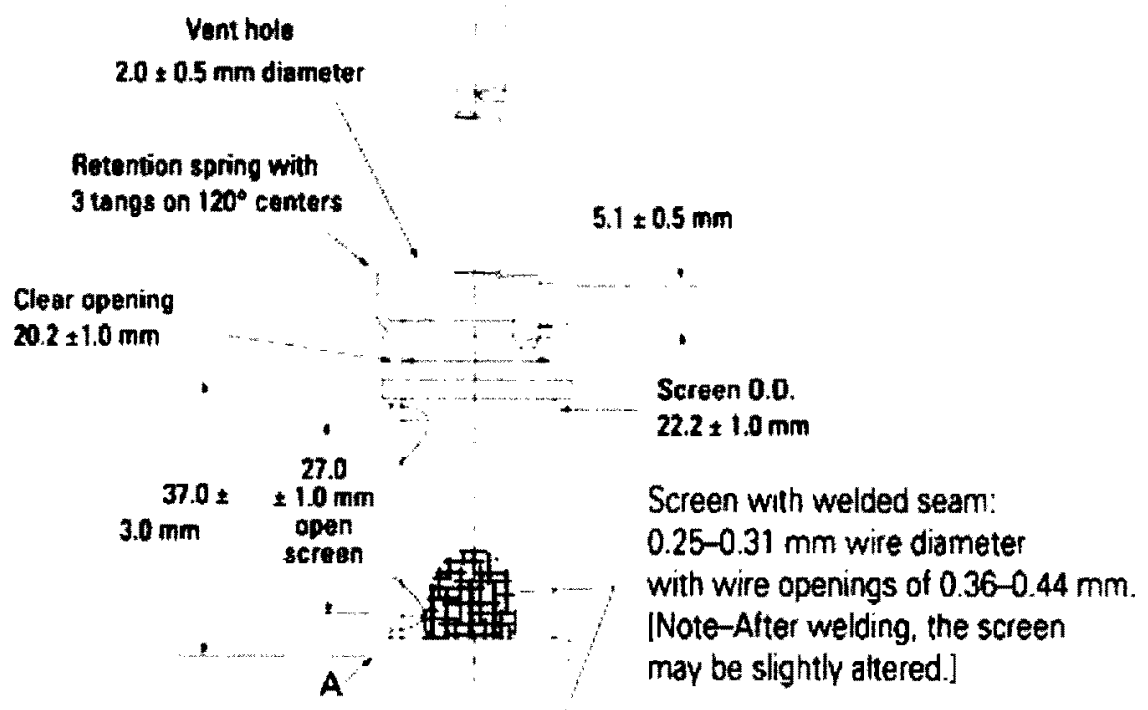

Note-Maximum allowable runout at " $A$ "

is $\pm 1.0 \mathrm{~mm}$ when the part is rotated on

center line axis with basket mounted.

$20.2+1.0 \mathrm{~mm}$

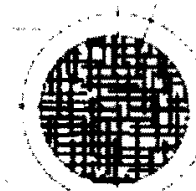

$25.0 \times 3.0 \mathrm{~mm}$ 
Figure 1-7 USP Apparatus 2 specifications (Reproduced from USP General Chapter on Dissolution $<711>$ )

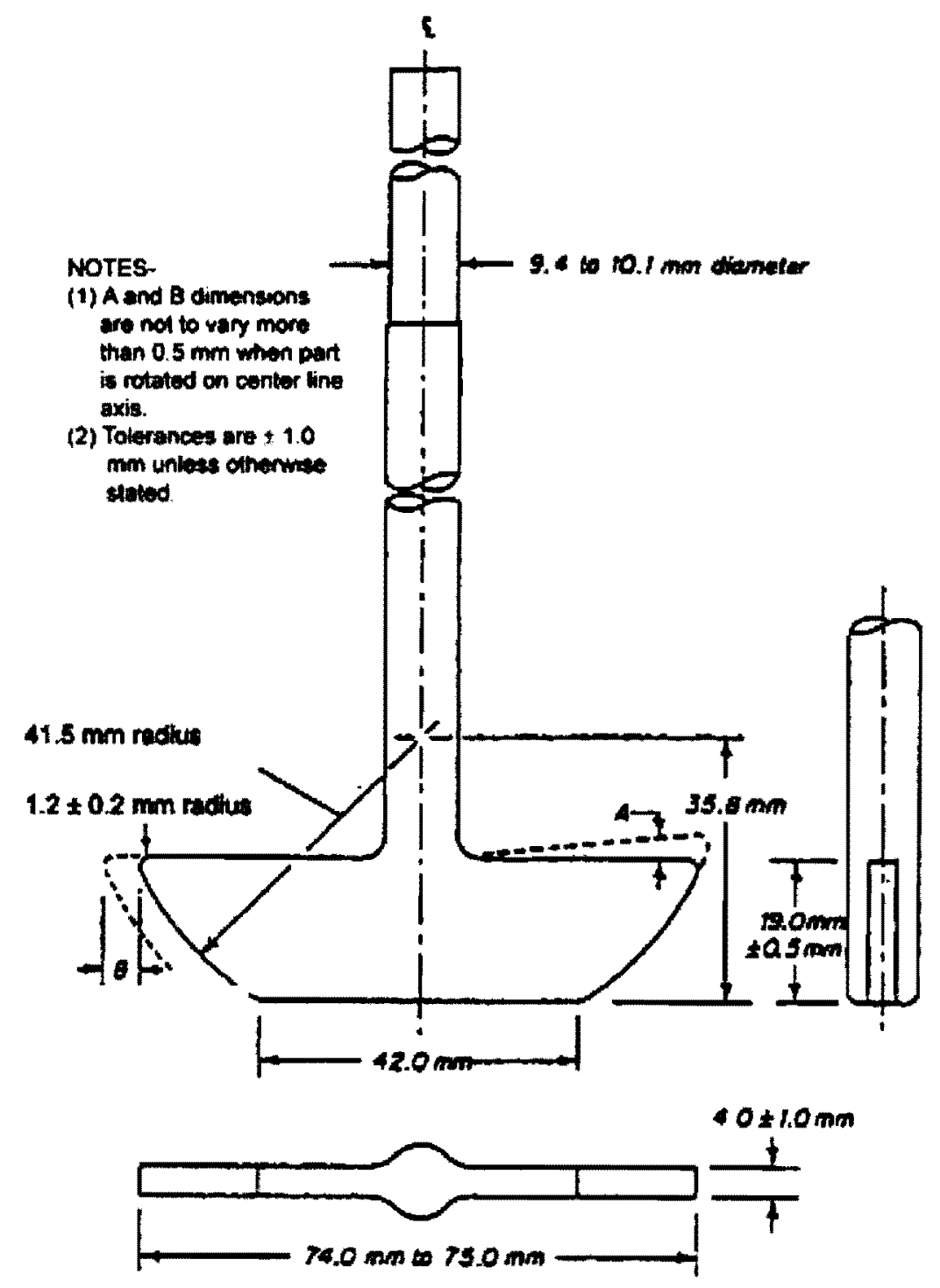


Seton Hall University

Figure 1-8 Monographs including dissolution in the United States Pharmacopeia (USP)

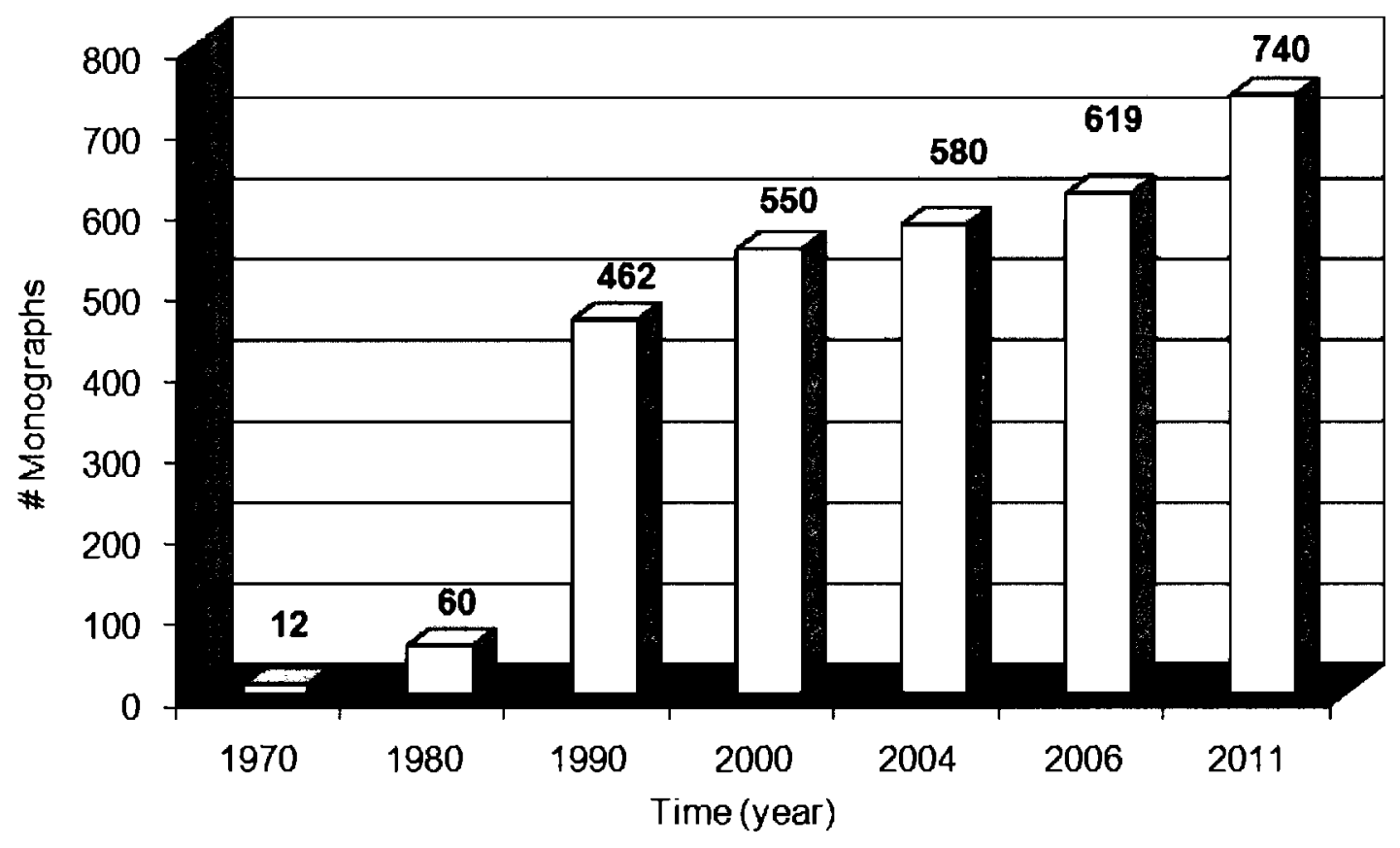


As a result of the high regulations and increased number of USP monographs including dissolution, this research will improve dissolution testing. Conventional ways of testing have been used for many years by the industry. Specifically, the UV/Vis and HPLC systems have gone through several stages of evolution but continue to be used as the standards. This research and technique will attempt to improve dissolution testing in several ways. First, this technique will allow for in-situ analysis which is very important when dealing with sensitive drug formulations. These sensitive formulations are susceptible to more sampling error when samples are withdrawn from the dissolution vessel. Second, FTIR spectroscopy can be advantageous to dissolution testing. Unlike UV/Vis spectroscopy which gives a single peak per active drug, FTIR spectroscopy can be sued to monitor drug release via monitoring of numerous functional groups. Moreover, FTIR spectroscopy can be used to track chemical transformations within the vessel. This will be discussed in chapter three of this thesis. Lastly, FTIR can be used to study multi-component drug formulations. 


\subsection{Infrared Spectroscopy}

A brief overview is warranted. Infrared spectroscopy is the spectroscopy that deals with the infrared region, $14000 \mathrm{~cm}^{-1}(714 \mathrm{~nm})$ to $10 \mathrm{~cm}^{-1}\left(1 \times 10^{6} \mathrm{~nm}\right)$, of the electromagnetic spectrum. Moreover, the IR portion of the electromagnetic spectrum is divided into three regions; nearinfrared, mid-infrared and far-infrared. The near-infrared ${ }^{20}$ energy, approximately in the region between $14000-4000 \mathrm{~cm}^{-1}$, can excite overtone or harmonic vibrations. The midinfrared ${ }^{21}$ energy, approximately in the region between $4000 \mathrm{~cm}^{-1}(2500 \mathrm{~nm})$ to $400 \mathrm{~cm}^{-1}$ $(25000 \mathrm{~nm})$, can be used to study the fundamental vibrations of structures. The far-infrared region, approximately in the region between $400-10 \mathrm{~cm}^{-1}$, can be used to study to rotations of structures. With IR spectroscopy, different functional groups adsorb at different IR bands or regions as shown in Table 1-2.2 Thus, this technique can help identify and even quantify organic and inorganic molecules.

\footnotetext{
${ }^{20} \mathrm{~J}$. Workman Jr. Interpretive spectroscopy for near-infrared, The Handbook of Organic Compounds; Academic Press: California, 2001; pp 143-182.

${ }^{21}$ Heise, H. M.; Küpper, L.; Butvina, L. N. Attenuated total reflection mid-infrared spectroscopy for clinical chemistry applications using silver halide fibers. Sensors and Actuators B: Chemical1998, 51(1-3), 84-91. ${ }_{22}$ Images and frequencies obtained from: http:/wwwchem.csustan.edu/tutorials/infrared.htm. (accessed May 1 , 2011)
} 
Table 1-2

Absorption bands (Ref 22) (Reproduced from table of frequencies found on CSU Website link)

\begin{tabular}{|c|c|c|}
\hline Bond & Compound Type & Frequency range, $\mathrm{cm}^{-1}$ \\
\hline \multirow{3}{*}{$\mathrm{C} \cdot \mathrm{H}$} & \multirow{2}{*}{ Alkanes } & 2960-2850(s) stretch \\
\hline & & $1470-1350(v)$ scissoring and bending \\
\hline & CH3 Umbrella Deformation & $1380(m-w)$ - Doublet - isopropyl t-butyl \\
\hline \multirow{2}{*}{$\mathrm{CH}$} & \multirow{2}{*}{ Alkenes } & $3080-3020(\mathrm{~m})$ stretch \\
\hline & & $1000-675(\mathrm{~s})$ bend \\
\hline \multirow{3}{*}{$\mathbf{C H}$} & Aromatic Rings & $3100-3000(\mathrm{~m})$ stretch \\
\hline & PhenyI Ring Substitution Bands & $870.675(s)$ bend \\
\hline & Phenyl Ring Substitution Overtones & 2000-1600(w) - fingerprint region \\
\hline \multirow{2}{*}{ C.H } & \multirow{2}{*}{ Akynes } & $3333-3267(\mathrm{~s})$ stretch \\
\hline & & $700.610(b)$ bend \\
\hline $\mathbf{C}=\mathrm{C}$ & Akenes & $1680-1640(\mathrm{~m}, \mathrm{w})$ stretch \\
\hline $\mathrm{C} \equiv \mathrm{C}$ & Alkynes & $2260-2100(w . s h)$ stretch \\
\hline $\mathrm{C}=\mathrm{C}$ & Aromatic Rings & $1600.1500(w)$ stretch \\
\hline $\mathrm{C} \cdot \mathrm{O}$ & Alcohols, Ethers. Carboxylic acids, Esters & $1260-1000(s)$ stretch \\
\hline $\mathrm{C}=\mathrm{O}$ & Aldenydes Ketones. Carboxylic acids. Esters & $1760-1670($ s) stretch \\
\hline \multirow{3}{*}{$\mathbf{O} \cdot \mathrm{H}$} & Monomeric -- Alcohols, Phenols & $3640-3160($ s. br) stretch \\
\hline & Hydrogen-bonded - Alcohols. Phenols & $3600-3200(b)$ stretch \\
\hline & Carboxylic acids & $3000-2500(b)$ stretch \\
\hline \multirow{2}{*}{$\mathbf{N}+\mathbf{H}$} & \multirow{2}{*}{ Arnines } & $3500-3300(\mathrm{~m})$ stretch \\
\hline & & $1650-1580(\mathrm{~m})$ bend \\
\hline $\mathrm{C}-\mathrm{N}$ & Amines & $1340-1020(\mathrm{~m})$ stretch \\
\hline $\mathbf{C} \equiv \mathbf{N}$ & Nitriles & $2260-2220(v)$ stretch \\
\hline \multirow{2}{*}{$\mathbf{N O}_{2}$} & \multirow{2}{*}{ Ntro Cornpounds } & 1660-1500(s) asymmetrical stretch \\
\hline & & 1390-1260(s) symmetric al stretch \\
\hline
\end{tabular}


The infrared spectrum of a sample can be obtained by passing a beam of infrared light through the sample. A Fourier transform instrument ${ }^{23}$ can be used to measure how much energy was absorbed by the sample over the entire wavelength range. The interferometer is a fundamentally different piece of equipment than a monochromater. The light passes through a beamsplitter, which sends the light in two directions at right angles. One beam goes to a stationary mirror then back to the beamsplitter. The other goes to a moving mirror. The motion of the mirror makes the total path length variable versus that taken by the stationarymirror beam. When the two meet up again at the beamsplitter, they recombine, but the difference in path lengths creates constructive and destructive interference: an interferogram: A mathematical function called a Fourier transform converts an intensity-vs.-time spectrum into an intensity-vs.-frequency spectrum. Refer to equation $10 . \mathrm{A}(\mathrm{r})$ and $\mathrm{X}(\mathrm{k})$ are the frequency domain and time domain points, respectively, for a spectrum of $\mathrm{N}$ points.

\section{(Equation 10)}

$$
A(r)=\sum X(k) \exp \left(-2 \pi \frac{i r k}{N}\right)
$$

The FT-IR system can produce both transmittance and absorbance spectrum. Refer to Figure 1-9 for an illustration of the FT-IR system. ${ }^{24}$ The interferogram represents the light output as a function of mirror position. The FT-IR raw data is processed to give the actual spectrum of light output as a function of wavenumber.

\footnotetext{
${ }^{23}$ Smith, B.C. Fundamentals of Fourier Transform Infrared Spectroscopy; CRC Press: London, 1996; Chapter 4. ${ }^{24}$ Image obtained from http//mmrc.caltech.edu/FTIR/FTIRintro.pdf (accessed July 1, 2011).
} 
Figure 1-9 FT-IR system schematic (Ref 24)

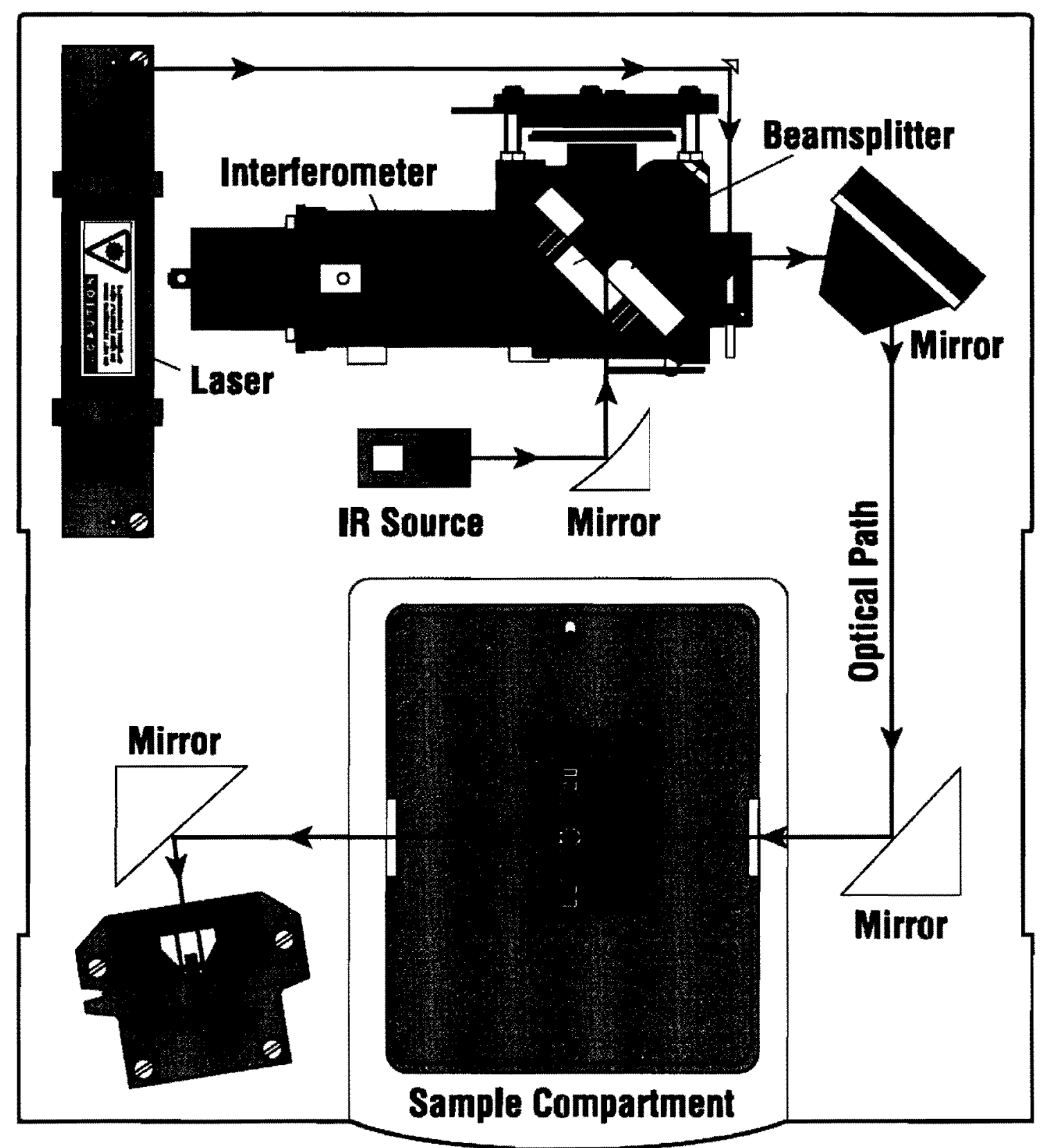




\subsection{Literature background: in situ ATR-IR and dissolution}

In the pharmaceutical industry there are two main oral dosage forms: tablets and capsules.

These are immersed in an aqueous solution, and the concentration of the active ingredient is monitored as a function of time..$^{25}$ Unfortunately, dissolution testing provides limited information on chemical processes that take place within a dissolution vessel. This is due to the limited capabilities of the techniques that are used during dissolution. For instance, most dissolution analysis is carried out using Ultraviolet (UV)/ Visible (Vis) spectroscopy which only gives concentration as a function of time. HPLC is used to separate multi-component drugs but still utilizes UV/Vis detectors as the backbone. One of the challenges the industry is faced with is to increase an understanding of the mechanisms governing dissolution. The current approach relies heavily on a data-driven approach. The health authorities have challenged the pharmaceutical industry to understand dissolution and make the dissolution test more biologically relevant. ${ }^{26,27}$ To have a better understanding of dissolution, dissolution chemists need to explore other instruments and experiments that could give insight into what is happening inside of the vessel. Our research focuses on using ATR mid-infrared spectroscopy as an in situ technique to monitor and study the dissolution of pharmaceutical tablets. Moreover, this research will give more insight into the science behind dissolution. Specifically, this research will attempt to not only monitor dissolution profiles using FTIR but

\footnotetext{
${ }^{25}$ a) Dressman, J. J.; Kramer, J. K., Eds. Pharmaceutical Dissolution Testing; Taylor and Francis: Florida, 2005; b) Hanson, R; Gray, V. Handbook of Dissolution Testing, 3rd ed., Dissolution Technologies: Delaware, 2004.

${ }^{26}$ Tong, C.; D'Souza, S.S.; Parker, J.E.; Mirza, T. Commentary on AAPS workshop - Dissolution testing for the twenty-first century: Linking critical quality attributes and critical process parameters to clinically relevant dissolution. Pharmaceutical Research 2007, 24, 1603-1607.

${ }^{27}$ Tong, C.; Lozano, R.; Mao, Y., Mirza; T., Ldbenberg, R.; Nickerson, B.; Gray, V.; Wang, Q. The value of in Vitro dissolution in drug development: $A$ position paper from the AAPS in Vitro release and dissolution focus group. Pharmaceutical Technology 2009, 33, 52-64.
} 
also try to explain certain reactions (i.e. transformation of a drug from one form to anotherhydrolysis) that are otherwise missed with UV/Vis spectroscopy. Literature research on this topic shows that attempts have been made to study dissolution using near-infrared spectroscopy and infrared imaging.

Attempts have been made to improve the characterization of the controlled release formulations by FT-IR imaging in ATR-IR mode. ${ }^{28}$ Van der Weerd and colleagues described the design and implementation of a new cell, which allows the study of drug release from tablets by macro-FT-IR ATR imaging with a diamond ATR accessory. The tablet formulation can be compacted directly on the ATR crystal. The authors explain that various components in the tablet can be determined FT-IR imaging. The described cell was applied to study a model tablet consisting of hydroxypropyl methylcellulose (HPMC) and caffeine. The study by Van der Weerd and co-workers demonstrate that the amount of drug rapidly decreases due to diffusion. Direct observation of this well know phenomenon is unattainable by dissolution techniques, but FT-IR imaging in ATR mode provides a means to achieve this.

Moreover, Van der Weerd and Kazarian explored FT-IR imaging as a useful application for studying the distribution of different components in the tablet, e.g., drug, polymer and water as a function of time. It was shown that the release profile obtained by FT-IR imaging is comparable to that obtained by the flow-through dissolution test with UV spectroscopic

\footnotetext{
${ }^{28}$ a)Van der Weerd, J.; Kazarian, S. G. Combined approach of FTIR imaging and conventional dissolution tests applied to drug release. Journal Controlled Release 2004, 98, 295-305. b) Van der Weerd, J.; Chan, K. L. A.; Kazarian, S.G. An innovative design of compaction cell for in situ FT-IR imaging of tablet dissolution. Vibrational Spectroscopy 2004, 35(1-2), 9-13.
} 
detection. In addition, FT-IR imaging was also used to investigate the release of a poorly soluble drug from pharmaceutical tablets. ${ }^{29}$

Blanco $^{30}$ and co-workers investigated the use of near-infrared as a tool to study the dissolution profiles in pharmaceutical tablets. Their determination was based on the application of Partial Least-Squares 2 (PLS2) multivariate calibration models to NIR spectra for individual tablets. Marcelo and his colleagues documented that the proposed NIR method provides accurate predictions of dissolution profiles. In fact, the coefficient of correlation between the dissolution profiles obtained with it and a reference method exceeded $0.99(\mathrm{r})$ in all cases, and calibration and prediction errors fell within acceptable ranges $(6-7 \%)$. They conclude that the method allows the entire dissolution profile of intact pharmaceutical tablets to be determined in a simple, clean, expeditious manner without the need to use any reagents or solvents, or the production of any waste.

In addition, Chan ${ }^{31}$ and co-workers investigated the use of FT-IR imaging as a tool to study formulations of ibuprofen in poly(ethylene glycol) (PEG) enabled characterization of the distribution of both polymer and drug in the tablet. The authors attempted to study the mechanism of dissolution and drug release for two tablet preparation methods: mechanical mixing and melt extrusion. The mechanisms of dissolution were compared using this spectroscopic imaging approach and found it to be very informative.

\footnotetext{
${ }^{29}$ a)Van der Weerd, J.; Kazarian S. G. Release of poorly soluble drugs from HPMC tablets studied by FTIR imaging and flow-through dissolution tests. Journal of Pharmaceutical Science 2005, 94, 2096-2109. b) Van der Weerd, J.; Kazarian, S.G. Validation of macroscopic attenuated total reflection-Fourier transform infrared imaging to study dissolution of swelling pharmaceutical tablets. Applied Spectroscopy 2004, 58(12), $1413-1419$.

${ }^{30}$ Blanco, M.; Alcala, M.; Gonzalez, J. M.; Torras, E. Determination of dissolution profiles in intact pharmaceutical tablets by NIR spectroscopy. The Journal of Process Analytical Technology 2006, 3(5), 25-29. ${ }^{31}$ Chan, K. L. A.; Kazarian, S.G. Dissolution of solid dispersions of ibuprofen studied by Fourier Transform infrared imaging. Polymeric Drug Delivery 2006, 203-214.
} 


\section{Dissolution of salicylic acid, acetylsalicylic acid and acetaminophen using In Situ ATR-IR spectroscopy}

This chapter focuses on describing the application of in situ attenuated total reflectanceinfrared spectroscopy (ATR-IR) as a technique for measuring and studying dissolution of pharmaceutical formulations products. The majority of the methods for monitoring dissolutions utilize UV-Vis spectroscopy. For example, aliquots are manually withdrawn and analyzed by UV-Vis spectroscopy or HPLC with UV-Vis detection. However, the sampling process is disruptive to the dissolution profile since removal of aliquots from the vessel disturbs the solution. In addition, there are instruments that allow real-time analysis using in situ UV-Vis probes. For example, fiber optic dissolution testing is used in the industry to monitor pharmaceutical drug product release. Fiber optic dissolution is also used for formulation development. ${ }^{32}$ Formulators are using in situ UV/Vis systems to profile and develop drugs faster. Instead of relying on conventional techniques where dissolutions are conducted manually and analyzed offline, the advantage of the fiber optic system is that it allows for real-time data analysis.

There is interest in the development of new methods that do not require manual sampling. Also, for multi-component formulations it is important to be able to observe the dissolution profile of each active pharmaceutical ingredient (API). Thus, there is also interest in the development of new spectroscopic methods that enable observation of multiple components. The use ATR-IR for analyzing aqueous samples is limited by the relatively high concentration

\footnotetext{
${ }^{32}$ Mirza, T.; Liu, Q.; Vivilecchia, R.; Joshi, Y. Comprehensive validation scheme for in situ fiber optics dissolution method for pharmaceutical drug product testing. Journal Pharmaceutical Science 2009, 98, 10861094.
} 
of analyte required for detection. ${ }^{33}$ Hence, this research investigated the use of ATR-IR as a technique for monitoring and understanding dissolution. Limit of detection (LOD) and limit of quantitation (LOQ) will play important roles in determining the sensitivity of the system. The LOD and LOQ values will be discussed in detail in chapter four of this thesis. Refer to equation 11 and 12 for the limit of detection and limit of quantitation calculations:

(Equation 11)

$$
L O D=3 x\left(\frac{S_{x y}}{b}\right)
$$

(Equation 12)

$$
L O Q=10 x\left(\frac{S_{x y}}{b}\right)
$$

ATR spectroscopy is a sampling technique that is based on molecular vibration and the curvature of light beams when passing through different mediums. An ATR spectrum is generated by transmitting radiation, which can be IR (from $0.1 \times 10^{-5} \mathrm{~cm}$ to $7.5 \times 10^{-5} \mathrm{~cm}$ ), VIS (from $7.0 \times 10^{-5}$ to $4.0 \times 10^{-5} \mathrm{~cm}$ ), or UV (from $4.0 \times 10^{-5} \mathrm{~cm}$ to $2.2 \times 10^{-5} \mathrm{~cm}$ ), through an optical crystal in contact with a sample and then determining what portion of the incident radiation is attenuated by the sample at a particular wavelength.

With ATR sampling the IR beam is directed into a crystal of relatively higher refractive index. The IR beam reflects from the internal surface of the crystal and creates an evanescent wave, which projects orthogonally into the sample in intimate contact with the ATR crystal. Some of the energy of the evanescent wave is absorbed by the sample and the reflected

${ }^{33}$ Regan, F.; Meaney, M.; Vos, J. G.; Maccraith, B. D.; Walsh, J. E. Determination of pesticides in water using [36] 
radiation (some now absorbed by the sample) is returned to the detector. The refractive indices of the crystal and sample are important considerations in the ATR sampling technique. Refer to equation 13:

\section{(Equation 13)}

$$
\theta_{c}=\sin ^{-1}\left(\frac{n_{2}}{n_{1}}\right)
$$

where $\mathrm{n} 2$ is the refractive index of the sample, $\mathrm{nl}$ is the refractive index of the crystal and $\theta \mathrm{c}$ is the critical angle. ATR spectrometry is used extensively in clinical assays, medical diagnostics, and laboratory testing. ${ }^{34}$ Since the depth of penetration for the evanescent wave in ATR spectrometry is shallow, there is a low incidence of Fresnel Reflection. Thus, reliable spectral analysis of murky, semisolid, turbid, and optically dense solutions is possible with ATR spectroscopy. Moreover, the ATR crystal is a relatively chemically resistant $\mathrm{Zn}-\mathrm{Se}$ crystal that can be coated with an additional chemically resistant material which enables IR spectroscopy to be performed in aqueous solution.

Therefore, in situ ATR-IR spectroscopy has the unique potential to simultaneously address problems associated with manual sampling and multi-component analysis discussed above. This is a potentially useful method for dissolution testing. Modern infrared instruments can be equipped with fiber optic probes containing ATR crystals that are chemically robust, provide excellent sensitivity and, with respect to in situ UV-Vis, are not affected by turbidity. In addition, since IR is very sensitive to specific functional groups, it has greater versatility in 
differentiating components in a multi-component mixture than UV-Vis spectroscopy. ${ }^{35}$ In this chapter, the successful development of dissolution tests using in situ ATR-IR spectroscopy to analyze single and multi-component mixtures is described.

Since dissolution testing has evolved into a highly regulated activity in the pharmaceutical industry, health agencies have placed a greater emphasis on regulating dissolution methods. The dissolution test is the only one that can give an in vitro snapshot of how the drug product may behave in vivo. Because of this function, the number of dissolution methods in the United States Pharmacopeia (USP) has grown substantially. Furthermore, the U.S. Food and Drug Administration (FDA) has placed greater importance on the dissolution test. The FDA official website is full of material related to dissolution testing from guidance documents to warning letters. ${ }^{36}$ Thus, the potential impact of a new analytical technique that permits in situ analysis of multiple active ingredients is large.

\subsection{Experimental section}

\subsubsection{Chemical and materials}

Acetaminophen reference material (batch no. 104K0154) was purchased from Sigma-Aldrich (St. Louis, MO, USA). Salicylic acid reference material (batch no. $04708 \mathrm{HE}$ ) was purchased from Sigma-Aldrich (St. Louis, MO, USA). Acetaminophen tablets (Tylenolß batch no. SLA175) were purchased from a local pharmacy. Excedrinß Caplets (Back and Body Brand, Lot \#10067371: Acetaminophen $250 \mathrm{mg}$, Aspirin $250 \mathrm{mg}$ ) were purchased from a local pharmacy. Salicylic acid calibrator tablets (USP batch no. Q0D200) were purchased from the

\footnotetext{
${ }^{35}$ Skoog, D.; Holler J.; Crouch S. Principles of Instrumental Analysis, 6th ed.; Thomson Books, 2007; pp. 455480 .

${ }^{36}$ United States Food and Drug Administration Home Page. http://www.fda.gov/ (accessed May 1, 2011)
} 
USP. Methanol, acetone and acetonitrile (HPLC grades) were purchased from PharmacoAaper. Sodium hydroxide (batch no. 064214BH), used to prepare the $\mathrm{pH}$ buffered solutions, was purchased from Sigma-Aldrich (St. Louis, MO, USA). These analytes were selected because of their immediate release properties and high solubilities in phosphate buffers. Potassium phosphate monobasic (batch no. 103K0060), used to prepare the $\mathrm{pH}$ buffered solutions, was purchased from Sigma-Aldrich. Glacial acetic acid was purchased from Sigma Aldrich (St. Louis, MO, USA). All solutions were prepared using water treated by a Milli-Q Millipore purification system. All purified water aliquots have resistivity of not less than 18 MOhm-cm ${ }^{-1}$.

\subsubsection{Instrumentation}

Samples were tested using Mettler Toledo's iC10 FT-IR system using a fiber optic probe equipped with a $1 \mathrm{~mm}$ diamond coated ATR probe. The IR system was operated by Mettler Toledo's iC IR version 3.0 or 4.0 software. $\mathrm{pH}$ determinations were carried out using a $\mathrm{pH}$ meter from VWR (model no. Symphony SB70P). HPLC analysis was carried out using two systems: Hewlett Packard 1050 (operated by ChemStation) and Waters 2695 (operated by Empower). All UV measurements were carried out using a Hewlett Packard UV instrument (model no. 8452A diode array). The UV instrument was operated using HP's Softer-Olis Spectralworks. All manual dissolutions were tested using a Vankel Dissolution Bath (model no. 700). All analytical weight measurements were carried out using Mettler Toledo's DeltaRange and AG204 DeltaRange top loading balance. 


\subsubsection{Buffered solutions}

The $\mathrm{pH} 5.8(200 \mathrm{mM})$ and $7.4(50 \mathrm{mM})$ phosphate buffered solutions were prepared with monobasic potassium phosphate in accordance with the USP. As described in the USP, the detailed procedure for preparing these buffers is shown below. ${ }^{37,38}$

\section{Phosphate Buffered Solution:}

The volumes shown in the table are for $200 \mathrm{~mL}$ of buffer solution. Note: Where water is specified for solution or dilution of test substances in $\mathrm{pH}$ determinations, use carbon dioxidefree water.

- Sodium Hydroxide Solution (0.2 M) - Dissolve $4.0 \mathrm{~g}$ of sodium hydroxide in water to make $100 \mathrm{~mL}$.

- Monobasic Potassium Phosphate Solution (0.2 M) - Dissolve $27.22 \mathrm{~g}$ of monobasic potassium phosphate $\left(\mathrm{KH}_{2} \mathrm{PO}_{4}\right)$ in water, and dilute with water to $1000 \mathrm{~mL}$.

Table 2-1

\section{Phosphate buffer preparations as per United States Pharmacopeia (USP)}

\begin{tabular}{|c|c|c|c|c|c|c|c|c|c|c|c|c|}
\hline \multicolumn{13}{|c|}{ Composition of Standard Buffer Solutions } \\
\hline \multicolumn{13}{|c|}{ Phosphate Euffer } \\
\hline $\begin{array}{l}\text { Place } 50 \mathrm{~m} \text {. of } \\
\text { sclution, then ad }\end{array}$ & & & & & & & & & & & & \\
\hline $\mathrm{pH}$ & 5.8 & 6.0 & 6.2 & 6.4 & 6.6 & 68 & 70 & 72 & 7.4 & 76 & 7.8 & 80 \\
\hline $0,2 \mathrm{M} \mathrm{NaOH}_{\mathrm{mL}} \mathrm{mL}$ & 36 & 5.6 & 81 & 116 & 164 & 22.4 & 29.1 & 34.7 & 39.1 & 42.4 & 445 & 461 \\
\hline
\end{tabular}

\subsubsection{Dissolution experiments}

Single component analysis: $300 \mathrm{mg}$ salicylic acid tablets were tested in $\mathrm{pH} 7.4$ phosphate buffered solutions. Dissolution was conducted using a vessel volume of $500 \mathrm{~mL}$ and at ambient temperature. All dissolutions were conducted using USP Apparatus II (paddles) with an agitation speed of $100 \mathrm{rpm}$. The dissolution run time was seven hours and thirty minutes.

\footnotetext{
${ }^{37}$ US Pharmacopoeia \& National Formulary- USP Monograph: Salicylic Acid Tablets, USP [Online], http:/www.usp.org/ (accessed May 1, 2011).
} 
Multi-tablet analysis: $300 \mathrm{mg}$ salicylic acid and $500 \mathrm{mg}$ acetaminophen tablets were tested in $\mathrm{pH} 5.8$ phosphate buffered solutions. Dissolution was conducted using a vessel volume of 500 $\mathrm{mL}$ and at ambient temperature. All dissolutions were conducted using USP Apparatus II (paddles) with an agitation speed of $50 \mathrm{rpm}$ (a slower rpm was chosen to enable a slower rate of dissolution to help distinguish the components). The dissolution run time was six hours.

Multi-component analysis: Three Excedrin $\mathbb{B}$ tablets, each composed of $250 \mathrm{mg}$ aspirin (acetylsalicylic acid) and $250 \mathrm{mg}$ acetaminophen were tested in $\mathrm{pH} 7.4$ phosphate buffered solutions. Dissolution was conducted using a vessel volume of $500 \mathrm{~mL}$ and at ambient temperature. All dissolutions were conducted using USP Apparatus II (paddles) with an agitation speed of $100 \mathrm{rpm}$. The dissolution run time was one hour and thirty minutes.

\subsubsection{HPLC analysis}

The single-component analysis experiment was performed using the following HPLC parameters: HP $1050 \mathrm{HPLC}$, Waters Symmetry $300 \mathrm{C} 185 \mu \mathrm{m}$ column $(4.6 \times 50 \mathrm{~mm})$, mobile phase was $60 / 40 / 1$ (water/ methanol/glacial acetic acid), flow rate was $2.0 \mathrm{~mL} / \mathrm{min}$, isocratic mode, injection volume was $5 \mu \mathrm{L}$ and absorbance was set at $296 \mathrm{~nm}$.

The multi-tablet analysis experiment was analyzed using the following HPLC parameters: Waters 2695, Waters Symmetry Shied RP C18 $3 \mu \mathrm{m}$ column $(3.5 \times 50 \mathrm{~mm})$, mobile phase was 70/30/1 (water/ acetonitrile/trifluoroacetic acid (TFA)), flow rate was $1.5 \mathrm{~mL} / \mathrm{min}$, isocratic mode, injection volume was $5 \mu \mathrm{L}$ and absorbance was set at $296 \mathrm{~nm}$.

\footnotetext{
${ }^{38}$ US Pharmacopoeia \& National Formulary- USP Monograph: Acetaminophen Tablets, USP [Online], http://www.usp.org/ (accessed May 1, 2011).
} 
The multi-component analysis experiment was performed using the following HPLC parameters: HP 1050 HPLC, Phenomenex Intersil ODS C18 $5 \mu \mathrm{m}$ column $(4.6 \times 150 \mathrm{~mm})$, mobile phase was 60/40/1 (methanol/ water/ trifluoroacetic acid (TFA)), flow rate was 1.0 $\mathrm{mL} / \mathrm{min}$, isocratic mode, injection volume was $25 \mu \mathrm{L}$ and absorbance was set at $280 \mathrm{~nm}$.

\subsubsection{ATR-IR analysis}

The ReactIR ${ }^{\mathrm{TM}}$ iC10 FTIR instrument is composed of a Mercury-Cadmium-Telluride ("MCT") detector (liquid nitrogen cooled) and the FiberConduit ${ }^{\mathrm{TM}}$. When sample measurements must be made at high speed or when IR throughput is low, the highly sensitive mercury cadmium telluride (MCT) detector provides the ability to scan faster than a DLaTGS detector while maintaining a constant IR response. The FiberConduit ${ }^{\mathrm{TM}}$ is comprised of flexible IR transparent silver chloride/silver bromide optical fibers. The fiber optic probe interface (AgX $9.5 \mathrm{~mm} \times 1.5 \mathrm{~m}$ Fiber (Silver Halide)) contains a diamond tip-DiComp ATR crystal. The resolution was set to 4 wavenumbers. The optical range used by the system is: $1900 \mathrm{~cm}^{-1}$ to $650 \mathrm{~cm}^{-1}$. The gain adjustment was set to normal (1x) and the apodization method was set to Happ-Genzel. The system uses compressed air (house air, filtered and dehumified) to purge the optics.

For the single component testing, data treatment was carried out using the following methodology: The data was first subjected to baseline correction. An absorption band at 1388 $\mathrm{cm}^{-1}$ was selected for salicylic acid. The height was calculated using a baseline band correction set at $1370 \mathrm{~cm}^{-1}$. The ATR-IR system was configured to collect spectra every five minutes. 
For multi-tablet testing, the data was first subjected to baseline correction. An absorption band at $1388 \mathrm{~cm}^{-1}$ and baseline band at $1370 \mathrm{~cm}^{-1}$ were selected to calculate the peak height for salicylic acid. An absorption band at $1246 \mathrm{~cm}^{-1}$ and a baseline band of $1276 \mathrm{~cm}^{-1}$ were selected to calculate the peak height for acetaminophen. The ATR-IR system was configured to collect spectra every five minutes.

For multi-component testing, the data was first subjected to baseline correction. An absorption band at $1388 \mathrm{~cm}^{-1}$ and a two-point baseline set at $1370 \mathrm{~cm}^{-1}$ and $1414 \mathrm{~cm}^{-1}$ were selected to calculate the peak area for aspirin (acetylsalicylic acid). An absorption band at $1246 \mathrm{~cm}^{-1}$ and a two-point baseline set at $1217 \mathrm{~cm}^{-1}$ and $1265 \mathrm{~cm}^{-1}$ were selected to calculate the peak area for acetaminophen. The ATR-IR system was configured to collect spectra every three minutes.

For all ATR-IR experiments, 256 scans were collected and co-added for each spectral point. On average, every spectral point, took about two-minutes to complete. For all testing, the calculated peak response was subjected to mathematical smoothing using the $\mathrm{iC}_{10}$ software. The data was compared to reference standards measurements collected prior to start of the linearity and dissolution experiments. Dissolution data was plotted vs. time and all dissolution experiments were allowed to equilibrate in the buffer for at least half an hour prior to the start of a dissolution experiment. 


\subsection{Results and discussion}

\subsubsection{Single-component analysis}

\subsubsection{Linearity results for salicylic acid using $\mathrm{pH} 7.4$ phosphate buffer}

Salicylic acid was thoroughly studied in $\mathrm{pH} 7.4$ phosphate buffered solution as recommended by the USP. Since salicylic acid has a pKa of 2.97 it is almost completely ionized at physiologic $\mathrm{pH}$ 7.4. Thus, the ratio of salicylic acid: salicylate is approximately 1:25,000. During the method development phase, linear dilutions of salicylic acid reference standards were prepared and analyzed using ATR-IR spectroscopy. The linearity experiments served two purposes; 1) they determined whether IR spectroscopy provided a linear response to the different concentrations of salicylic acid, and, 2) whether IR spectroscopy can be used for very low concentration levels of salicylic acid. Initially, eight levels of salicylic acid standards were prepared. Based on the IR spectra obtained, salicylic acid has an IR frequency of interest at $1388 \mathrm{~cm}^{-1}$ (Figure 2-1). The linearity experiments were further analyzed using linear regression. Based on the analysis, it was determined that salicylic acid had excellent linear

correlation using IR spectroscopy with a 0.997 correlation coefficient $\left(\mathrm{r}^{2}\right)$. Linear regression for salicylic acid was calculated using the IR absorption band at $1388 \mathrm{~cm}^{-1}$ and subtracting the baseline absorption band at $1370 \mathrm{~cm}^{-1}$. A chart of the digitized data is attached in Appendix [A]. Since the IR system resolution was set to four, large data sets were obtained during the analysis. An example of the digitized data can be found in the appendix. 
Seton Hall University

Figure 2-1 Salicylic acid spectra at different concentrations in $\mathrm{pH} 7.4$ phosphate buffered solution.

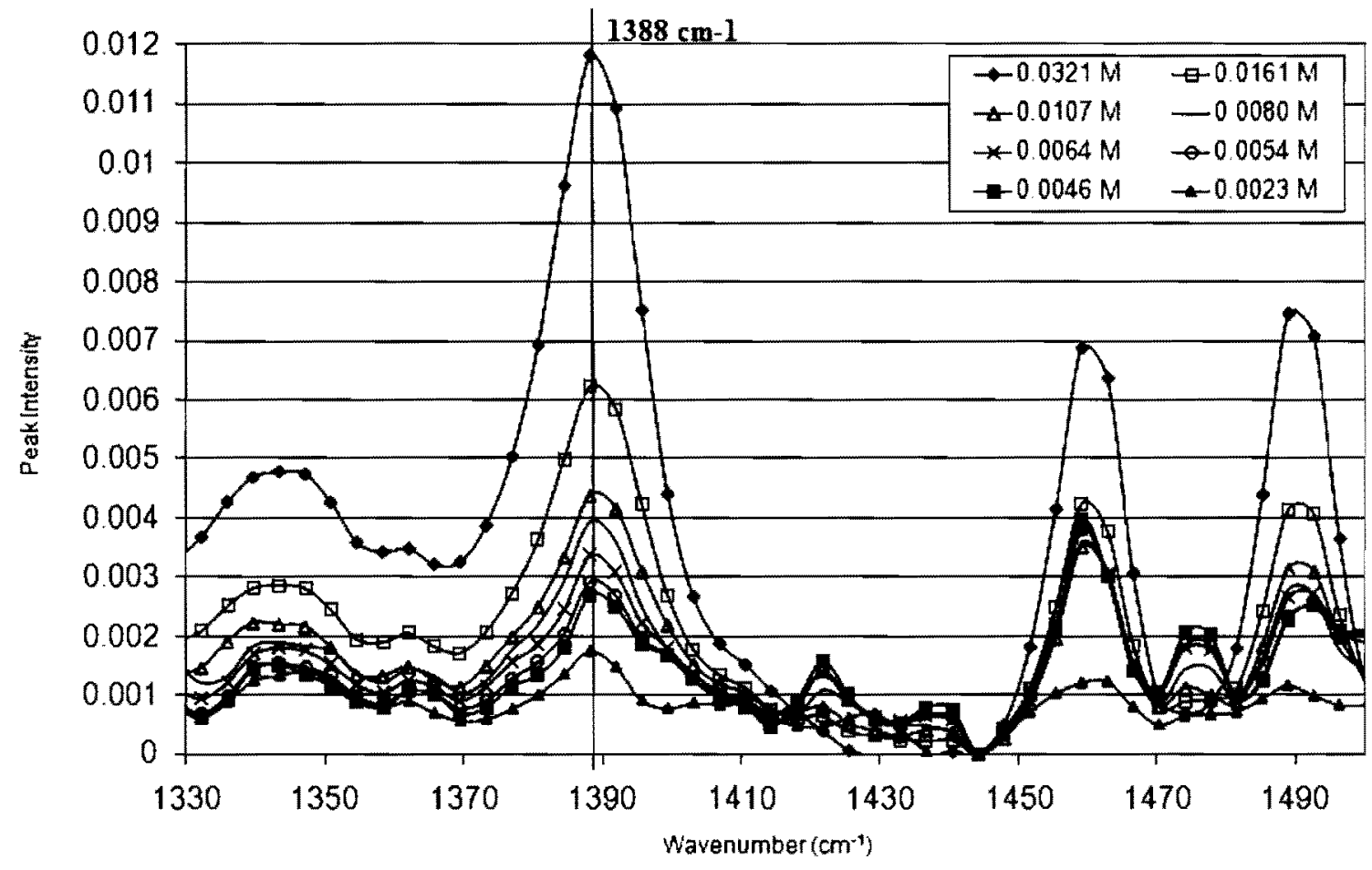


Seton Hall University

Table 2-2 Method development summary for Salicylic Acid (Linearity and Dissolution results)

\begin{tabular}{|c|c|c|}
\hline Method development parameter & $\begin{array}{l}\text { Typical Pharmaceutical } \\
\text { Acceptance criteria }\end{array}$ & Results \\
\hline $\begin{array}{l}\text { Accuracy (mean) } \\
\text { - Difference between IR and HPLC }\end{array}$ & Report value & $2.1 \%$ \\
\hline $\begin{array}{l}\text { Linearity }(n \geq 6) \\
\text { - Correlation coefficient } \\
\text { - Range(Linearity experiment) }\end{array}$ & $\begin{array}{l}r \geq 0.990 \\
\text { Specify range }\end{array}$ & $\begin{array}{l}0.997 \\
0.016-0.1130 \mathrm{mg} / \mathrm{m}\end{array}$ \\
\hline Limit of detection (LOD) & Specify LOD & $0.02 \mathrm{mg} / \mathrm{ml}$ \\
\hline Limit of quantitation (LOQ) & Specify $L O Q$ & $0.08 \mathrm{mg} / \mathrm{ml}$ \\
\hline Precision (\%RSD) & $\begin{array}{l}\text { Relative standard deviation } \\
(n \geq 6), \leq 2.0 \%\end{array}$ & $0.2 \%$ \\
\hline
\end{tabular}




\subsubsection{Dissolution results for salicylic acid using $\mathrm{pH}$ 7.4 phosphate buffer}

The next part of method development phase was to determine the accuracy of ATR-IR spectroscopy as a technique for measuring dissolution rates of a drug. This was determined by comparing the IR spectroscopy results with HPLC results. Although the USP methods typically recommend vessel volumes of $900 \mathrm{~mL}$, the dissolution vessels were filled with 500 $\mathrm{mL}$ of $\mathrm{pH} 7.4$ phosphate buffer in order to increase the signal-to-noise ratio. The purpose of this change was to detect very low concentrations (below $10 \%$ ) of the active drug. Practically the laboratory is not interested in seeing $10 \%$ levels, but rather higher concentrations. Therefore, in order to test the limits of the system, the lower dissolution vessel volumes were used. Next, the paddle was lowered to the USP recommended position $(25 \pm 2 \mathrm{~mm}$ from the bottom of the vessel) and rotated at $100 \mathrm{rpm}$ at ambient temperature. To minimize hydrodynamic effects, the ATR probe was inserted approximately $5 \mathrm{~mm}$ below the surface of the medium. ${ }^{39}$ The first thirty-minutes were used to establish the spectral baseline, the shaft was momentarily stopped and one salicylic acid $(300 \mathrm{mg})$ tablet was dropped into the vessel. Rotation was resumed and IR data were acquired every five minutes for a period of seven hours. During this time, aliquot samples were removed and stored in HPLC vials for subsequent analysis. As shown in Figure 2-2, the results from the IR and HPLC systems match extremely well and the relative accuracy is within $\pm 2 \%$.

\footnotetext{
${ }^{39}$ Mauger J. W. Physicochemical and fluid mechanical principles applied to dissolution testing. Dissolution Technologies, 1996, 3, 7-11.
} 
Figure 2-2 Comparison of in situ ATR-IR and HPLC methods for dissolution of a salicylic acid tablet

The dissolution plot below represents \% dissolved of salicylic acid vs. time. This particular experiment was carried out over seven hours. The light blue diamond data points represent the ATR-IR results whereas the red squares represent the HPLC results. Moreover, as observed in the figure below, the IR data at approximately seven hours appears to be noisy. This is probably due to disturbance of the fiber optic probe during the HPLC sample collections at the seven-hour time points.

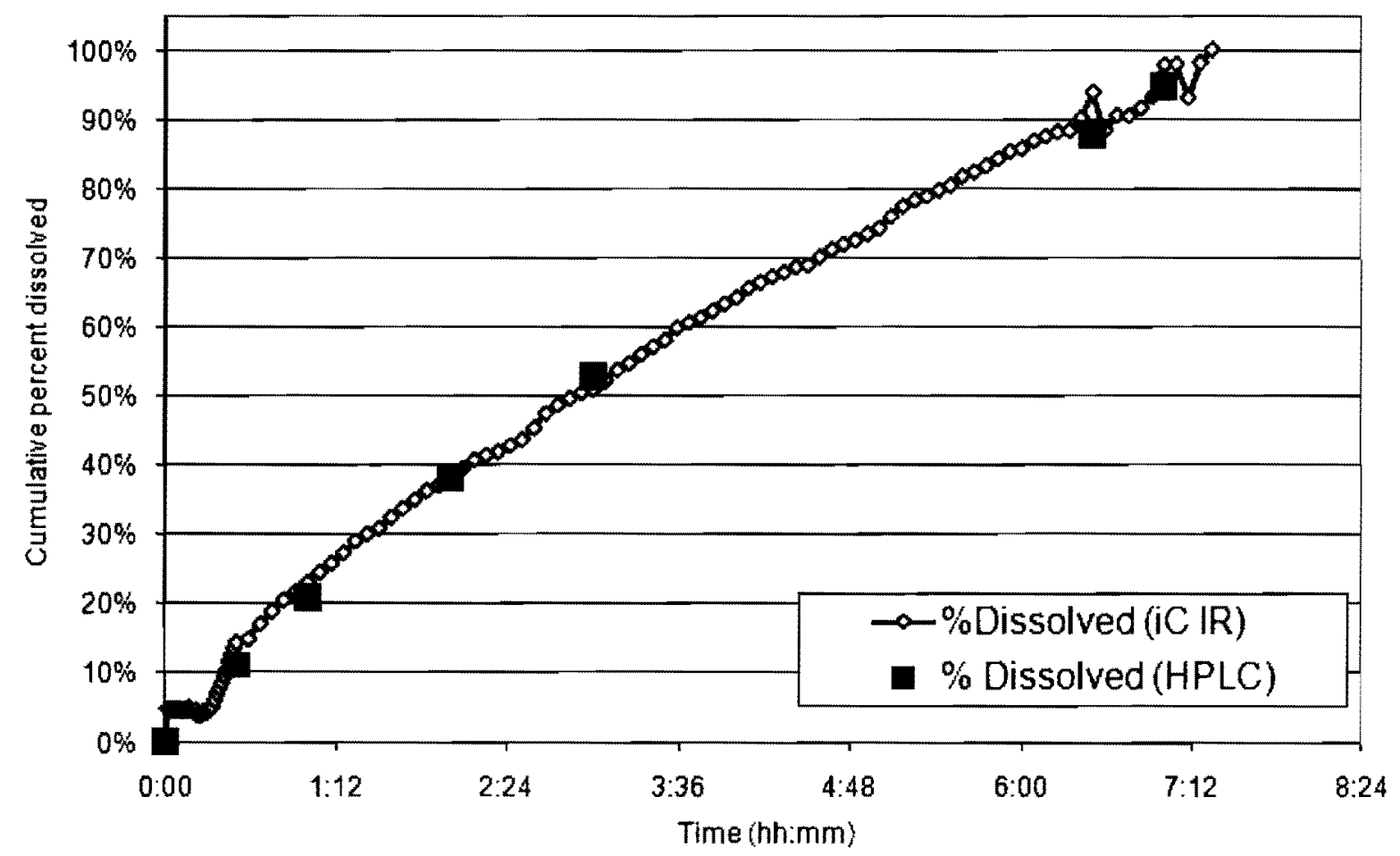


Table 2-3 \% Dissolved of salicylic acid: Absolute \% dissolved difference between ATR-IR and HPLC results

This data represents the dissolution results for salicylic acid from Figure 2-2. The ATR-IR data was compared to the HPLC data and the absolute difference between both methods was calculated. The average difference was also calculated.

\begin{tabular}{|c|c|c|c|}
\hline Time (h:mm) & $\begin{array}{c}\text { Salicylic acid (HPLC) } \\
\text { (\% Dissolved) }\end{array}$ & $\begin{array}{c}\text { Salicylic acid (ATR-IR) } \\
\text { (\% Dissolved) }\end{array}$ & $\begin{array}{c}\text { Absolute Difference } \\
\text { (\%) }\end{array}$ \\
\hline $0: 00$ & $0.0 \%$ & $0.0 \%$ & $0.0 \%$ \\
\hline $0: 30$ & $11.1 \%$ & $14.4 \%$ & $3.3 \%$ \\
\hline $1: 00$ & $20.9 \%$ & $23.1 \%$ & $2.2 \%$ \\
\hline $2: 00$ & $38.2 \%$ & $38.0 \%$ & $-0.1 \%$ \\
\hline $3: 00$ & $52.9 \%$ & $50.9 \%$ & $-2.0 \%$ \\
\hline $6: 00$ & $87.8 \%$ & $93.8 \%$ & $6.0 \%$ \\
\hline $7: 00$ & $94.8 \%$ & $97.8 \%$ & $3.0 \%$ \\
\hline
\end{tabular}




\subsubsection{Multi-tablet analysis}

\subsubsection{Linearity results for salicylic acid and acetaminophen in pH 5.8 phosphate buffer}

The USP monograph specifies $\mathrm{pH} 5.8$ phosphate buffer as the medium of choice for acetaminophen. Thus, acetaminophen and salicylic acid were thoroughly studied in $\mathrm{pH} 5.8$ phosphate buffer. As expected, regression analysis for salicylic acid indicated an excellent linear correlation $\left(r^{2}=0.994\right)$ of IR peak intensity vs. concentration. For acetaminophen, at low concentrations $(\leq 0.11 \mathrm{mg} / \mathrm{ml}, 0.7 \mathrm{mM})$, the relationship between absorbance and concentration is effectively linear $\left(r^{2}=0.998\right)$. However, to our surprise, non-linear behavior for both IR and UV-Vis methods was observed at higher concentrations. As shown in Figure 2-3, both methods can be modeled using second-order polynomial equations. ${ }^{40}$

\footnotetext{
${ }^{40}$ Dejaegher, B.; Bloomfield, M.S.; Smeyers-Verbeke, J.; Heyden, Y. Validation of a Fluorimetric Assay for 4 Aminophenol in Paracetamol Formulations. Talanta 2008, 75, 258-265.
} 
Figure 2-3 Calibration curves of acetaminophen standards at pH 5.8: UV/Vis absorbance at $296 \mathrm{~nm}$ and IR peak intensity at $1246 \mathrm{~cm}^{-1}$

Acetaminophen in $\mathrm{pH} 5.8$ buffer is linear at the lower concentrations. During the early stage of dissolution, the concentration (C) of the active drug is much lower than the saturation concentration (Cs). However, as time progresses the solution becomes saturated at which point the drug will compete for solution space. Moreover, as more molecules of acetaminophen are in solution, more hydrogen bonding (between carbonyl and hydroxyl groups) will occur between the molecules potentially causing dimerization in solution.

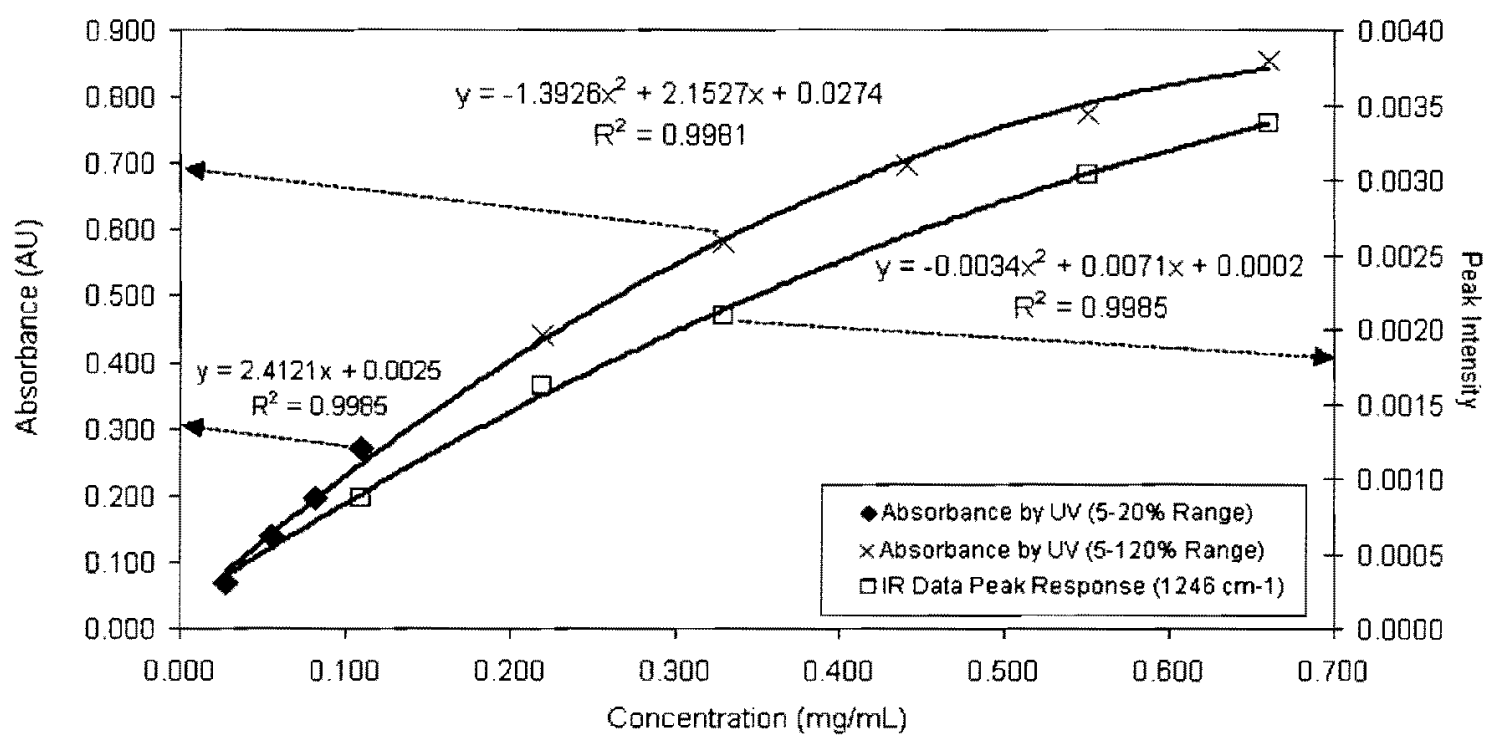




\subsubsection{Dissolution results for salicylic acid and acetaminophen in pH 5.8 phosphate buffer}

To demonstrate the ability of in situ ATR-IR to successfully monitor dissolution of two different tablets, the dissolution profile of salicylic acid and acetaminophen tablets was determined by simultaneously adding one tablet of each and monitoring the dissolution rate of both components over seven hours.

Although salicylic acid is not used clinically, it serves as an USP recommended standard for a slowly eroding tablet and acetaminophen serves as an example of a tablet that disintegrates. As shown in the figure below, the in situ ATR-IR method is clearly able to distinguish the dissolution of each component. For example, the acetaminophen tablet disintegrates and releases acetaminophen more rapidly than the salicylic acid tablet which slowly erodes over time. Thus, in situ ATR-IR method is successful in determining the dissolution profile of a two component system.

To compare the in situ ATR-IR method with HPLC and UV-Vis methods, aliquots were taken throughout the experiment and analyzed. The results from the in situ ATR-IR and HPLC systems are overlaid in Figure 2-4 and an excellent correlation is observed between the two methods. In addition, samples were analyzed using a UV/Vis instrument. Since both salicylic acid and acetaminophen absorb at approximately $296 \mathrm{~nm}(\mathrm{pH} 5.8$ ) it will not be ideal to monitor the dissolution of these two components using UV/Vis alone. Moreover, extensive mathematical equations will be required to solve simultaneous equations in order to differentiate the two active drugs. 
Figure 2-4 Simultaneous dissolution of acetaminophen and salicylic acid tablets: in situ ATR-IR vs. HPLC

The dissolution plot below represents \% dissolved of individual salicylic acid and acetaminophen tablets vs. time. Moreover, two separate tablets were inserted into a single vessel during the analysis. The dissolution was carried out at $\mathrm{pH} 5.8$ phosphate buffer as suggested by the USP. This particular experiment was carried out over six hours. The red squares and yellow diamonds represent the HPLC data. As observed, the ATR-IR and HPLC results are very similar at the specified time-points shown below. Since acetaminophen tablets are designed to disintegrate rapidly, the drug release quickly as observed in the plot. Since salicylic acid is an eroding (slowly releasing) tablet, the drug releases slower as observed in the plot. In addition, the dissolution was carried out using an agitation speed of RPM.

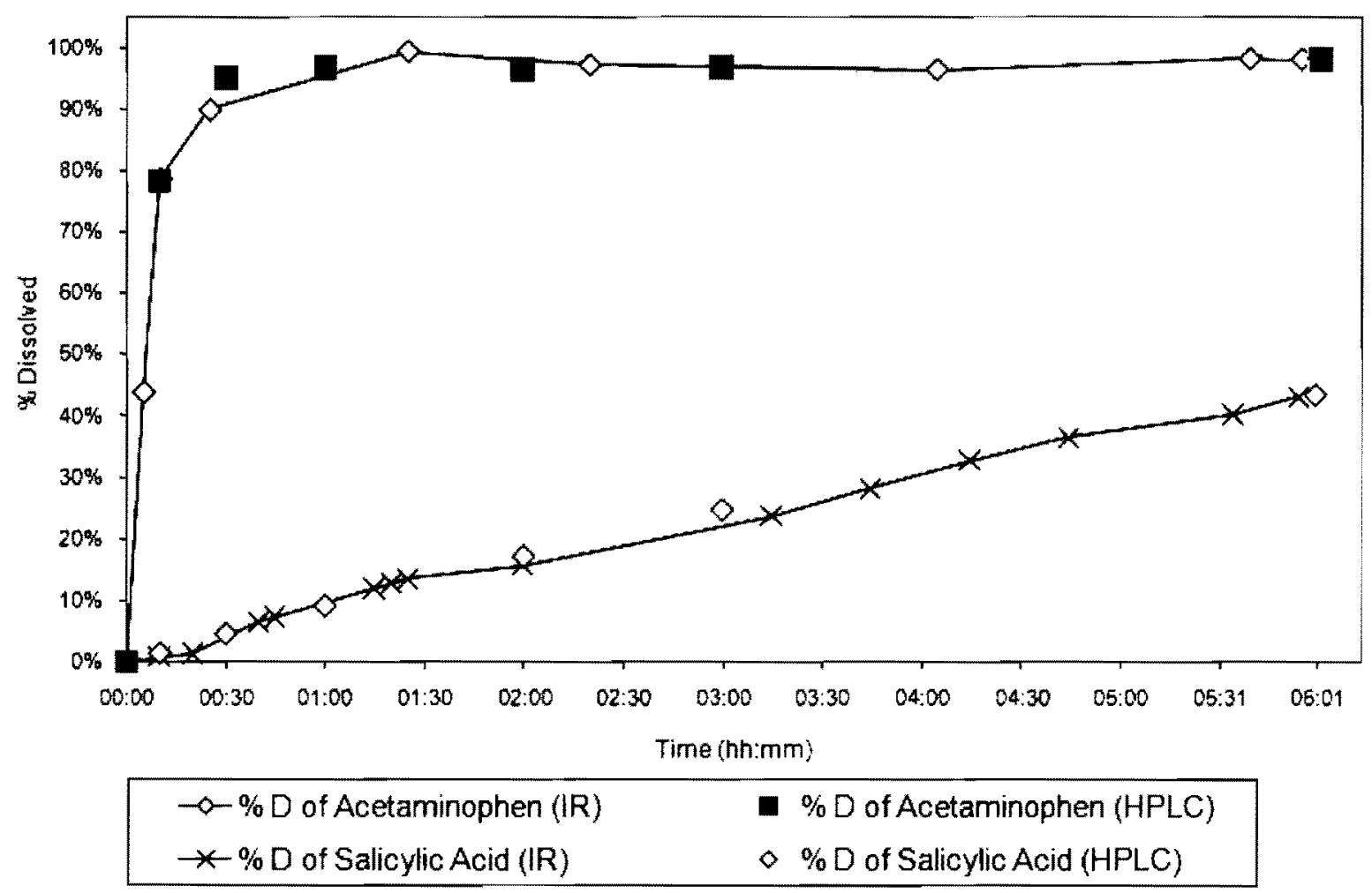




\subsubsection{Multi-component analysis}

\subsubsection{Dissolution results for Excedrin $®$ caplets (aspirin and acetaminophen) in pH 7.4 phosphate buffer}

To demonstrate the ability of in situ ATR-IR to successfully monitor multiple components within the same tablet, dissolution testing was carried out on Excedrin® caplets. The label claims for the Excedrin® caplet are acetylsalicylic acid $(250 \mathrm{mg})$ and acetaminophen $(250$ mg) actives. The release profile was determined by simultaneously adding three Excedrin $(\mathbb{B}$ caplets to a $500 \mathrm{~mL}$ vessel volume containing $\mathrm{pH} 7.4$ phosphate buffer. Three caplets were necessary to ensure satisfactory signal to noise ratio. Refer to Figure 2-5 for ATR-IR spectra for Excedrin ${ }^{\circledR}$ caplets. The dissolution rates of both components were monitored over 1.5 hours. Based on the data, it was determined that acetylsalicylic acid and acetaminophen were fully released within 1.5 hours as shown in Figure 2-6. In situ ATR-IR method is clearly able to distinguish the dissolution the acetylsalicylic acid and acetaminophen components.

To compare the in situ ATR-IR method with HPLC, aliquots were taken throughout the experiment and analyzed. The results from the in situ ATR-IR and HPLC systems are overlaid in Figure 2-6 and an excellent correlation is observed between the two methods. Refer to Table 2-4 to Table 2-7 for statistical values. Overall, this set of experiments indicates the versatility of in situ ATR-IR for dissolution testing as it allows automated observation of a multi-component system without manual sampling and by successfully tracking the dissolution behavior of two of the major components. 
Figure 2-5 ATR-IR spectra of Excedrin $®$ caplets at different time-points during dissolution in $\mathrm{pH} 7.4$ phosphate buffered solution

ATR-IR spectra for Excedrin $\otimes$ caplet (acetaminophen and acetylsalicylic acid) in pH 7.4 phosphate buffer solution. Acetaminophen was calculated using wavenumber $1246 \mathrm{~cm}^{-1}$ and acetylsalicylic acid was calculated using $1388 \mathrm{~cm}^{-1}$.

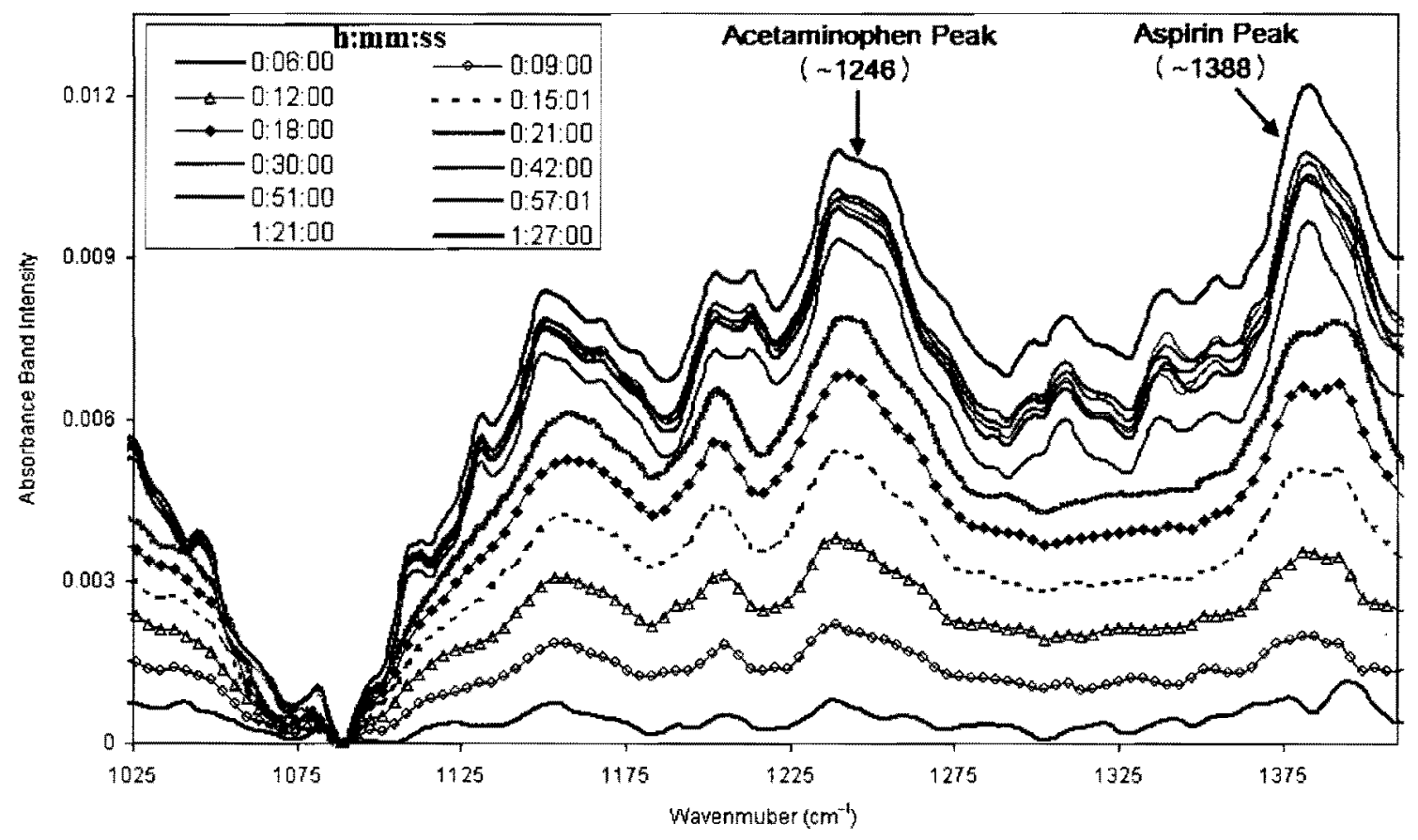


Figure 2-6 Dissolution of acetylsalicylic acid and acetaminophen in Excedrin® caplets: in situ ATR-IR vs. HPLC

The figure below represents a plot of Excedrin®) (acetaminophen and acetylsalicylic acid \% dissolved) vs. time. The blue squares and yellow triangles represent the HPLC data points. As observed, the two techniques (IR and HPLC) correlate very well. Refer to Table 2-4 for more details.

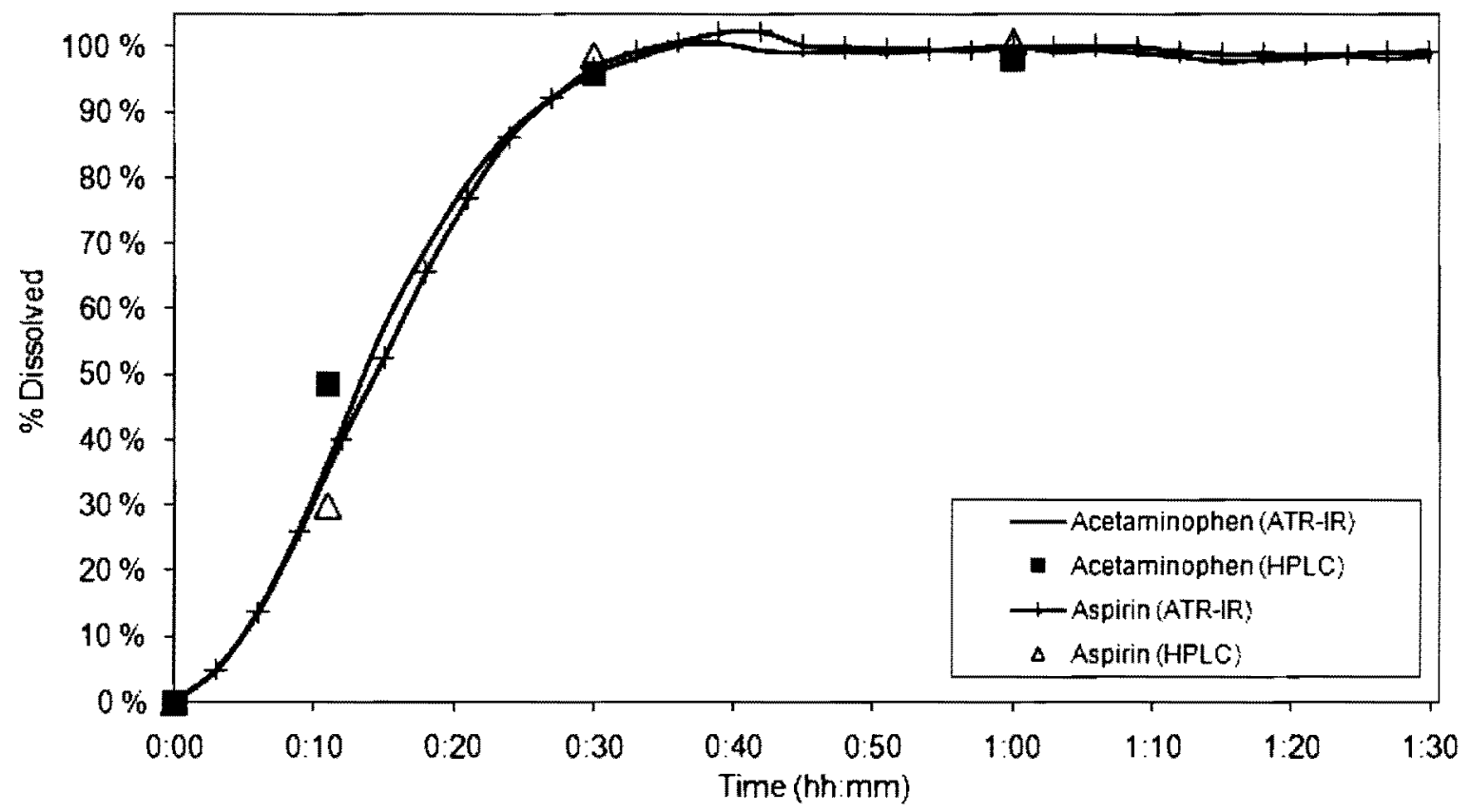


Table 2-4

Dissolution of acetylsalicylic acid (aspirin) and acetaminophen in Excedrin® caplets: ATR-IR raw data

Raw data results for the ATR-IR spectra of Excedrin $\otimes$ caplets.

\begin{tabular}{|c|c|c|}
\hline Time (h:mm:ss) & $\begin{array}{c}\text { Acetaminophen (ATR-IR) } \\
\text { (\% Dissolved) }\end{array}$ & $\begin{array}{c}\text { Aspirin (ATR-IR) } \\
\text { (\% Dissolved) }\end{array}$ \\
\hline $0: 00: 00$ & 0.0 & 0.0 \\
\hline $0: 03: 00$ & 4.8 & 4.9 \\
\hline $0: 05: 59$ & 13.7 & 13.6 \\
\hline $0: 08: 59$ & 26.5 & 25.9 \\
\hline $0: 11: 59$ & 41.6 & 39.8 \\
\hline $0: 15: 00$ & 57.3 & 52.5 \\
\hline$\overline{0}: 17: 59$ & 69.0 & 65.6 \\
\hline $0: 20: 59$ & 79.2 & 76.7 \\
\hline $0: 23: 59$ & 86.8 & 86.1 \\
\hline $0: 27: 00$ & 92.1 & 92.0 \\
\hline $0: 29: 59$ & 95.8 & 96.8 \\
\hline $0: 32: 59$ & 98.2 & 99.2 \\
\hline $0: 35: 59$ & 100.1 & 100.6 \\
\hline $0: 38: 59$ & 100.4 & 102.1 \\
\hline $0: 41: 59$ & 99.3 & 102.2 \\
\hline $0: 44: 59$ & 99.0 & 100.2 \\
\hline $0: 48: 00$ & 99.2 & 99.9 \\
\hline $0: 50: 59$ & 98.9 & 99.7 \\
\hline $0: 53: 59$ & 99.3 & 99.5 \\
\hline $0: 57: 00$ & 99.6 & 99.3 \\
\hline $0: 59: 59$ & 100.0 & 100.0 \\
\hline 1:02:59 & 99.2 & 100.0 \\
\hline $1: 06: 00$ & 99.3 & 100.1 \\
\hline $1: 08: 59$ & 98.9 & 99.9 \\
\hline $1: 12: 00$ & 98.4 & 99.2 \\
\hline$\overline{1}: 14: 59$ & 97.6 & 98.9 \\
\hline $1: 17: 59$ & 97.9 & 98.9 \\
\hline $1: 20: 59$ & 98.1 & 98.8 \\
\hline $1: 24: 00$ & 98.6 & 98.8 \\
\hline $1: 26: 59$ & 98.0 & 99.2 \\
\hline $1: 30: 00$ & 98.6 & 99.2 \\
\hline
\end{tabular}


Table 2-5 Dissolution of acetylsalicylic acid (aspirin) and acetaminophen in Excedrin® caplets: HPLC raw data

Raw HPLC data results for Excedrin $\otimes$ caplets.

\begin{tabular}{|c|c|c|}
\hline Time (h:mm:ss) & $\begin{array}{c}\text { Acetaminophen (HPLC) } \\
\text { (\% Dissolved) }\end{array}$ & $\begin{array}{c}\text { Aspirin (HPLC) } \\
\text { (\% Dissolved) }\end{array}$ \\
\hline $0: 00: 00$ & 0.0 & 0.0 \\
\hline $0: 11: 00$ & 48.4 & 29.9 \\
\hline $0: 30: 00$ & 95.7 & 98.6 \\
\hline $1: 00: 00$ & 98.0 & 100.7 \\
\hline
\end{tabular}

Table 2-6 \% Dissolved of acetaminophen: Difference between ATR-IR and HPLC results

\begin{tabular}{|c|c|c|c|}
\hline $\begin{array}{c}\text { Time } \\
\text { (h:mm:ss) }\end{array}$ & $\begin{array}{c}\text { Acetaminophen (HPLC) } \\
\text { (\% Dissolved) }\end{array}$ & $\begin{array}{c}\text { Acetaminophen (ATR-IR) } \\
\text { (\% Dissolved) }\end{array}$ & Absolute Difference \\
\hline $0: 00: 00$ & 0.0 & 0.0 & 0.0 \\
\hline $0: 11: 00$ & 48.4 & 41.6 & 6.8 \\
\hline $0: 30: 00$ & 95.7 & 95.8 & -0.1 \\
\hline $1: 00: 00$ & 98.0 & 100.0 & -2.0 \\
\hline
\end{tabular}

Table 2-7 \% Dissolved acetylsalicylic acid (aspirin) analysis: Difference between ATR-IR and HPLC results

\begin{tabular}{|c|c|c|c|}
\hline $\begin{array}{c}\text { Time } \\
\text { (h:mm:ss) }\end{array}$ & $\begin{array}{c}\text { Aspirin (HPLC) } \\
\text { (\% Dissolved) }\end{array}$ & $\begin{array}{c}\text { Aspirin (ATR-IR) } \\
\text { (\% Dissolved) }\end{array}$ & Absolute Difference \\
\hline $0: 00: 00$ & 0.0 & 0.0 & 0.0 \\
\hline $0: 11: 00$ & 29.9 & 39.8 & -9.9 \\
\hline $0: 30: 00$ & 98.6 & 96.8 & 1.8 \\
\hline $1: 00: 00$ & 100.7 & 100.0 & 0.7 \\
\hline
\end{tabular}


Figure 2-7 Compounds studied/of interest for future work (Chem. Draw)

This figure represents some of the compounds studied during the research (i.e. acetaminophen, aspirin). Also, other compounds and combinations of these compounds will be studied during future research. These potential compounds include ibuprofen, naproxen and caffeine.

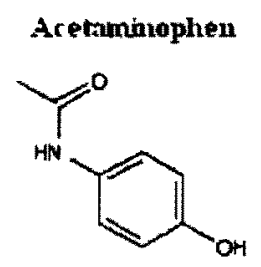<smiles>CC(C)Cc1ccc(C(C)C(=O)O)cc1</smiles><smiles>COc1ccc2cc([C@@H](C)C(=O)O)ccc2c1</smiles>

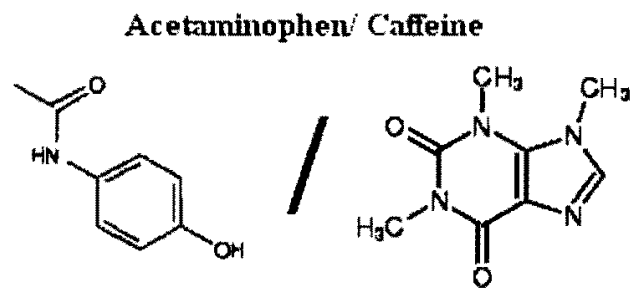

Acetaminophen Aspirin Caffeine<smiles>CC(=O)Nc1ccc(O)cc1</smiles><smiles>CC(=O)Oc1ccccc1C(=O)O</smiles><smiles>Cn1c(=O)c2ncn(C)c2n(C)c1=O</smiles> 


\subsection{Chapter summary}

In situ infrared spectroscopy was found to be a viable alternative for measuring dissolution profiles of pharmaceutical tablets. The IR system was found to be impressive in its capability of measuring very low concentrations. The limit of detection was found to be $0.02 \mathrm{mg} / \mathrm{ml}$ per IUPAC calculations. The IR system was able to distinguish separate components of a multiple component system without requiring manual sampling. This versatility is demonstrated by observing the simultaneous dissolution of acetaminophen and salicylic acid tablets. In contrast, UV-Vis spectroscopy may require mathematical corrections to resolve the two active drugs. Furthermore, HPLC must be used which requires laborious manual sampling or purchase of a complicated automated instrument setup of dissolution with HPLC analysis. The in situ ATR-IR method has been validated by comparing dissolution profiles using UVVis and HPLC methods. In addition, an accuracy of $\pm 2 \%$ between IR and HPLC for the salicylic acid results was established. With the current configuration of the IR instrument, this analysis is limited by the sensitivity and wavelength range of the in situ fiber optic probe. However, since this chapter successfully demonstrates the versatility of this novel application of ATR-IR spectroscopy, it is clear that the method has excellent potential to be improved by modification of the IR instrument and selection of more sensitive probes. 


\section{$3 \quad$ Kinetics of drug hydrolysis of common over-the-counter (OTC) drugs via ATR-IR spectroscopy}

This chapter focuses on describing the hydrolysis of acetylsalicylic acid (aspirin) under acidic conditions. The hydrolysis of aspirin was tested and monitored using ATR-IR spectroscopy. In the presence of an acidic medium, acetylsalicylic acid undergoes hydrolysis ${ }^{41}$ to form salicylic acid by the following acid-catalyzed mechanism:<smiles>CC(=O)Oc1ccccc1C(=O)Oc1ccccc1C(=O)O</smiles><smiles>C[C@@H](O)Oc1ccccc1C(=O)O</smiles><smiles>CC(=O)O[18O]C=C[C@H](O)[C@@H](O)C(=O)Oc1ccccc1O</smiles>

\footnotetext{
${ }^{41}$ Choudhury, S.; Mitra, A.K. Kinetics of aspirin hydrolysis and stabilization in the presence of 2hydroxypropyl-B-cyclodextrin. Pharmaceutical Research 1993, 10 (1), 156-159.
} 


\subsection{Background and history of Aspirin}

Aspirin is unique in its history and has many important roles in drug therapy. Aspirin is known to decrease pain. It fights the pain and inflammation by blocking the action of an enzyme called cyclooxygenase which inhibits the formation of prostaglandins. ${ }^{42}$ Prostaglandins are chemicals that signal an injury and trigger pain. Moreover, aspirin is mostly known for its ability to reduce strokes. Since aspirin inhibits the formation prostaglandins this results in the inhibition of blood clots which could cause a heart attack or stroke.

Figure 3-1 Structures of acetylsalicylic acid (aspirin)

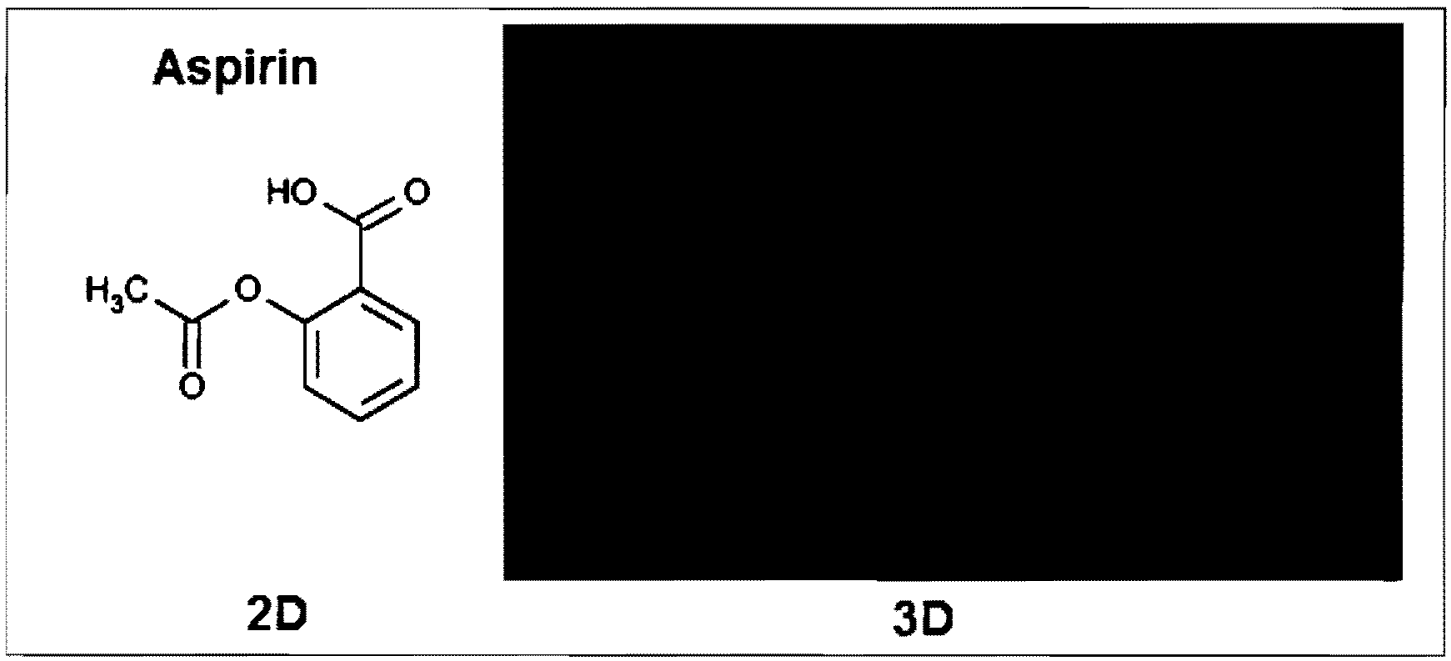

\footnotetext{
${ }^{42}$ Ito, Seiji; Okuda, Emiko; Minami, Toshiaki. Central and peripheral roles of prostaglandins in pain and their interactions with novel neuropeptides nociceptin and nocistatin. Neuroscience Research 2001, 41(4), $299-332$.
} 
The first synthesis of aspirin is credited to Gerhardt ${ }^{43}$ in 1853 . Gerhardt was investigating mixed organic acid anhydrides. Felix Hoffman (1868-1946) used acetic anhydride ${ }^{44}$ for aspirin's preparation. Felix Hoffmann synthesized aspirin in the laboratories of Farbenfabriken Bayer, Elberfeld, Germany in $1897 .^{45}$ Legend has it that Hoffman wanted to help his sick father who was suffering from rheumatism and was no longer able to tolerate sodium salicylate, then widely used for his father's arthritis disease. Salicylic acid, however, has several bad flaws. Its bad taste, stomach irritation and other side effects spurred researchers to look for other derivatives. The intent was to keep salicylic's efficacy without the disadvantages it posed. Acetylation of the hydroxyl group was one of the logical modifications. This logical thinking eventually led to the synthesis and discovery of aspirin. Refer to Figure 3-2 below for one of the synthesis pathways that were investigated back in the late 1800 's.

Figure 3-2 Synthesis of aspirin-late 1800's (Chem. Draw)

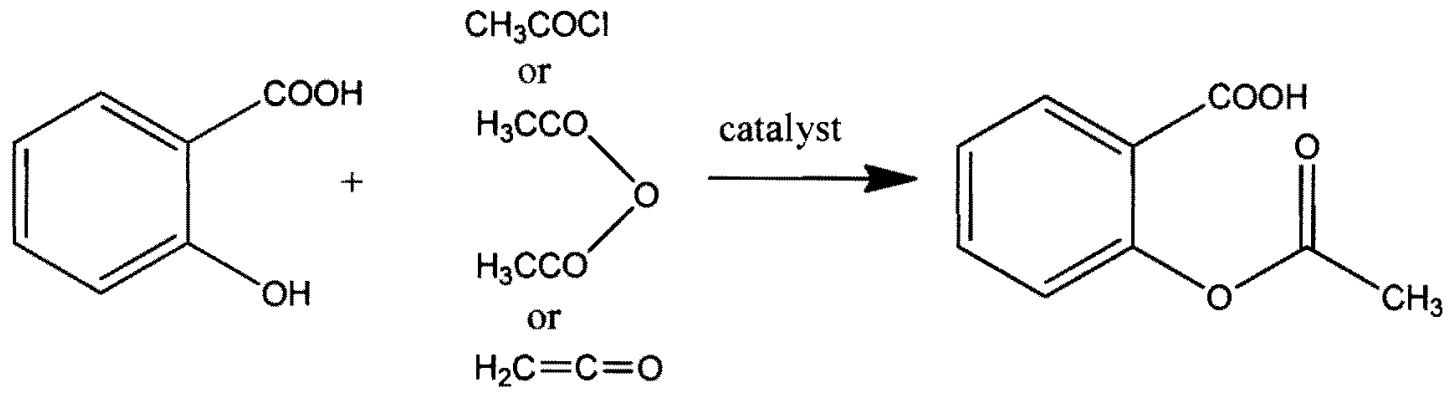

${ }^{43}$ C. Gerhardt, Annalen der Chemie, 67, 149(1853)

${ }^{44}$ F. Hoffmann, U.S. Patent C44077 of February $27,1900$.

${ }^{45}$ Analytical Profiles of Drug Substances, 8 Aspirin, Klaus Florey, (1979). 


\subsection{Experimental section}

\subsubsection{Chemical and materials}

Acetylsalicylic acid (aspirin) reference material (batch no. 016K0131) was purchased from Sigma-Aldrich. This same material was used "as is" for the powdered aspirin. Salicylic acid reference material (batch no. 04708HE) was purchased from Sigma-Aldrich. Acetylsalicylic acid $325 \mathrm{mg}$ tablets (Bayer batch no. $219050 \mathrm{~N}$ ) were purchased from a local pharmacy. Methanol, acetone and acetonitrile (HPLC grades) were purchased from Pharmaco-Aaper. Sodium chloride crystals (batch no. J39602), used to prepare the Simulated Gastric Fluid (SGF) reagent, was purchased from Mallinckrodt Chemicals. Conc. hydrochloric acid (batch no. H44032), used to prepare the SGF reagent, was purchased from J.T. Baker. All solutions were prepared using water treated by a Milli-Q Millipore purification system. All purified

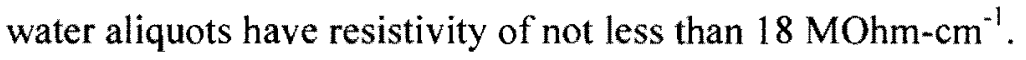


Figure 3-3 Chemical structures studied (Chem. Draw)

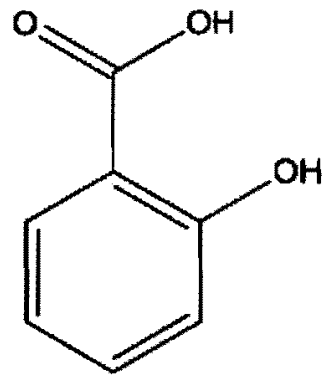

Salicylic Acid

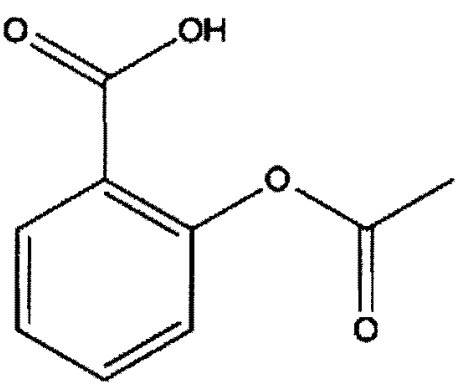

Acetylsalicylic acid (aspirin) 


\subsubsection{Instrumentation}

Samples were tested using Mettler Toledo's iC10 FT-IR system using a fiber optic probe equipped with a $1 \mathrm{~mm}$ diamond coated ATR probe. The IR system was operated by Mettler Toledo's iC IR version 3.0 or 4.0 software. The $\mathrm{pH}$ determinations were carried out using a Symphony $\mathrm{pH}$ meter (model no. SB70P). All dissolution experiments were tested in Mettler Toledo EasyMax 102 apparatus using iC Control 4.1 software. All analytical weight measurements were carried out using Mettler Toledo's DeltaRange and AG204 DeltaRange scale.

\subsubsection{Buffered solutions}

The Simulated Gastric Fluid ("SGF"), pH 1.2, was prepared with sodium chloride and aqueous hydrochloric acid in accordance with the USP. ${ }^{46}$ The detailed procedure for preparing this test solution is shown below

\section{Hydrochloric Acid Solution (0.2M):}

The volumes shown in the table are for $200 \mathrm{~mL}$ of buffer solution. Note: Where water is specified for solution or dilution of test substances in $\mathrm{pH}$ determinations, use carbon dioxidefree water.

- Sodium Hydroxide (0.2 M) - Dissolve $4.0 \mathrm{~g}$ of sodium hydroxide in water to make 100 $\mathrm{mL}$.

- Potassium Chloride $(0.2 \mathrm{M})$ - Dissolve $14.91 \mathrm{~g}$ of potassium chloride $(\mathrm{KCl})$ in water, and dilute with water to $1000 \mathrm{~mL}$.

\footnotetext{
${ }^{46}$ US Pharmacopoeia \& National Formulary-Gastric Fluid, Simulated, USP [Online], http:/www.usp.org/ (accessed May 1, 2011).
} 
Table 3-1

Hydrochloric acid buffer preparations as per United States Pharmacopeia (USP)

Composition of Standard Buffer Solutions

Hydrochoric Acid Euffer

Place $50 \mathrm{~mL}$ of the potassium chloride solution in a $200 \mathrm{~mL}$. volumetric flask, add the specified volume of the hydrochloric acid solution, then

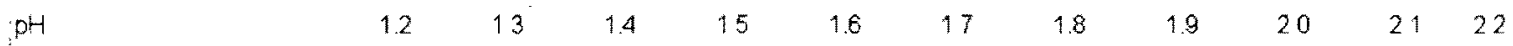

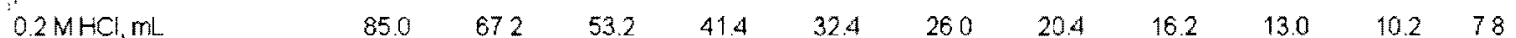

\subsubsection{Hydrolysis Experiments}

\section{HYDROLYSIS USING ASPIRIN TABLETS:}

Aspirin tablets: $325 \mathrm{mg}$ acetylsalicylic acid tablets were tested in pH 1.2 simulated gastric fluid solutions. Dissolutions were conducted using vessel volumes of $100 \mathrm{~mL}$ and at $37{ }^{\circ} \mathrm{C}$. All dissolutions were conducted using an agitation speed of $100 \mathrm{rpm}$. The dissolution run times were set for 1.5 hours.

\section{HYDROLYSIS USING ASPIRIN POWDER:}

Aspirin powder: acetylsalicylic acid powder samples were tested in $\mathrm{pH} 1.2$ simulated gastric fluid solutions. Dissolutions were conducted using vessel volumes of $100 \mathrm{~mL}$ and at $37^{\circ} \mathrm{C}$. All dissolutions were conducted using an agitation speed of $100 \mathrm{rpm}$. The dissolution run times were set for 1.5 hours.

\subsubsection{ATR-IR analysis}

The ReactIR ${ }^{\mathrm{TM}}$ iC10 FTIR instrument is composed of an MCT detector (liquid nitrogen cooled) and the FiberConduit ${ }^{\mathrm{TM}}$. The FiberConduit ${ }^{\mathrm{TM}}$ is comprised of flexible IR transparent silver chloride/silver bromide optical fibers. The fiber optic probe interface $(\mathrm{AgX} 9.5 \mathrm{~mm} \times$ $1.5 \mathrm{~m}$ Fiber (Silver Halide)) contains a diamond tip-DiComp ATR crystal. The resolution was 
set to 8 wavenumbers. The optical range used by the system is: $1900 \mathrm{~cm}^{-1}$ to $650 \mathrm{~cm}^{-1}$. The gain adjustment was set to normal $(1 \mathrm{x})$ and the apodization method was set to Happ-Genzel. ${ }^{47}$ The system uses compressed air (house air, filtered and dehumified) to purge the optics.

\section{HYDROLYSIS USING ASPIRIN TABLETS:}

For the hydrolysis of aspirin tablet experiments, data treatment was carried out using the following methodology: The data were first subjected to baseline correction. An absorption band at $1202 \mathrm{~cm}^{-1}\left(1202-1198 \mathrm{~cm}^{-1}\right)$ was selected for acetylsalicylic acid. The peak area was calculated using a two-point baseline correction set at $1180 \mathrm{~cm}^{-1}$ and $1203 \mathrm{~cm}^{-1}$. The ATR-IR system was configured to collect spectra every two minutes.

For this experiment, data processing is critical to obtain well-resolved spectra. Here is how the data is processed. First, the peak of interest was identified $\left(1202 \mathrm{~cm}^{-1}\right)$ and the area was calculated using a two-point baseline correction. Next, a trend analysis was performed using the iCIR software on this peak. This provided a plot of absorbance vs. time. This plot was subjected to smoothing using a 25 point smoothing parameter. After smoothing, and while still in the trend analysis, the baseline correct routine was performed. Then, the data were exported to Excel. Further data processing in Excel involved the subtraction of solvent spectra from reaction spectra. To perform this, a $30 \mathrm{~min}$ set of spectra at the beginning of the experiment, before aspirin pill was added, was selected. These spectra were subtracted from the spectra taken after the pill was dropped to give the final set of data. Within the final set of data, the area of the $1202 \mathrm{~cm}^{-1}$ peak was calculated at each time point. Then plot of area vs.

\footnotetext{
${ }^{47}$ Application Note: Effect of resolution to vapor phase spectrum of $\mathrm{HCl}$ in the Infrared Spectroscopy, Shimadzu Co., http://www2.shimadzu.com/applications/ftir/Appl_FTIR_HCl-vapor-phase_06D_en.pdf, accessed 25 May 2011.
} 
time were performed to give rate information. In addition, using a calibration curve, peak areas were converted to concentration to provide concentration vs. time plots.

An absorption band at $1086 \mathrm{~cm}^{-1}\left(1080-1090 \mathrm{~cm}^{-1}\right)$ was selected for salicylic acid. The peak area was calculated using a two-point baseline correction set at $1078 \mathrm{~cm}^{-1}$ and $1101 \mathrm{~cm}^{-1}$. The ATR-IR system was configured to collect spectra every two minutes.

Preparation of salicylic acid standards: $325 \mathrm{mg}$ of standard salicylic acid was added to a 100 $\mathrm{mL}$ volumetric flask and $50 \mathrm{~mL}$ of SGF solution was added and the flask was sonicated for 1 min to ensure all solids were dissolved. Finally, the solution was diluted to $100 \mathrm{~mL}$ using SGF.

Preparation of acetylsalicyclic acid standards (in this case, there are competing concerns about the lower solubility of the compound and the need to limit the amount of hydrolysis of the compound): To decrease time, the IR was set-up and background spectra were taken prior to preparing the standard solution. Acetylsalicyclic acid $(325 \mathrm{mg})$ was added to a $100 \mathrm{~mL}$ volumetric followed by $75 \mathrm{~mL}$ of SGF. The flask was sonicated for $10-15$ to effect complete dissolution of the solids, diluted to $100 \mathrm{~mL}$ with SGF.

\section{HYDROLYSIS USING ASPIRIN POWDER:}

For the hydrolysis of aspirin powder experiments, data treatment was carried out using the procedure described above. An absorption band at $1202 \mathrm{~cm}^{-1}\left(1202-1198 \mathrm{~cm}^{-1}\right)$ was selected for acetylsalicylic acid. The peak area was calculated using a two-point baseline correction set at $1180 \mathrm{~cm}^{-1}$ and $1203 \mathrm{~cm}^{-1}$. The ATR-IR system was configured to collect spectra every two minutes. 
An absorption band at $1086 \mathrm{~cm}^{-1}\left(1080-1090 \mathrm{~cm}^{-1}\right)$ was selected for salicylic acid. The peak area was calculated using a two-point baseline correction set at $1078 \mathrm{~cm}^{-1}$ and $1101 \mathrm{~cm}^{-1}$. The ATR-IR system was configured to collect spectra every two minutes.

For all ATR-IR experiments, 256 scans were collected and co-added for each spectral point. On average, every spectral point, took about two-minutes to complete. For all testing, the calculated peak response was subjected to mathematical smoothing using the $\mathrm{iC}_{10}$ software. The data were compared to reference standards measurements collected prior to start of the linearity and dissolution experiments.

Preparation of salicylic acid standards: $325 \mathrm{mg}$ of standard salicylic acid was added to a 100 $\mathrm{mL}$ volumetric flask and $50 \mathrm{~mL}$ of SGF solution was added and the flask was sonicated for 1 min to ensure all solids were dissolved. Finally, the solution was diluted to $100 \mathrm{~mL}$ using SGF.

Preparation of acetylsalicyclic acid standards (in this case, there are competing concerns about the lower solubility of the compound and the need to limit the amount of hydrolysis of the compound): To decrease the preparation time, the IR was set-up and background spectra were taken prior to preparing the standard solution. Acetylsalicyclic acid (325 mg) was added to a $100 \mathrm{~mL}$ volumetric followed by $75 \mathrm{~mL}$ of SGF. The flask was sonicated for $10-15$ to effect complete dissolution of the solids, diluted to $100 \mathrm{~mL}$ with SGF.

All dissolution experiments were allowed to equilibrate in the buffer for at least half an hour prior to the start of a dissolution experiment. Dissolution data were collected and plots of percent $(\%)$ dissolved vs. time were made. 


\subsection{Results and discussion}

\subsubsection{Hydrolysis of aspirin to salicylic acid in test tubes}

Prior to starting the hydrolysis experiments, a qualitative measurement was investigated for determining the presence of salicylic acid in solution. As discussed earlier, acetylsalicylic acid hydrolyzes to salicylic acid in the presence of an acidic medium. Ferric chloride was found to be a good qualitative test for salicylic acid. Ferric chloride, or iron (III) chloride, will not react with aspirin. It will, however, react with salicylic acid, which is used to synthesize aspirin and is also the hydrolysis product of aspirin. Moreover, the formation of an iron-phenol complex will occur. ${ }^{48}$ Thus, adding an aqueous ferric chloride solution to a sample of aspirin is a good way to see if there is any salicylic acid present and a purple color is an indication of salicylic acid. A sample of pure aspirin should not exhibit any color change. The ferric chloride color test is shown in Figure 3-4.

\footnotetext{
${ }^{48}$ Cha, K.; Park, K. Determination of iron (III) with salicylic acid by fluorescence quenching method. Talanta $1998,46(6), 1567-1571$.
} 
Seton Hall University

Figure 3-4 Ferric chloride color test- control samples
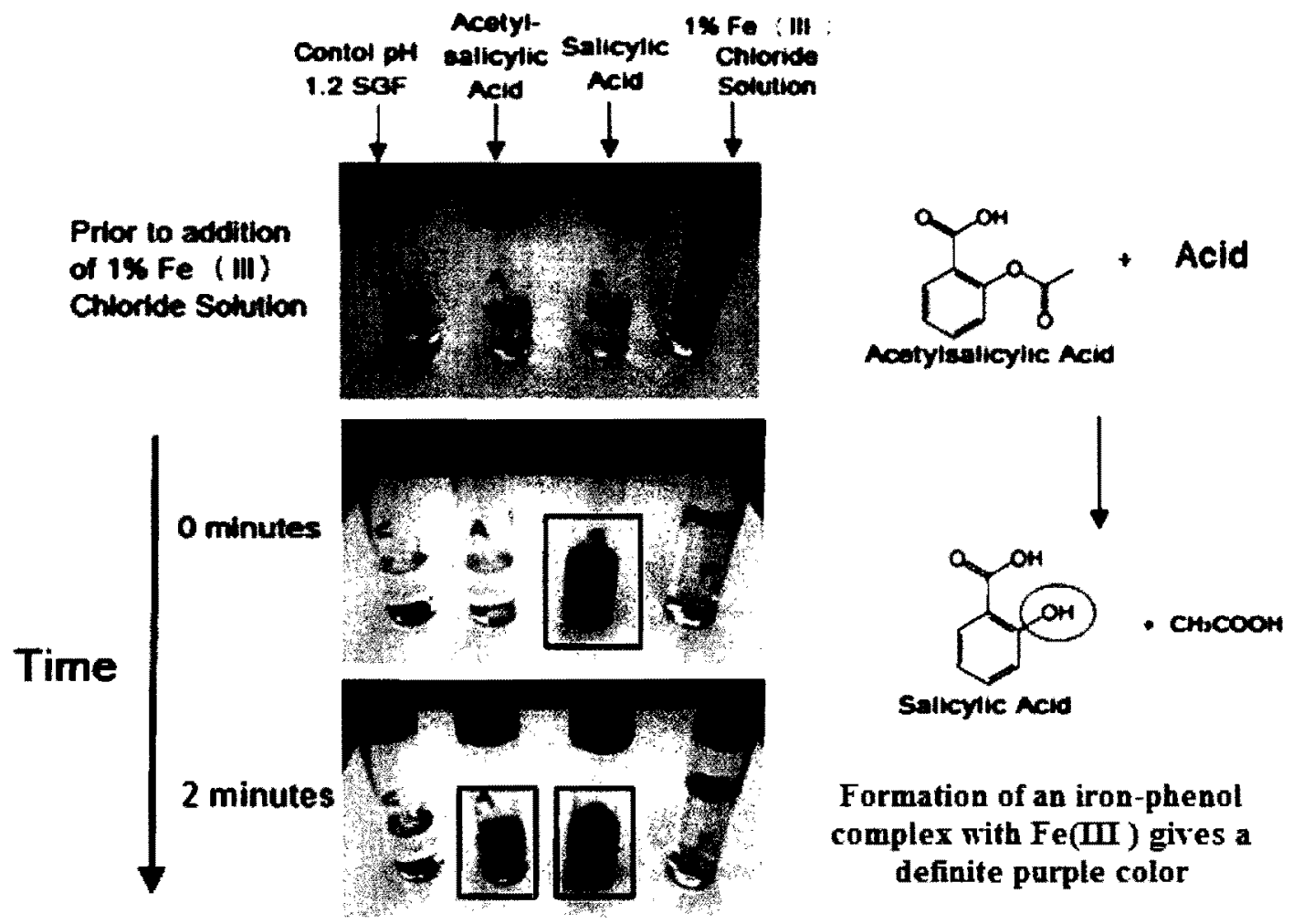

Formation of an iron-phenol complex with $\mathrm{Fe}(\mathrm{III})$ gives a definite purple color 
Table 3-2 Ferric chloride color test concentrations

\begin{tabular}{|c|c|c|}
\hline Experiment number & Weight of Aspirin (mg) & Weight of Salicylic Acid (mg) \\
\hline 1 & 32.1 & 33.4 \\
\hline 2 & 33.9 & 33.7 \\
\hline 3 & 31.7 & 35.1 \\
\hline Average weight $(\mathrm{mg})$ & 32.6 & 34.1 \\
\hline Volume of flask $(\mathbf{s})(\mathrm{ml})$ & 10.0 & 10.0 \\
\hline Average Concentration $(\mathrm{mg} / \mathrm{ml})$ & 3.3 & 3.4 \\
\hline
\end{tabular}

Table 3-3 Ferric chloride color test observations [added $2 \mathrm{mg} / \mathrm{ml} \mathrm{Fe}$ (III) solution]

\begin{tabular}{|c|c|c|c|}
\hline Time (min) & CONTROL Sample (SGF) & $\begin{array}{c}\text { Acetylsalicylic acid } \\
\text { Sample }\end{array}$ & Salicylic Acid Sample \\
\hline $0 \mathrm{~min}$ & Clear (no color change) & Clear (no color change) & Dark purple \\
\hline $1 \mathrm{~min}$ & Clear (no color change) & Clear (no color change) & Dark purple \\
\hline $2 \mathrm{~min}$ & Clear (no color change) & Light purple & Dark purple \\
\hline $5 \mathrm{~min}$ & Clear (no color change) & Dark purple & Dark purple \\
\hline
\end{tabular}




\subsubsection{Hydrolysis of aspirin to salicylic acid using aspirin tablet as the starting ingredient}

The hydrolysis of acetylsalicylic acid (aspirin) tablets was carried out in pH 1.2 SGF solutions. The solutions were preheated to body temperature, $37{ }^{\circ} \mathrm{C}$, and the ATR-IR fiber optic probe collected baseline spectra (blank medium) for the first 30 minutes. A single 325 $\mathrm{mg}$ Bayer aspirin tablet was dropped into the vessel containing $100 \mathrm{~mL}$ of $\mathrm{pH} 1.2$ solution.

Standard spectra of pure acetylsalicylic acid (aspirin) and salicylic acid were collected prior to running the dissolutions. These standards were used to identify the unique peaks of interests for these active drugs and for calculating absorbance intensities to concentration. As shown in Figure 3-5, aspirin has a unique band at $1202 \mathrm{~cm}^{-1}$ while salicylic acid has one at $1086 \mathrm{~cm}^{-1}$. Dissolution plots for aspirin and salicylic acid hydrosylate were made. Refer to Figure 3-6 which plots the concentration of acetylsalicylic acid vs. time and salicylic acid hydrosylate vs. time. This plot shows the formation and elimination of acetylsalicylic acid. Moreover, the acetylsalicylic acid hydrolyzed completely to form salicylic acid as shown in the plot. The presence of salicylic acid is detected by the IR system and plotted on a secondary axis as shown in Figure 3-6. In addition, the ferric chloride color test was used to visually inspect the presence or absence of salicylic acid. The ferric chloride solution was added to the aspirin tablet dissolution vessel after sixty minutes. The solution within the vessel quickly turned to a purple color as shown in Figure 3-7. 


\section{Figure 3-5 Waterfall plot of aspirin tablet dissolution}

This is a waterfall plot for the dissolution of $325 \mathrm{mg}$ aspirin tablet in SGF. As seen in the figure, there are two peaks of interest; one at $\sim 1086 \mathrm{~cm}^{-1}$ and one at $\sim 1205 \mathrm{~cm}^{-1}$. These represent salicylic acid and aspirin respectively. The purple color in the figure represents higher intensities while the blue green represent lower intensities or concentrations. As seen in the figure aspirin increases with time and then disappears while salicylic acid increases with time.

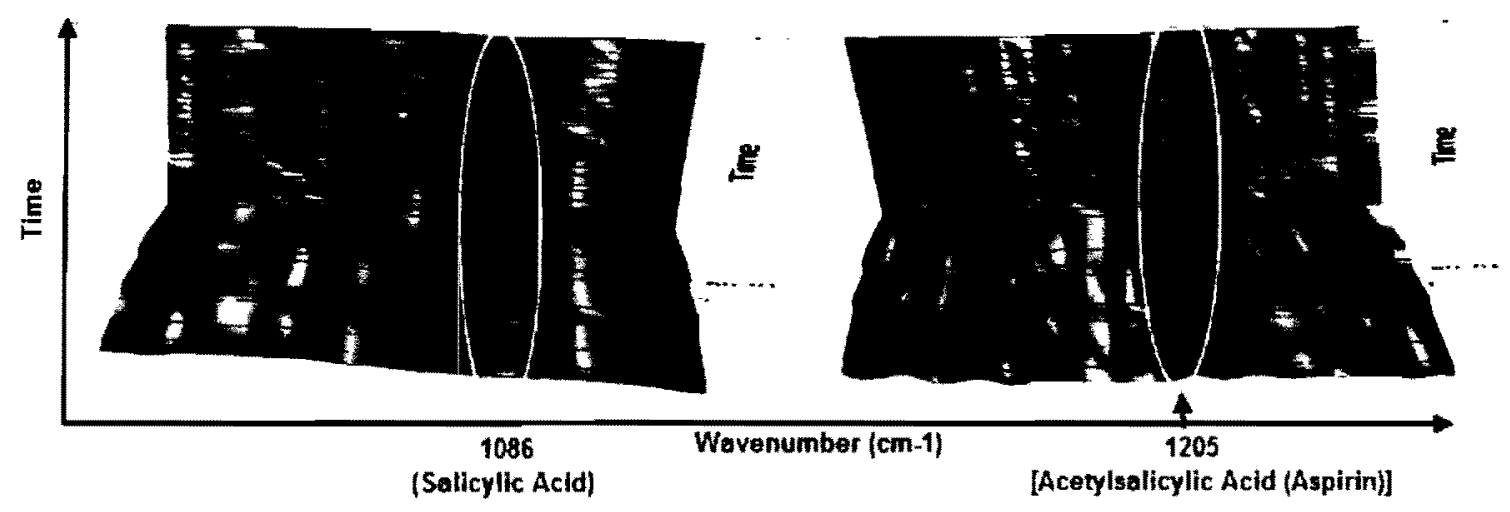


Figure 3-6

Hydrolysis of Aspirin Tablets (325 mg) in pH 1.2 SGF Solution ( $n=2)$

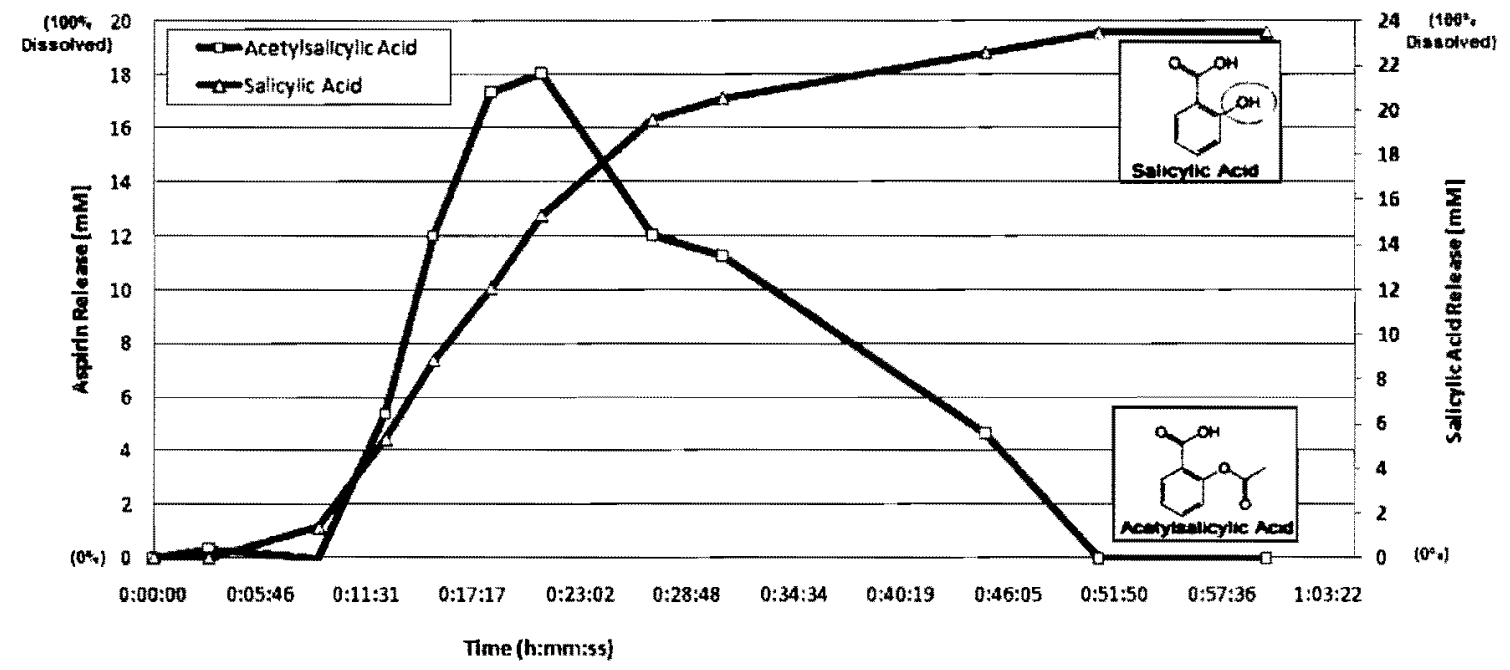

Figure 3-7 Ferric chloride color test for salicylic acid (tablet dissolution)

\section{Dissolution Vessel Ferric Chloride Test Results}

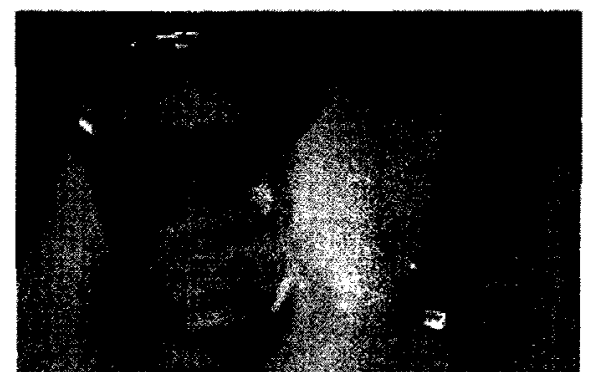

Time $=\mathbf{0}$ minutes

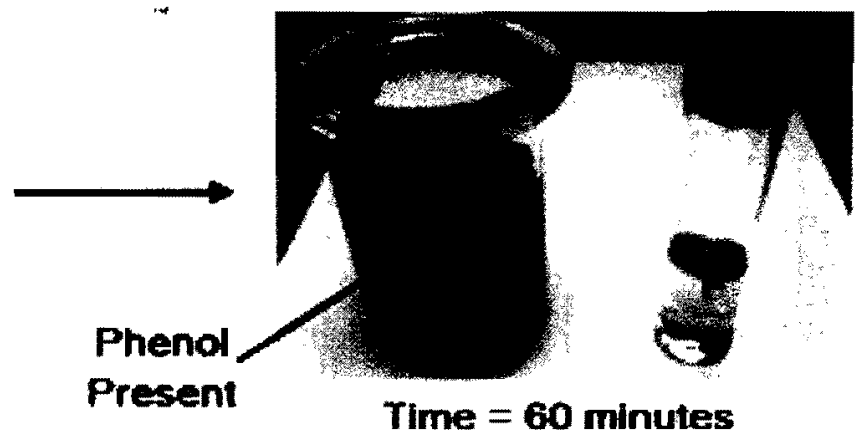




\subsubsection{Hydrolysis of aspirin to salicylic acid using aspirin powder as the starting ingredient}

It was equally important to understand the rate of hydrolysis of aspirin in powder form. Since the hydrolysis of aspirin in a tablet depends on the dissolution of aspirin into solution first, the rate of hydrolysis of pure aspirin powder should be relatively quicker because all particles are immediate exposed to the dissolving medium. In fact, the rate limiting step in this case would be the dissolution of the particles into the acidic medium. As a result, the hydrolysis of aspirin to salicylic acid was monitored and measured using pure aspirin powder. Known amounts, equivalent to the amount found in a tablet, were placed in the dissolution vessels containing an acid solution ( $\mathrm{pH} 1.2 \mathrm{SGF}$ ) and the dissolution profiles were collected using ATR-IR spectroscopy. Figure 3-8 shows the dissolution profiles of aspirin and salicylic acid using

aspirin powder. In addition, the figure clearly shows the formation and then elimination of acetylsalicylic acid in solution (black line) and the simultaneous formation of salicylic acid hydrosylate in solution (red line). 
Seton Hall University

Figure 3-8 Hydrolysis of acetylsalicylic acid powder to salicylic acid hydrosylate in $\mathrm{pH} 1.2$ solution

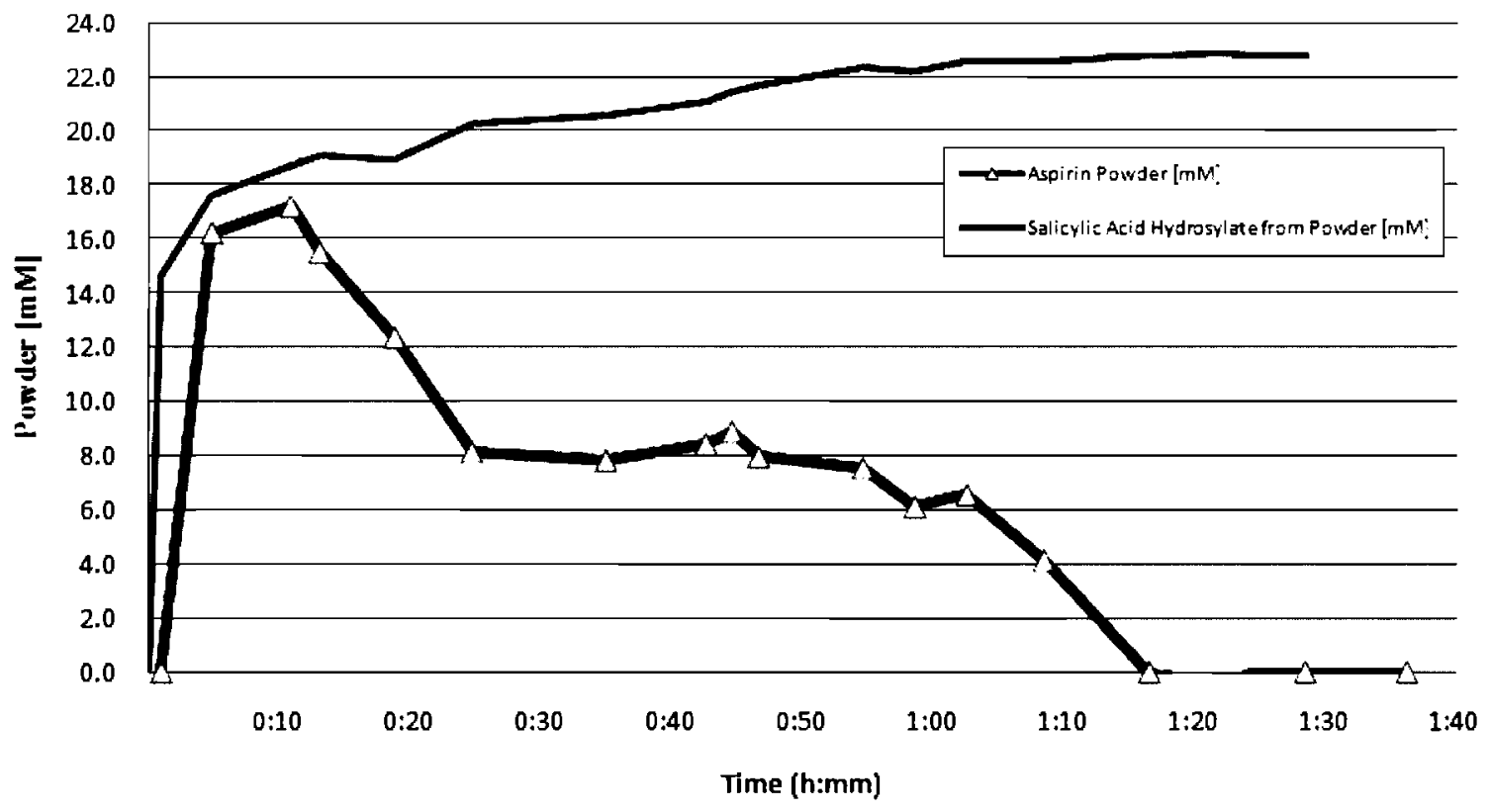




\subsubsection{Hydrolysis data: comparison between tablet and powder formulations}

Figure 3-9, compares the IR dissolution profiles of the salicylic acid component in powder and tablet form. As expected the rate of dissolution and hence hydrolysis of salicylic acid, is faster in the powder form. The tablet formulation has to undergo wetting, disintegration and deaggregation which take time. Similarly, Figure 3-10, compares the IR dissolution profiles of the aspirin component in powder and tablet form. As expected the rate of dissolution of aspirin, is faster in the powder form. For the tablets, in each case, there is an initial mechanical lag associate with the physical breakdown of the tablet. However, in the case of the experiments with the powders, there no mechanical lag and dissolution occurs immediately. However, with powdered acetylsalicylic acid, there is an interesting bimodal behavior. Initially, an immediate fast dissolution is observed and a secondary process is also observed at a slower rate as the dissolution occurs. Refer to Figure 3-8. The initial rapid dissolution likely occurs from particles that are well wetted in the initial exposure to the dissolution medium. However, aggregation of the powder was also observed suggesting that some of the acetylsalicylic acid particles were not wetted. The dissolution of these aggregates is associated with the slower dissolution process. In addition, the aspirin powder was used "as is" from the reagent container, therefore, the powder was inhomogeneous consisting of differ particle sizes with small and large crystal. It is likely that the larger particles and crystals contribute to the secondary dissolution process. However, that this process is not observed with salicylic acid (see Figure 3-9) is reasonable, first, because salicylic acid is more hydrophilic than acetylsalicylic acid, and, second, because salicylic acid dissolves at a faster rate, leaving less time for aggregation to occur. 
To develop an overall understanding of the dissolution/hydrolysis processes that occur with aspirin, a composite plot was prepared (Figure 3-11). In this plot, the dissolutions of aspirin powder and tablet and the hydrolysis of aspirin powder and tablet to form the salicylic acid hydrosylate are shown. The solid blue-line (aspirin powder) and the dotted red-line (aspirin tablet) compare the IR dissolution profiles of the aspirin component in powder and tablet form respectively. As expected, the rate of dissolution of aspirin is faster in the powder form. For the tablets, in each case, there is an initial mechanical lag associate with the physical breakdown of the tablet. However, in the case of the experiments with the powders, there no mechanical lag and dissolution occurs immediately. This plot also compares the release profiles of the salicylic acid hydrosylate in tablet (dotted green-line) and powder formulation (solid black line). Again, for the tablet, there is an initial mechanical lag associated with the physical breakdown of the tablet. Also, the hydrolysis of aspirin in the powder formulation appears to be quite rapid. Moreover, the plot shows this phenomenon occurring prior to the dissolution of the powder aspirin. Currently, the dissolution profiles were run at $100 \mathrm{rpm}$ with a collection rate of two-minutes. A higher agitation speed and faster acquisition time (i.e. every 1 minute) should synchronize the dissolution of the aspirin and then the release of the salicylic acid hydrosylate in the powder formulation. 
Figure 3-9 Release profile of Salicylic Acid ("SA") during hydrolysis in SGF (pH1.2): powder vs. tablet

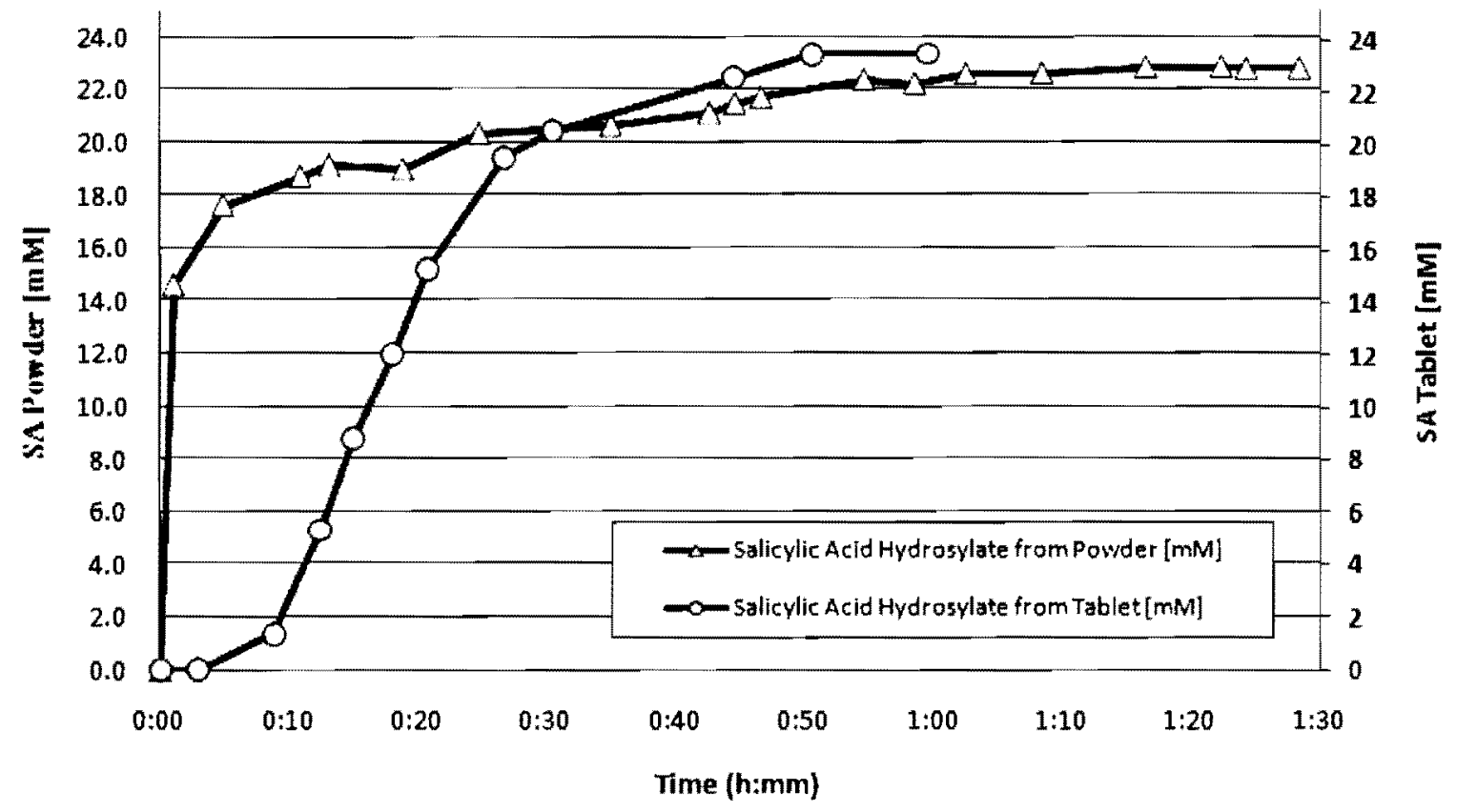


Figure 3-10 Release profile of acetylsalicylic acid (aspirin) during hydrolysis: powder vs. tablet

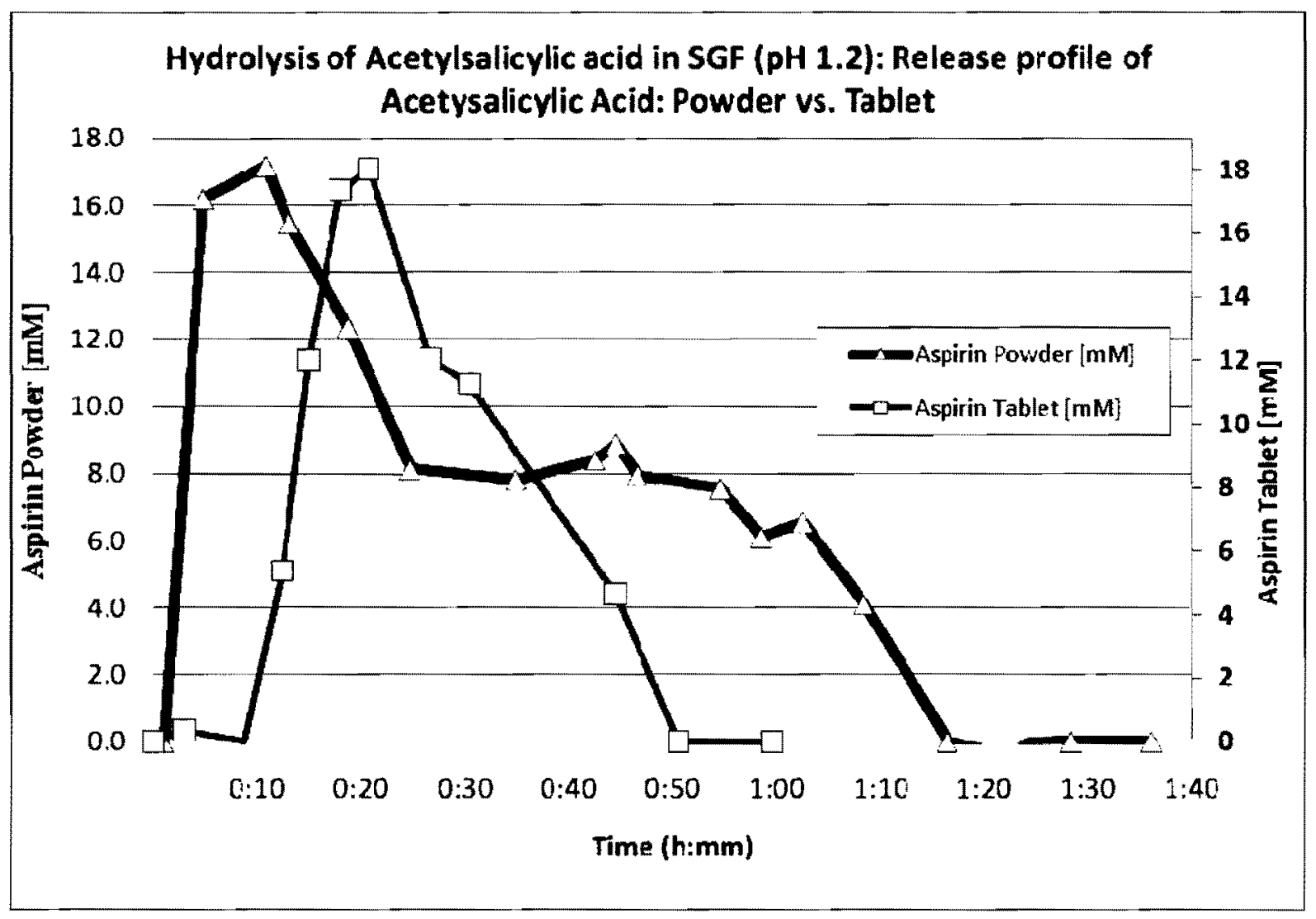


Figure 3-11 Release profile of acetylsalicylic acid (aspirin) and salicylic acid hydrosylate during hydrolysis: powder vs. tablet

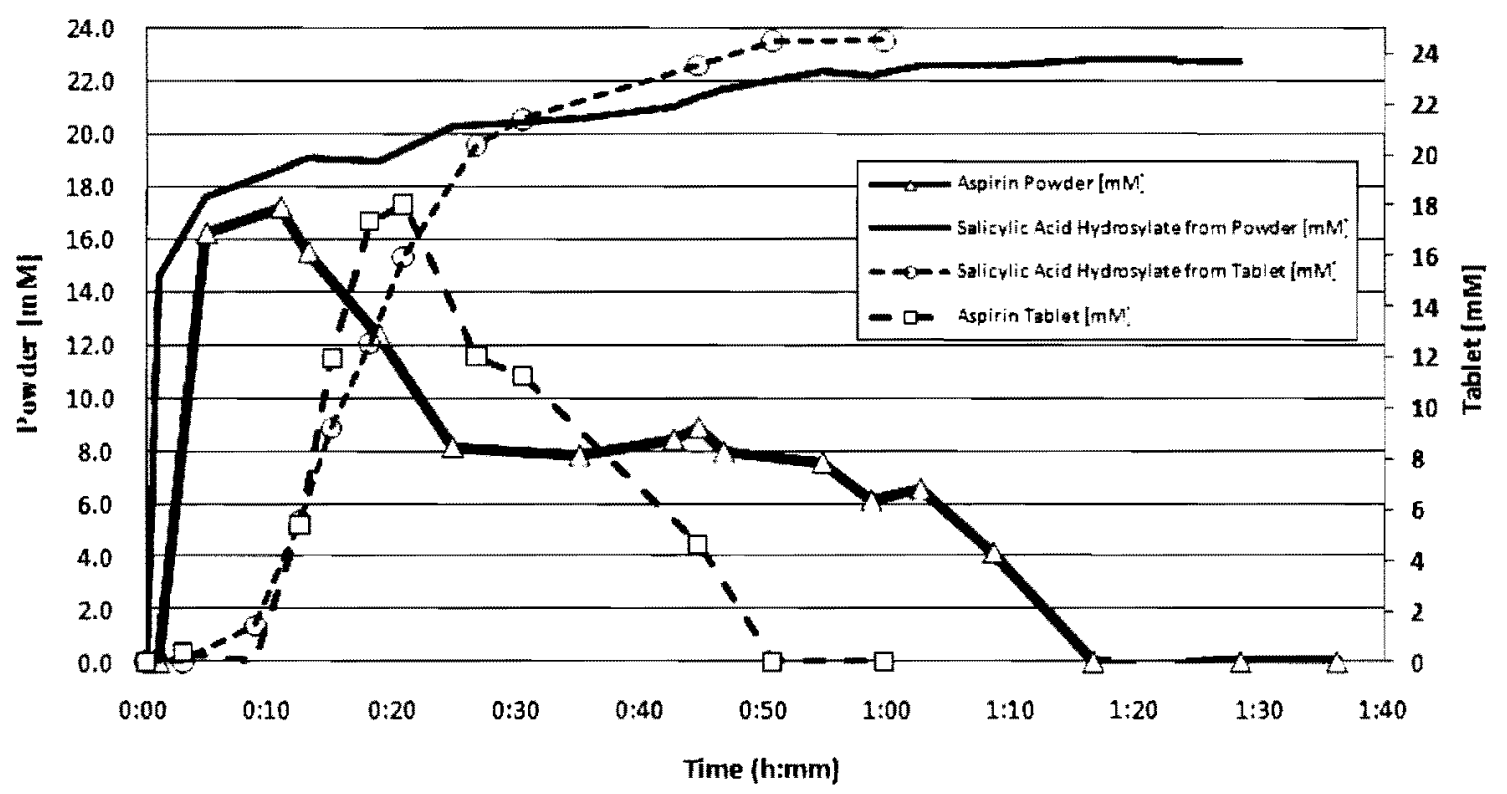




\subsubsection{Kinetic rates observed during hydrolysis experimentation}

The kinetic rates were also determined for the experiments discussed earlier. The rates were determined using the slopes of the dissolution plots. The rates were compared to literature research $^{49}$ and were found to be equivalent within experimental error. In fact, the literature data shows the rate of hydrolysis of aspirin under acid medium varies from $0.91 \mathrm{~min}^{-1}$ to 1.74 $\min ^{-1}$ depending on the $\mathrm{pH}$ and temperature of the solution. Refer to Table 3-4 for details. According to Habib and co-workers the hydrolysis rate of aspirin was found to be $0.91 \mathrm{~min}^{-1}$ at $\mathrm{pH} 1.0$ and $30^{\circ} \mathrm{C}$. Choudhury and co-workers reported the hydrolysis rate as $1.74 \mathrm{~min}^{-1}$ at $\mathrm{pH} 1.3$ and $40{ }^{\circ} \mathrm{C}$. If both literature studies are taken into account and a linear plot is constructed with rate vs. $\mathrm{pH} /$ temp, the expected rate of hydrolysis at conditions similar to this research should be $1.46 \mathrm{~min}^{-1}$. Furthermore, in this study, the hydrolysis of aspirin to salicylic acid, using OTC aspirin tablets in $\mathrm{pH} 1.2 \mathrm{SGF}$ and at $37^{\circ} \mathrm{C}$, was found to be $1.03 \mathrm{~min}^{-1}$. The hydrolysis of aspirin powder to salicylic acid was found to be $1.23 \mathrm{~min}^{-1}$.

\footnotetext{
${ }^{49}$ a) Habib, M.J.; Rogers, J.A. Kinetics of hydrolysis and stabilization of acetylsalicylic acid in liposome formulations. International Journal of Pharmaceutics 1998, 44, 235-241.b) Choudhury, S.; Mitra, A.K. Kinetics of aspirin hydrolysis and stabilization in the presence of 2-hydroxypropyl-B-cyclodextrin. Pharmaceutical Research 1993, 10 (1), 156-159.
} 
Table 3-4 Kinetic rates for hydrolysis of aspirin in acidic medium

\begin{tabular}{|l|l|}
\hline Description & Kinetics Rate $\left(1 / \mathrm{min}^{-1}\right)$ \\
\hline At pH 1.0 and $30^{\circ} \mathrm{C}$ (acetylsalicylic acid $=7.5 \mathrm{mM}$ ) Ref & - \\
\hline Rate of Hydrolysis & 0.91 \\
\hline
\end{tabular}

\begin{tabular}{|l|l|}
\hline At $\mathrm{pH} 1.3$ and $40^{\circ} \mathrm{C}$ (acetylsalicylic acid $=5.55 \mathrm{mM}$ ) Ref & -- \\
\hline Rate of Hydrolysis & 1.74 \\
\hline
\end{tabular}

\begin{tabular}{|l|l|}
\hline $\begin{array}{l}\text { At } \mathrm{pH} 1.2 \text { and } 37^{\circ} \mathrm{C} \text { (acetylsalicylic acid }=18 \mathrm{mM} \text { ) (this } \\
\text { work) }\end{array}$ & -- \\
\hline Rate of Dissolution (Tablet) & 1.62 \\
\hline Rate of Hydrolysis (Tablet) & 1.03 \\
\hline Rate of Dissolution (Powder) & 1.59 \\
\hline Rate of Hydrolysis (Powder) & 1.23 \\
\hline
\end{tabular}

The rates of the reactions were determined from the dissolution plots. Refer to Figure 3-10. In order to calculate the rates using the plot, the following procedure was implemented. First, a plot of \% dissolved vs. time is obtained. Next, a straight line is drawn over the area of the dissolution curve where dissolution starts to occur (middle area in this case). Furthermore, the slope of the curve is obtained and from this the rate of hydrolysis is reported. Refer to Figure 3-12 and Figure 3-13. 
Figure 3-12 Typical calculation plot of rate from dissolution

Below is a schematic diagram illustrating how to calculate the rate.

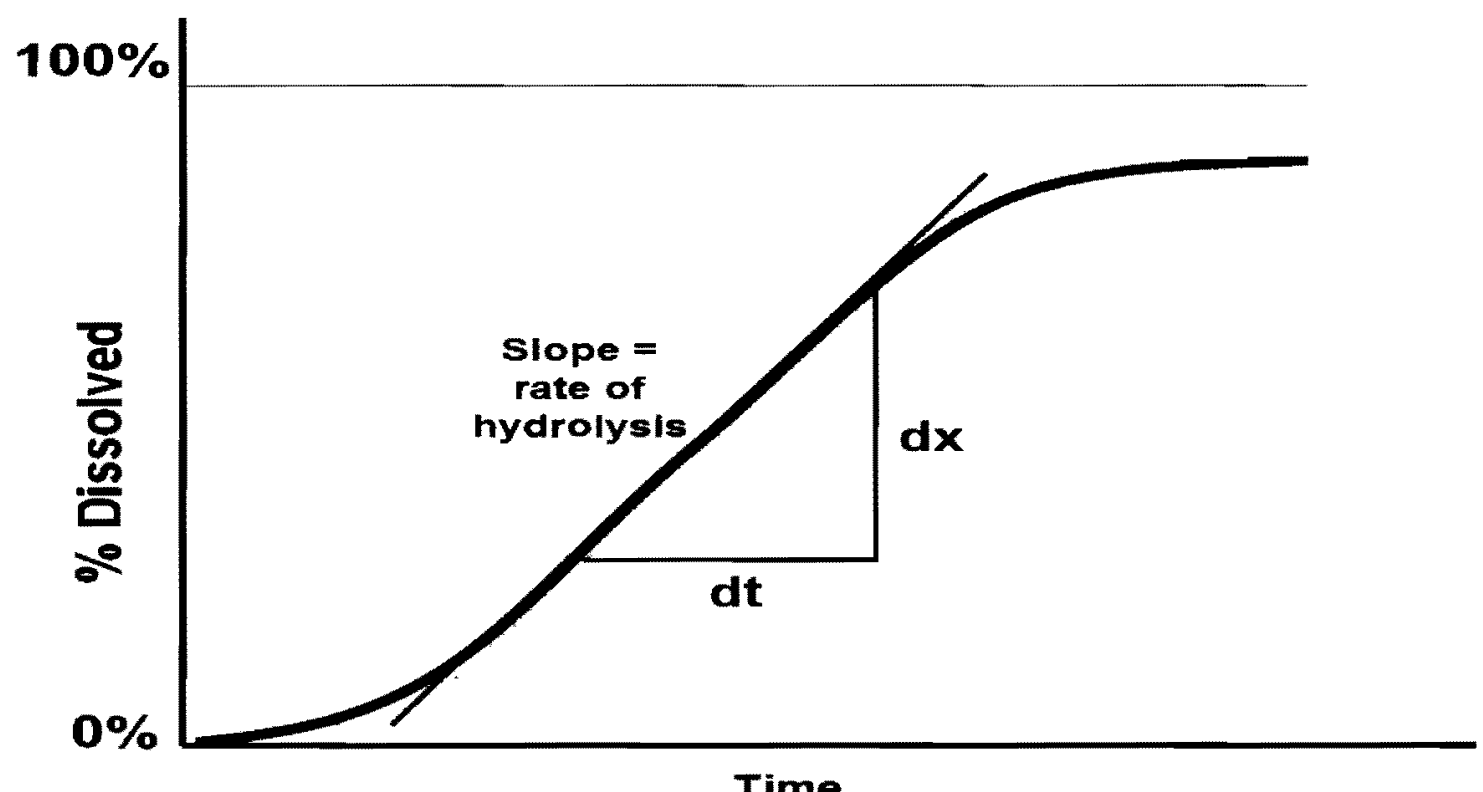

Figure 3-13 Rate of dissolution for Aspirin (based on slope of dissolution plot)

Hydrolysis of Aspirin

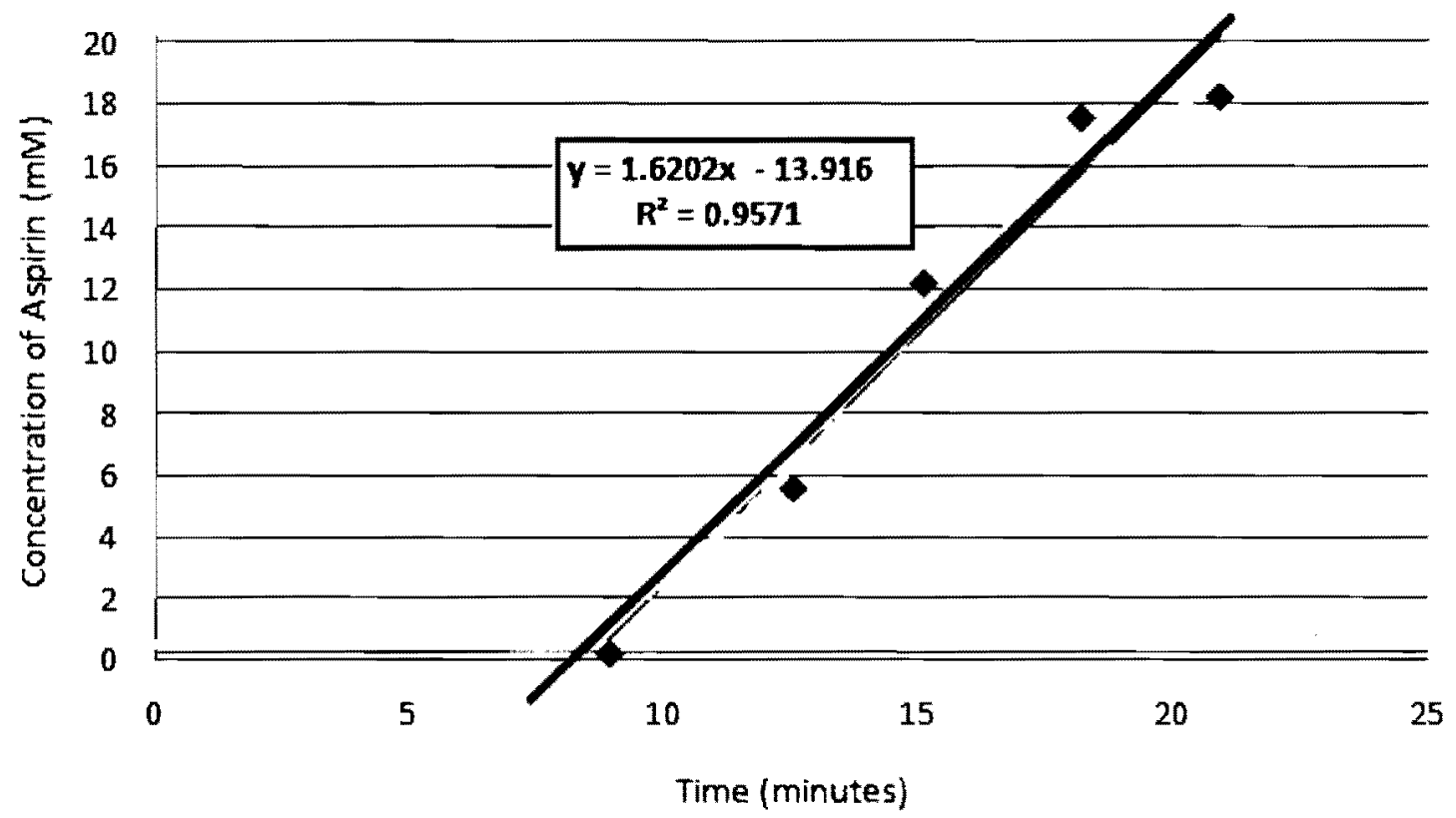

[86] 


\subsection{Chapter summary}

In this chapter, it was demonstrated that in-situ infrared spectroscopy is a viable technique for detecting and measuring the hydrolysis of acetylsalicylic acid (aspirin) in acidic media. The IR system was found to be impressive in its capability of measuring very low concentrations and was able to distinguish separate components of a multiple component hydrolysis system without requiring manual sampling. This versatility is demonstrated by observing the simultaneous dissolution and then conversion of the acetylsalicylic acid API to salicylic acid. In the tablet formulation, the rate of dissolution was found to be $1.62 \mathrm{~min}^{-1}$ based on the initial slope of the dissolution profile. Moreover, the hydrolysis rate was found to be $1.03 \mathrm{~min}^{-1}$ based on the salicylic acid formation and dissolution profiles. In the powder formulation, the rate of dissolution was $1.59 \mathrm{~min}^{-1}$. The rate of hydrolysis was found to be $1.23 \mathrm{~min}^{-1}$. As expected, this rate is slightly higher since tablets require an initial period of disintegration and deaggregation. Moreover, the rate of hydrolysis of the powder formulation is closer to Choudhury's finding. In the tablet experiment, the rate of hydrolysis of aspirin does not exactly match the rate of hydrolysis from the powder experiment. This could be explained by several reasons. First, there is competing dissolution within the vessel. The tablet has to undergo disintegration and dissolution prior to hydrolysis occurring. Second, the stirring rate was too slow. In the powder formulation, by the time the active drugs reaches the fiber optic probe, the hydrolysis has already begun. This is especially important in the powder form where there is not competing dissolution of tablet. Refer to Figure 3-9. The salicylic acid hydrosylate was observed before the acetylsalicylic acid from the powder. Third, the IR 
system was allowed to collect spectra every two minutes. This should be enhanced to collect every one minute in order to detect the hydrolysis quicker.

This research was possible because of the versatility of ATR-IR system. Specifically, the unique fingerprint region of the IR spectra gave detailed information about the release profile of the drug. Conventional methods, namely, in-situ UV-Vis, would only show the release profile of one component. However, peak deconvolution software can be used to analyze aspirin hydrolysis. Moreover, the hydrolysis and transformation of the drug from one form to another may be missed with conventional techniques. ATR-IR captures those minor details and displays them in the fingerprint region, as shown earlier in the results. The unique feature of this system is that it is able to detect several forms of the same drug. In other words, it was able to detect the formation and elimination of a pharmaceutical drug (aspirin) while detecting the formation of the hydrosylate of the aspirin (salicylic acid) in real-time. In addition, this system could be used to study other potential transformations. One example might be the dimerization of acetaminophen as mentioned earlier. The novelty with this particular system is its capability to see drug transformations in solutions while conducting the experiment insitu. Since this chapter successfully demonstrates the versatility of this novel application of ATR-IR spectroscopy, indicating that that the method has excellent potential for detailed studies of the dissolution and hydrolysis kinetics of pro-drug formulations and drugs which hydrolyze under acidic conditions simulating stomach conditions. 


\section{Instrument Development Proposal: "Solv-IR" as an In-Situ ATR-IR Instrument for Solvation Chemistry}

This chapter focuses on describing the design and potential application of the "Solv-IR" system. The Solv-IR system is a new system that combines dissolution technology with attenuated total reflectance-infrared spectroscopy (ATR-IR). A Major Research Instrumentation (MRI) grant was recently submitted with the National Science Foundation for funding of this project. The details, described below, give an overview of the new system as well describe the specific components of the proposed new system.

\subsection{Background}

Solvation, also sometimes called dissolution, is the process of attraction and association of molecules of a solvent with molecules or ions of a solute. As ions dissolve in a solvent they spread out and become surrounded by solvent molecules. By an IUPAC definition, solvation is an interaction of a solute with the solvent, which leads to stabilization of the solute species in the solution. ${ }^{50}$ Solvation is also a kinetic process with a thermodynamic conclusion. ${ }^{51}$ Most instrumentation used to investigate solvation focuses on the thermodynamic conclusion, i.e., the structure and concentration of the solvated species at equilibrium. Dynamic study of solvation either requires manual sampling or in situ UV-Vis detection which (in spite of excellent curve fitting software) has limited capability for multi-component analysis and for species that chemically transform. The goal is to construct a new instrument that allows

\footnotetext{
${ }^{50}$ IUPAC, Compendium of Chemical Terminology, 2nd ed. (the "Gold Book") (1997). Online corrected version: (2006-) "solvation".

${ }^{51}$ Anslyn, E. V.; Dougherty, D. A. Chapter 3: Solutions and Non-Covalent Binding Forces. Modern Physical Organic Chemistry; University Science, California, 2006.
} 
dynamic measurement of solvation of a chemical substance or chemical mixture with in situ infrared detection. Because of the large number of vibration modes available for many molecules, infrared spectroscopy is much more selective than UV-Vis. In addition, characteristics of the IR band can provide information about the mechanism of dissolution including hydrogen bonding interactions and aggregation of solute molecules. ${ }^{52}$ As discussed earlier, infrared spectroscopy using attenuated total reflectance (ATR) probes is quantitative, thus, providing precise measurement of solute concentrations. The proposed name for the instrument is Solv-IR which indicates that it uses infrared technology for investigation of solution chemistry. The target instrument will have several attractive features: a) $10^{-6} \mathrm{M}$ sensitivity, b) chemically robust; c) conventional IR spectral range; d) precise control of reaction vessel temperature; $f$ ) integrated stirring controls; $g$ ) $n=6$ solvation vessels to provide appropriate statistics; f) software for instrument control, data acquisition and processing. The Solv-IR will prove to be useful in the following scientific applications:

- Solvation of single component species such as homogeneous catalysts ${ }^{53}$ and substances with different crystalline morphologies. ${ }^{54}$

- Crystallization studies (the reverse of solvation!). ${ }^{55}$

\footnotetext{
${ }^{52}$ The Chemical Physics of Solvation, Part B, Spectroscopy of Solvation, R. R. Dogonadze, E. Kálmán, A. A. Kornyshev, J. Ulstrup, Eds., Elsevier, The Netherlands, 1986. See: Chapter 1: "Spectroscopic approaches to the study of ionic solvation," G. Kabisch, E. Kálmán; Chapter 2: "Interactions in and structures of ionic solutions and polyelectrolytes, Infrared Results," G. Zundel, J. Fritsch; Chapter 3: "Infrared Spectrscopic results on solvate structures in crystals," G. Zundel, J. Fritsch.

${ }^{53}$ Studies of catalyst dissolution, a) Sun, Y.K.; Le Blond, C. R.; Wang, J.; Blackmond, D. G.; Laquidara, J.; Sowa, J. R. Observation of a $[\mathrm{RuCl} 2(\mathrm{~S})-(-)$-tol-binap) $] 2 \cdot \mathrm{N}(\mathrm{C} 2 \mathrm{H} 5) 3-\mathrm{Catalyzed}$ Isomerization-Hydrogenation Network, Journal American Chemical Society 1995, 117, 12647. b) Shaw, J.; Chen, Y.S.; Fulton, J.; Linehan, J.; Gutowska, A.; Bitterwolf, T. Structural evolution of a recoverable rhodium hydrogenation catalyst. Journal of Organometallic Chemistry 2008, 693, 2111 -2118.

${ }^{54}$ a) Acquah, C.; Karunanithi, A. T.; Cagnetta, M.; Achenie, S. L. Linear models for prediction of ibuprofen crystal morphology based on hydrogen bonding propensities. Sub Fluid Phase Equilibria 2009, 277(1), 73-80.
} 
- Studies of chemical and mechanical parameters that effect solvation including temperature, agitation, solvent-type, $\mathrm{pH}$, shape and hardness of the solid. ${ }^{56}$

- *Studies of tablet formulations including chemical effects of co-ingredients. ${ }^{57}$

- *Monitoring of multi-component and controlled release formulations. ${ }^{58}$

- *Solvation rates for components that chemically transform on the solvation time scale..$^{59}$

- *Environmental chemistry for study of dispersants (emulsifier, surfactants) for treating chemical (e.g., petroleum) spills and other anthropomorphic events. ${ }^{60}$

- Kinetics of micelle formation. ${ }^{61}$

The above examples represent key applications of IR spectroscopy in the chemistry of solution; however, there are many more examples. ${ }^{62}$ Preliminary results have been obtained

b) Chieng, N; Rades, T; Aaltonen, J. An overview of recent studies on the analysis of pharmaceutical polymorphs. Journal of Pharmaceutical and Biomedical Analysis, In Press (2011).

${ }^{55}$ Halasz, I.; Biljan, I.; Novak, P.; Meštrović, E.; Plavec, J.; Mali, G.; Smrečki, V.; Vančik, H. Crossdimerization of nitrosobenzenes in solution and in solid state. Journal of Molecular Structure 2009, 918(1-3).

${ }^{56}$ Vitha, M.; Carr, P. W. The chemical interpretation and practice of linear solvation energy relationships in chromatography. Journal of Chromatography A 2006, 1126(1-2), 143-194.

${ }^{57}$ a) Van der Weerd, J.; Chan, K. L. A.; Kazarian, S. G. An innovative design of compaction cell for in situ FTIR imaging of tablet dissolution. Vibrational Spectroscopy 2004, 35(1-2), 9-13. b) Chan, K.L.A.; Elkhider, N.; Kazarian, S.G. Spectroscopic Imaging of Compacted Pharmaceutical Tablets. Chemical Engineering Research and Design 2005, 83(11), 1303-1310. c) Dressman, J. J.; Kramer, J. K., Eds. Pharmaceutical Dissolution Testing; Taylor and Francis: Florida, 2005. d) Hanson, R; Gray, V. Handbook of Dissolution Testing, 3rd ed., Dissolution Technologies: Delaware, 2004.

${ }^{58}$ Van der Weerd, J.; Kazarian, S.G.; Combined approach of FTIR imaging and conventional dissolution tests applied to drug release. Controlled Release 2004, 98, 295-305.

${ }^{59}$ Kokot, Z.; Burda, K. Simultaneous determination of salicylic acid and acetylsalicylic acid in aspirin delayedrelease tablet formulations by second-derivative UV spectrophotometry. Journal of Pharmaceutical and Biomedical Analysis 1998, 18, 871-875.

${ }^{60}$ a) Fingas, M. Oil Spill Dispersants: A Technical Summary. Oil Spill Science and Technology 2011, 435-582. b) Brandvik, J; Daling, S, Optimising oil spill dispersants as a function of oil type and weathering degree: a multivariate approach using partial least squares (PLS), Chemometrics and Intelligent Laboratory Systems 1998 , 42(1-2), 73-91. c) Regan, F.; Meaney, M.; Vos, J.G.; Maccraith, B.D.; Walsh, J.E. Determination of Pesticides in water using ATR-FTIR spectroscopy on PVC/chloroparaffin coatings. Analytical Chimica Acta 1996, 334, $85-92$.

${ }^{61}$ Ceraulo, L.; Dormond, E.; Mele, A.; Turco Liveri, V. FT-IR and nuclear overhauser enhancement study of the state of urea confined in AOT-reversed micelles. Colloids and Surfaces A: Physicochemical and Engineering Aspects 2003, 218(1-3), 255-264. 
for the above examples with an asterisk $\left({ }^{*}\right)$ using a single probe/vessel prototype. ${ }^{63}$ However, all of the above examples are important to chemists and the Solv-IR instrument will provide fundamental and practical insight into these processes.

\subsection{Broader impacts from the proposed activities}

The activities described in this project have several characteristics that are likely to have a broad impact on our nation's scientific infrastructure and perhaps provide economic opportunities. Thus, the broader impacts from the proposed activities are described here.

- First, there is substantial evidence that the proposed Solv-IR will be commercially viable. Investigation of solvation chemistry of single compounds or mixtures ensures that compounds are accurately delivered or dispersed at desired concentration and time. In the US alone there are at least nine companies that sell solvation units (basic: Hanson, Varian, Sotax, Logan, Distek; robotic: Zymark; UV-Vis monitoring: Pion, C-Technologies, Leap Technologies) and retail prices range from $\$ 20 \mathrm{~K}$ for basic units, $\$ 100 \mathrm{~K}$ for UV-Vis equipped units and $\$ 250 \mathrm{~K}$ for robotically controlled units. The total market capital of this industry is estimated to be $\$ 500 \mathrm{M}$ USD.

- Second, the Solv-IR is a potential driver to a field of research that is hungry for more information about the chemistry that is occurring inside the reaction vessel.

\footnotetext{
${ }^{62} \mathrm{~J}$. Workman Jr. Review of Near-Infrared and Infrared Spectroscopy. The Handbook of Organic Compounds; Academic Press: California, 2001; pp 79-129.

${ }^{63}$ Kassis, A.; Bhawtankar, V.M.; Sowa, J.R. Attenuated total reflection infrared spectroscopy (ATR-IR) as an in situ technique for dissolution studies. Journal of Pharmaceutical and Biomedical Analysis 2010, 53, 269-273.
} 


\subsection{Limit of detection (LOD) and limit of quantitation (LOQ) data}

Before the instrument design is discussed, an overview of limit of detection (LOD) and limit of quantitation (LOQ) will be made. ${ }^{64}$ The LOD and LOQ levels were calculated for the existing ATR-IR system coupled with different types of fiber optic probes. Three types of fiber optic probes were tested: DiComp probe, Flow-Cell probe and K4 probe. ${ }^{65}$ Differences between these probes will be discussed below.

As per the IUPAC, The limit of detection, expressed as a concentration or quantity, is derived from the smallest measure that can be detected with reasonable certainty for a given analytical procedure. The limit of detection is the lowest concentration of an analyte that the analytical process can reliably detect. ${ }^{66}$ The LOD level is calculated from the average of the blank, standard deviation of the blank and a confidence factor. ${ }^{67}$ The limit of quantification refers to the smallest concentration or the mass which can be quantitatively analyzed with reasonable reliability by a given procedure. There are several techniques available to calculate detection limits. The limit of detection is estimated as three times the standard deviation of the blank and the limit of quantitation is estimated as ten times the standard deviation of the blank. Of the three fiber optic probes mentioned earlier, the DiComp probe is the most useful to dissolution chemistry. The DiComp probe is diamond coated to protect the Zn-Se ATR crystal from attack by acidic medium. Since a lot of the dissolutions are carried out in acidic medium ( $\mathrm{pH} \mathrm{1-3),} \mathrm{the} \mathrm{ATR} \mathrm{crystal} \mathrm{needs} \mathrm{the} \mathrm{extra} \mathrm{coating} \mathrm{to} \mathrm{ensure} \mathrm{longevity} \mathrm{of} \mathrm{the} \mathrm{probe.} \mathrm{Based} \mathrm{on}$

\footnotetext{
${ }^{64}$ Venkatasami, G.; Sowa, J.R. A Rapid, Acetonitrile-Free, HPLC Method for Determination of Melamine in Infant Formula. Analytica Chimica Acta 2010, 665, 227-230.

${ }^{65}$ Fiber optic probe specification obtained from: http://us.mt.com/us/en/home.html\# (accessed May 1, 2011 ).

${ }^{66}$ IUPAC, Compendium of Chemical Terminology, 2nd ed. (the "Gold Book") (1997)

${ }^{67}$ MacDougall, D.C.; Warren B. Guidelines for Data Acquisition and Data Quality Evaluation in Environmental Chemistry . Analytical Chemistry 1980, 52, 2242-49.
} 
the research it was determined that the LOD and LOQ levels for the DiComp probe were found to be $1.7 \times 10^{-4} \mathrm{M}$ and $5.7 \times 10^{-4} \mathrm{M}$, respectively. This implies that the LOD level is $0.02 \mathrm{mg} / \mathrm{ml}$. Refer to Figure $4-1$. Thus, the optics of the ATR-IR system will need to be enhanced in future research in order to achieve a lower limit of detection level. The goal is to increase the sensitivity so that the system is able to detect one $\mathrm{mg}$ of an active drug in a 500 $\mathrm{ml}$ vessel volume or $0.002 \mathrm{mg} / \mathrm{ml}$. The ratio of placebo to active will play an important role in the sensitivity and will need to be considered during the analysis. 


\section{Figure 4-1 Limit of detection of ATR-IR system}

Salicylic LOD_Linearity for Salicylic Acid (Concentration vs. Absorbance Band Intensity): Instrument $L O D=0.02 \mathrm{mg} / \mathrm{ml} ;$ Article claim: $0.03 \mathrm{mg} / \mathrm{ml}$

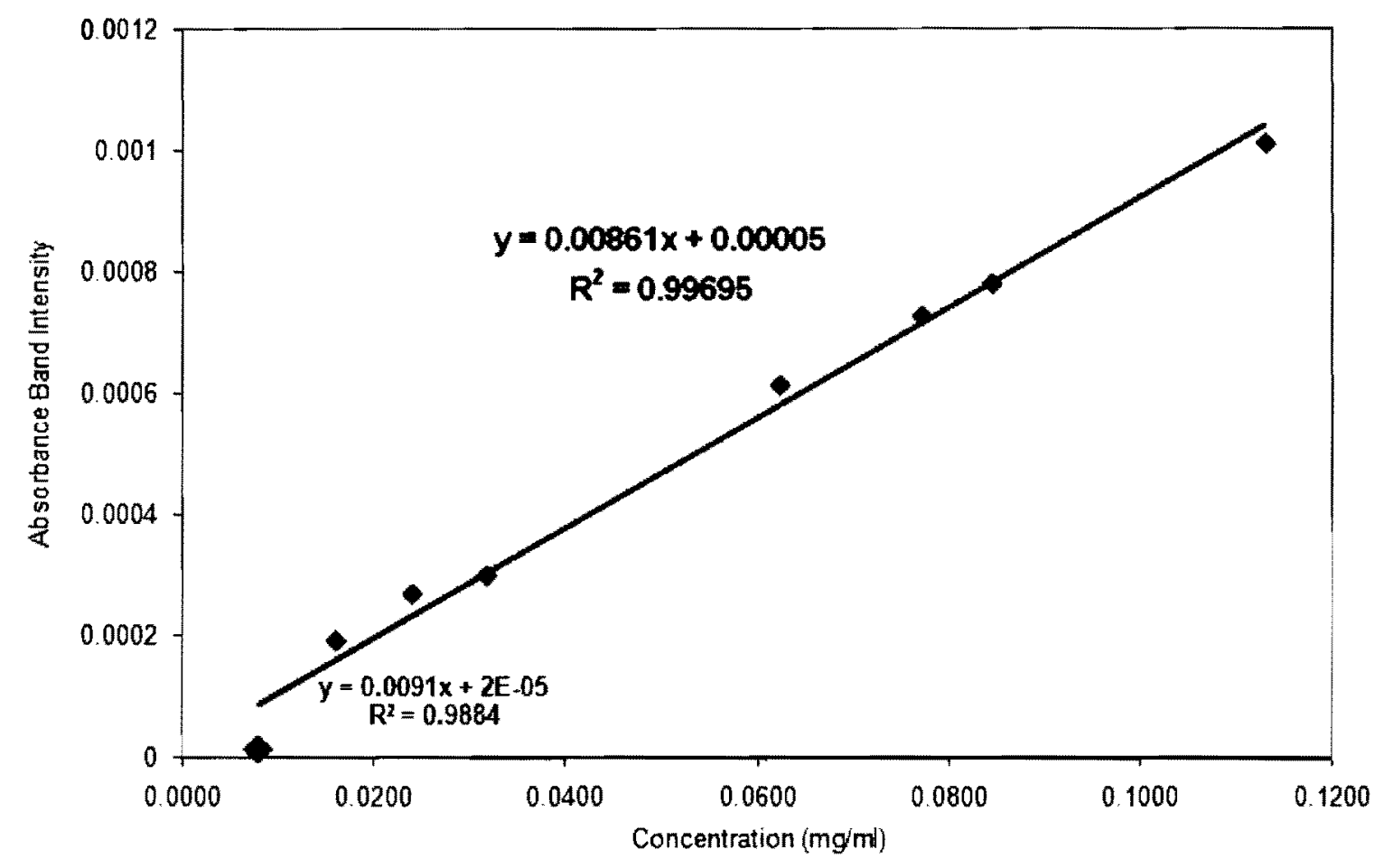


Figure 4-2 Fiber optic probes specifications (ref 65)

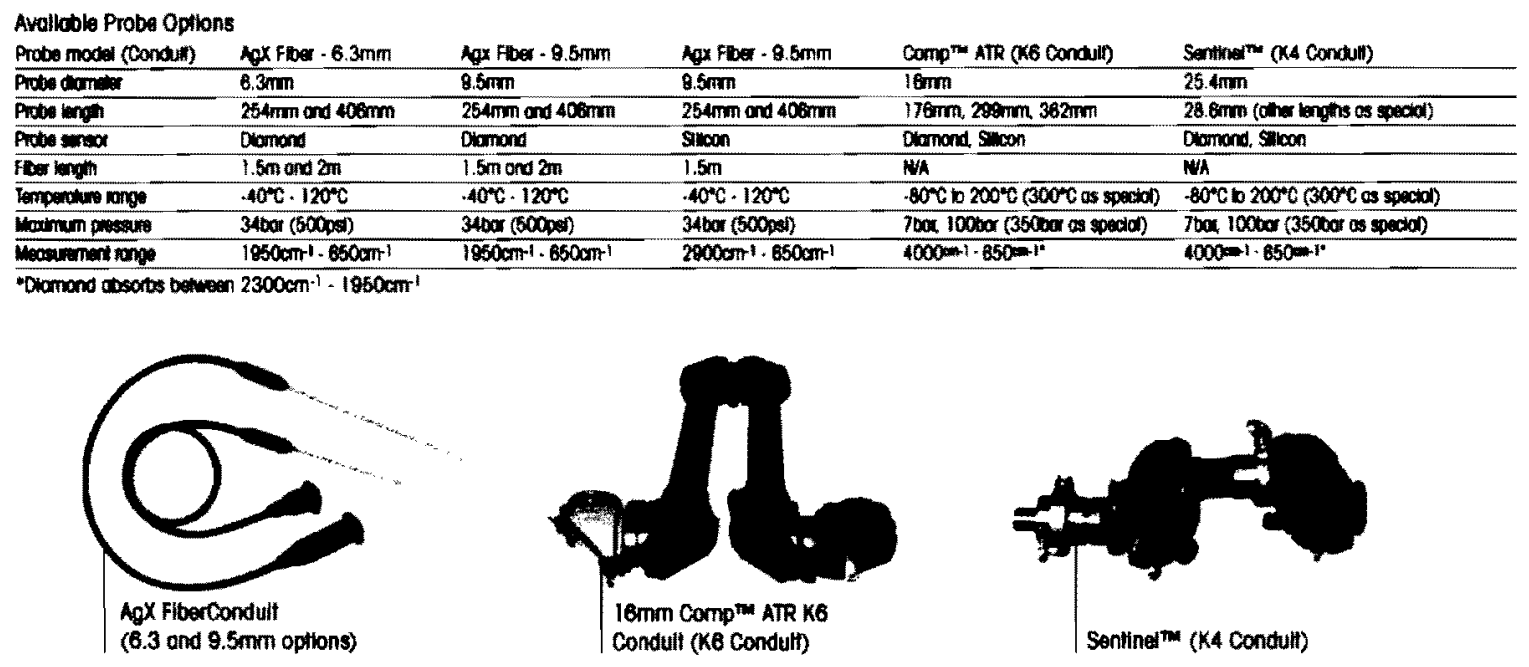


Figure 4-3 Calculated LOD and LOQ values

\begin{tabular}{|c|c|c|c|}
\hline Description & \multicolumn{3}{|c|}{ LOD and LOQ Results for iC10 IR System Probes } \\
\hline IR Probe & K4 Probe & Di Comp Probe & Flow Cell \\
\hline S/N & 2 & 13 & 23 \\
\hline LOD (mM) & $0.63 \mathrm{mM}$ & $0.17 \mathrm{mM}$ & $0.10 \mathrm{mM}$ \\
\hline LOQ (mM) & $2.11 \mathrm{mM}$ & $0.57 \mathrm{mM}$ & $0.33 \mathrm{mM}$ \\
\hline & & & \\
\hline & & & \\
\hline
\end{tabular}


Figure 4-4 LOD/LOQ Sample Calculation Sheet- DiComp Probe Results-1

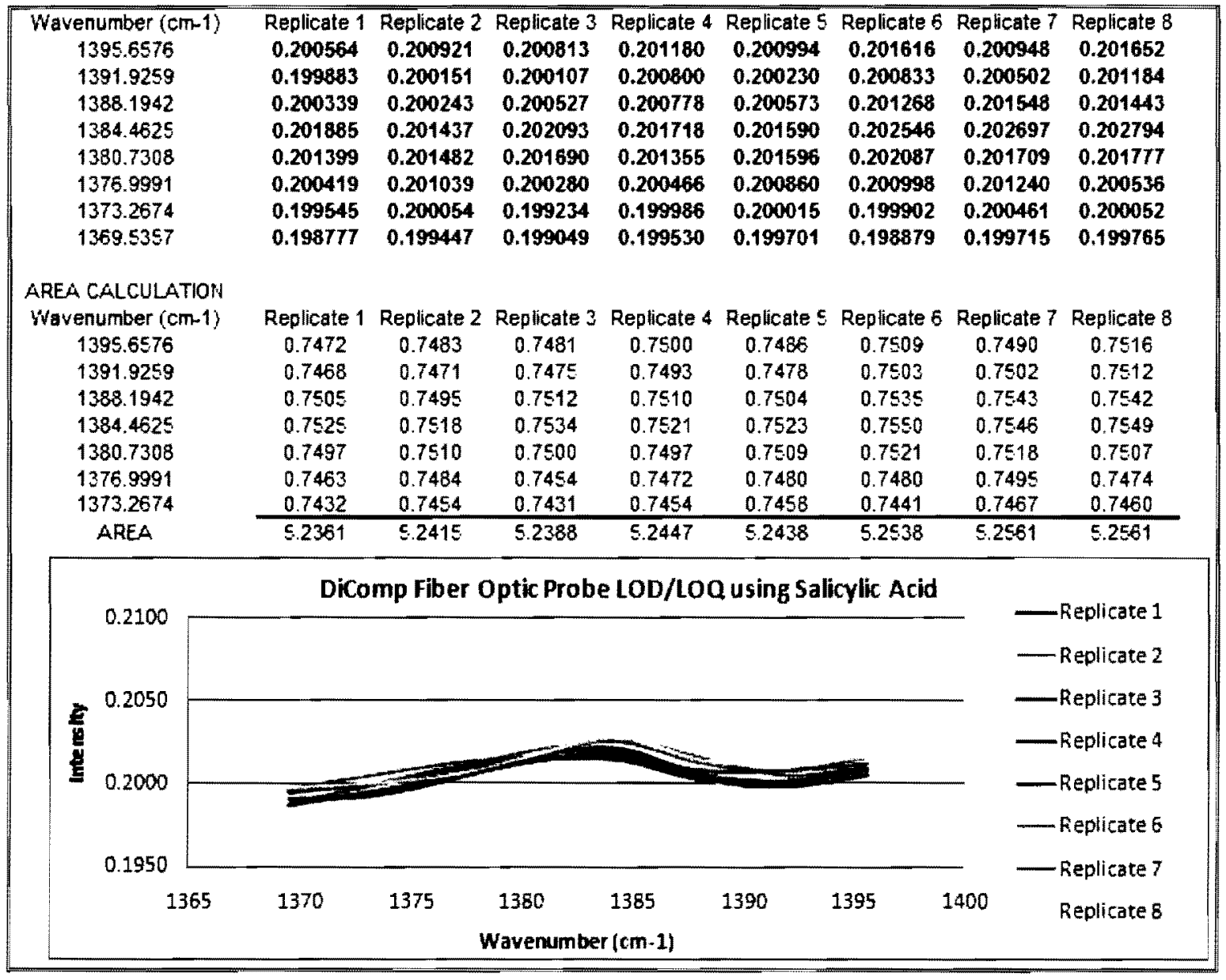


Table 4-1 LOD/LOQ Sample Calculation Sheet- DiComp Probe Result-2

Title: LOD/LOQ Calculations

\begin{tabular}{|c|c|c|}
\hline Row Number & Column A & Column B \\
\hline 1 & Analyte & Salicylic Acid \\
\hline 2 & Method & LOD LOO \\
\hline 3 & Date & Nov. 27. 2010 \\
\hline 4 & Instrument & iC10 Di Comp Probe \\
\hline 5 & Spike & 0.01594 \\
\hline 6 & Units & $\mathrm{mg} / \mathrm{mL}$ \\
\hline 7 & Replicate 1 & 5.23610 \\
\hline 8 & Replicate 2 & 5.2415 \\
\hline 9 & Replicate 3 & 5.2388 \\
\hline 10 & Replicate 4 & 5.2447 \\
\hline 11 & Replicate 5 & 5.2438 \\
\hline 12 & Replicate 6 & 5.2538 \\
\hline 13 & Replicate 7 & 5.2561 \\
\hline 14 & Replicate 8 & 5.2561 \\
\hline 15 & Mean & 5.2464 \\
\hline 16 & Std. Dev & 0.0079 \\
\hline 17 & LOD & $0.02 \mathrm{mg} / \mathrm{mL}$ \\
\hline 18 & LOQ & $0.08 \mathrm{mg} / \mathrm{mL}$ \\
\hline
\end{tabular}


Figure 4-5 LOD/LOQ Sample Calculation Sheet- Flow Cell Probe Results-1

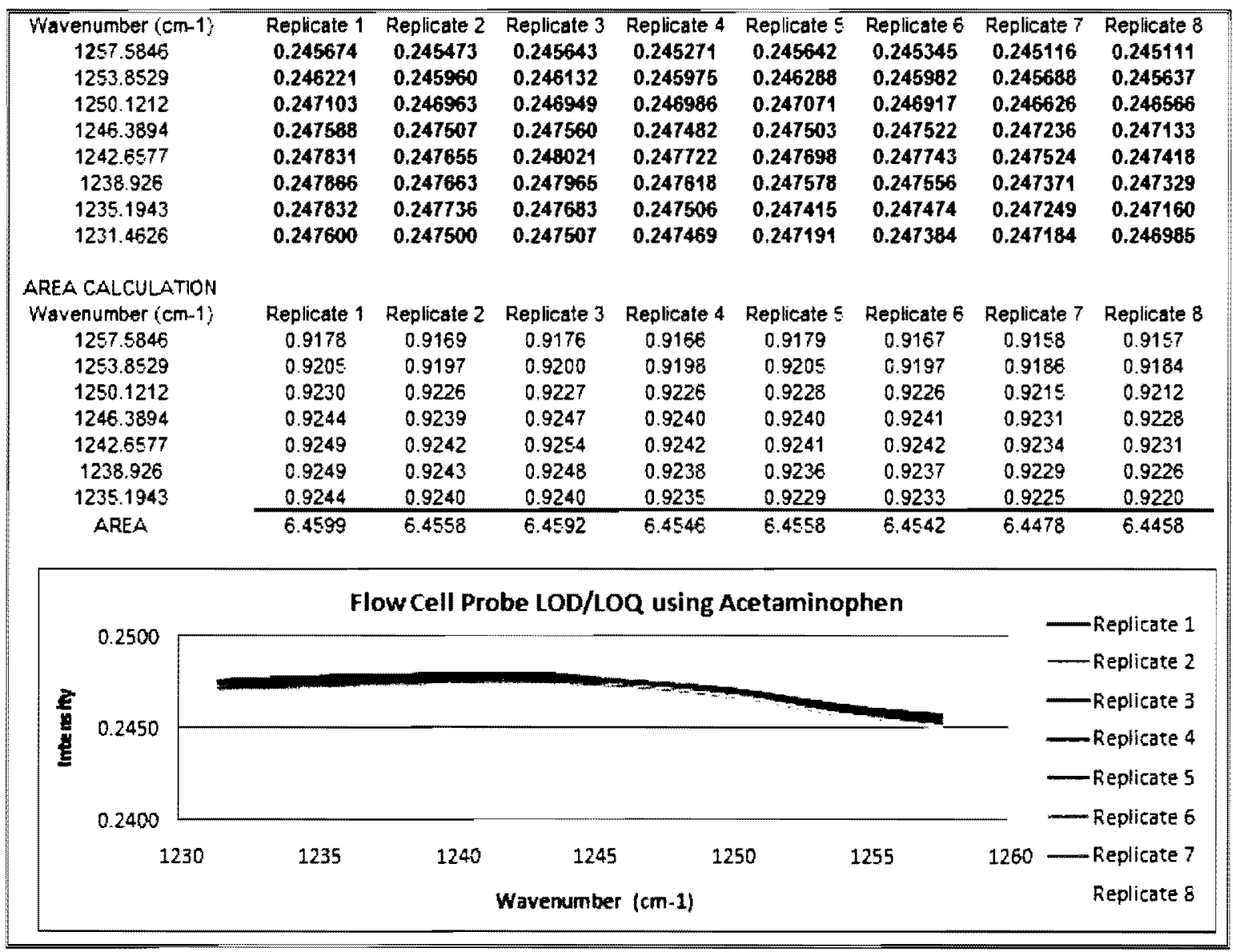




\subsection{Instrument design}

The proposed instrument is illustrated in Figure 4-7. It has ten overall features which are grouped into the following units: I) Optical Path which includes the source, fiber optic cables, ATR crystal, and detector, II) Six Solvation Vessels including the overhead stirrers, reaction vessels and temperature control, and, III) Instrumentation Control and data acquisition/processing software. The choice of six solvation vessels comes from a statistical requirement that is frequently found encountered in complicated experiments where agreement of $n=6$ experiments is considered to provide a statistically robust analysis. ${ }^{68}$ Although chemists are frequently satisfied with one reproducible yield or agreement of three measurements of a rate constant, applied applications of chemistry frequently require more rigorous statistics.

Some regulatory agencies require statistical agreement of six simultaneous solvation studies. Thus, to make the instrument more statistically rigorous and competitive with related instruments on the market, it is important that we use $n=6$ reaction vessels. Also note that a circular arrangement of the solvation vessels is proposed as this design is takes up less space relative to a linear or block arrangement.

We will assemble our new instrument from commercially available components. Thus, our main role will be to select the most appropriate components, to make sure that they properly communicate and have reliable computer software for instrument control data acquisition and processing. Our target specifications are:

\footnotetext{
${ }^{68}$ a) Wilson, E. B. An Introduction to Scientific Research; Dover Press: New Jersey, 1991. b) Dumont, M. L.; Berry, M. R.; Nickerson, B. Probability of passing dissolution acceptance criteria for an immediate release tablet. Journal of Pharmaceutical and Biomedical Analysis 2007, 44(1), 79-84.
} 
1) Sensitivity (as defined as limit of detection, where signal: noise ratio $=3: 1$ ) $<10^{-6} \mathrm{M}$.

2) Sample time $<1$ min per solvation vessel.

3) Wavelength range: $4000-500 \mathrm{~cm}^{-1}$.

4) Temperature control $<+/-0.5^{\circ} \mathrm{C}$ and range -20 to $100^{\circ} \mathrm{C}$.

5) Chemical stability: aqueous solvent conditions ( $\mathrm{pH} 1-12$ ), common organic solvents and tested for safe use of volatile organic solvents such as diethyl ether when used in a fume hood.

6) Although this is a scientific proposal, we desire that the instrument will be marketable, thus, we will aim for a retail cost of $\$ 200 \mathrm{~K}$. 
Figure 4-6

Diagram of proposed Solv-IR Instrument

A diagram of the proposed instrument is shown below.

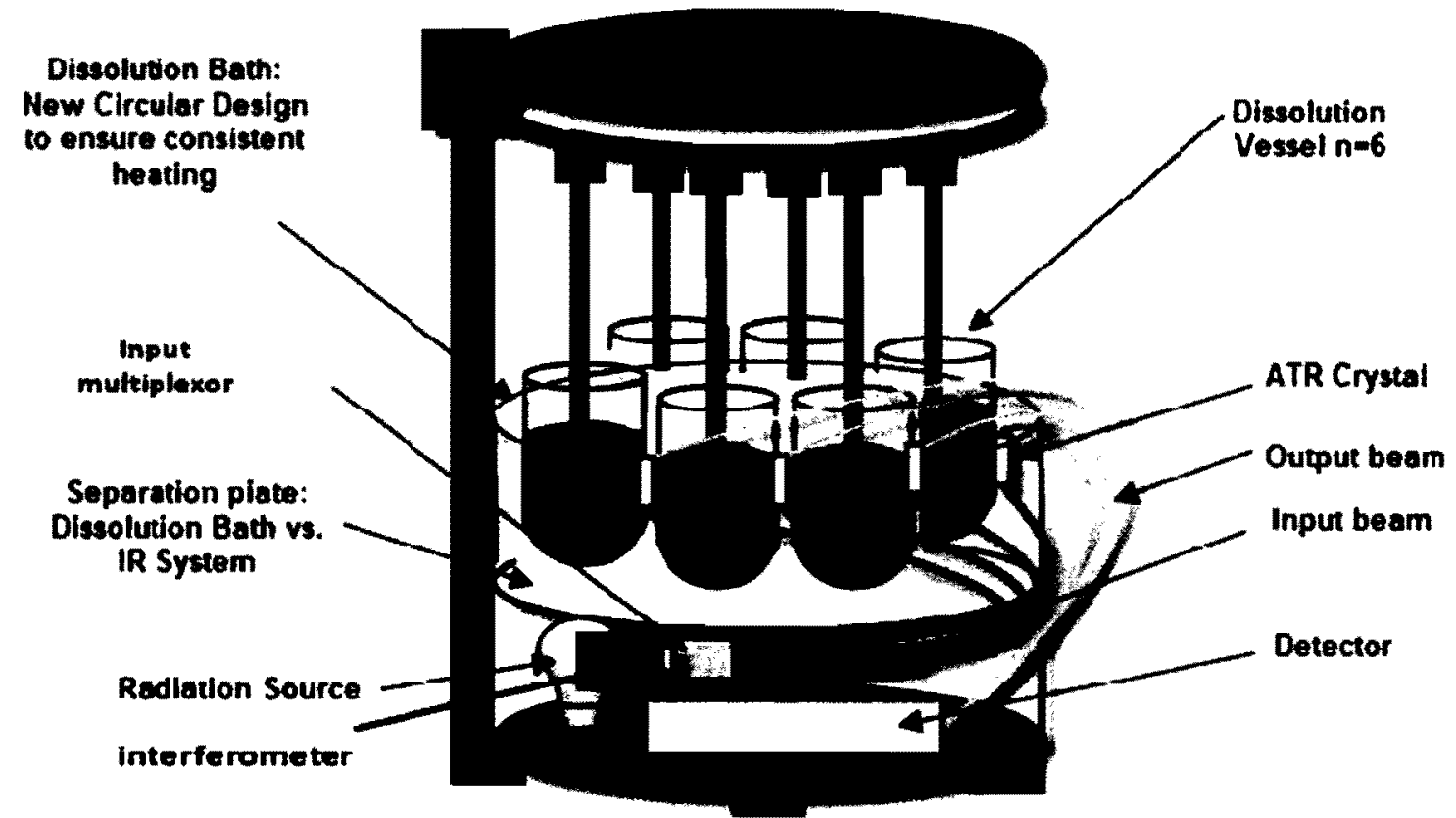




\subsection{System components}

\subsubsection{Radiation source}

This is an encased source for IR radiation consisting of an IR element and Zn-Se lens. A suitable unit is available from Oriel, Apex Infrared Source, Model 66471 (7" H x 12" W x 10" D).

\subsubsection{Interferometer}

This is necessary to modify the source IR light to a broad range of frequencies. Interferometers provide for rapid acquisition and signal averaging. Potential vendors include BrukerOptics. However, an interesting alternative for initial investigations will be to salvage components from a surplus IR (we have two units in disrepair in our department with interferometers).

\subsubsection{Two Six-device Multiplexors}

These devices channel the light from the source so that the maximum amount of light passes through each vessel and then directs the absorbed light to the detector. They are sequential devices such that input/output light spends a defined amount of time at each vessel.

\subsubsection{Six ATR Probes}

Each probe consists of an input fiber optic cable, an ATR crystal and an output fiber optic cable. The cables are chalcogenide fibers that perform well in the mid-IR range and are sufficiently flexible so they can be arranged within the instrument and connected to the ATR 
crystal. Potential suppliers are also RoMack Corp. and Hellma Scientific. The ATR crystal is a $\mathrm{Zn}-\mathrm{Se}$ crystal will provide the desired spectral window $\left(4000-500 \mathrm{~cm}^{-1}\right)$; however, since $\mathrm{Zn}-\mathrm{Se}$ is sensitive to acids and easily abrased, we will identify a suitable coating. So far, the best choice is a diamond coated $\mathrm{Zn}$-Se crystal which provides excellent chemical stability. The narrower spectral range $1900-600 \mathrm{~cm}^{-1}$ is a satisfactory compromise because the "fingerprint" region frequently provides an excellent array of absorption bands. Pike Technologies provides an excellent selection of ATR and coated ATR crystals.

\subsubsection{Detector}

The goal is to obtain a single detector sensitive to $10^{-6} \mathrm{M}$. Currently, the most sensitive detectors are liquid nitrogen-cooled $\mathrm{HgCdTe}$ (MCT) detectors. However, if technology advances, we hope to incorporate a sensitive ambient temperature detector such as Peltierstabilized DTGS (deuterated triglycine sulfate) detector. Commercial vendors include BrukerOptics.

\subsubsection{Six Solvation Vessels}

Preliminary work can be performed on glass $1 \mathrm{~L}$ vessels that we have in stock. We will need to hire a glass blower (ChemGlass, Vineland, $\mathrm{NJ}$ ) to modify each vessel with a rectangular fitting capable of holding rhombohedral ATR crystal as shown in Figure 4-7. 
Figure 4-7 Sketch of ATR crystal embedded in the side wall of the $1 \mathrm{~L}$ glass vessel. The area directly under the stir shaft is known to have poor mixing and as indicated by the term "dead zone".

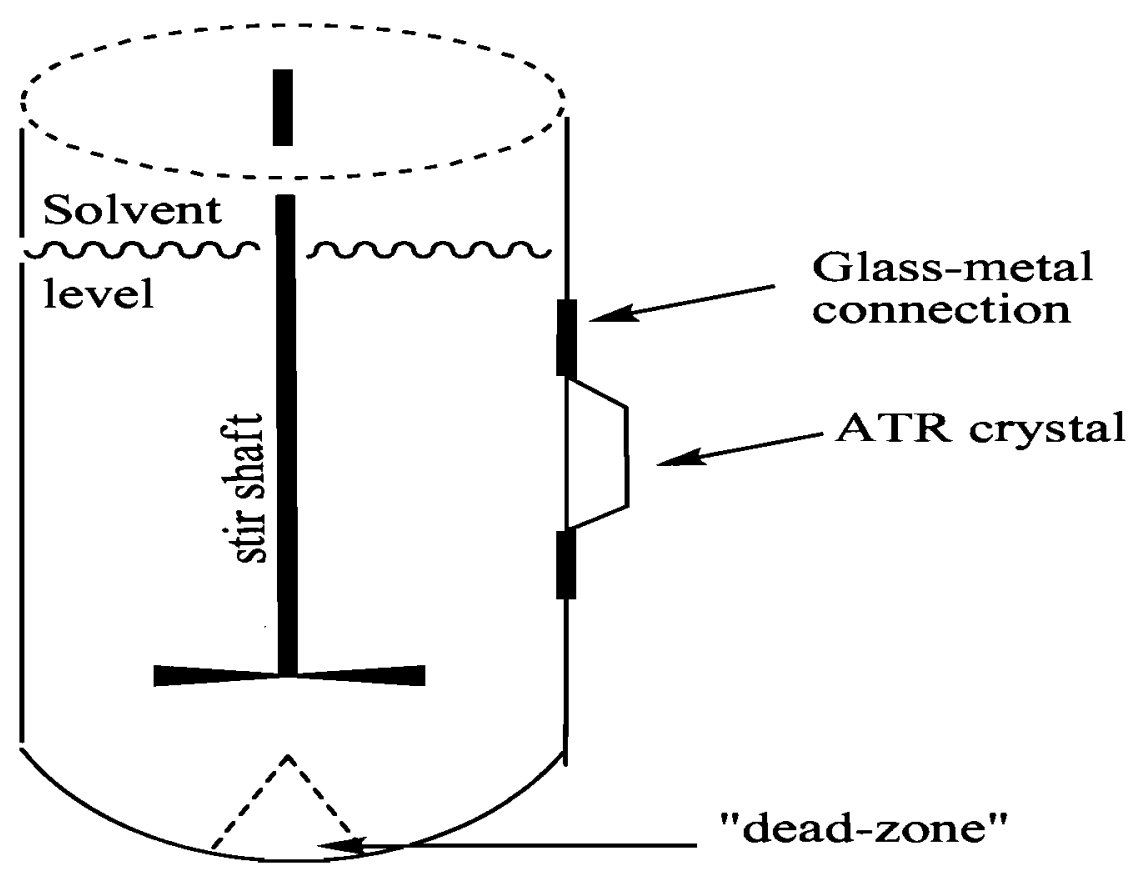


Alternatively, polyethylene and fluoropolymer vessels are available which will be easier to modify especially in prototypes. Quality Lab Accessories (QLA) provides an excellent catalog of glass and polymer vessels, caps and stir shafts. Controlled overhead stirring is performed by a simple gear assembly (Figure 4-8).

Temperature control will be provided by assembling a water bath around the solvation vessels. However and ideal alternative is provide a thermally-conductive jacket around each vessel with which temperature is controlled (for example, see Distek's Evolution 6100 Bathless apparatus. ${ }^{69}$

\footnotetext{
${ }^{69}$ Evolution 6100 - Distek Bathless Dissolution Testing Equipment, Distek Home Page [Online], http:/ www.distekinc.com/products/dissolution evolution 6100.htm (accessed on January 26, 2011). 
Figure 4-8 Top plate of constant rpm stirring assembly showing motor, main gear and minor gears which drive the stir shafts in each vessel

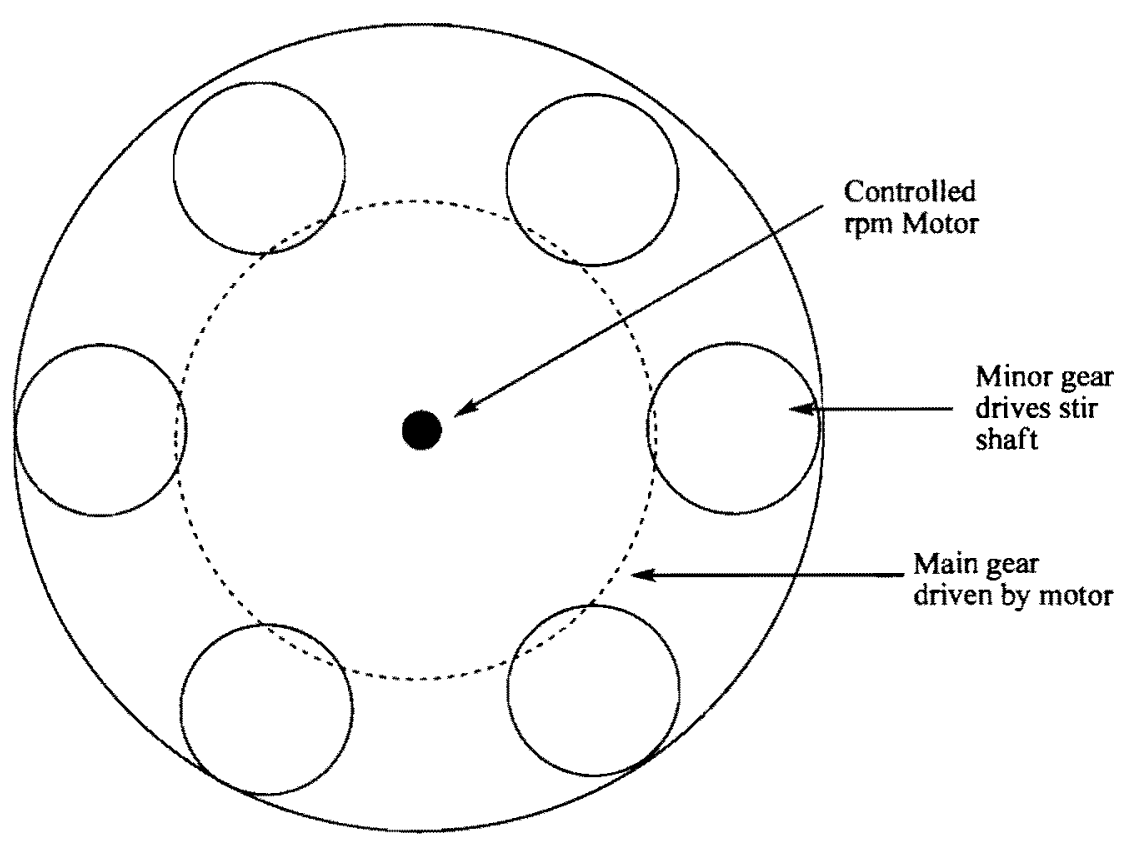




\subsection{Instrument Control/ Acquisition/ Processing.}

Software packages to perform these operations have been well-designed by a number of vendors we will seek an opportunity to license their software and hire a consultant to modify the software package.

\subsection{Anticipated technical difficulties}

i. It may be difficult to match items from different vendors. Solution: try to use one or two primary vendors. For example, BrukerOptics has MATRIX and TENSOR IR spectrophotometers which allow multiple ATR probe attachments. If either of these meet sensitivity requirements, then it would be best to build the Solv-IR from one of these units. A quote for the MATRIX-MF instrument including spectrophotometer, ATR-probes and software has been obtained to support this approach.

ii. It may be difficult to achieve sensitivity within a reasonable time frame if all six vessels are to be measured with one spectrophotometer. Solution: assemble an apparatus with two or three spectrophotometers.

iii. It may be difficult to achieve sensitivity with a vessels are to be measured with one spectrophotometer. Solution: assemble an apparatus with two or three spectrophotometers.

\subsection{Chapter summary}

The preliminary studies in Chapters 2 and 3 indicate great potential for use of the Solv-IR instrument in pharmaceutical chemistry, especially to study dissolution of pharmaceutical formulations. Chapter 5 will address a potential application of this instrument in the study of the chemistry behind oil spills. 
This instrument development proposal aims to construct a new type of instrument that will allow the study of solvation of solutes in aqueous and organic systems. The main feature of the instrument design is the use of infrared (IR) spectroscopy to study this dynamic behavior. Traditionally, IR is not considered to be a quantitative technique. However, using ATR crystals ensures that the technique is most certainly quantitative. Also, IR is traditionally not considered to be a sensitive method, however, our preliminary results indicate detection limits of $10^{-4} \mathrm{M}$. Employing better optics and detection will improve sensitivity to our target value of $10^{-6} \mathrm{M}$. The instrument has an additional feature which may seem strange to most chemists as it will have six reaction vessels. This feature is important for statistical reasons and for marketing reasons. Solvation is a chemically and mechanically complex process. To ensure accurate studies of the solvation process, it is important to have a large array of data such that statistically significant interpretations can be made. In addition, competing instruments that use UV-Vis technology have six reaction vessels so they conform to certain regulatory requirements.

We propose to assemble the instrument using commercially available components. We will build to spectroscopic part of the instrument starting with this unit. In addition, we feel that we work with their data acquisition software to incorporate instrument control and data processing. Finally, we will construct the reaction vessels, stirring function and thermal control and integrate this unit with the spectroscopy unit to have a fully functioning integrated Solv-IR instrument.

Students working on this project will obtain valuable experience on working with electrical, optical and mechanical parts and tools which is quite rare for a chemistry student. However, 
Seton Hall University

since this is all focused on discovering new ways to study the fundamental and practical aspects of solvation chemistry, this will be an exciting and worthwhile challenge. While assembling the instrument students will be able to investigate chemical phenomenon in a way that has been previously inaccessible. 


\section{$5 \quad$ Analysis of the BP Gulf oil spill by Attenuated Total Reflection-Infrared Spectroscopy and Dissolution}

This chapter focuses on describing the scientific research that was conducted on the recent BP oil spill in the Gulf of Mexico. Moreover, this research was accomplished using the React-IR/ dissolution system. Although the BP oil spill occurred outside of New Orleans, Seton Hall University employed its faculty members, students and research facilities toward understanding this environmental disaster.

A general background on the BP oil spill will be provided to gain more understanding of the subject matter, which will be useful when discussing the results later in the chapter. The BP oil spill (also referred to as the Deepwater Horizon oil spill, Gulf of Mexico oil spill or Macondo blowout), is the largest accidental marine oil spill in the history of the industry. The Deepwater Horizon was a nine-year drilling unit that was floating in the Gulf of Mexico. Refer to Figure 5-1 for a photo of Deepwater Horizon before the accident occurred. The spill occurred on April 20, 2010 due to an explosion of the Deepwater Horizon. This led to a loss of 4.9 million barrels of crude oil to the ocean. As a result, the spill caused extensive damage to the fishing and tourism industries around the Gulf of Mexico. 
Seton Hall University

Figure 5-1 Photo of oil rig similar to Deepwater Horizon ${ }^{70}$

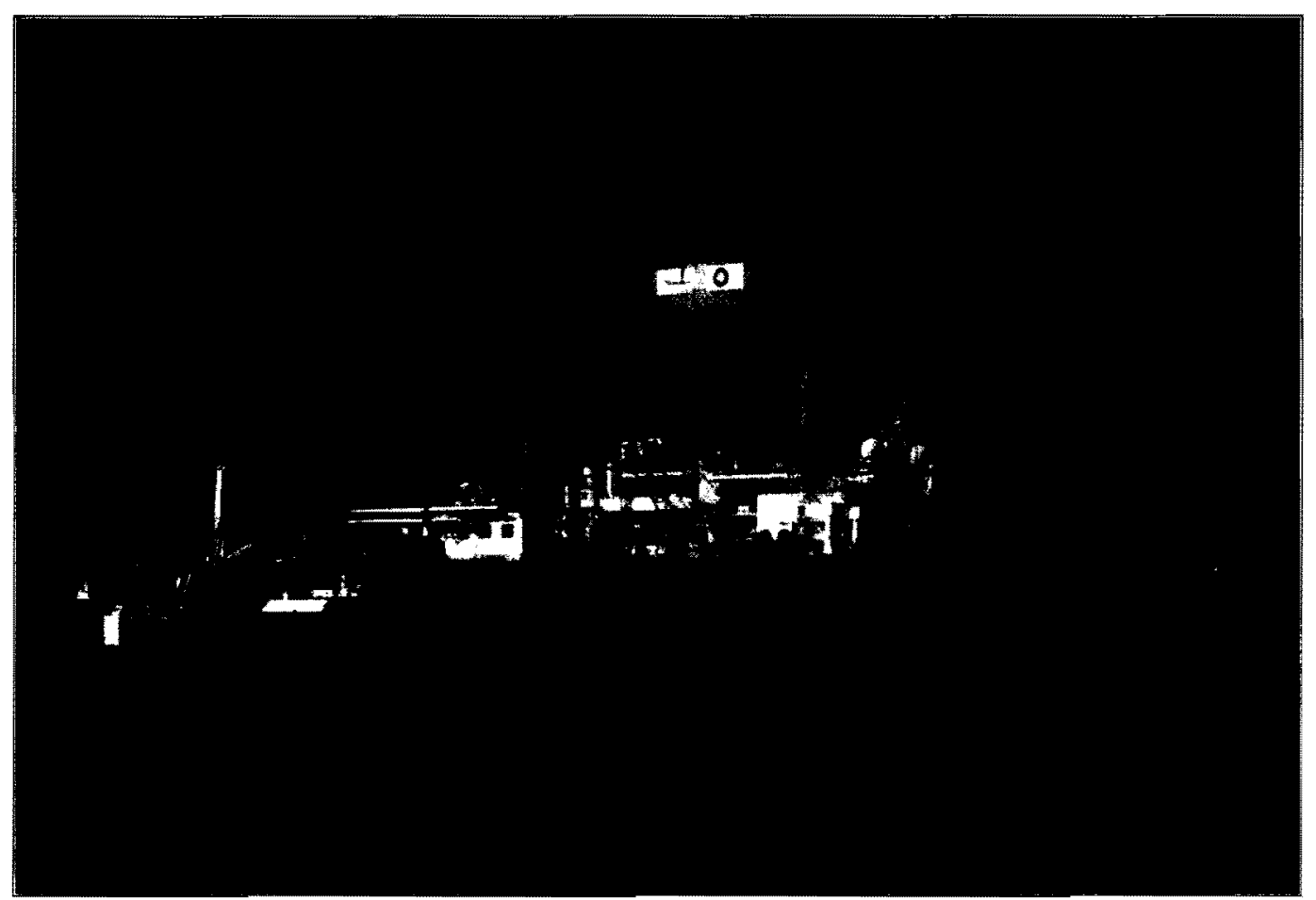

${ }^{70}$ Photo obtained from Lauren Michael Ridley during her NOAA trip to Gulf (obtained July 2011).

[113] 


\section{PETROLEUM AND CRUDE OIL}

Both crude oil and natural gas are mostly made up of hydrocarbons. The lighter hydrocarbons which include methane, ethane, propane and butane occur as gases, while the heavier hydrocarbons (i.e., pentane and higher) occur in the form of liquids or solids. The hydrocarbons in crude oil are mostly alkanes, cycloalkanes and several aromatic hydrocarbons. Moreover, other organic compounds contain nitrogen, oxygen and sulfur, and trace amounts of metals such as iron, nickel, copper and vanadium.

\subsection{Research with React-IR}

As part of the multidisciplinary project to understand the effect of the oil spill, the React-IR system coupled to a dissolution system was used to study the dispersion effect of dispersants on crude oil. Dispersants were used to break up the Deepwater Horizon oil spill before it reached the shore. The dispersants are designed to break down the crude oil into tiny drops, which can be mechanically dispersed or consumed by bacteria. The aim is to lessen the impact of giant plumes of crude oil washing onto oyster beds and birds' nests and on the sandy beaches. The dispersants or chemicals used in the BP oil spill could also do more damage than good in the long run. The dispersants are toxic in nature and could have devastating effects on future generations of marine life. The financial cost of using these dispersants could run into the millions. Some of the chemicals used in commercial dispersants ${ }^{71}$ are shown in Table 5-1. This information was obtained from the EPA website.

\footnotetext{
${ }^{7}$ Environmental Protection Agency Home Page. http://www.epa.gov/bpspill/dispersants-qanda.html\#list (accessed May 1, 2011).
} 
Specifically, the system was used to study dispersion of oil in the presence of dishwashing soaps as preliminary models for commercial dispersants. From these preliminary experiments, the research was able to measure the effect of dispersants on petroleum oil. In addition, these preliminary experiments were able to determine the feasibility of the technique in order to move forward with the project at a larger scale. This would include purchasing commercial dispersants. Furthermore, Dawn $\mathbb{R}$ dishwashing soap was used because of its powerful claim to clean wildlife affected by oil spills. Dawn® specifically uses anionic surfactants, such as alkyl dimethyl amine oxide, to break down oil samples. These surfactants reduce the surface tension of water and can weaken the barrier that automatically forms between oil and water, allowing them to unnaturally mix. 
Seton Hall University

Table 5-1 Chemicals used in commercial dispersants (COREXIT 9500 and 9527)

\begin{tabular}{|l|l|}
\hline CAS Registry Number & Chemical Name \\
\hline $57-55-6$ & 1,2 -Propanediol \\
\hline $111-76-2$ & Ethanol, 2-butoxy-* \\
\hline $577-11-7$ & Butanedioic acid, 2-sulfo-, 1,4-bis(2-ethylhexyl) ester, sodium salt (1:1) \\
\hline $1338-43-8$ & Sorbitan, mono-(9Z)-9-octadecenoate \\
\hline $9005-65-6$ & Sorbitan, mono-(9Z)-9-octadecenoate, poly(oxy-1,2-ethanediyl) derivs. \\
\hline $9005-70-3$ & Sorbitan, tri-(9Z)-9-octadecenoate, poly(oxy-1,2-ethanediyl) derivs \\
\hline $29911-28-2$ & 2-Propanol, 1-(2-butoxy-1-methylethoxy)- \\
\hline $64742-47-8$ & Distillates (petroleum), hydrotreated light \\
\hline
\end{tabular}

*This ingredient is present in 9527 but is eliminated in 9500 . 


\subsection{Experimental section}

\subsubsection{Chemical and materials}

Crude oil samples were collected by Dr. John Sowa and University of New Orleans scientists from a location near the city of Venice, Louisiana. Refer to the figures below showing the locations where the oil samples were collected. The University of New Orleans faculty collected samples from the Gulf of Mexico. The location of the samples collected was determined by GPS at the following location: $28^{\circ} 53.412^{\prime} \mathrm{N}, 89^{\circ} 08.780^{\prime} \mathrm{W}$. Methanol, acetone and acetonitrile (HPLC grades) were purchased from Pharmaco-Aaper. Dawn $\mathbb{B}$ Antibacterial and Ultra Joy (lemon scent) dishwashing detergent soaps were used as the dispersants for this research. All solutions were prepared using water treated by a Milli-Q Millipore purification system. All purified water aliquots have resistivity of not less than 18 MOhm- $\mathrm{cm}^{-1}$. 
Figure 5-2 Aerial view: ${ }^{72}$ location of BP Gulf oil samples

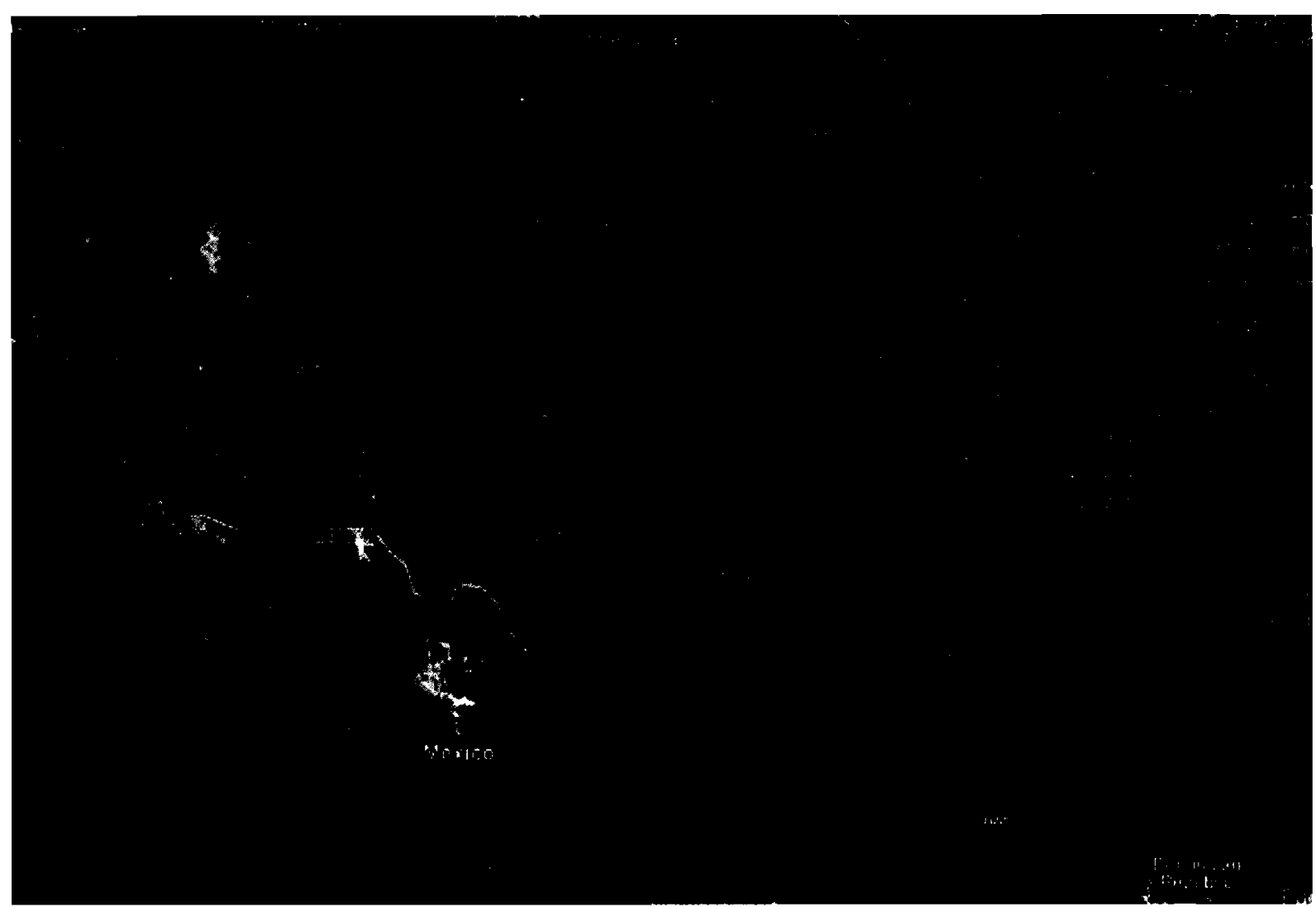

${ }^{72}$ Google Maps: http://maps.google.com/maps?hl=en\&tab=wl (accessed May 1, 2011). [118] 
Seton Hall University

Figure 5-3 Zoom view: location of BP Gulf oil samples [Both samples were collected near the UNO site]

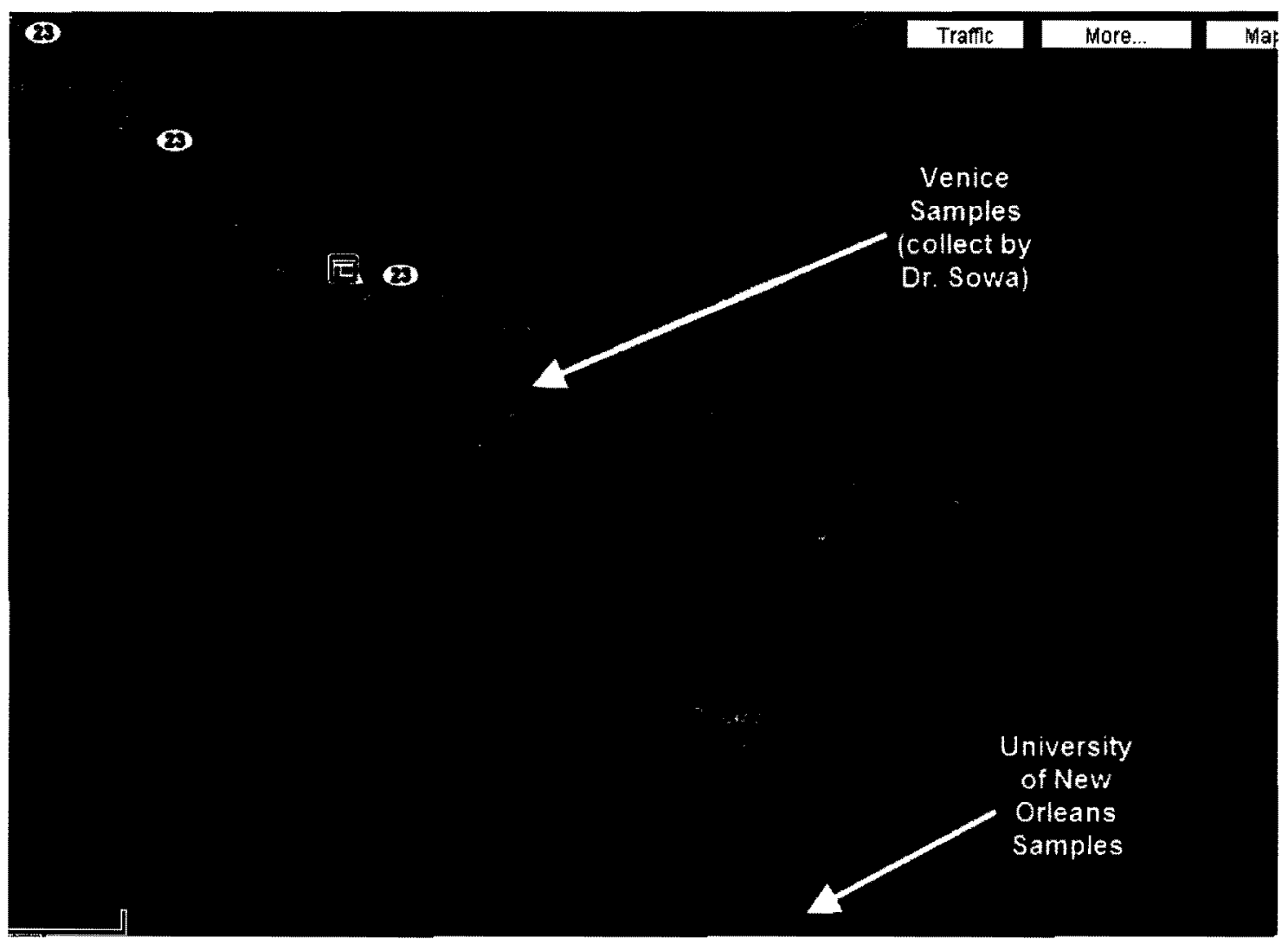


Figure 5-4 Ocean depth diagram ${ }^{73}$ showing location of BP Gulf oil samples (reproduced and modified from Ref 73)

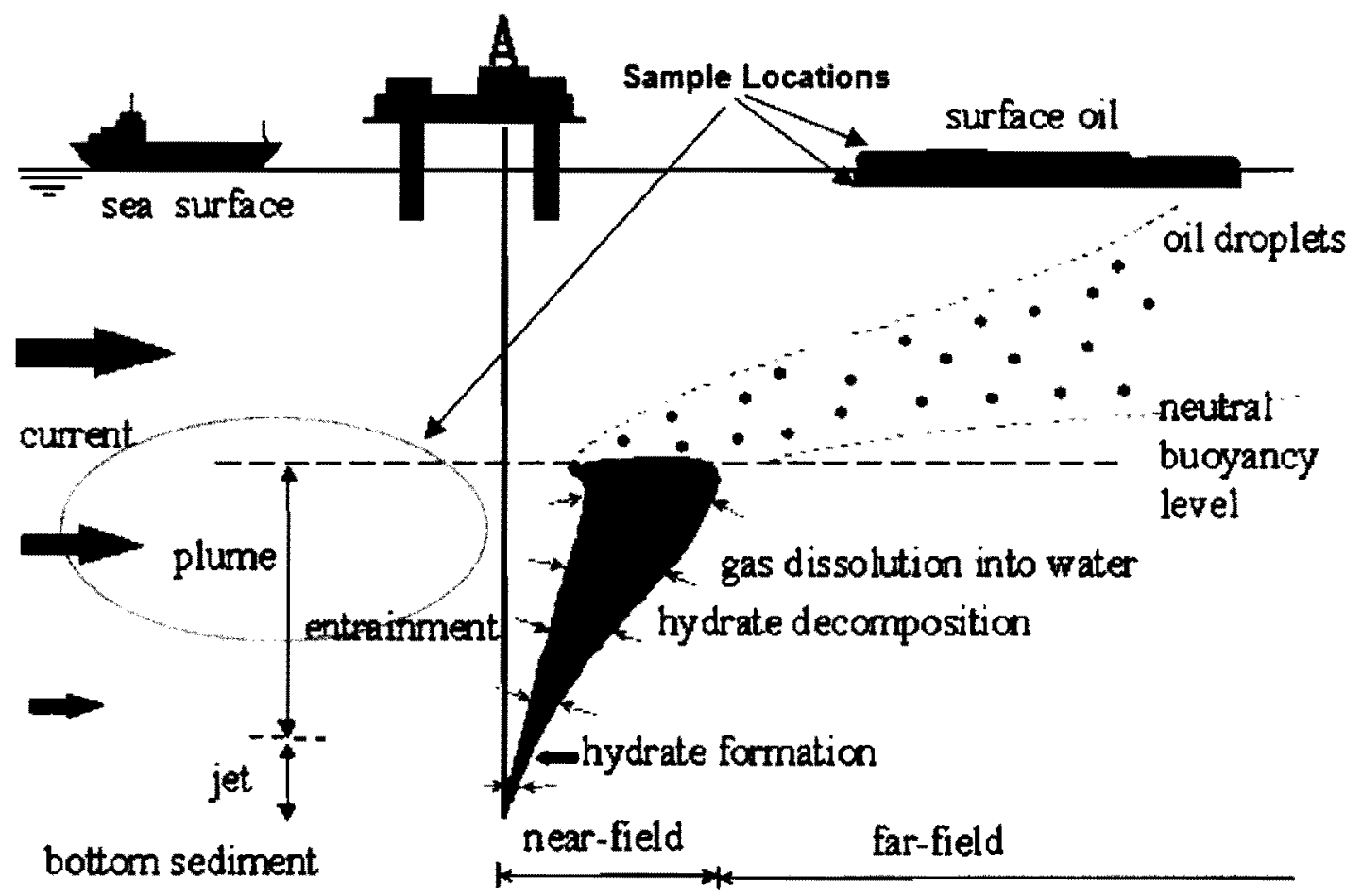

\footnotetext{
${ }^{73}$ Figure obtained from http:/www.crrc.unh.edu/final/Yapa2003final/figurel.jpg (accessed May, 2011).
} 


\subsubsection{Instrumentation}

Samples were tested using Mettler Toledo's iCl0 FT-IR system using a fiber optic probe equipped with a $1 \mathrm{~mm}$ diamond coated ATR probe. The IR system was operated by Mettler Toledo's iC IR version 3.0 or 4.0 software. All dissolution experiments were tested using a Vankel Dissolution Bath (model no. 700). All UV/Visible testing was carried out using HP 8453 spectrophotometer. All analytical weight measurements were carried out using Mettler Toledo's DeltaRange and AG204 DeltaRange.

Figure 5-5 Instrument photo: ATR-IR and dissolution apparatus

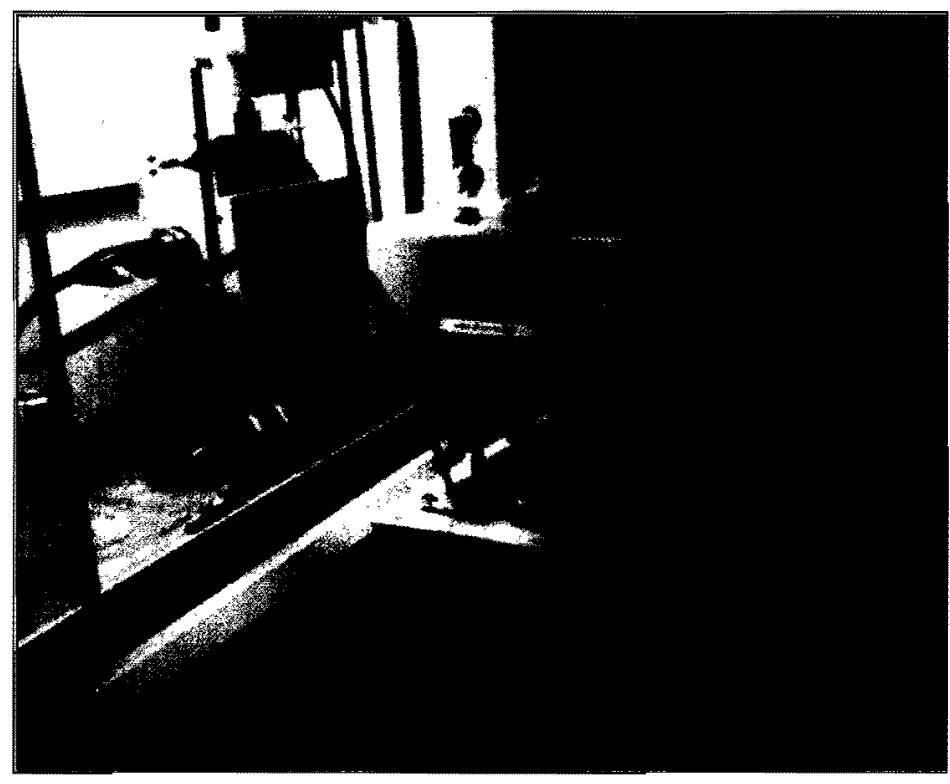


Seton Hall University

Table 5-2 Experimental details and sample information

\begin{tabular}{|l|l|l|}
\hline No. & Parameter & Setting \\
\hline 1 & Vessel volume & $900 \mathrm{~mL}$ \\
\hline 2 & Medium & Distilled water \\
\hline 3 & Agitation speed & $100 \mathrm{rpm}$ \\
\hline 4 & Temperature & $25^{\circ} \mathrm{C}$ \\
\hline 5 & Dawn®/ Joy® Dishwashing detergents & $0.2 \%$ solutions \\
\hline 6 & Dissolution bath & VanKel VK700 \\
\hline 7 & ATR-IR & Mettler Toledo iC 10 \\
\hline
\end{tabular}




\subsubsection{Dispersant stock solution}

A $0.2 \%$ solution of Dawn $($ dishwashing solution was made up $(0.2 \mathrm{~mL}$ was added to approximately $120 \mathrm{~mL}$ of distilled water). A $0.2 \%$ solution of Joy dishwashing solution was made up ( $2 \mathrm{~mL}$ into $1000 \mathrm{~mL}$ of distilled water).

\subsubsection{Dawn® ingredients:}

Water, Sodium Lauryl Sulfate, Sodium Pareth-23, Sulfate C-12-14-16, Dimethyl Amine Oxide, SD Alcohol, Undeceth-9, Propylene Glycol, Cyclohexandiamine, Polyacetate, Protease, Fragrance, FD\&C blue, no phosphate.

\subsubsection{Joy ingredients:}

Water, Sodium Lauryl Sulfate, Sodium Pareth-23, C12-14-16 Dimethyl Amine Oxide, SD Alcohol 40-B, Undeceth-9, PPG-26, Sodium Chloride, Cyclohexanediamine, Polyacetate, Fragrance, FD\&C Yellow \#5, D\&C Red No. 33

\subsubsection{Crude oil sample preparation}

The dispersion experiments were carried out by adding different amounts of Dawnß dishwashing detergent in vessels containing $900 \mathrm{~mL}$ of distilled water and five (5) grams of semi-solid oil sample. Photographs of the oil dispersion studies were collected at different time points throughout the runs. The dispersion experiments were carried out over two hours. 


\subsubsection{ATR-IR analysis}

The ReactIR ${ }^{\mathrm{TM}}$ iC10 FTIR instrument is composed of an MCT detector (liquid nitrogen cooled) and the FiberConduit ${ }^{\mathrm{TM}}$. The FiberConduit ${ }^{\mathrm{TM}}$ is comprised of flexible IR transparent silver chloride/silver bromide optical fibers. The fiber optic probe interface (AgX $9.5 \mathrm{~mm} \mathrm{x}$ $1.5 \mathrm{~m}$ Fiber (Silver Halide)) contains a diamond tip-DiComp ATR crystal. The resolution was set to 8 wavenumbers. The optical range used by the system is: $1900 \mathrm{~cm}^{-1}$ to $650 \mathrm{~cm}^{-1}$. The gain adjustment was set to normal $(1 \mathrm{x})$ and the apodization method was set to Happ-Genzel. The system uses compressed air (house air, filtered and de-humified) to purge the optics.

\section{$5.3 \quad$ Results}

The experiments in this chapter demonstrate how ATR-IR may be used in the study of treatments of oil spills. During the BP oil spill, the use of dispersants at the well-head and on the ocean surface was extremely controversial. Thus, this research was interested in finding ways to evaluate this treatment process. Although the commercial dispersants were not used in this research, dishwashing soaps were used as preliminary models. The results show that it takes considerable time for the oil to be broken down by the dishwashing soaps. Controlled dissolution experiments were carried out in an attempt to simulate the dispersion of oil as it occurred during the oil spill. The dissolution experiments were tested over the course of several hours and were held under elevated agitation conditions and at $25^{\circ} \mathrm{C}$. These parameters were used in order to simulate the conditions of the ocean water in the Gulf of Mexico. In all of the dispersion experiments a known amount of crude oil was added to the dissolution vessel. Dispersants were then added and the experiments were monitored over several hours. During this time several photos were taken to compare and record the size of 
the crude oil samples. Moreover, the ATR-IR probe was placed in the vessel as a way to monitor the release of oil within the vessel as a consequence of dispersant in solution.

\subsubsection{Standards and expected absorption values}

Standard spectra of crude petroleum oil were obtained during this phase of the research. Crude oil is expected to have prominent IR bands at $1375 \mathrm{~cm}^{-1}$ (due to methyl groups) and $1465 \mathrm{~cm}^{-1}$ (due to methylene groups). Refer to Table 5-3. Also, blank ocean solutions were tested to confirm there was no interference at these two FTIR bands. As shown in Figure 5-6 below, the crude oil samples obtained by Dr. Sowa and the faculty of University of New Orleans show prominent peaks at 1375 and $1465 \mathrm{~cm}^{-1}$. Moreover, ocean samples obtained from regions under the surface oil did not show these two bands. 
Seton Hall University

Table 5-3 Expected FT-IR stretching values for crude oil and sulfonated soaps

\begin{tabular}{|l|l|l|}
\hline $\begin{array}{l}\text { Functional } \\
\text { group }\end{array}$ & Absorption $\left(\mathrm{cm}^{-1}\right)$ & Description \\
\hline $\mathrm{CH}_{2}$ & $1465 \mathrm{~cm}^{-1}$ & Methylene groups have a characteristic bending \\
\hline $\mathrm{CH}_{3}$ & $1375 \mathrm{~cm}^{-1}$ & Methyl groups have a characteristic bending \\
\hline $\mathrm{SO}_{2}$ & $1150-1165 \mathrm{~cm}^{-1}$ & Symmetric stretch \\
\cline { 2 - 3 } & $1342-1352 \mathrm{~cm}^{-1}$ & Asymmetric stretch \\
\hline
\end{tabular}


Figure 5-6 Gulf oil vs. blank (ocean water) standard spectra

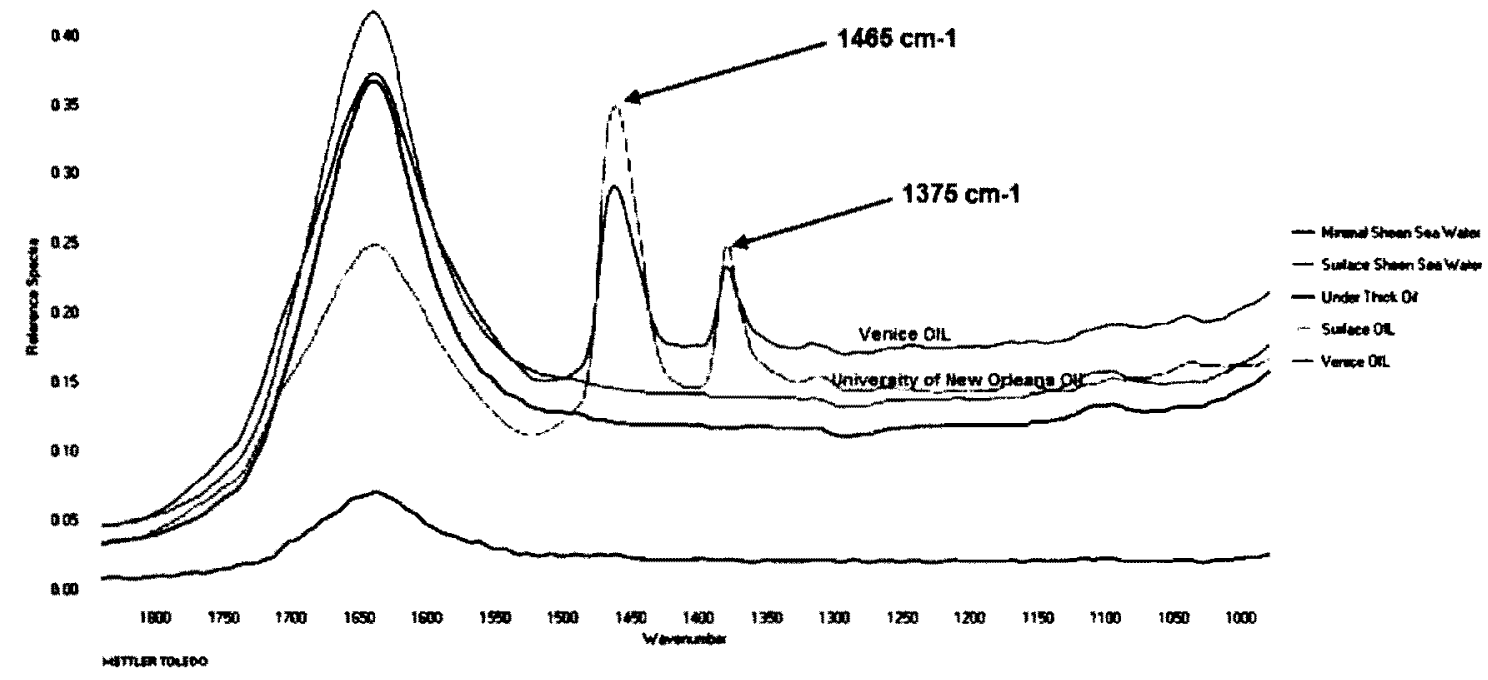


Figure 5-7 Baseline corrected: ATR-IR scans of BP oil and Ocean Water samples

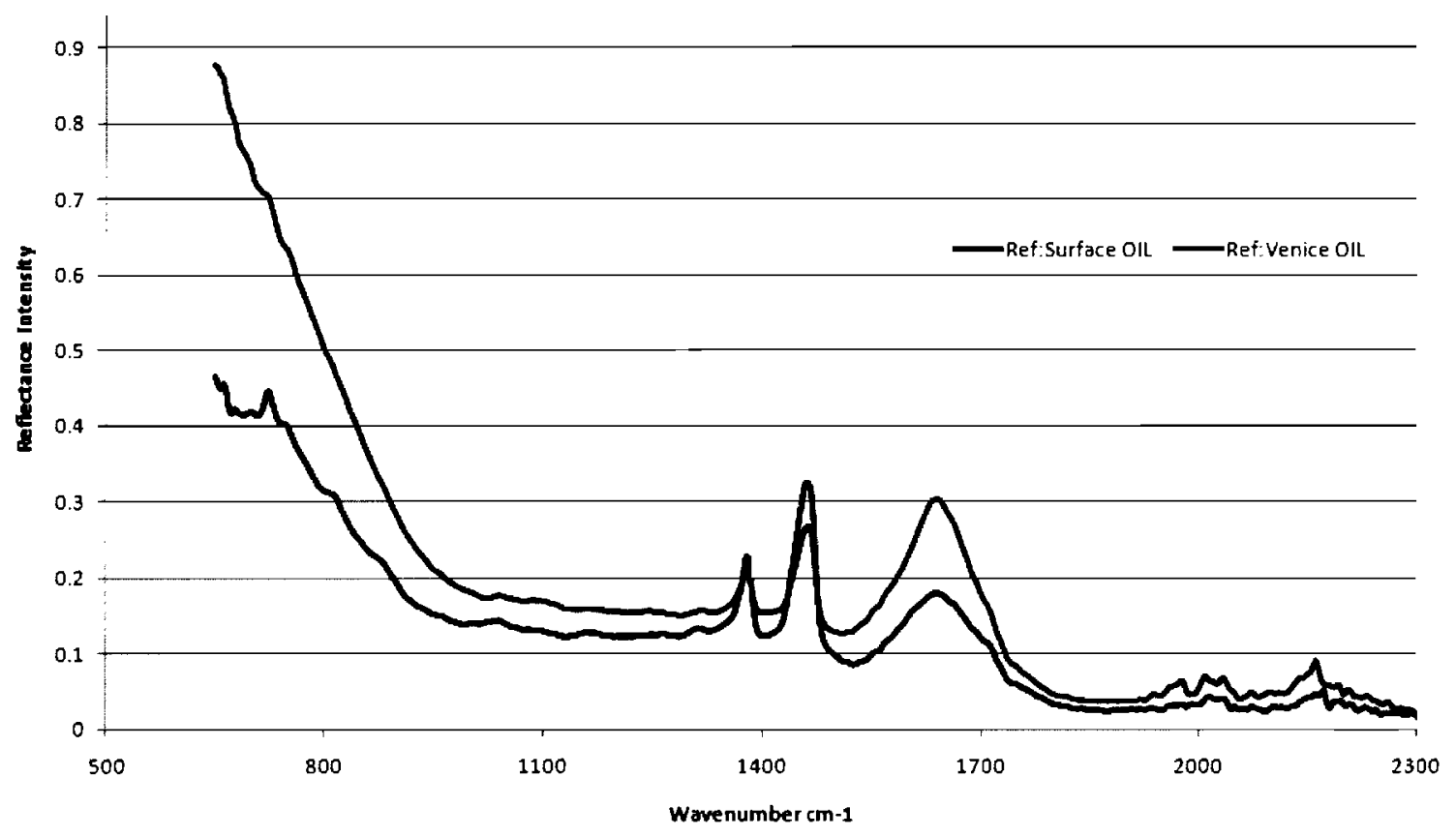


Figure 5-8

Photo of vessels at different time points during the dispersion experiments

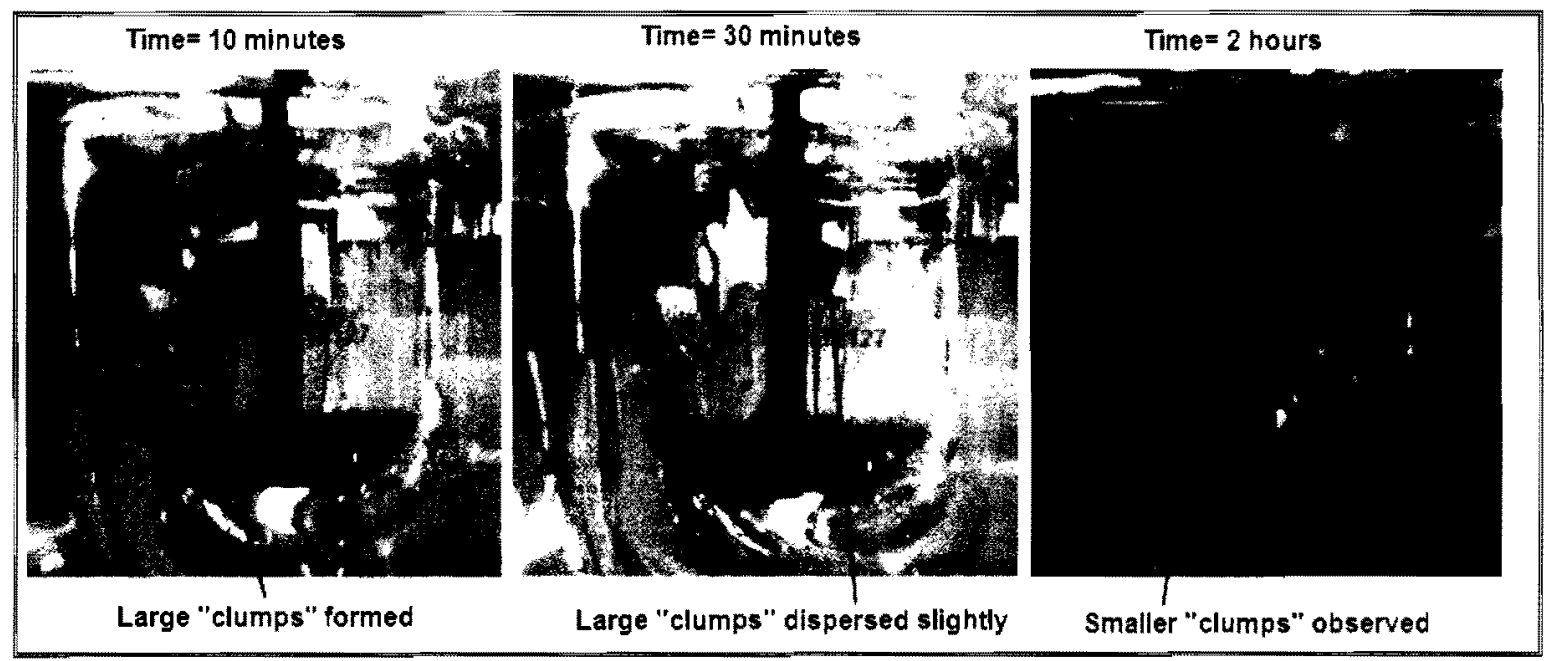




\subsubsection{Venice oil samples dispersed using Joy soap}

During the first phase of experimentation, Joy was used to simulate dispersant. Approximately $50 \mathrm{~mL}$ of the Joy stock dispersive solutions were added to different vessels containing $900 \mathrm{~mL}$ of distilled water and five (5) grams of crude oil collected near Venice, Louisiana. The final dispersant concentrations, compared to the concentration of commercial dispersants used in the ocean, are expected to be higher. The temperature was held constant at $25^{\circ} \mathrm{C}$. The agitation speed was set to $100 \mathrm{rpm}$. Unfortunately, the Joy dispersant solution contained interference peaks at $1465 \mathrm{~cm}^{-1}$ as shown in Figure 5-9 due to the presence of $\mathrm{SO}_{2}$ groups in the soap. 


\section{Figure 5-9 ATR-IR scans of Venice oil and $50 \mathrm{~mL}$ of Joy soap}

This figure displays ATR-IR spectra of a solution containing distilled water, Joy dispersant solution and crude oil collected from Venice, LA. As seen in the figure, there are several peaks observed between $1000 \mathrm{~cm}^{-1}$ and $1500 \mathrm{~cm}^{-1}$. These peaks, moreover, increase with time. As discussed earlier, the peaks at $1375 \mathrm{~cm}^{-1}$ and $1465 \mathrm{~cm}^{-1}$ are indicative of crude oil. These peaks represent the methyl and methylene groups respectively. The other peaks at $1050 \mathrm{~cm}^{-1}$, $1205 \mathrm{~cm}^{-1}$ and $1254 \mathrm{~cm}^{-1}$ were found in the blank solution and were attributed to the phosphates in Joy.

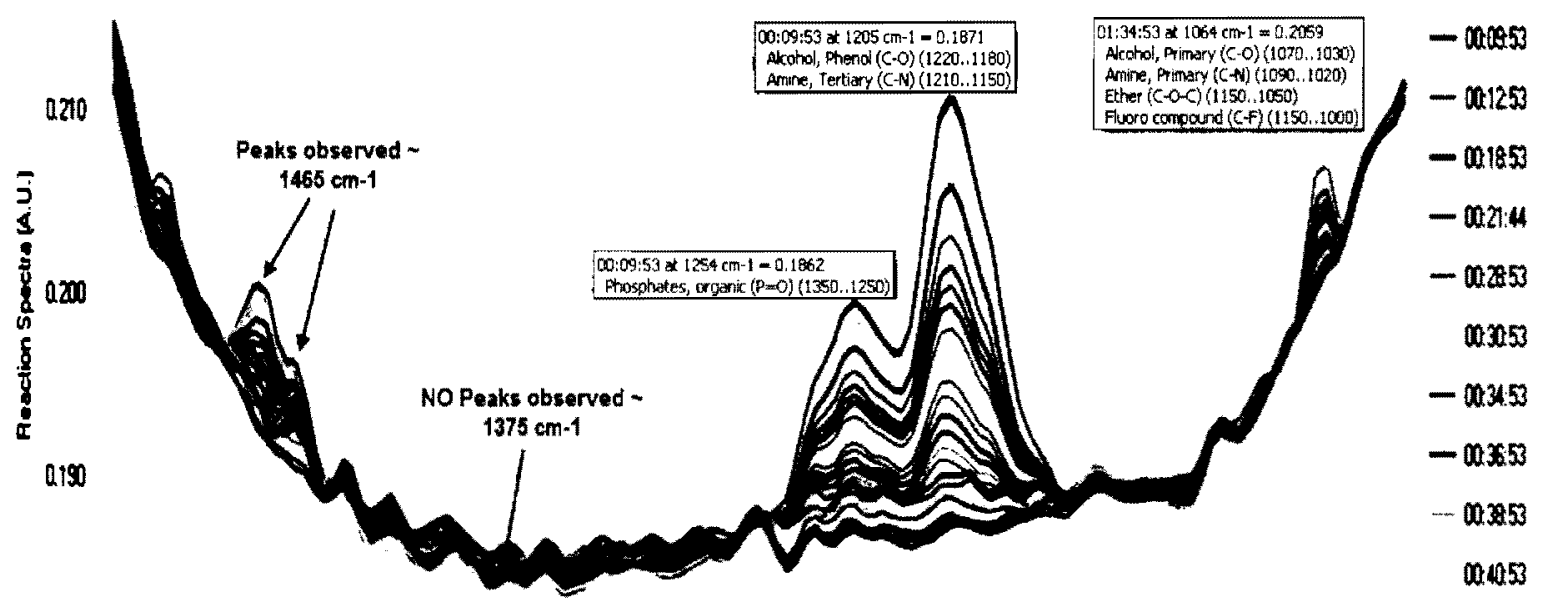

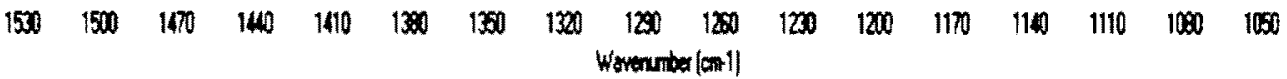


Figure 5-10 ATR-IR scans of $50 \mathrm{~mL}$ of Joy soap only (Blank solution)

This figure displays ATR-IR spectra of a solution containing distilled water and Joy dispersant solution but no crude oil. As seen in the figure, there are several peaks observed between $1000 \mathrm{~cm}^{-1}$ and $1500 \mathrm{~cm}^{-1}$. These peaks, moreover, increase with time. The peaks observed at $1050 \mathrm{~cm}^{-1}, 1205 \mathrm{~cm}^{-1}$ and $1254 \mathrm{~cm}^{-1}$, were attributed to the phosphates in Joy.

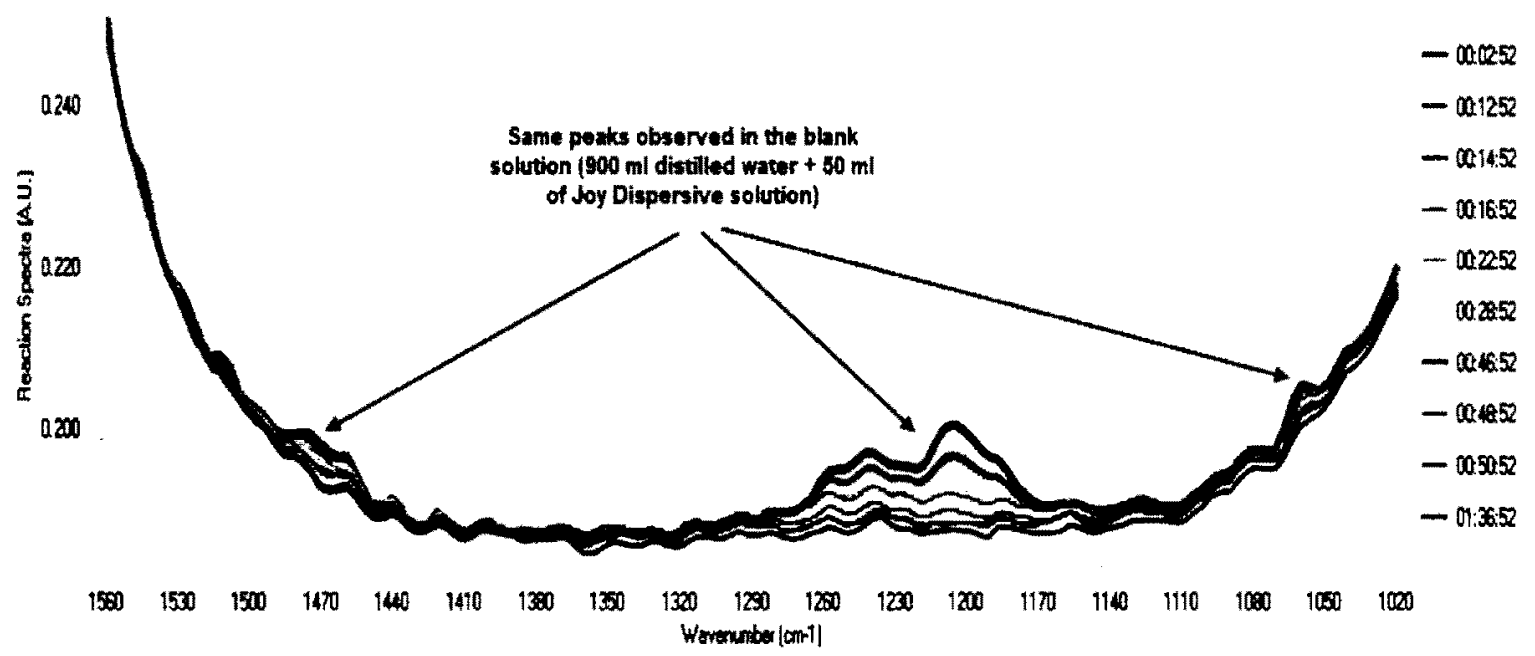




\subsubsection{Venice oil samples dispersed using Dawn® soap}

Dawnß dishwashing solution was used during the second phase of the research. Approximately $50 \mathrm{~mL}$ of stock dispersive solutions were added to vessels containing $900 \mathrm{~mL}$ of distilled water and 5 grams of Venice oil. The final dispersant concentrations, compared to the concentration of commercial dispersants used in the ocean, were expected to be higher. This preliminary model, although not an exact model of the commercial setting, was considered a worst case scenario. It was considered worst case because of the higher concentration of dispersant used in the controlled $900 \mathrm{ml}$ volume setting. The temperature was held constant at $25^{\circ} \mathrm{C}$. The agitation speed was set to $100 \mathrm{rpm}$. Dawn $\otimes$ dispersant solutions did not give interference peaks at $1465 \mathrm{~cm}^{-1}$ or extra sulfonate peaks at $\sim 1200 \mathrm{~cm}^{-1}$. Therefore, Dawn $®$ dishwashing detergent soap was ultimately used to simulate the properties of the dispersant. 
Figure 5-11 ATR-IR scans of Venice oil and $50 \mathrm{~mL}$ of Dawn® soap

This figure displays ATR-IR spectra of a solution containing distilled water, Dawn $(\mathbb{B}$ dispersant solution and crude oil collected from Venice, LA. As seen in the figure, there are several peaks observed between $1140 \mathrm{~cm}^{-1}$ and $1520 \mathrm{~cm}^{-1}$. These peaks, moreover, increase with time. As discussed earlier, the peaks at $1375 \mathrm{~cm}^{-1}$ and $1465 \mathrm{~cm}^{-1}$ are indicative of crude oil. These peaks represent the methyl and methylene groups respectively.

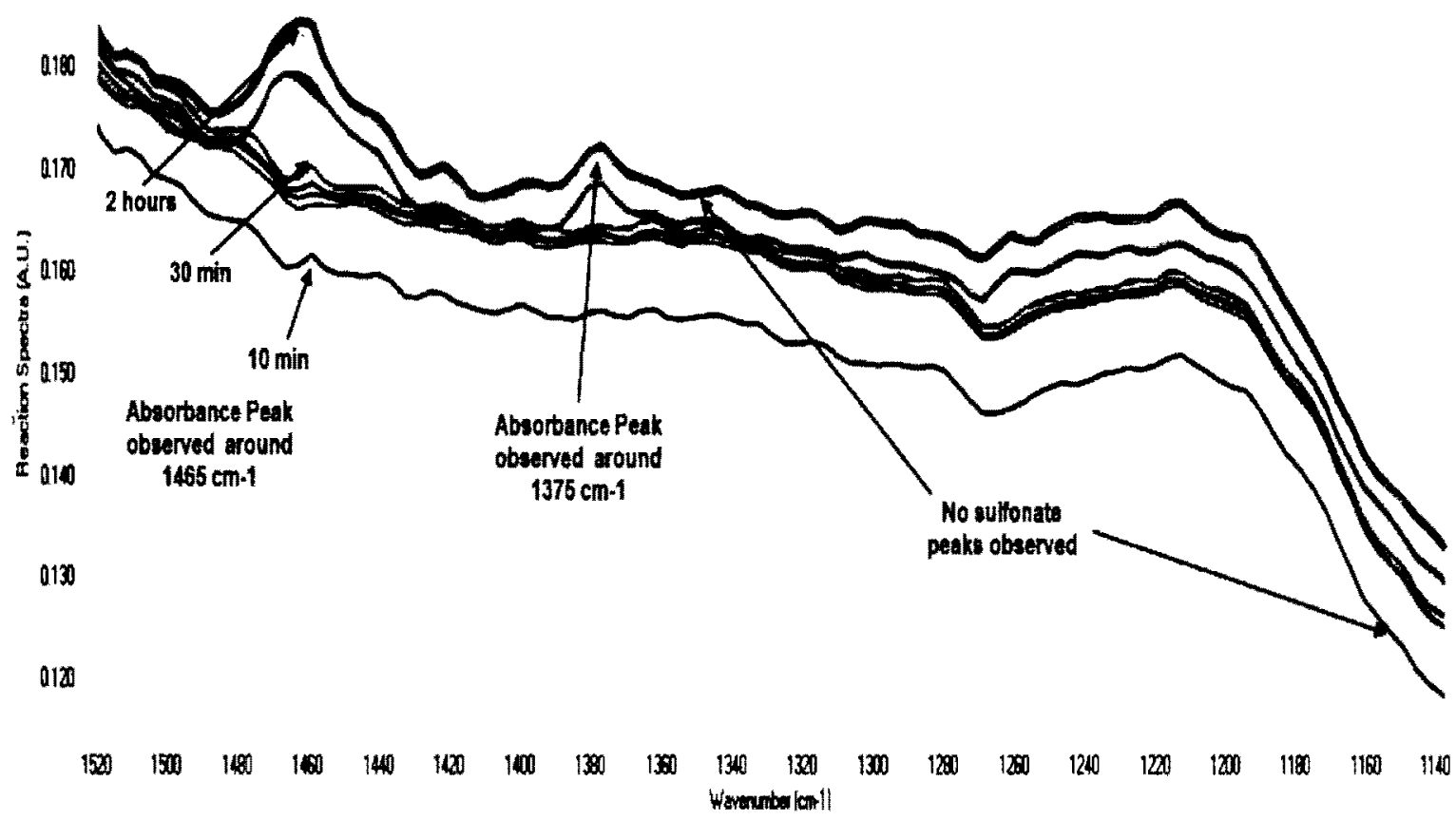


Figure 5-12 ATR-IR scans of $50 \mathrm{~mL}$ of Dawn® soap only (Blank solution)

This figure displays ATR-IR spectra of a solution containing distilled water and Dawn ${ }^{\circledR}$ dispersant solution but no crude oil.

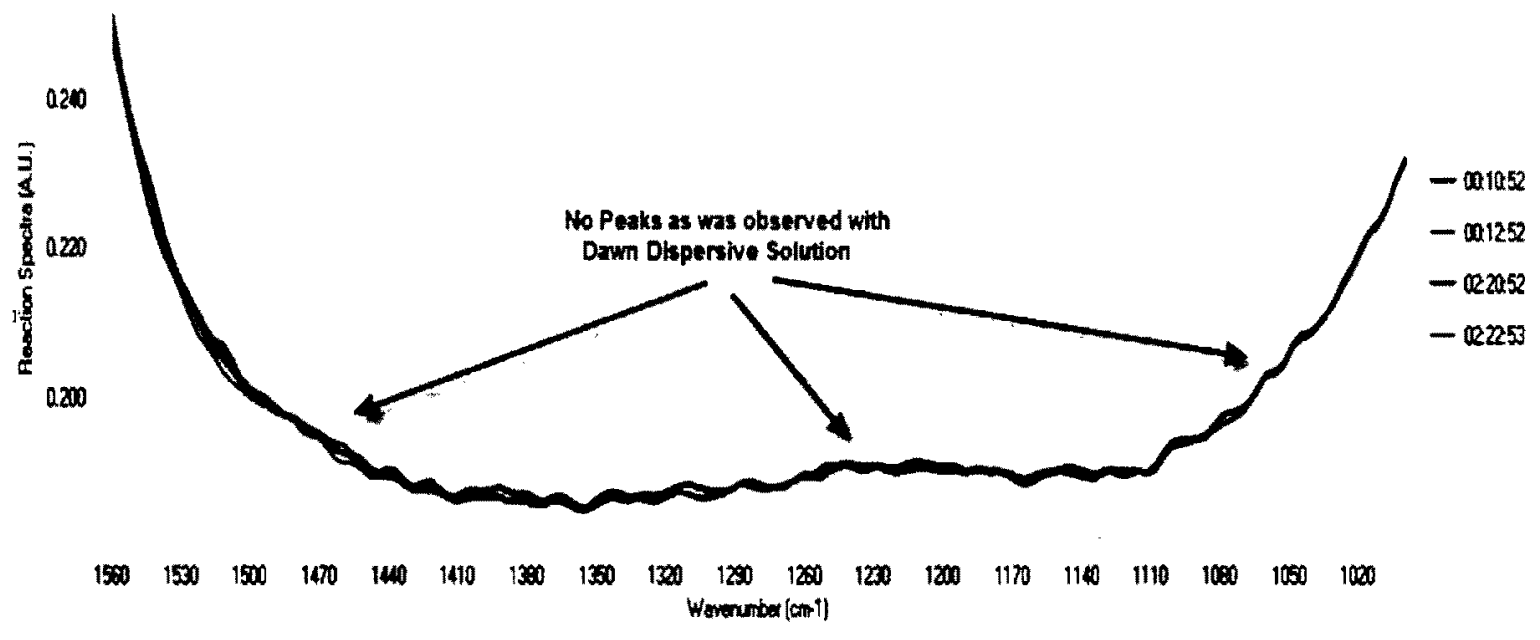




\subsubsection{Percent (\%) dispersant used}

An example of the percent of dispersants used during the research is shown in Table 5-4, Table 5-5 and Table 5-6. First, the ratio of Dawn $®$ sample to the vessel volume is displayed in Table 5-4. This is represented as (A). Next, the ratio of oil to the vessel volume is displayed. This is represented by (B). Last, the ratio of (A) to (B) is shown in Table 5-6, which represents the percent dispersant calculated.

Table 5-4 Amount of Dawn® in solution

\begin{tabular}{|l|l|}
\hline Description & Amount \\
\hline Amount of Dawn® sample in solution* & 0.0017 \\
\hline Total Volume of Dawn® Solution Added (mL) & 54 \\
\hline Total Volume in Vessel (mL) & 954 \\
\hline Ratio of Dawn® sample vs. Volume in Vessel (A) & 0.0001 \\
\hline
\end{tabular}

* Approximately $0.2 \mathrm{ml}$ of Dawn\& Solution was added to approximately $120 \mathrm{ml}$ of distilled water

Table 5-5 Amount of BP oil in solution

\begin{tabular}{|l|l|}
\hline Description & Amount \\
\hline Amount of Oil (Venice Samples) added (grams) & 5 \\
\hline Total Volume in Vessel (mL) & 954 \\
\hline Ratio of Oil Sample vs. Volume in Vessel (B) & 0.0052 \\
\hline
\end{tabular}

Table 5-6 \% Dispersant calculated

\begin{tabular}{|l|l|}
\hline Description & Amount \\
\hline Ratio of Dawn® sample vs. Volume in Vessel (A) & 0.0001 \\
\hline Ratio of Oil Sample vs. Volume in Vessel (B) & 0.0052 \\
\hline \% Dispersant calculated (A / B) & $1.8 \%$ \\
\hline
\end{tabular}


Common dishwashing detergent was found to have little affect on the dispersion of crude oil. As discussed earlier, dissolution experiments configured with IR spectroscopy were carried out whereby aliquots of crude oil were subjected to different brands of dishwashing detergent in order to measure and determine dispersion effect. Moreover, the dissolution experiments were carried out under controlled conditions in an attempt to simulate the Gulf waters. Also, the type of dispersant used played a major role. As shown in the figures earlier, Joy and Dawn® dishwashing detergents were used during this phase of the research. The Joy blanks solutions (did not contain crude oil), however, showed interference peaks at $1170-1270 \mathrm{~cm}^{-1}$ and $1465 \mathrm{~cm}^{-1}$. These bands were associated to the sulfonate functional groups and methylene groups respectively. Dawn® blank solutions, however, did not give interference peaks at

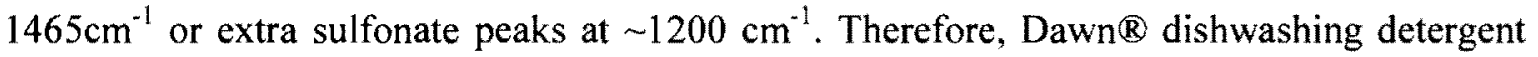
soap was ultimately used to simulate the properties of the dispersant. The dispersion results clearly show that dispersion of crude oil took at least 30 minutes and up to 2 hours.

\subsubsection{Analysis by UV-Vis Spectroscopy}

In addition, the BP Oil samples were tested by UV/Vis spectroscopy. The objective of this part of the research was to determine the amount of porphyrins, if any, in crude oil..$^{74}$ Porphyrins are structurally similar to both chlorophyll and hemoglobin molecules and are destroyed by oxygen and heat. Porphyrins are heterocyclic macromolecules composed of four modified pyrrole subunits interconnected at their alpha carbon via methine bridges. These molecules are aromatic and highly conjugated systems. Porphyrins have very intense 
absorption bands in the visible region. Porphyrins can also contribute to the formation of stable oil-in-water emulsions ${ }^{75}$ which will aid in the dispersion process. Chlorophyll, for example, contains several intense absorbance maximums in the region between $453 \mathrm{~nm}$ and $665 \mathrm{~nm}$. Refer to Figure 5-13 for a UV/Vis spectrum ${ }^{76}$ of chlorophyll a and b. Also, refer to Figure 5-14 and Figure 5-15 for chemical structures of chlorophyll a and b.

\footnotetext{
${ }^{74} \mathrm{Hsu}$, C. S.; Drinkwater, D., "Gas Chromatography - Mass Spectrometry in the Petroleum Industry", in Current Practice of Gas Chromatography - Mass Spectrometry, W. M. A. Niessen, Ed., Chromatographic Science Series, Vol 86, Dekker: New York, 2001, Chapter 3, pp. 55 - 94.

${ }^{75}$ Lee, Richard. Agents which promote and stabilize water-in-oil emulsions. Spill Science and Technology Bulletin 1999, 5(2), 117-126.

${ }^{76}$ Figure obtained from Wikipedia [Online]. http:/en.wikipedia.org/wiki/File:Chlorophyll_ab_spectra2.PNG (accessed May 1, 2011).
} 
Figure 5-13 Visible spectra of chlorophyll $a$ and $b^{77}$
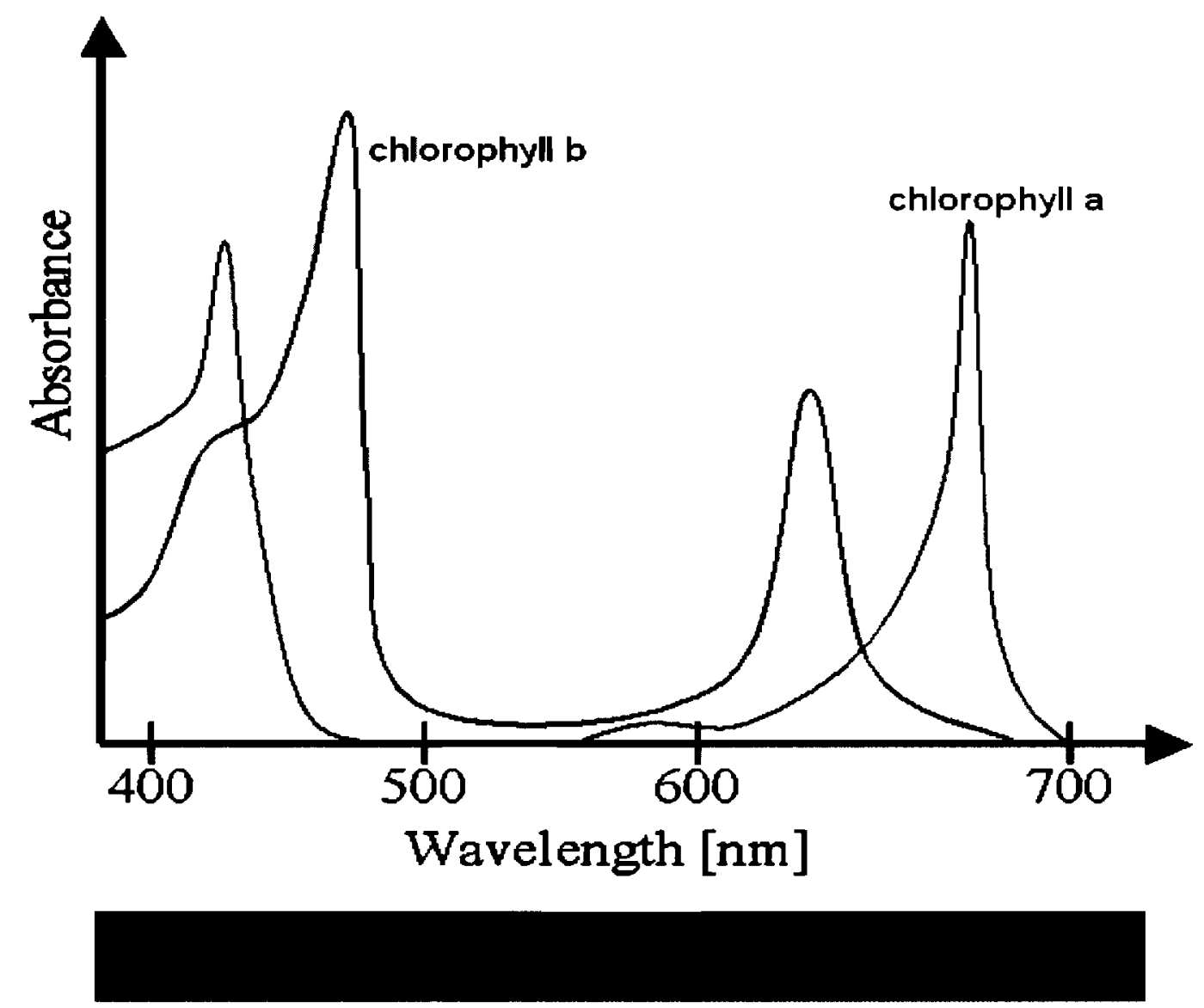

${ }^{77}$ Figure obtained from http://en.wikipedia.org/wiki/File:Chlorophyll_ab_spectra2.PNG (accessed May, 2011). [139] 
Figure 5-14 Chemical structure of chlorophyll a (Chem. Draw)

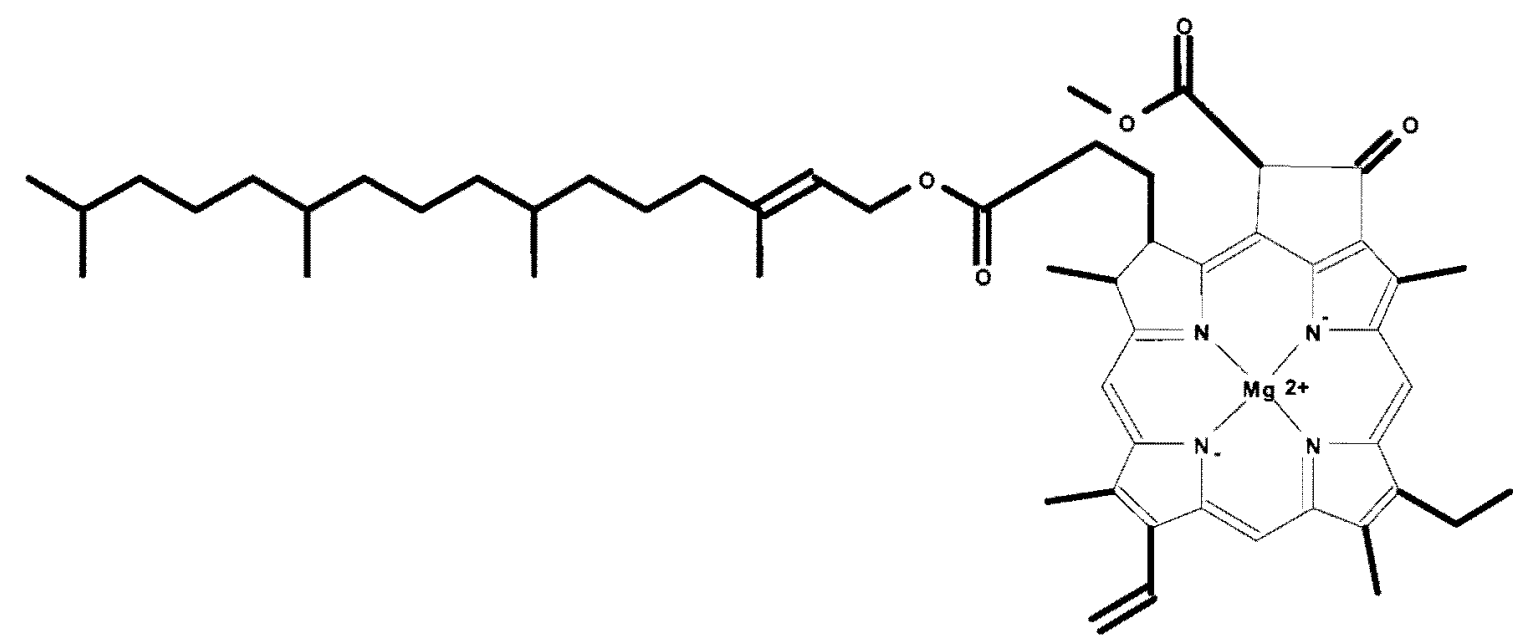

Figure 5-15 Chemical structure of chlorophyll b (Chem. Draw)

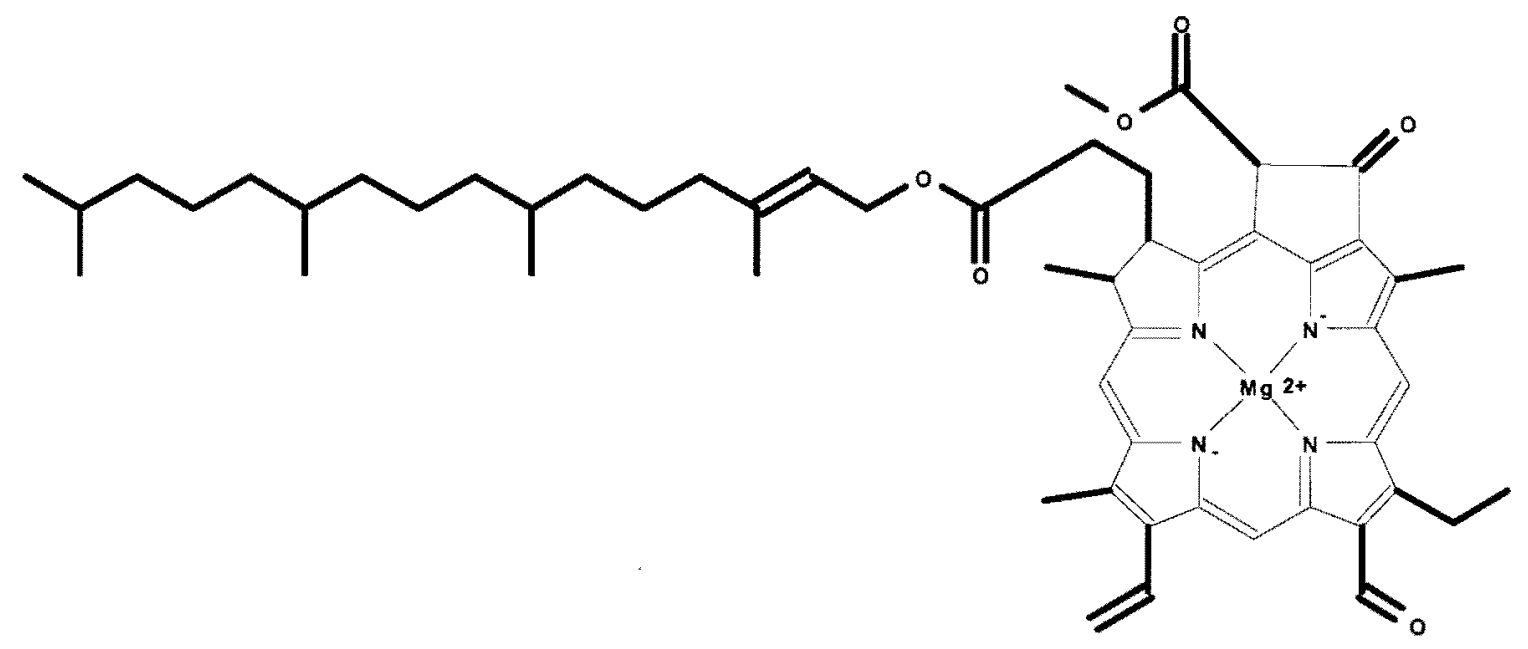


Figure 5-16 UV/Vis spectra of BP oil samples in different solvents: $1 \mathrm{~mm}$ cell: spectrophotometer fully covered from outside lighting

No porphyrin peaks were observed for the oil samples collected. The only absorbance peaks that were detected were associated to interference from the trees and outside light. Refer to the figure below.

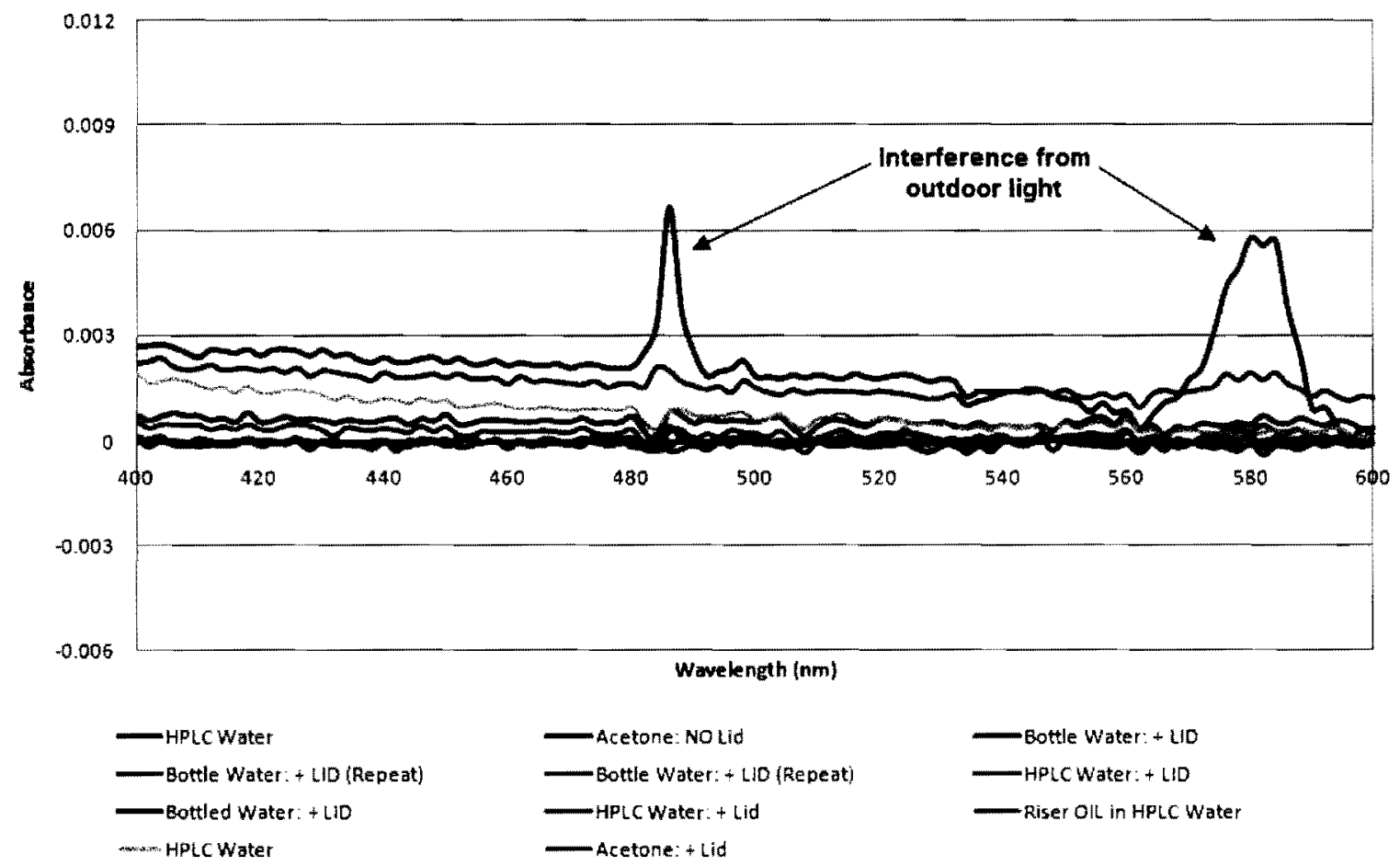




\subsection{Chapter summary}

ATR-IR has been used to simulate the effects of dispersants used to treat a petroleum spill. In response to concerns about the use of dispersants to fight the petroleum spill in the Gulf of Mexico this past summer, we used ATR-IR to investigate how dispersants work. Sample of the BP oil were exposed to $2 \mathrm{wt} \%$ of commercial dishwashing soap. The studies indicate that the hydrocarbon components $\left(\mathrm{CH}_{2}\right.$ bend at $1466 \mathrm{~cm}^{-1}$ and $\mathrm{CH}_{3}$ rock at $\left.1345 \mathrm{~cm}^{-1}\right)$ of the petroleum can be detected in the presence of the soap. However, it took more than $30 \mathrm{~min}$ for it to take effect. This is probably due to time and concentration factors to allow micelles to form. Moreover, this is a creative application of the React-IR concept to study the dynamic behavior of dispersants. Millions of dollars were spent on dispersants to alleviate and eliminate the spread of the BP oil to the natural coast lines. The dispersion experiments demonstrated that even in a controlled and accelerated laboratory environment, it still took several hours for the oil samples to be dispersed. Thus, one begs to question the effectiveness, if any, of the dispersants on the uncontrolled oil spill in the Gulf of Mexico.

Efforts to observe the presence of chlorophyll molecules that are potentially present in the crude oil by UV-Vis spectroscopy were unsuccessful. At this time, the status of this investigation is inconclusive as the presence of these molecules in crude oil is well documented. It is possible that concentrations were below detection limits or the chlorophyll derivatives were insufficiently soluble. Although inconclusive, these investigations provide a foundation for future studies. 


\section{$6 \quad$ Research conclusions}

Dissolution studies are critical tests for measuring the performance of a drug product. In this dissertation, a new technique, in situ attenuated total reflection-infrared spectroscopy (ATRIR), has been developed to monitor dissolutions of pharmaceutical drug formulations. Two over-the-counter (OTC) active drugs (acetaminophen and acetylsalicylic acid) and one calibrator tablet (salicylic acid) were tested during the research. Moreover, both single component and multi-component drug formulations were tested and the individual components were identified by IR spectroscopy. For example, individual components of acetaminophen and acetylsalicylic acid in a caffeine-free formulation of Excedrin $\otimes$ tablets were easily distinguished. The ATR-IR system was found to have very good sensitivity and can analyze samples as low as $0.03 \mathrm{mg} / \mathrm{mL}\left(2 \times 10^{-4} \mathrm{M}\right)$.

In addition, the IR system was able to distinguish separate components of a multiple component system without requiring manual sampling. This versatility was also demonstrated by observing the simultaneous dissolution of acetaminophen and salicylic acid tablets. In contrast, UV-Vis spectroscopy may require mathematical corrections to resolve the two active drugs. Furthermore, HPLC must be used which requires laborious manual sampling or purchase of a complicated automated instrument setup of dissolution configured with HPLC analysis. The in situ ATR-IR method has been validated by comparing dissolution profiles using UV-Vis and HPLC methods. In addition, an accuracy of $\pm 2 \%$ between IR and HPLC for the salicylic acid results was established. With the current configuration of the IR instrument, this analysis is limited by the sensitivity and wavelength range of the in situ fiber 
optic probe. However, since this chapter successfully demonstrates the versatility of this novel application of ATR-IR spectroscopy, it is clear that the method has excellent potential to be improved by modification of the IR instrument and selection of more sensitive probes.

Next, the system has the capability of monitoring drug transformations during dissolution. For instance, the hydrolysis of aspirin to salicylic acid was studied using this technique. The instrument was able to monitor the simultaneous formation and elimination of aspirin while at the same time monitor the formation of salicylic acid in solution. This research was possible because of the versatility of ATR-IR system. Specifically, the unique fingerprint region of the IR spectra gave detailed information about the release profile of the drug. Conventional methods, namely, in-situ UV-Vis, would only show the release profile of one component. However, peak deconvolution software can be used to analyze aspirin hydrolysis. Moreover, the hydrolysis and transformation of the drug from one form to another may be missed with conventional techniques. ATR-IR captures those minor details and displays them in the fingerprint region, as shown earlier in the results. The unique feature of this system is that it is able to detect several forms of the same drug. In other words, it was able to detect the formation and elimination of a pharmaceutical drug (aspirin) while detecting the formation of the hydrosylate of the aspirin (salicylic acid) in real-time. In addition, this system could be used to study other potential transformations. One example might be the dimerization of acetaminophen as mentioned earlier. The novelty with this particular system is its capability to see drug transformations in solutions while conducting the experiment in-situ.

Also, this system was used to support the research related to the BP oil spill. The ATR-IR system coupled with dissolution was able evaluate the effect of dishwashing soaps in the 
dispersion of crude oil and the results call into question the efficacy of application of dispersants in certain situations.

Lastly, a new instrument design was proposed and discussed in this thesis. The proposed system, also known as the Solv-IR, would be built at Seton Hall University. An MRI NSF grant was recently filed for this proposal. The proposal to develop this instrument aims construct a new type of detection technique that will allow the study of solvation of solutes in aqueous and organic systems. ${ }^{787980}$ The proposed unit has great potential for use in studies of dissolution of drugs and monitoring competing reactions such as hydrolysis of pro-drugs in pharmaceutical development, formulation and quality assurance. The proposed instrument also has application in environmental chemistry, especially to study of the fundamental chemistry and remediation of anthropomorphic events. ${ }^{81} 8283$

\footnotetext{
${ }^{78}$ Chen, Zeng-Ping; Morris, Julian; Borissova, Antonia; Khan, Shahid; Mahmud, Tariq; Penchev, Rado; Roberts, Kevin. On-line monitoring of batch cooling crystallization of organic compounds using ATR-FTIR spectroscopy coupled with an advanced calibration method. Chemometrics and Intelligent Laboratory Systems 2009, 96, 49-58.

${ }^{79}$ Wartewig, Siegfried; Neubert, Reinhard. Pharmaceutical applications of Mid-IR and Raman spectroscopy. Advanced Drug Delivery Reviews 2005, 57, 1144-1170.

${ }^{80}$ Kubicki, J.D.; Schroeter, L.M.; Itoh, M.J.; Nguyem, B.N.; Apitz, S.E. Attenuated total reflectance Fouriertransform infrared spectroscopy of carboxylic acids adsorbed onto mineral surfaces. Geochimica et Cosmochimica Acta 1999, 63(18), 2709-2725.

${ }^{81}$ Mas, Silvia; Juan, Anna de; Tauler, Roma; Olivieri, Alejandro; Escandar, Graciela. Application of chemometric methods to environmental analysis of organic pollutants: A review. Talanta 2010, 80, $1052-1067$. ${ }^{82}$ Varela, R.F.; Rodriguez, D.S.; Carracedo, M.P.; Andrade, J.M.; Fernandez, E, Muniategui, S.; Prada, D. Screening the origin and weathering of oil slicks by attenuated total reflectance mid-IR spectrometry. Talanta 2005, 68 116-125.

${ }^{83}$ Varela, R.F.; Carracedo, M.P.; Rivera, P.F.; Andrade, J.M., Muniategui, S.; Prada, D. Monitoring photooxidation of the Prestige's oil spill by attenuated total reflectance infrared spectroscopy. Talanta 2006, 69, 409-417.
} 


\section{$7 \quad$ Appendix [A]: In situ ATR-IR Spectroscopy and Dissolution Data}

The chart below is an example of the digitized data that is obtained for the calibration of

salicylic acid using ATR-IR. The data corresponds to Figure 2-1 in Chapter 2.

Salicylic Acid Linearity Raw Data

\begin{tabular}{|c|c|c|c|c|c|c|c|c|}
\hline & $L$ & $M$ & $N$ & 0 & $P$ & $Q$ & $\mathbf{R}$ & $\mathbf{S}$ \\
\hline$T$ & Wavenumber $(\mathrm{cm}-1)$ & Std-1 & Sid-2 & Std-3 & Std-4 & Std-5 & Std-6 & Std 7 \\
\hline 2 & 1899.4378 & 0.001186 & 0.000446 & 0.000116 & 0.000126 & 0.000327 & 0.000332 & -0.00013 \\
\hline 3 & 1895.7051 & 0.000956 & 0.000358 & $2.97 E-05$ & $6.36 \mathrm{E}-05$ & -0.0001 & $-2.8 E-05$ & 0.00048 \\
\hline 4 & 1891.9744 & 0.001085 & 0.000601 & 0.000275 & $5.67 E-05$ & $-3.7 E-05$ & 0.00021 & 0.00061 \\
\hline 5 & 1888.2427 & 0.00135 & 0.000766 & 0.000266 & 0.000206 & $-8.9 E-05$ & -0.0001 & -0.00035 \\
\hline 6 & 1884511 & 0.001409 & 0.000702 & 0.000398 & B.74E.06 & -0.00021 & 0.000126 & $6.02 \mathrm{E}-05$ \\
\hline 7 & 1880.7793 & 0.001273 & 0.000655 & 0.000511 & 7.17E-06 & -0.00016 & 0.000112 & 0.000106 \\
\hline 8 & 1877.0476 & 0.001176 & 0.000475 & 0.000361 & 0.000187 & $3.85 E-05$ & $-9.1 E-05$ & 0.000104 \\
\hline 9 & 1873.3159 & 0.000934 & 0.000146 & $-3.1 E-05$ & -0.00017 & $5.28 E-05$ & -0.00037 & $5.46 E-05$ \\
\hline 10 & 1869.5842 & 0.000714 & 0.00013 & -0.00018 & -0.00025 & -0.00018 & -0.00035 & -0.00013 \\
\hline 11 & 1865.8525 & 0.000853 & 0.000385 & 0.000114 & 0.000137 & $-8.6 \mathrm{E}-05$ & -7E-05 & -0.00018 \\
\hline 12 & 1862,1208 & 0.000974 & 0.000557 & 0.00033 & 0.000109 & $7.95 E-05$ & $-9.2 E-05$ & -0.00014 \\
\hline 13 & 18583891 & 0.001009 & 0.000404 & 0.000217 & -0.00014 & $-6.7 E-05$ & -0.00033 & 0.00025 \\
\hline 14 & 18546574 & 0.001254 & 0.0000447 & 0.000337 & 0.000101 & 0.000163 & $-9.8 E-05$ & -0.00016 \\
\hline 15 & 1850.9256 & 0.001315 & 0.000639 & 0.00059 & 0.000189 & 0.000494 & 0.00038 & 0.000282 \\
\hline 16 & 1847.1939 & 0.000906 & 0.000532 & 0.000400 & 0.000194 & 0.00038 & 0.000376 & 0.000391 \\
\hline 17 & 1843.4622 & 0.000585 & 0.000351 & $6.54 \mathrm{E}-0.5$ & 0.000176 & $-2.1 E-05$ & -0.00017 & $-4.1 E-05$ \\
\hline 18 & 1839.7305 & 0.000589 & 0.000239 & $7.59 E-05$ & 0.000164 & -0.00012 & -0.00024 & -0.00028 \\
\hline 19 & 1835.9988 & 0.000927 & 0.00026 & 0.0004 & 0.000308 & 0.000295 & 0.000233 & 0.000175 \\
\hline 20 & 1832.2671 & 0.00112 & 0.000365 & 0.000567 & 0.000381 & 0.000366 & 0.000376 & 0.000426 \\
\hline 21 & 1828.5354 & 0.001019 & 0.000457 & 0.000329 & 0.000243 & 0.000125 & 0.000229 & 0.000222 \\
\hline 22 & 18248037 & 0.000767 & 0.000366 & 0.00014 & $7.26 \mathrm{E}-0.5$ & $5.94 E-05$ & -0.00013 & $4.6 F-05$ \\
\hline 23 & 1821.072 & 0.000492 & 0.0001 & $-5 E-0 S$ & 0.00022 & -0.0002 & -0.00044 & -0.00028 \\
\hline 24 & 1817.3403 & 0.000374 & -0.00011 & -0.00023 & -0.00027 & 0.00034 & -0.00043 & -0.0004 \\
\hline 25 & 1813.6086 & 0.000341 & $-4.6 E-05$ & -0.00019 & $-7.1 E-05$ & -0.00019 & -0.0002 & -0.0002 \\
\hline 26 & 1809.8769 & 0.000349 & $-13 E_{-05}$ & -0.0002 & $8 E-06$ & -0.00023 & -0.00015 & 0.00024 \\
\hline 27 & 1806.1452 & 0,000152 & $-59 E-05$ & -0.00019 & $-3.2 E-05$ & -0.00011 & $-9.8 E-05$ & 0.0002 \\
\hline 28 & 1802.4135 & 0.000112 & $.17 E-05$ & -0.00012 & $-8 E-05$ & $5.51 \mathrm{E}-05$ & 1.67E-05 & $-6.1 E-05$ \\
\hline 29 & 1798.6818 & 0.000295 & 0.000208 & $6.25 E-05$ & $5.22 E-05$ & 0.000209 & 0.000276 & 0.000301 \\
\hline 30 & 1794.9501 & 0.000326 & 0.000391 & 0.000215 & 0.000247 & 0.000343 & 0.000482 & 0.000572 \\
\hline 31 & 1791.2184 & 0.000106 & $9.28 E-05$ & $6.89 E-06$ & $-5.5 E-05$ & 8.09E-05 & 5.69E-05 & 0.000151 \\
\hline 32 & 1787.4867 & 0.000117 & $4.11 \mathrm{E} 06$ & $-9.7 E_{-05}$ & -0.0002 & -0.00013 & -0.00017 & $-9.8 E-05$ \\
\hline 33 & 1783.755 & 0.000426 & 0.000234 & 0.00014 & 9.9E-05 & $2.59 \mathrm{~F}-05$ & $-5 E-05$ & $-2.2 E-05$ \\
\hline 34 & 1780.0233 & 0.000404 & 0.000284 & 0.000199 & 0.000391 & 0.000302 & 0.000199 & 0.00021 \\
\hline 35 & 1776.2915 & 0.000371 & 0.000486 & 0.000451 & 0.000764 & 0.000805 & 0.000895 & 0.000987 \\
\hline 36 & 1772.5598 & 0.000245 & 0.000244 & 0.000312 & 0.000401 & 0.000523 & 0.000537 & 0.000742 \\
\hline 37 & 1768.8281 & $3.88 \mathrm{E}-05$ & $-7.4 \mathrm{E}-05$ & $3.94 \mathrm{E}-06$ & -0.00012 & 0.000101 & 0.00011 & 0.00013 \\
\hline 38 & 1765.0964 & $-6.3 E-05$ & $-2.1 E-05$ & 9.21E-06 & -0.00015 & 0.000165 & 0.00013 & 0.000171 \\
\hline 39 & 1761.3647 & -0.00016 & $-2 E-05$ & $-2.3 E-05$ & $-9.5 E-05$ & $6.08 E-05$ & $6.12 \mathrm{E}-05$ & 0.000174 \\
\hline 40 & 1757.633 & 0.00019 & B.03E-05 & $5.82 \mathrm{E}-05$ & 0.000166 & 0.000218 & 0.000284 & 0.000409 \\
\hline 41 & 1753.9013 & -0.00029 & 0.000117 & 0.000191 & 0.000436 & 0.000616 & 0.000827 & 0.000916 \\
\hline 42 & 1750.1696 & -0.00049 & -0.00017 & $9.82 \mathrm{E}-06$ & 9.82E-05 & 0.000345 & 0.000517 & 0.000566 \\
\hline 43 & 1746.4379 & -0.00056 & -0.00027 & -0.00012 & $-1.9 E-05$ & 0.00025 & 0.000287 & 0.000317 \\
\hline 44 & 1742.7062 & -0.00051 & $-8.6 E-05$ & $-3.5 E-05$ & 0.000333 & 0.000621 & 0.000642 & 0.00077 \\
\hline 45 & 1738.9745 & -0.00046 & 0.00021 & 0.000433 & 0.000988 & 0.001429 & 0.001708 & 0.002004 \\
\hline 46 & 1735.2428 & -0.00063 & 0.000101 & 0.000445 & 0.000915 & 0.001478 & 0,001787 & 0.001949 \\
\hline 47 & 1731.5111 & -0.00103 & ${ }^{-0.00055}$ & -0.00038 & -0.00015 & 0.000226 & 0.00019 & 0.000184 \\
\hline 48 & 1727.7794 & -0.0011 & -0.0006 & -0.00057 & -0.00043 & -0.0002 & -0.0003 & -0.00019 \\
\hline 49 & 1724.0477 & -0.00102 & -0.00026 & $-8.8 E-06$ & 0.000193 & 0,000553 & 0.00063 & 0.000899 \\
\hline 50 & 1720.316 & -0.00096 & $3.21 \mathrm{E}-05$ & 0.000646 & 0.00114 & 0.001678 & 0.002053 & 0.002386 \\
\hline 51 & 1716.5843 & -0.00101 & -0.00022 & 0.000232 & 0.000695 & 0.001058 & 0.001399 & 0.00156 \\
\hline
\end{tabular}




\begin{tabular}{|c|c|c|c|c|c|c|c|c|}
\hline & $L$ & $M$ & $\mathrm{~N}$ & 0 & $P$ & $\mathrm{Q}$ & $\mathrm{R}$ & $s$ \\
\hline & 1712.8526 & -0.00135 & -0.00057 & -0.00028 & $-8.4 E-05$ & 0.000218 & 0.000394 & 0.000496 \\
\hline & 1709.1209 & -0.00169 & -0.00061 & -0.0004 & $-8.9 E-05$ & 0.000276 & 0.000494 & 0.000667 \\
\hline 5 & 1705.3892 & -0.00158 & -0.00028 & 0.000105 & 0.000678 & 0.001315 & 0.001761 & 0.002152 \\
\hline 55 & 1701.6574 & -0.00137 & $-6.4 E-05$ & 0.000622 & 0.001256 & 0.002196 & 0.002715 & 0.003221 \\
\hline 56 & 1697.9257 & -0.00151 & -0.00049 & 0.000211 & 0.000605 & 0.001399 & 0.001807 & 0.00208 \\
\hline 57 & 1694.194 & -0.00179 & -0.00087 & -0.00035 & -0.0003 & 0.000253 & 0.00053 & 0.000575 \\
\hline 58 & 1690.4623 & -0.00172 & -0.00044 & 0.000117 & 0.000371 & 0.001154 & 0.001581 & 0.001793 \\
\hline & 1686.7306 & -0.00149 & $6.36 \mathrm{E}-05$ & 0.000642 & 0.001131 & 0.002001 & 0.002561 & 0.002855 \\
\hline 6 & 1682.9989 & -0.00136 & -0.00022 & 0.000184 & 0.000488 & 0.00093 & 0.001237 & 0.001419 \\
\hline & 1679.2672 & -0.00103 & -0.00018 & 0.000147 & 0.000437 & 0.000719 & 0.001093 & 0.0014 \\
\hline 62 & 1675.5355 & -0.00058 & 0.000105 & 0.000503 & 0.000868 & 0.001316 & 0.001638 & 0.001966 \\
\hline $6:$ & 1671.8038 & -0.00026 & 0.00024 & 0.000747 & 0.001202 & 0.001627 & 0.001811 & 0.001981 \\
\hline 64 & 1668.0721 & -0.00024 & 0.000271 & 0.000683 & 0.001076 & 0.001491 & 0.001617 & 0.001777 \\
\hline 6 & 1664.3404 & $-1.8 E-05$ & 0.000317 & 0.000337 & 0.000815 & 0.001329 & 0.001406 & 0.00168 \\
\hline $6 t$ & 1660.6087 & 0.000598 & 0.000614 & 0.000603 & 0.001152 & 0.001739 & 0.002054 & 0.00221 \\
\hline & 1656.877 & 0.001461 & 0.001521 & 0.001783 & 0.002499 & 0.003535 & 0.004215 & 0.004565 \\
\hline $6 t$ & 1653.1453 & 0.002052 & 0.001872 & 0.001991 & 0.002544 & 0.003286 & 0.003881 & 0.00432 \\
\hline 6 & 1649.4136 & 0.002549 & 0.002015 & 0.001953 & 0.002269 & 0.002765 & 0.003057 & 0.003508 \\
\hline 70 & 1645.6819 & 0.002871 & 0.001834 & 0.001371 & 0.001695 & 0.002215 & 0.002284 & 0.00251 \\
\hline 71 & 1641.9502 & 0.003484 & 0.001966 & 0.001583 & 0.001557 & 0.001868 & 0.001993 & 0.002262 \\
\hline 72 & 1638.2185 & 0.004217 & 0.002804 & 0.002488 & 0.002059 & 0.002582 & 0.002784 & 0.003125 \\
\hline 7 & 1634.4868 & 04913 & 0.003363 & 0.002566 & 0.002168 & 0.002763 & 0.0025 & 0.00276 \\
\hline 74 & 1630.7551 & 0.005507 & 0.003671 & 0.002571 & 0.002323 & 0.002625 & 0.002211 & 0.002611 \\
\hline 15 & 1627.0234 & 05408 & 0.003017 & 0.002313 & 0.0019 & 0.002059 & 0.001872 & 0.002161 \\
\hline 76 & 16232916 & 0.005116 & 0.00253 & 0.002077 & 0.001755 & 0.002031 & 0.001956 & 0.002238 \\
\hline & & & 0.002607 & & & & & \\
\hline 78 & 1615.8282 & 0.004013 & 0.002211 & 0.001505 & 0.001531 & 0.001478 & 0.001332 & 0.001496 \\
\hline & 1612.0965 & & 0.0020 & & 1264 & & & 0.001137 \\
\hline 80 & 1608.3648 & 0.003529 & 0.001883 & 0.001704 & 0.001158 & 0.001232 & 0.00126 & 0.001033 \\
\hline & 1604.6331 & & 0.001683 & & 0.000724 & & & 0.00065 \\
\hline 8. & 1600.9014 & 0.003843 & 0.001906 & 0.001293 & 0.000651 & 0.000812 & 0.000723 & 0.0006 \\
\hline & 1597.1697 & & 0.002021 & 0.001197 & 0.000801 & 0.000832 & 0.000642 & 0.000628 \\
\hline 8 & 1593.438 & 0.00446 & 0.002019 & 0.001239 & 0.000974 & 0.000834 & 0.000778 & 0.000709 \\
\hline & 1589.7063 & 379 & 0.002145 & 0.00 & 0.001154 & 0.0 & 1973 & 0.000692 \\
\hline & 1585.9746 & 0.004226 & 0.001989 & 0.001427 & 0.001185 & 0.001046 & 0.000976 & 0.000783 \\
\hline & 1582.2429 & & 0.00 & 0.0 & 0.001804 & 0.0 & 973 & 0.002109 \\
\hline$\overline{8}$ & 1578.51 & & 0.00 & & & & & 0.0 \\
\hline & 72 & & 0.002667 & 0.002121 & 0.001997 & 0.00223 & 0.002352 & 0.002464 \\
\hline 90 & 1571 & & & & 0.001532 & & & 0.001755 \\
\hline & & & 0.00 & 0.00 & 0.001662 & & 0.06 & 0.002042 \\
\hline 9 & 1563.58 & & 0.002 & 0.002 & & & & 0.004606 \\
\hline 9 & 27 & & 0.002666 & 0.002645 & 0.003158 & 0.003769 & 0.004346 & 0.004669 \\
\hline 9 & 1556.121 & & 0.001626 & 0.001474 & & & & 0.002324 \\
\hline $9 !$ & 1552.3893 & 0.001426 & 0.000998 & 0.000854 & 0.000824 & 0.000991 & 0.00114 & 0.001238 \\
\hline$\overline{9 x}$ & 1548.6575 & 0.000831 & 0.000796 & 0.000863 & 0.00107 & 0.001416 & 0.001524 & 0.001795 \\
\hline 97 & & & 0.001292 & & & & & 0.003976 \\
\hline 9 & 1541.1 & 0.000631 & & 0.001695 & & 0.003 & 0.003621 & 0.003955 \\
\hline 9 & 1537.4624 & 1.89E-05 & & & & & 0.001916 & 0.001958 \\
\hline 10 & 1533.7307 & & -8.71 & $6.67 \mathrm{E}-05$ & 0.000373 & 0.000723 & 0.000964 & 0.000992 \\
\hline & 1529.999 & -0.00041 & $1.6 E-05$ & 0.000245 & 0.000508 & 0.001002 & & 0.001 \\
\hline & & & 0.000454 & 0.000897 & 0.001294 & 0.002015 & 0.002362 & 0.002688 \\
\hline
\end{tabular}




\begin{tabular}{|c|c|c|c|c|c|c|c|c|}
\hline & L & $M$ & $N$ & 0 & $P$ & $\mathbf{Q}$ & $\mathbf{R}$ & $\mathbf{S}$ \\
\hline Tos] & 1522.5356 & -0.00044 & 0.000371 & 0.000883 & 0.001318 & 0.001992 & 0.002417 & 0.002748 \\
\hline 104 & 1518.8039 & -0.00067 & -0.00012 & 0.000273 & 0.000559 & 0.001058 & 0.001415 & 0.00154 \\
\hline 105 & 1515.0722 & -0.00072 & -0.00024 & 0.000117 & 0.000303 & 0.000764 & 0.001079 & 0.001165 \\
\hline 106 & 1511.3405 & -0.00043 & 0.000349 & 0.000883 & 0.001316 & 0.002082 & 0.002589 & 0.002924 \\
\hline 107 & 1507.6088 & -0.00021 & 0.000441 & 0.000982 & 0.001496 & 0.002189 & 0.002707 & 0.00305 \\
\hline 108 & 1503.8771 & -0.0001 & 0.000149 & 0.000393 & 0.000684 & 0.00108 & 0.001374 & 0.001435 \\
\hline 109 & 1500.1454 & 0.000916 & 0.000788 & 0.000863 & 0.000905 & 0.001411 & 0.001686 & 0.001733 \\
\hline 110 & 1496.4137 & 0.003357 & 0.001924 & 0.001548 & 0.001223 & 0.001588 & 0.001689 & 0.001733 \\
\hline 111 & 1492.682 & 0.006785 & 0.003629 & 0.002652 & 0.002147 & 0.002244 & 0.002175 & 0.002169 \\
\hline 112 & 1488.9503 & 0.007163 & 0.0037 & 0.002682 & 0.002235 & 0.002164 & 0.001971 & 0.001924 \\
\hline 113 & 1485.2186 & 0.004093 & 0.001979 & 0.001384 & 0.001053 & 0.001048 & 0.000952 & 0.000879 \\
\hline 114 & 1481.4869 & 0.001505 & 0.000668 & 0.000422 & 0.000392 & 0.00055 & 0.000608 & 0.000618 \\
\hline 115 & 1477.7552 & 0.000584 & 0.000497 & 0.000561 & 0.00091 & 0.001281 & 0.001507 & 0.001674 \\
\hline 116 & 1474.0234 & & & 0.000678 & 0.000891 & & 0.001515 & 0.001708 \\
\hline 117 & 1470.2917 & 0651 & 0.000 & 0.000357 & 0.000312 & & 0.000659 & 0.000713 \\
\hline$\frac{118}{119}$ & $\begin{array}{c}1466.56 \\
1462.8283\end{array}$ & $\begin{array}{l}0.002771 \\
0.006074\end{array}$ & $\begin{array}{l}0.001417 \\
0.003346\end{array}$ & $\begin{array}{c}0.001032 \\
0.00261\end{array}$ & $\begin{array}{l}0.001008 \\
0.002558\end{array}$ & & $\begin{array}{l}0.001119 \\
0.002668\end{array}$ & $\begin{array}{c}0.00106 \\
0.002682\end{array}$ \\
\hline 120 & 1459.0966 & 0.006588 & 0.003804 & 0.003064 & 0.003025 & 0.00 & 0.003477 & 0.003606 \\
\hline 121 & 1455.3649 & 853 & 0205 & 0.001519 & 0.001511 & 721 & 0.001769 & 0.001768 \\
\hline 122 & 1451.6332 & 528 & 0.00068 & 0.000414 & 0.000513 & 0.000609 & 0.000658 & 0.000649 \\
\hline 123 & 1447.9015 & 08 & -0.0 & -0.00019 & -0.00013 & & -05 & 1.82E-05 \\
\hline 124 & 1444.1698 & -0.00029 & -0.00043 & -0.00044 & -0.00056 & 048 & -0.00034 & -0.00034 \\
\hline 125 & 1440.4381 & 025 & -0.0 & $-7 E-05$ & 0018 & -05 & 275 & 0.000414 \\
\hline 126 & 1436.7064 & 035 & 0021 & $-3.9 E-05$ & .05 & 0.0 & 341 & 0.000446 \\
\hline 127 & 1432.9747 & -0.00039 & 0002 & -0.00015 & $-4 E-05$ & -05 & 0016 & 0.000179 \\
\hline 128 & 1429.243 & -0.0 & 12 & $-5.3 \mathrm{E}-05$ & 113 & & 237 & 1254 \\
\hline 129 & 1425.5113 & -0.00023 & $-5.6 \mathrm{E}-05$ & $721 \mathrm{E}-05$ & 0.000328 & 0.00 & 00585 & 0.000652 \\
\hline 130 & 1421.7796 & 05 & 0.0 & 0361 & 0549 & 0.0 & 073 & 0.001228 \\
\hline 131 & 1418.0479 & 0.000413 & 0.000187 & 0.000263 & 0.000111 & 0443 & 10471 & 0.000569 \\
\hline 132 & 1414.3162 & 0.000783 & 0.000316 & 0.000287 & $5.03 \mathrm{k}$ & 194 & 0178 & 0.000115 \\
\hline 133 & 1410.5845 & 0.001229 & 0.000692 & 0.000593 & 0.000 & 466 & 553 & 0.000449 \\
\hline 134 & 14068528 & 582 & 0.000 & 0738 & 0.0 & 584 & 0.000648 & 0.00062 \\
\hline 135 & 14031211 & 0.00238 & 0.001332 & 0.001071 & 0.000714 & 883 & 0.00092 & 0.000919 \\
\hline 136 & 1399.3894 & 0.00411 & 0.00226 & 0.001745 & 0.001243 & 0.001327 & 0.001341 & 0.001324 \\
\hline 137 & 1395.6576 & 0.00723 & 0.003786 & 0.002654 & 0.001951 & 1754 & 0.001637 & 0.001532 \\
\hline 138 & 1391.9259 & 0.010625 & 0.005419 & 0.003711 & 0.00305 & 0.002591 & 0.002352 & 0.002151 \\
\hline 139 & 1388.1942 & 0.011517 & 0.005803 & 0.003926 & 0.003387 & 0.002882 & 0.002586 & 0.002351 \\
\hline 140 & 1384.4625 & 0.009322 & & 0.002895 & 0.002381 & & 0.001706 & 0.001443 \\
\hline 141 & 1380.7308 & 0.006639 & 0.003193 & 0.002056 & 0.001647 & 0.001377 & 0.00123 & 0.000992 \\
\hline 142 & 1376.9991 & 0.004745 & 0.002303 & 0.001547 & 0.001224 & 0.00108 & 0.000948 & 0.000799 \\
\hline 143 & 1373.2674 & 0.003576 & 0.001644 & 0.001055 & 0.0007 & 0.00067 & 0.000572 & 0.000445 \\
\hline 144 & 1369.5357 & 0.002964 & 0.00128 & 0.00069 & 0.000491 & 0.000432 & 0.000437 & 0.000326 \\
\hline 145 & 1365.804 & 0.002925 & 0.001388 & 0.000801 & 0.000781 & 0.000661 & 0.000687 & 0.000673 \\
\hline 146 & 1362.0723 & 0.00319 & 0.0 & 0.001039 & 0.000897 & 0775 & 0.000748 & 0.000727 \\
\hline 147 & 1358.3406 & 0.003132 & 0.001462 & 0.000881 & 0.000669 & 0.000565 & 0.000511 & 0.00045 \\
\hline 148 & 1354.6089 & 0.0 & 0.001511 & 0.000896 & 0.000839 & 0.000704 & 0643 & 0.000525 \\
\hline 149 & 1350.8772 & 0.0 & 0.0020 & 0.001364 & & & 0.000987 & 0.00081 \\
\hline 150 & 1347.1455 & 0.004446 & 0.002376 & 0.001719 & 0.001276 & 0.001274 & 0.001134 & 0.001006 \\
\hline 151 & 1343.4138 & & & 0.001752 & & & & 0.001173 \\
\hline 152 & 1339.6821 & 0.004391 & 0.002383 & 0.001779 & 0.00129 & 0.001206 & 0.001142 & 0.001097 \\
\hline 153 & 1335.9504 & 0.003976 & 0.002084 & 0.001469 & 0.000776 & 0.000744 & 0.000645 & 0.000552 \\
\hline
\end{tabular}




\begin{tabular}{|c|c|c|c|c|c|c|c|c|}
\hline & L & M & $\mathbf{N}$ & 0 & P & Q & $\mathbf{R}$ & $\mathrm{S}$ \\
\hline 154 & 1332.2187 & 0.00338 & 0.001653 & 0.001025 & 0.000664 & 0.000468 & 0.000352 & 0.000257 \\
\hline 155 & 1328.487 & 0.002996 & 0.001477 & 0.000908 & 0.000941 & 0.000618 & 0.000534 & 0.000424 \\
\hline 156 & 1324.7553 & 0.002511 & 0.00119 & 0.000724 & 0.000769 & 0.000566 & 0.000513 & 0.00043 \\
\hline 157 & 1321.0235 & 0.002244 & 0.001047 & 0.000652 & 0.00052 & 0.000495 & 0.000449 & 0.000424 \\
\hline 158 & 13172918 & 0.002288 & 0.001121 & 0.000739 & 0.000519 & 0.000533 & 0.000496 & 0.000473 \\
\hline 159 & 1313.5601 & 0.002267 & 0.001008 & 0.000576 & 0.000396 & 0.000379 & 0.000356 & 0.000295 \\
\hline 160 & 1309.8284 & 0.002465 & 0.001017 & 0.000567 & 0.000432 & 0.000423 & 0.000394 & 0.000312 \\
\hline 16 & 1306.0967 & 0.002911 & 0.001308 & 0.000842 & 0.000661 & 0.000678 & 0.000606 & 0.000501 \\
\hline 16 & 1302.365 & 0.003314 & 0.001634 & 0.001101 & 0.000778 & & 0.000 & 0.000505 \\
\hline \begin{tabular}{|l|}
163 \\
164 \\
\end{tabular} & $\begin{array}{l}1298.6333 \\
1294.9016\end{array}$ & $\begin{array}{l}0.003299 \\
0.003022\end{array}$ & $\begin{array}{l}0.001693 \\
0.001543\end{array}$ & $\begin{array}{c}0.00116 \\
0.001068\end{array}$ & $\begin{array}{l}0.000854 \\
0.000843\end{array}$ & $\begin{array}{l}0.000696 \\
0.000789\end{array}$ & $\begin{array}{l}0.000622 \\
0.000736\end{array}$ & $\begin{array}{l}0.000489 \\
0.000598\end{array}$ \\
\hline 165 & 1291.1699 & 0.002832 & 0.001469 & 0.001037 & 0.000822 & 0.000878 & 0.000835 & 0.000737 \\
\hline 166 & 1287.4382 & 0.002753 & 0.001452 & 0.001042 & 0.000799 & 0.00074 & 0.000701 & 0.0006 \\
\hline 167 & 1283.7065 & 0.002886 & 0.001571 & 0.001151 & 0.000807 & 0.000676 & 0.00062 & 0.000497 \\
\hline 168 & 1279.9748 & 0.00303 & 0.001679 & 0.00123 & 0.000866 & 0.000823 & 0.000751 & 0.000679 \\
\hline 169 & 1276.2431 & 0.00309 & 0.001643 & 0.001152 & 0.000976 & 0.000917 & 0.00085 & 0.000804 \\
\hline 170 & 1272.5114 & 0324 & 0.001658 & 0.0011 & 0.000864 & 0.000866 & 0.000768 & 0.000716 \\
\hline 171 & 1268.7797 & 0.003783 & 0.00191 & 0.0012 & 0.000783 & 0.000866 & 0.000731 & 0.00064 \\
\hline 172 & 1265.048 & 0.004695 & 0.002387 & 0.001656 & 0.001061 & 0.00095 & 0.000795 & 0.000701 \\
\hline 173 & 1261.3163 & 0.005823 & 0.002982 & 0.002023 & 0.001436 & 1121 & 0.000989 & 0.000863 \\
\hline 174 & 1257.5846 & 0.006738 & 0.003 & 0.00223 & 0.001667 & 343 & 0.001195 & 0.00103 \\
\hline 175 & 1253.8529 & 0.006747 & 0.00 & 0.002168 & 0.001566 & 244 & 0.001031 & 0.000874 \\
\hline 176 & 1250.1212 & 0.00 & 0.00 & 0.0 & 0.001398 & 88 & 919 & 0.000748 \\
\hline 177 & 1246.3894 & 0.0 & 0.0 & 0.0 & 0.001029 & 316 & 0.000 & 0.000495 \\
\hline 178 & 1242.6577 & 0.00 & 0.0 & 1394 & 0.000901 & 741 & 0.00 & 0.00045 \\
\hline 179 & 1238.926 & & & & & & & 548 \\
\hline 180 & 1235.1943 & 0.00 & 0.0 & 1124 & 0.000674 & 462 & 0.00 & 0.000261 \\
\hline 181 & 1231.4626 & 0.0 & 0.00 & 1095 & 0.000713 & 472 & 0.000 & 0.000316 \\
\hline 182 & 1227.7309 & 0.003853 & 0.00 & 0.001355 & 0.001022 & 876 & 0.000 & 0.000738 \\
\hline 183 & 1223.9992 & & 0.001951 & & 0.001098 & & 0.000925 & 0.000854 \\
\hline 184 & 1220.2675 & 0.003658 & 0.001736 & 0.00111 & 0.000889 & 0776 & 0.000738 & 0.000635 \\
\hline 185 & 1216.5358 & 0.003675 & 0.00178 & 0.001127 & 0.000979 & 0.0 & 0.000 & 0.000679 \\
\hline 186 & 1212.8041 & 0.003753 & 0.001899 & 0.001217 & 0.001156 & & 0.000878 & 0.000802 \\
\hline 187 & 1209.0724 & 0.003834 & 0.001958 & 0.001254 & 0.001085 & & 0.000814 & 0.000702 \\
\hline 188 & 1205.3407 & 0.003864 & 0.001879 & 0.001173 & 0.000842 & & 0.000667 & 0.00056 \\
\hline 189 & 1201.609 & 0.00397 & 0.0 & 0.001212 & 0.000742 & 0.000797 & 0.000721 & 0.000614 \\
\hline 190 & 1197.8773 & 0.00 & 0.002 & 0.001457 & 0.000872 & 993 & 0.000873 & 0.000745 \\
\hline 191 & 1194.1456 & & & & & & 0.000 & 0.00077 \\
\hline 192 & 1190.4139 & 976 & 0.00 & 0.00 & 0.001307 & & 908 & 0.000793 \\
\hline 193 & 36.6822 & & & & & & & .000827 \\
\hline 194 & 1182.9505 & 0.00 & 0.00 & 0.002031 & 0.001388 & 0.0 & 0.001153 & 0.001049 \\
\hline 195 & 1179.2188 & 0.006234 & 0.003232 & 0.002276 & 0.001674 & & 0.001306 & 0.001194 \\
\hline 196 & 1175.4871 & 0.006604 & 0.003391 & 0.002266 & 0.001499 & 0.001286 & 0.001087 & 0.000922 \\
\hline 197 & 1171.7553 & 0.006879 & 0.003437 & 0.00217 & 0.001422 & 0.001196 & 0.000999 & 0.000803 \\
\hline 198 & 1168.0236 & 0.007416 & 0.003675 & 0.00235 & 0.001609 & 0.001364 & 0.001155 & 0.000954 \\
\hline 199 & 1164.2919 & 0.008204 & 0.00413 & 0.002754 & 0.00195 & 0.001723 & 0.001427 & 0.00124 \\
\hline 200 & 1160.5602 & 0.008411 & 0.004226 & 0.002832 & 0.002038 & 0.001825 & 0.001501 & 0.001325 \\
\hline 201 & 1156.8285 & 0.007971 & 0.003972 & 0.002644 & 0.001869 & 0.001626 & 0.00136 & 0.001185 \\
\hline 202 & 1153.0968 & 0.007722 & 0.003877 & 0.002546 & 0.001929 & 0.001639 & 0.001426 & 0.001268 \\
\hline 203 & 1149.3651 & 0.007602 & 0.003817 & 0.002446 & 0.001717 & 0.001408 & 0.001198 & 0.001026 \\
\hline 204 & 1145.6334 & 0.006491 & 0.003212 & 0.002016 & 0.001224 & 0.000872 & 0.000653 & 0.000515 \\
\hline
\end{tabular}




\begin{tabular}{|c|c|c|c|c|c|c|c|c|}
\hline & $L$ & $M$ & $\mathbf{N}$ & 0 & $\mathrm{P}$ & Q & $\mathbf{R}$ & $\mathrm{S}$ \\
\hline 205 & 1141.9017 & 0.004839 & 0.002328 & 0.001445 & 0.000891 & 0.000557 & 0.000403 & 0.000323 \\
\hline 206 & 1138.17 & 0.003536 & 0.001676 & 0.001013 & 0.000684 & 0.000464 & 0.000377 & 0.000304 \\
\hline 207 & 1134.4383 & 0.002521 & 0.001262 & 0.000793 & 0.000473 & 0.000403 & 0.000311 & 0.000272 \\
\hline 208 & 1130.7066 & 0.001495 & 0.000742 & 0.000469 & $5.25 E-05$ & $9.44 E-05$ & $-2 \mathrm{E}-05$ & $-1.9 E-05$ \\
\hline 209 & 1126.9749 & 2.45E-05 & -0.0002 & -0.00031 & -0.00054 & -0.00044 & -0.00054 & -0.00052 \\
\hline 210 & 1123.2432 & -0.00118 & -0.00078 & -0.00068 & -0.00059 & -0.00044 & -0.00044 & -0.00041 \\
\hline 211 & 1119.5115 & -0.00226 & -0.00121 & -0.00089 & -0.00069 & -0.00053 & -0.00043 & -0.00039 \\
\hline 212 & 1115.7798 & -0.00371 & -0.00194 & -0.00142 & -0.00106 & -0.00092 & -0.00079 & -0.00075 \\
\hline 213 & 1112.0481 & -0.00512 & -0.00253 & -0.00174 & -0.00144 & -0.00118 & -0.00098 & -0.00095 \\
\hline 214 & 1108.3164 & -0.0066 & -0.00314 & -0.00199 & -0.00193 & -0.00147 & -0.00121 & -0.00104 \\
\hline 215 & 1104.5847 & -0.00868 & -0.00424 & -0.00274 & 0242 & -0.0019 & -0.00161 & -0.00133 \\
\hline 216 & 1100.853 & -0.0111 & -0.00558 & -0.00374 & -0.00309 & -0.00247 & -0.00212 & -0.00187 \\
\hline 217 & 1097.1213 & -0.01326 & -0.00669 & -0.00451 & & -0.00277 & -0.00233 & -0.00204 \\
\hline 218 & 10933895 & -0.01488 & & -0.00519 & -0.00399 & & -0.00268 & -0.00232 \\
\hline 219 & 1089.6578 & -0.01538 & -0.00803 & -0.0055 & -0.00442 & -0.00361 & -0.00308 & -0.00269 \\
\hline 22 & 1085.9261 & -0.01451 & 1762 & -0.00525 & & & -0.00299 & -0.00261 \\
\hline 221 & 1082.1944 & -0.01279 & 678 & -0.00468 & 383 & & 256 & -0.00222 \\
\hline 222 & 1078.4627 & -0.01176 & -0.00634 & -0.00441 & 035 & 274 & -0.00 & -0.00207 \\
\hline 223 & 1074.731 & -0.01193 & & -0.00445 & 348 & 268 & 229 & -0.00198 \\
\hline 22 & 1070.9993 & -0.01227 & -0.0 & -0.00 & -0.00335 & 253 & -0.00214 & -0.00188 \\
\hline & 1067.2676 & -0.0 & -0.0 & -0.00 & & 66 & 229 & -0.00205 \\
\hline 226 & 1063.5359 & -0.01 & -0.0 & -0.00414 & 1336 & 272 & -0.00233 & -0.00211 \\
\hline 227 & 1059.8042 & 61 & -0. & -0.0 & & & 203 & -0.00184 \\
\hline 22 & 1056.0725 & & -0 . & & 23 & & 157 & -0.00139 \\
\hline 229 & 1052.3408 & -0.0 & -0.0 & -0.0 & 161 & 142 & 116 & -0.00099 \\
\hline 23 & 1048.6091 & -0.0 & & & & & & -0.00098 \\
\hline 23 & 1044.8774 & -0.00415 & -0.0 & -0.00 & 134 & 15 & 099 & -0.00085 \\
\hline & 1041.1457 & -0.00 & 138 & -0.0 & 1086 & 057 & -0.00048 & -0.00038 \\
\hline 233 & 1037.414 & -0.0 & -0.0 & -0.0 & & & 039 & -0.00029 \\
\hline & 1033.6823 & & 082 & -0.00 & 62 & & & -0.0004 \\
\hline 235 & 1029.9506 & -0.00 & -0.0 & & & & 056 & -0.0005 \\
\hline & 1026.2189 & -0.00176 & 099 & -0.00075 & -0.00076 & -0.00054 & -0.0005 & -0.00046 \\
\hline 237 & 1022.4872 & -0.00198 & -0.0 & -0.00101 & -0.00087 & & 064 & -0.00064 \\
\hline 238 & 1018.7554 & -0.00221 & -0.00136 & -0.00118 & -0.00104 & & -0.00083 & -0.00087 \\
\hline 239 & 1015.0237 & -0.00208 & -0.00112 & -0.0009 & -0.00069 & & & -0.00051 \\
\hline 240 & 1011.292 & -0.00219 & & -0.00079 & -0.00058 & & & -0.00024 \\
\hline 241 & 1007.5603 & -0.0027 & -0.00139 & -0.00093 & -0.00095 & 074 & -0.00064 & -0.00055 \\
\hline 24 & 1003.8286 & -0.00371 & -0.00185 & -0.00 & -0.00121 & & -0.00075 & -0.00065 \\
\hline 243 & 1000.0969 & -0.00586 & -0.00293 & -0.00 & -0.00154 & 098 & -0.00075 & -0.00062 \\
\hline 244 & 996.3652 & -0.0087 & & -0.00298 & -0.00219 & & -0.00 & -0.00104 \\
\hline 24 & 992.6335 & -0.01 & & -0.00367 & -0.00278 & -0.00222 & -0.00173 & -0.00152 \\
\hline & 988.9018 & & & & & & & -0.0013 \\
\hline 24 & 985.1701 & -0.00724 & -0.0 & -0.00 & & & -0.00087 & -0.00067 \\
\hline 24 & 981.4384 & 489 & & -0.00178 & 0125 & & -0.00071 & -0.00055 \\
\hline 24 & 977.7067 & -0.00331 & -0.0018 & -0.00132 & -0.00096 & -0.00073 & -0.00062 & -0.00049 \\
\hline & 973.975 & & & -0.00079 & -0.00048 & & -0.00021 & -0.0001 \\
\hline & 970.2433 & & & & -0.00039 & & $-9.2 E-05$ & $-5.6 E-05$ \\
\hline 25 & 966.5116 & -0.00044 & -0.00028 & -0.00026 & -0.00024 & & 7.42E-05 & $3.01 E-05$ \\
\hline 25 & 962.7799 & 0.000815 & 0.000539 & 0.00047 & 0.000491 & 0.000655 & 0.000717 & 0.000705 \\
\hline 25 & 959,0482 & 0.002108 & 0.001349 & 0.001195 & 0.001016 & 0.001059 & 0.001048 & 0.0011 \\
\hline & 955,3165 & 0.002958 & 0.001739 & 0.001411 & 0.000979 & 0.000847 & 0.000858 & 0.00087 \\
\hline
\end{tabular}




\begin{tabular}{|c|c|c|c|c|c|c|c|c|}
\hline & L & $M$ & $N$ & 0 & $\mathbf{P}$ & Q & $\mathbf{R}$ & $\mathbf{S}$ \\
\hline 256 & 951,5848 & 0.003795 & 0.002052 & 0.001546 & 0.000769 & 0.000633 & 0.00059 & 0.000539 \\
\hline 257 & 947.8531 & 0.004571 & 0.00237 & 0.001777 & 0.001007 & 0.000903 & 0.000774 & 0.000714 \\
\hline 258 & 944,1213 & 0.004691 & 0.002306 & 0.001617 & 0.001273 & 0.001165 & 0.001099 & 0.000984 \\
\hline 259 & 940.3896 & 0.004372 & 0.002068 & 0.001318 & 0.001026 & 0.000997 & 0.000965 & 0.000868 \\
\hline 260 & 936.6579 & 0.004143 & 0.002079 & 0.001323 & 0.000962 & 0.000931 & 0.000916 & 0.000862 \\
\hline 261 & 932.9262 & 0.003803 & 0.002096 & 0.001401 & 0.001324 & 0.00126 & 0.001301 & 0.001234 \\
\hline 262 & 929.1945 & 0.003135 & 0.001903 & 0.001382 & 0.001508 & 0.001561 & 0.001594 & 0.001528 \\
\hline 263 & 925.4628 & 0.002265 & 0.00147 & 0.001244 & 0.001512 & 0.001764 & 0.001758 & 0.001767 \\
\hline 264 & 921.7311 & 0.001181 & 0.000692 & 0.000776 & 0.001116 & 0.001422 & 0.001473 & 0.001564 \\
\hline 265 & 917,9994 & 0.000139 & 7.48E-05 & 0.000247 & 0.000604 & 0.000756 & 0.000895 & 0.000993 \\
\hline 266 & 914.2677 & -0.00066 & -0.00037 & -0.0001 & 0.00043 & 0.00058 & 0.00072 & 0.000773 \\
\hline 267 & 910.536 & -0.00111 & -0.00055 & -0.00018 & 0.000233 & 0.000703 & 0.000826 & 0.000854 \\
\hline 268 & 906.8043 & -0.00145 & -0.00048 & -0.00016 & 0.000153 & 0.000723 & 0.000877 & 0.000917 \\
\hline 269 & 903.0726 & -0.00203 & -0.00074 & -0.00027 & 0.000119 & 0.000555 & 0.000717 & 0.000854 \\
\hline 270 & 899.3409 & -0.00255 & -0.00114 & -0.00047 & 6.33E-05 & 0.000483 & 0.000604 & 0.000875 \\
\hline 271 & 895.6092 & -0.00298 & -0.00149 & -0.00071 & 5.53E-05 & 0.000489 & 0.000728 & 0.000813 \\
\hline 272 & 891.8775 & -0.00341 & -0.00167 & -0.00085 & -0.0003 & 0.000225 & 0.000674 & 0.000554 \\
\hline$\frac{273}{274}$ & $\begin{array}{l}888.1458 \\
884.4141\end{array}$ & $\begin{array}{l}-0.00366 \\
-0.00371\end{array}$ & $\begin{array}{l}-0.00178 \\
-0.00176\end{array}$ & $\begin{array}{l}-0.00088 \\
-0.00093\end{array}$ & $\begin{array}{c}-0.0005 \\
-0.00054\end{array}$ & $\begin{array}{c}0.000303 \\
0.00054\end{array}$ & $\begin{array}{l}0.000795 \\
0.001018\end{array}$ & $\begin{array}{l}0.000705 \\
0.000964\end{array}$ \\
\hline 275 & 880.6824 & -0.00388 & -0.00205 & -0.00119 & -0.00095 & $271 \mathrm{E}-05$ & 0.000539 & 0.00056 \\
\hline 276 & 876.9507 & -0.00393 & -0.00226 & -0.00116 & -0.00079 & $-7 E-05$ & 0.000362 & 0.000589 \\
\hline 277 & 873219 & -0.00433 & -0.00239 & -0.00119 & -0.00055 & 0.000101 & 0.00032 & 0.000496 \\
\hline 278 & 869.4872 & -0.00487 & -0.00264 & -0.00157 & -0.0008 & $-8 E-05$ & 0.000167 & 0.000311 \\
\hline 279 & 865.7555 & -0.00509 & -0.00283 & -0.00172 & -0.00128 & -0.0004 & -0.00018 & 0.000181 \\
\hline 280 & 862.0238 & -0.00537 & -0.00265 & -0.00138 & -0.00101 & -0.00023 & 0.000242 & 0.000488 \\
\hline 281 & 858.2921 & -0.00619 & -0.00314 & -0.00166 & -0.00095 & -0.00016 & 0.000614 & 0.000697 \\
\hline 282 & 854.5604 & -0.00725 & -0.00408 & -0.00256 & -0.00151 & -0.00072 & -0.00019 & 0.000125 \\
\hline 283 & 850.8287 & -0.00788 & -0.00415 & -0.00262 & -0.00135 & -0.00058 & -0.0001 & 0.000241 \\
\hline 284 & 847.097 & 0.00796 & -0.0037 & -0.00195 & -0.0009 & 0.00016 & 0.000811 & 0.000701 \\
\hline 285 & 843.3653 & -0.00754 & -0.00338 & -0.00168 & -0.00072 & 0.000331 & 0.000964 & 0.001029 \\
\hline$\frac{286}{287}$ & $\begin{array}{l}839.6336 \\
835.9019\end{array}$ & $\begin{array}{c}-0.0077 \\
-0.00812\end{array}$ & $\begin{array}{l}-0.00362 \\
-0.00387\end{array}$ & $\begin{array}{l}-0.00202 \\
-0.00244\end{array}$ & $\begin{array}{l}-0.00133 \\
-0.00186\end{array}$ & $\begin{array}{l}-0.00016 \\
-0.00039\end{array}$ & $\begin{array}{c}0.00029 \\
9.25 E-05\end{array}$ & $\begin{array}{l}0.000708 \\
0.000235\end{array}$ \\
\hline 268 & 832.1702 & -0.00763 & -0.00413 & -0.00235 & -0.00145 & 1.14E-05 & 0.000779 & 0.000564 \\
\hline 289 & 828.4385 & -0.00704 & -0.00427 & -0.00174 & -0.00094 & 0.000403 & 0.001395 & 0.001235 \\
\hline 290 & 824.7068 & -0.0077 & -0.00418 & -0.00212 & -0.00147 & 0.000107 & 0.000949 & 0.000877 \\
\hline 291 & 820.9751 & -0.0082 & -0.00388 & -0.00268 & -0.00202 & -0.00054 & -0.0003 & -0.00029 \\
\hline 292 & 817.2434 & -0.00781 & -0.00401 & -0.00245 & -0.00199 & -0.00124 & -0.00118 & -0.00075 \\
\hline 293 & 813.5117 & -0.00678 & -0.00338 & -0.00158 & -0.0009 & -0.00054 & 0.000213 & 0.000371 \\
\hline 294 & 809.78 & -0.00636 & -0.00321 & -0.00153 & -0.00048 & $3.4 E-05$ & 0.00109 & 0.000918 \\
\hline 295 & 806.0483 & -0.00736 & -0.00368 & -0.00201 & -0.00164 & -0.0004 & 0.000307 & 0.000387 \\
\hline$\frac{296}{297}$ & $\begin{array}{l}802.3166 \\
798.5849\end{array}$ & $\begin{array}{l}-0.00797 \\
-0.00819\end{array}$ & $\begin{array}{r}-0.00406 \\
-0.00484\end{array}$ & $\begin{array}{r}-0.00254 \\
-0.00311\end{array}$ & $\begin{array}{l}-0.00259 \\
-0.00241\end{array}$ & $\begin{array}{r}-0.00083 \\
-0.00127\end{array}$ & -0.00051 & 0.000109 \\
\hline 298 & 794.8532 & -0.0082 & -0.0049 & -0.00359 & -0.00217 & -0.00133 & $-0,00064$ & $-4.4 E-05$ \\
\hline 299 & 791.1214 & -0.00786 & -0.00405 & -0.00322 & -0.00221 & -0.00135 & $-4.8 E-05$ & 0.000357 \\
\hline 300 & 787.3897 & -0.00714 & -0.00339 & -0.00148 & -0.00058 & $1.62 \mathrm{E}-05$ & 0.000994 & 0.001373 \\
\hline 301 & 783.658 & -0.00734 & -0.00333 & -0.001 & 0.000207 & 0.000315 & 0.001158 & 0.0018 \\
\hline 302 & 779.9263 & -0.00693 & -0.00292 & -0.00114 & -0.00023 & $-1 E-04$ & 0.000946 & 0.001363 \\
\hline 303 & 776.1946 & -0.00613 & -0.00272 & -0.00141 & -0.00076 & 0.000415 & 0.001108 & 0.001337 \\
\hline 304 & 772.4629 & -0.00777 & -0.00383 & -0.00335 & -0.00201 & $-7.5 E-05$ & 0.000259 & 0.000769 \\
\hline 305 & 768.7312 & -0.00763 & -0.00403 & -0.00417 & -0.00264 & -0.00036 & -0.00019 & 0.00045 \\
\hline 306 & 764.9995 & -0.00575 & -0.00161 & -0.0015 & -0.00051 & $-6.5 \mathrm{E}-05$ & 0.001896 & 0.00175 \\
\hline
\end{tabular}


Salicylic Acid Linearity Raw Data

\begin{tabular}{|c|c|c|c|c|c|c|c|c|}
\hline & $L$ & $M$ & $N$ & 0 & $P$ & Q & $\mathbf{R}$ & $\mathrm{S}$ \\
\hline 307 & 761.2678 & -0.00503 & -0.00106 & -0.00032 & 0.001092 & $5.23 E-05$ & 0.00358 & 0.001615 \\
\hline$\frac{308}{309}$ & $\begin{array}{l}757.5361 \\
753.8044\end{array}$ & $\begin{array}{r}-0.00629 \\
0.00683\end{array}$ & $\begin{array}{l}-0.00331 \\
-0.00424\end{array}$ & $\begin{array}{l}-0.0015 \\
-0.0015\end{array}$ & $\begin{array}{l}-0.00044 \\
-0.00113\end{array}$ & $\begin{array}{r}-0.00015 \\
0.000211\end{array}$ & $\begin{array}{l}0.003362 \\
0.002343\end{array}$ & $\begin{array}{l}0.001435 \\
0.003148\end{array}$ \\
\hline \begin{tabular}{|l|}
310 \\
311
\end{tabular} & $\begin{array}{c}750.0727 \\
746.341\end{array}$ & $\begin{array}{l}-0.00725 \\
-0.00736\end{array}$ & $\begin{array}{l}-0.00467 \\
-0.00409\end{array}$ & $\begin{array}{l}-0.00072 \\
-0.00072\end{array}$ & $\begin{array}{l}-0.00203 \\
-0.00209\end{array}$ & $\begin{array}{l}0.000186 \\
0.000299\end{array}$ & $\begin{array}{l}0.000415 \\
0.000565\end{array}$ & $\begin{array}{l}0.003189 \\
0.001658\end{array}$ \\
\hline 312 & 742.6093 & -0.00727 & -0.0023 & -0.00152 & -0.00068 & 0.000539 & 0.00208 & 0.001731 \\
\hline 313 & 738.8776 & .0 .00746 & -0.00387 & -0.00284 & -0.0003 & 0.000658 & 0.002007 & 0.001217 \\
\hline 314 & 735.1459 & -0.00653 & -0.00268 & -0.00376 & 0.000387 & 0.001801 & 0.002261 & 0.002028 \\
\hline 315 & 731.4142 & -0.00631 & -0.00243 & -0.00418 & 0.000465 & 0.001284 & 0.001984 & 0.002449 \\
\hline 316 & 727.6825 & -0.00448 & -0.0036 & -0.00271 & 0.000659 & $3.79 E-05$ & 0.003612 & 0.002328 \\
\hline 317 & 723.9508 & -0.00609 & -0.00211 & -0.0007 & 0.002337 & 0.001633 & 0.004503 & 0.002582 \\
\hline 318 & 720.2191 & -0.00823 & -0.0018 & $-0,0018$ & 0.003083 & 0.003483 & 0.001606 & $-6.5 E-05$ \\
\hline 319 & 716.4873 & -0.01052 & -0.00518 & -0.00618 & -0.00112 & -0.00192 & -0.00196 & -0.00296 \\
\hline 320 & 712.7556 & -0.009 & .0 .00408 & -0.00503 & -0.00335 & -0.00193 & 0.000819 & 0.002041 \\
\hline 321 & 709.0239 & -0.00263 & 0.002016 & $8.46 E 05$ & 0.000322 & 0.006467 & 0.00659 & 0.008253 \\
\hline 322 & 705.2922 & 0.000411 & 0.00198 & 0.000152 & 0.000988 & 0.008183 & 0.007496 & 0.007357 \\
\hline 323 & 701.5605 & -0.00433 & -0.00022 & -0.00489 & 0.000451 & 0.001238 & 0.008372 & 0.003532 \\
\hline 324 & 697.8288 & -0.00972 & 0.001882 & -0.00648 & -0.0021 & -0.00287 & 0.006825 & 0.000633 \\
\hline 325 & 694.0971 & -0.01283 & 0.001633 & -0.00345 & -0.00274 & 0.004553 & 0.003136 & 0.005014 \\
\hline 326 & 690.3654 & -0.00477 & -0.00307 & 0.001101 & 0.00155 & 0.015475 & 0.008684 & 0.007746 \\
\hline 327 & 686.6337 & 0.002714 & 0.004069 & 0.008987 & 0.017488 & 0.023048 & 0.017339 & 0.00774 \\
\hline 328 & 682.902 & 0.002344 & 0.008319 & 0.0125 & 0.019268 & 0.012339 & 0.021648 & 0.014214 \\
\hline 329 & 679.1703 & 0.01973 & 0.009579 & 0.014092 & 0.022671 & 0.006015 & 0.020514 & 0.020884 \\
\hline 330 & 675.4386 & 0.022907 & 0.027308 & 0.011454 & 0.008652 & 0.013533 & 0.00993 & 0.018337 \\
\hline$\frac{331}{332}$ & 671.7069 & 0.040395 & $\begin{array}{l}0.047424 \\
0034306\end{array}$ & $\begin{array}{r}0.04506 \\
-0.0015\end{array}$ & 0.017746 & $\begin{array}{l}0.03161 \\
0.041259\end{array}$ & -0.00108 & 0.038309 \\
\hline 333 & 664.2435 & -0.03673 & 0.031214 & -0.10889 & 0.176551 & -0.01099 & -0.01847 & -0.01638 \\
\hline 334 & 660.5118 & 0.01329 & 0.05025 & 0.07151 & 0.057809 & 0.006968 & 0.009254 & -0.00372 \\
\hline 335 & 656.7801 & 0.045812 & 0.024709 & 0.032092 & 0.047795 & 0.042077 & 0.073839 & 0.0305 \\
\hline 336 & 653.0484 & -0.04397 & 0.004264 & 0.035975 & -0.03612 & -0.01248 & 0.056891 & -0.02779 \\
\hline 337 & 649.3167 & -0.0637 & 0.00249 & -0.01432 & -0.05065 & 0.00042 & 0.118745 & 0.020689 \\
\hline
\end{tabular}


Figure 7-1 Linearity of salicylic acid in pH 7.4 phosphate buffer (corresponds to Figure 2-1 in Chapter 2)

The table below contains the raw data collected during the salicylic acid linearity experiment. Eight serial dilutions of salicylic acid reference standards were used during the linearity study. The molar concentrations are displayed in the table. Standard 1 represents about $21 \mathrm{mg}$ of salicylic acid per $900 \mathrm{ml}$ of buffer while Standard 8 represents about $300 \mathrm{mg}$ of salicylic acid per $900 \mathrm{ml}$ of buffer. Since a large salicylic acid peak was observed at $1388 \mathrm{~cm}^{-1}$, a singlepoint baseline correction at $1370 \mathrm{~cm}^{-1}$ was used to correct the data.

\begin{tabular}{|c|c|c|c|c|}
\hline Description & Flask Volume (L) & Concentration (M) & $\mathbf{1 3 8 8 n m}$ & $\mathbf{1 3 8 8 - 1 3 7 0}$ \\
\hline Std-1 & 0.050 & 0.03215 & 0.01152 & 0.00855 \\
\hline Std-2 & 0.100 & 0.01607 & 0.00580 & 0.00452 \\
\hline Std-3 & 0.150 & 0.01072 & 0.00393 & 0.00324 \\
\hline Std-4 & 0.200 & 0.00804 & 0.00339 & 0.00290 \\
\hline Std-5 & 0.250 & 0.00643 & 0.00288 & 0.00245 \\
\hline Std-6 & 0.300 & 0.00536 & 0.00259 & 0.00215 \\
\hline Std-7 & 0.350 & 0.00459 & 0.00235 & 0.00203 \\
\hline Std-8 & 0.700 & 0.00230 & 0.00103 & 0.00118 \\
\hline
\end{tabular}

*Note:Conc. $\sim 100 \%$ of $300 \mathrm{mg}$ tablet in actual vessel (900ml) dissolution 
Figure 7-2 Linear regression chart for saliyclic acid

The figure below is a linear regression plot for the serial dilutions of salicylic acid reference standards. Since a large salicylic acid peak was observed at $1388 \mathrm{~cm}^{-1}$, a single-point baseline correction at $1370 \mathrm{~cm}^{-1}$ was used to correct the data.

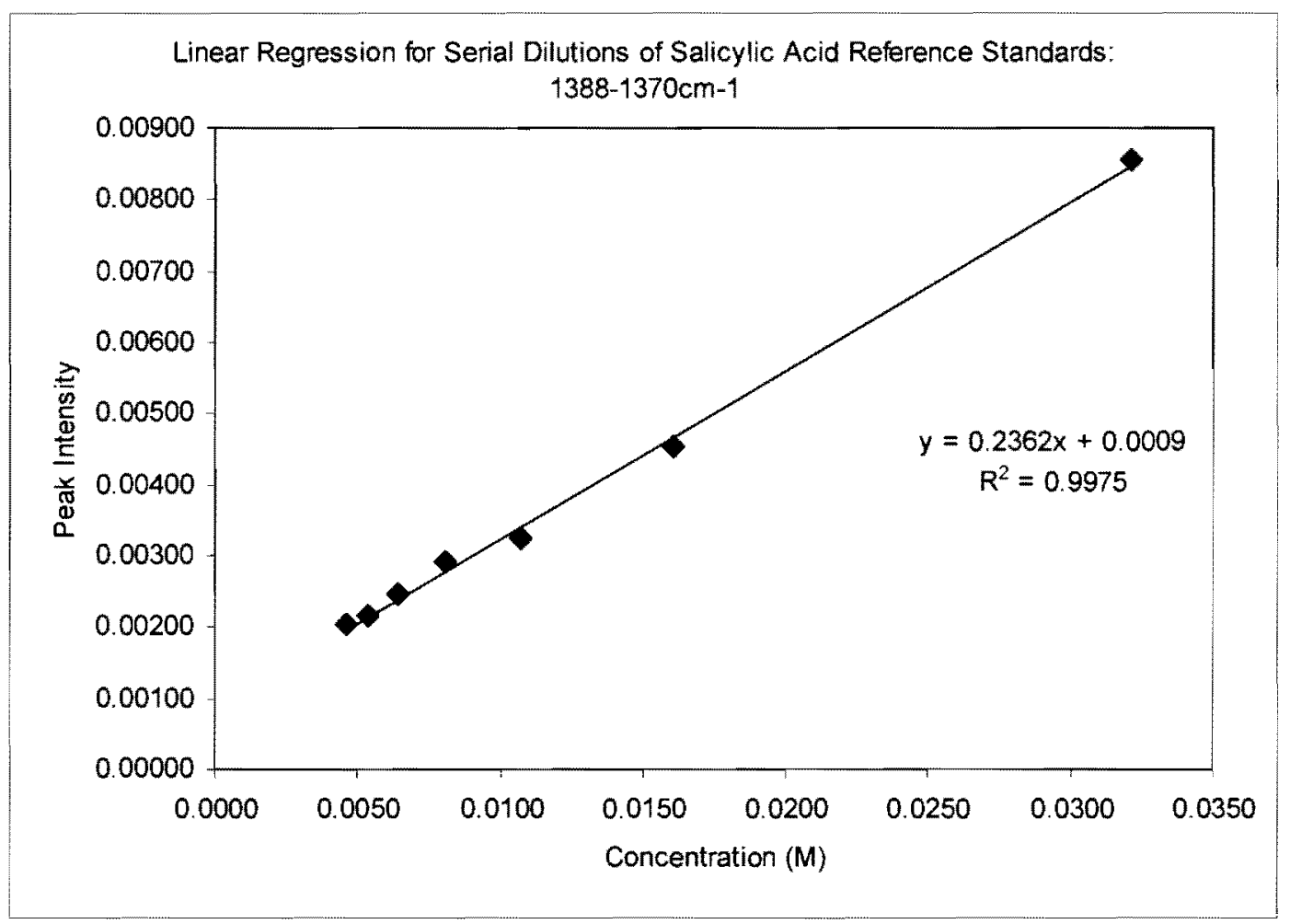


Figure 7-3 Dissolution results for salicylic acid in pH 7.4 phosphate buffer: HPLC vs. IR data (corresponds to Figure 2-2 in Chapter 2)

The table below corresponds to the percent $(\%)$ dissolved data obtained from the salicylic acid dissolution experiment. The table compares ATR-IR to HPLC percent dissolved results. HPLC samples were collected after $30 \mathrm{~min}, 1$ hour, 2 hour, 3 hour, 6.5 hours and finally after 7 hours. Moreover, the samples were analyzed using an Agilent HPLC system. As shown in the table below the maximum absolute difference observed between the IR and HPLC data was found to be $3 \%$.

\begin{tabular}{|c|c|c|c|}
\hline Time (hh:mm) & Dissolved (HPLC) & Dissolved by (IR) & Difference \\
\hline $00: 00$ & $0.0 \%$ & $0.0 \%$ & $0 \%$ \\
\hline $00: 30$ & $11.1 \%$ & $14.4 \%$ & $-3 \%$ \\
\hline $01: 00$ & $20.9 \%$ & $23.1 \%$ & $-2 \%$ \\
\hline $02: 00$ & $38.2 \%$ & $38.0 \%$ & $0 \%$ \\
\hline $03: 00$ & $52.9 \%$ & $50.9 \%$ & $2 \%$ \\
\hline $06: 30$ & $87.8 \%$ & $88.3 \%$ & $-1 \%$ \\
\hline $07: 00$ & $94.8 \%$ & $97.8 \%$ & $-3 \%$ \\
\hline
\end{tabular}


Figure 7-4 ATR-IR System photo

The figure below shows a photo of the Mettler Toledo ATR-IR system coupled with a DiComp fiber optic probe. The photo shows the DiComp probe inserted into a standard dissolution vessel. An external electric stirrer was used to control the agitation speed (RPM's).

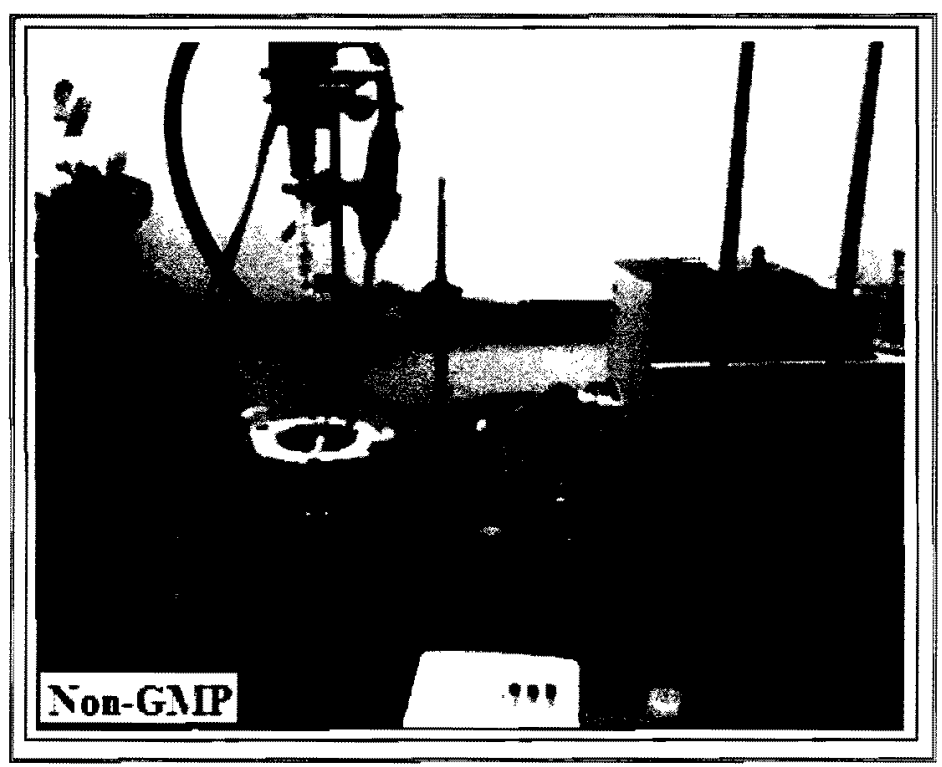




\section{Figure 7-5 Mettler Toledo ATR-IR/ Dissolution Systems}

The figure below shows a photo of the Mettler Toledo ATR-IR system coupled with a DiComp fiber optic probe. The photo shows the DiComp probe inserted into a standard dissolution vessel. The Vankel six-vessel dissolution system was used for during testing.

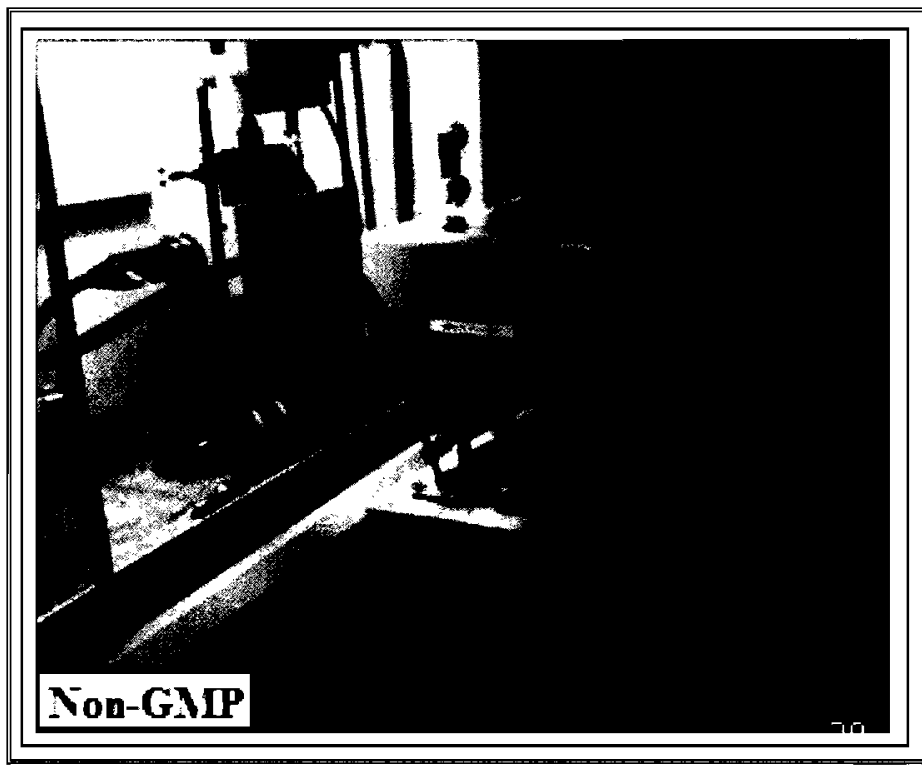


Figure 7-6

Flow Cell Fiber Optic Probe:

The figure below shows a photo of the Mettler Toledo ATR-IR system coupled with a Flow Cell fiber optic probe. Plumbing from the Flow Cell was attached to an Agilent six-port valve pump. Also, plumbing from a dissolution vessel was attached to the pump. Thus, a close-loop system was created whereby dissolution samples were drawn to the flow cell via the pump and back to the dissolution vessel. Homogeneous mixing, within the dissolution vessel, was achieved using a stir plate and magnetic stir bar.

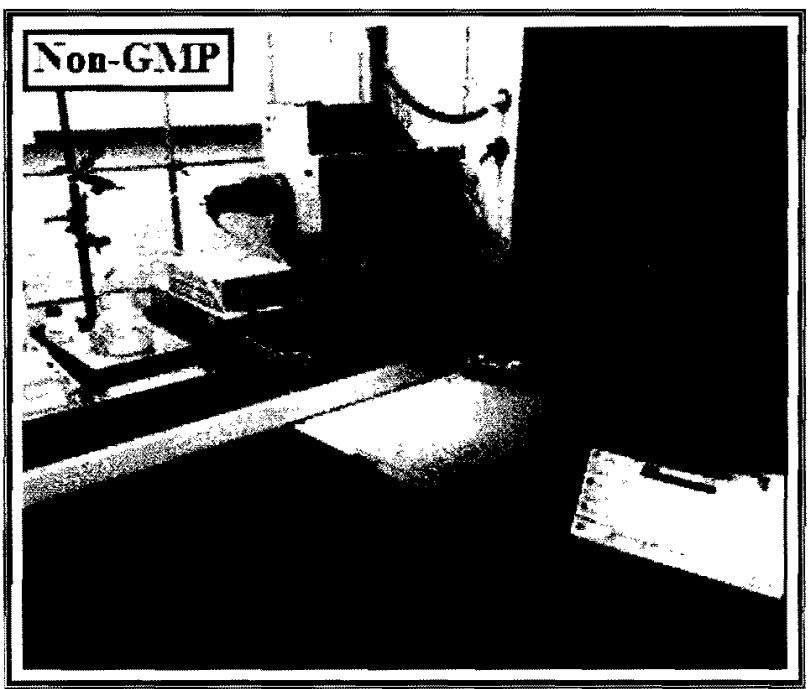




\section{Figure 7-7 K4 Fiber Optic Probe}

The figure below shows a photo of the Mettler Toledo ATR-IR system coupled with a K4 Conduit fiber optic probe. The photo also shows a dissolution vessel attached to the K4 probe. The dissolution vessel was produced out of a methanol bottle. A valve was attached to the vessel in order to empty the vessel contents during dissolution runs and cleaning.

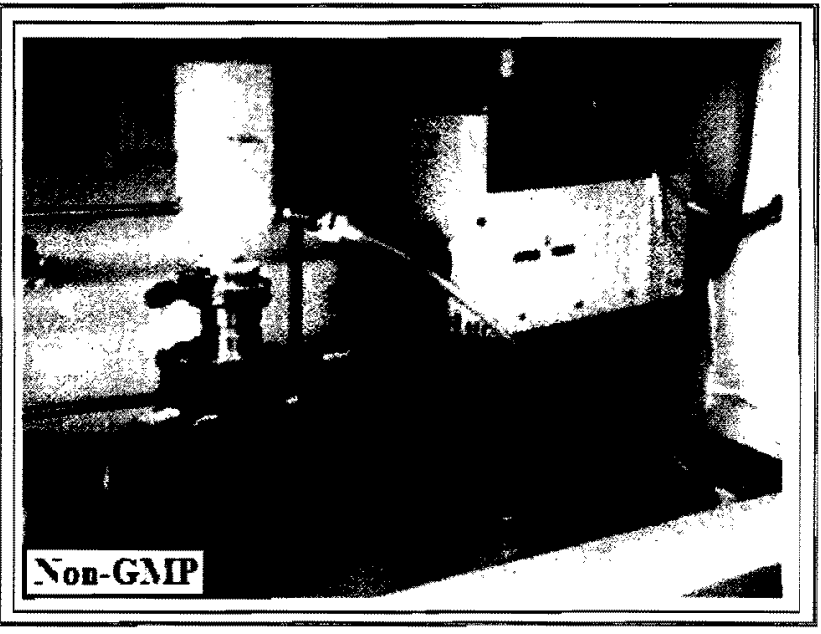


Figure 7-8 HPLC analysis for Salicylic Acid calibrator tablets

Salicylic Acid Tablets (HPLC Analysis)

Batch No: Salicylic Acid calibrator tablets Lot \# Q00200

\begin{tabular}{|c|c|c|c|c|c|}
\hline Sample Name & Vial No & Injection & Result ID & RT & Area \\
\hline Standard 1 & 1 & 1 & 2533 & 0.7 & 4252948 \\
\hline $30 \mathrm{~min}$ & 2 & 1 & 2534 & 0.7 & 472913 \\
\hline 2 hour & 4 & 1 & 2536 & 0.7 & 1629150 \\
\hline 3 houf & 5 & 1 & 2537 & 0.7 & 2259379 \\
\hline Standard 1 & 1 & 1 & 2533 & 0.7 & 4252948 \\
\hline
\end{tabular}

Standard-1 Weight (mg): $\quad 60.01$ Standard Declared Content (\%): $\quad 100.0$

Vessel Volume $(\mathrm{mL})$ Potency (mg)

Standard Volume (mL) Sample $(\mathrm{mL})$
500.0

300.0

1000

1.5

\begin{tabular}{|c|c|}
\hline Time (hour) & Vessel Volume \\
\hline 0.5 & 500.0 \\
\hline 1 & 498.5 \\
\hline 2 & 497.0 \\
\hline 3 & 495.5 \\
\hline 6.5 & 494.0 \\
\hline 7 & 492.5 \\
\hline
\end{tabular}

Standard-1 Avg Area Std-1Wt/Std-1PA:

4252948

1.411.E-05

\begin{tabular}{|c|c|}
\hline Time (hour) & \% Dissolved (HPLC) \\
\hline 0.5 & $11 \%$ \\
\hline 1 & $21 \%$ \\
\hline 2 & $38 \%$ \\
\hline 3 & $53 \%$ \\
\hline 6.5 & $88 \%$ \\
\hline 7 & $95 \%$ \\
\hline
\end{tabular}


Figure 7-9 HPLC Method Parameters: Single Component and Multi Component Experiments

HPLC Parameters (HP1050)

- Symmetry $300 \mathrm{C} 1857 \mathrm{~m}$ column $(4.6 \mathrm{~mm} \times 50 \mathrm{~mm})$.

* Mobile phase: 60/40/1 (water/methanol/glacial acetic acid),

* Flow rate: $2.0 \mathrm{ml} / \mathrm{min}$, isocratic mode. Injection volume $=5 \mu \mathrm{L}$

- UV detector was set at $296 \mathrm{~nm}$

\section{HPLC Parameters (Waters 2695)}

- Symmetry Shied RP C18 3um column $(3.5 \mathrm{~mm} \times 50 \mathrm{~mm})$

" Mobile phase: 70/30/1 (water/acetonitrile/trifluoroacetic acid (TFA)),

- Flow rate: $1.5 \mathrm{ml} / \mathrm{min}$, isocratic mode, injection volurne was $5 \mu l$. UV detection was set at $296 \mathrm{~nm}$.

\section{HPLC Parameters (HP1050)}

* Phenomenex Intersil ODS C18 $5 \mu \mathrm{m}$ column $(4.6 \mathrm{~mm} \times 150 \mathrm{~mm})$,

- Mobile phase: 60/40/1 (methanol/water/trifluoroacetic acid (TFA)).

* Flow rate: $1.0 \mathrm{~m} / \mathrm{min}$, isocratic mode, injection volume : $25 \mu \mathrm{l}$

- UV detection was set at $280 \mathrm{~nm}$. 
Figure 7-10 Typical Excedrin® experiment spectra

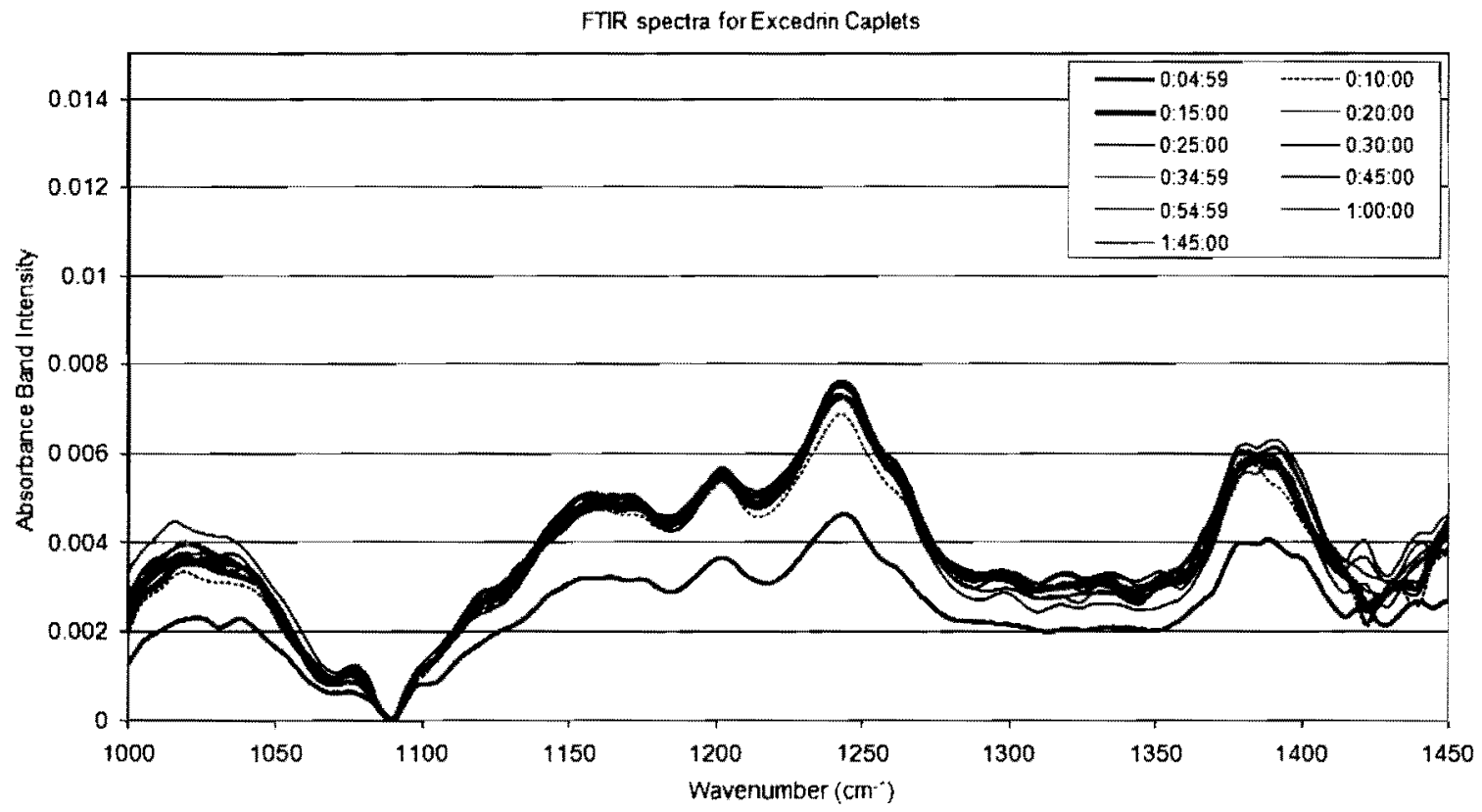

Figure 7-11 Excedrin® HPLC Data

$\begin{array}{ccc} & \text { Ret time (min) } & \text { Area } \\ \text { Acetaminophen } & 2.033 & 19015.8 \\ \text { AcetylSalicylic Acid } & 3.585 & 9004.3 \\ & & \\ & \text { Acetaminophen } & \text { Acetylsalicylic Acid } \\ 10 \mathrm{~min} & 15856.1 & 8684.1 \\ 20 \mathrm{~min} & 16144.8 & 8848.6 \\ 30 \mathrm{~min} & 18301.0 & 9224.6 \\ 1 \text { hour } & 18868.4 & 9341.3 \\ & & \\ 0 & \% \text { Acetaminophen } & \text { Acetylsalicylic Acid } \\ 10 & 0 & 0 \\ 20 & 83.4 & 96.4 \\ 30 & 84.1 & 97.3 \\ 60 & 94.3 & 100.4 \\ & 96.2 & 100.6\end{array}$




\section{Appendix [B]: Studying drug kinetics via ATR-IR Spectroscopy}

Figure 8-1 Salicylic Acid standard spectra

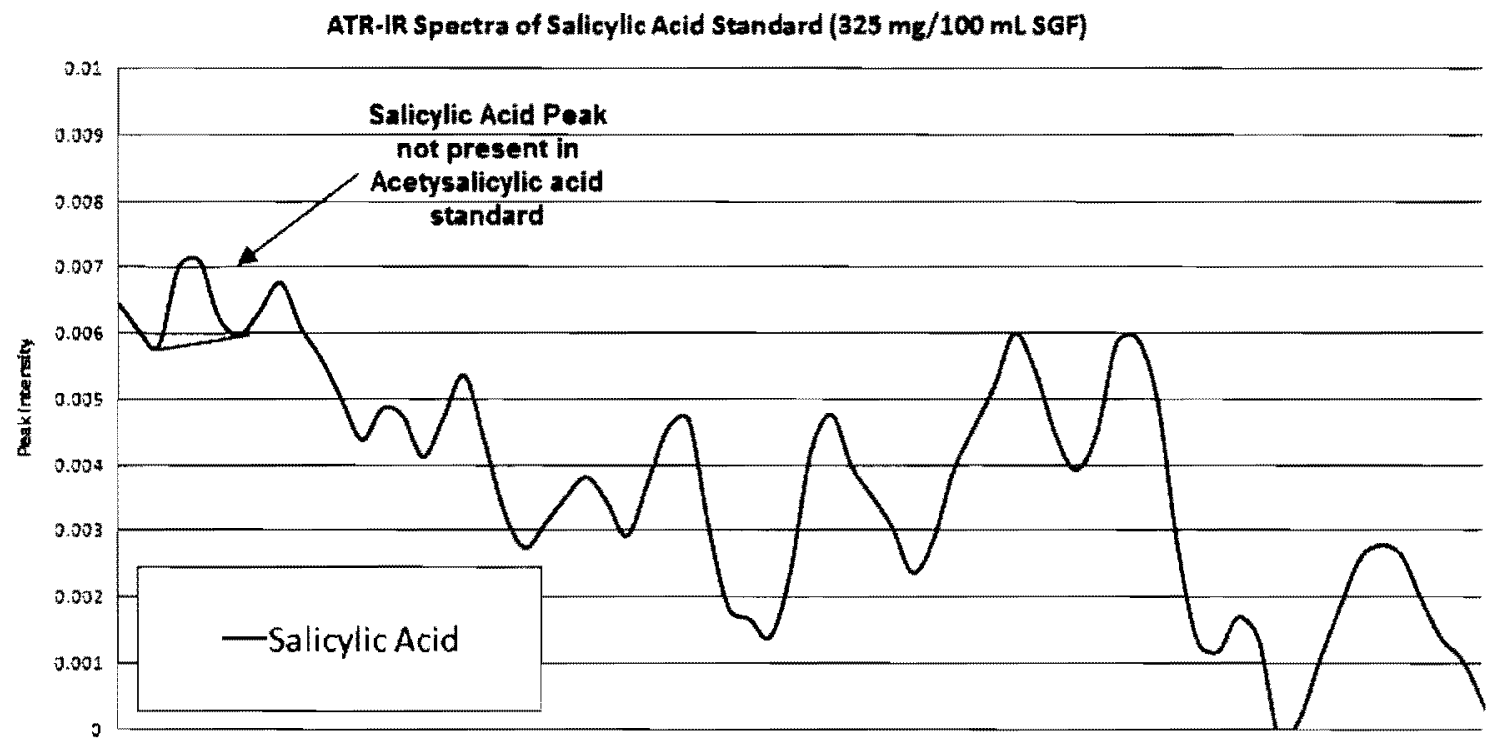

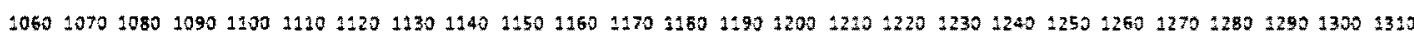

Wavenumber $(\mathrm{sm} \cdot 1)$ 
Seton Hall University

Figure 8-2 Acetylsalicylic Acid standard spectra

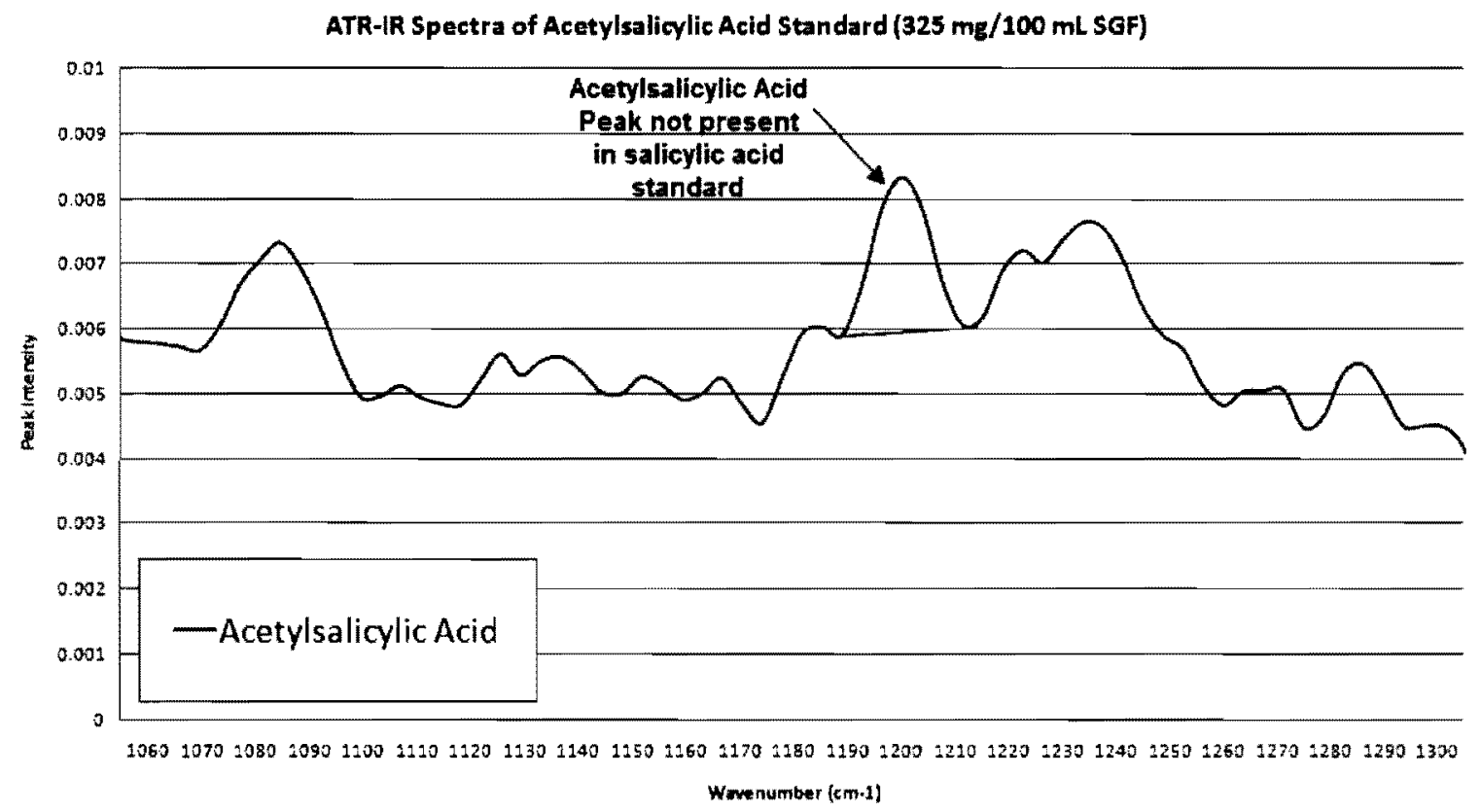


Seton Hall University

Table 8-1 Aspirin FT-IR Frequencies of Interest

\begin{tabular}{|l|l|}
\hline Wavelength (c-m-') & Assignment \\
\hline $2300-2500$ & carboxyl $\mathrm{OH}$ \\
\hline 1760 & vinyl ester $\mathrm{C}=\mathrm{O}$ \\
\hline 1690 & aromatic acid $\mathrm{C}=\mathrm{O}$ \\
\hline 1610 & aromatic acid $\mathrm{C}=\mathrm{O}$ \\
\hline 1580 & aromatic $\mathrm{C}=\mathrm{C}$ stretch \\
\hline 1490 & aromatic $\mathrm{C}=\mathrm{C}$ stretch \\
\hline 1220 & $=\mathrm{C}-0$ (acid and ester) \\
\hline 1190 & $=\mathrm{C}-0$ (acid and ester) \\
\hline 760 & ortho substituted phenyl $\mathrm{C}-\mathrm{H}$ bending \\
\hline
\end{tabular}


Figure 8-3 Rate Order Formulas and Equations (Reproduced from Wikipedia website on Rate Order)

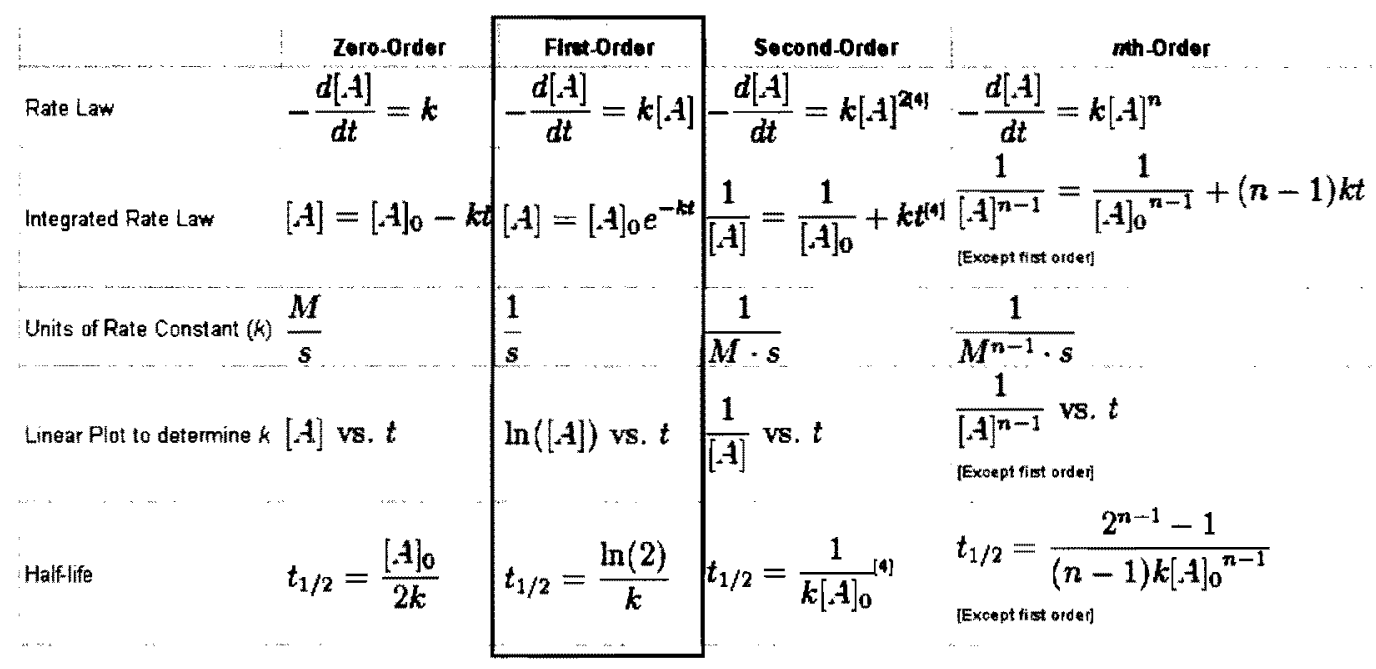




\section{Appendix [C]: New Instrument Design ("Solv-IR")} attachments

Figure 9-1

ATR-IR Technique Diagram (courtesy of Jian Wang, Ph.D., Mettler Toledo)

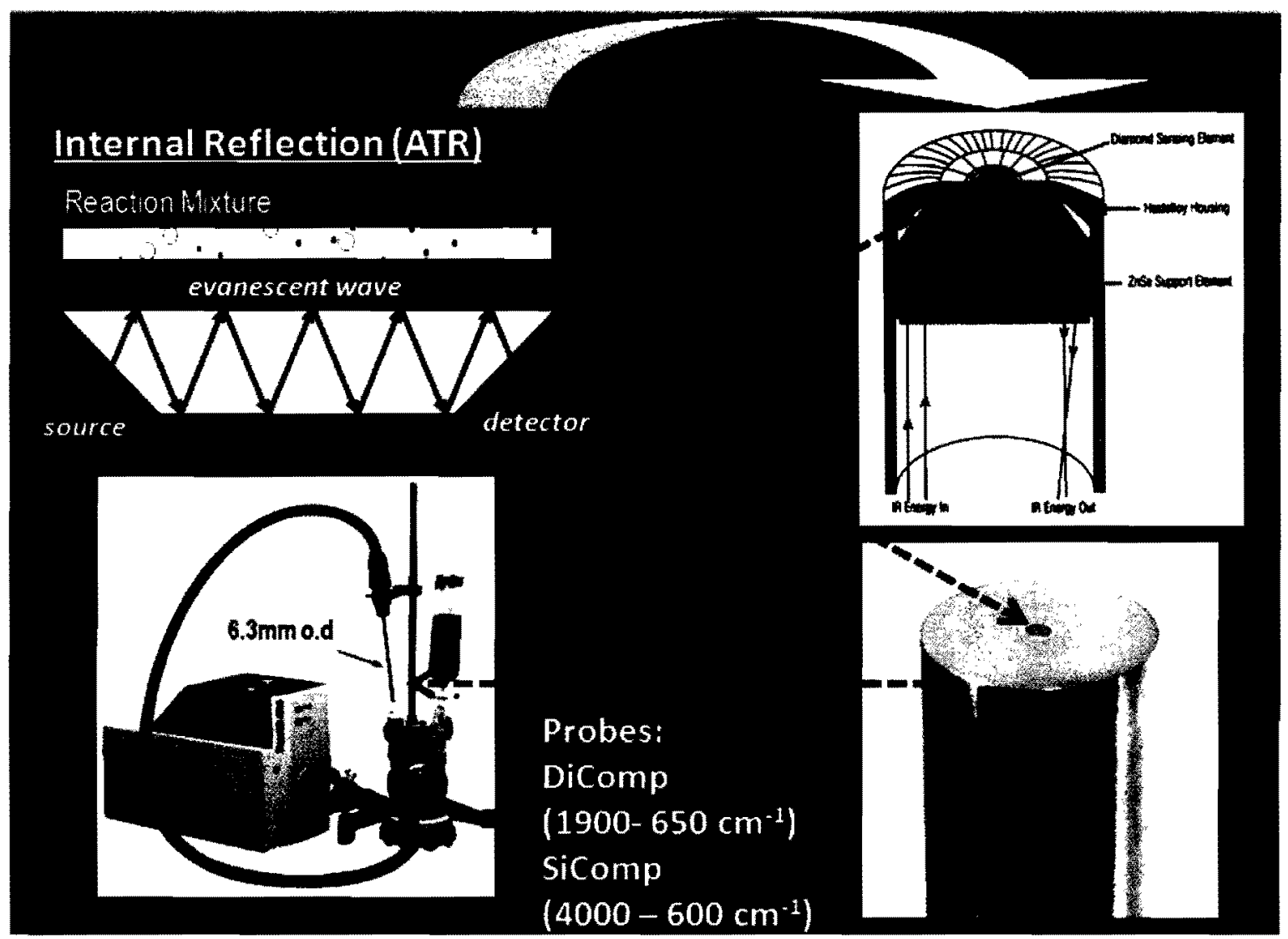


10 Appendix [D]: BP Gulf oil research attachments

Figure 10-1 Detergents used during BP Gulf oil research

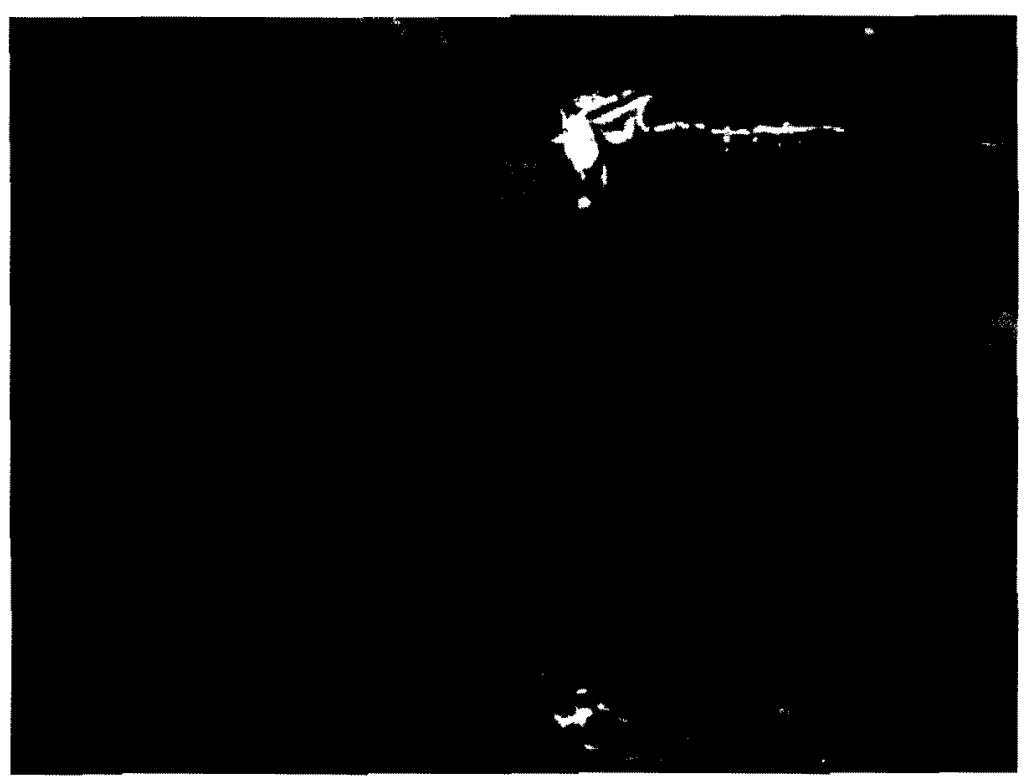


Figure 10-2 IR Spectra of Gulf Oil from Riser: Pre- April 20, 2010

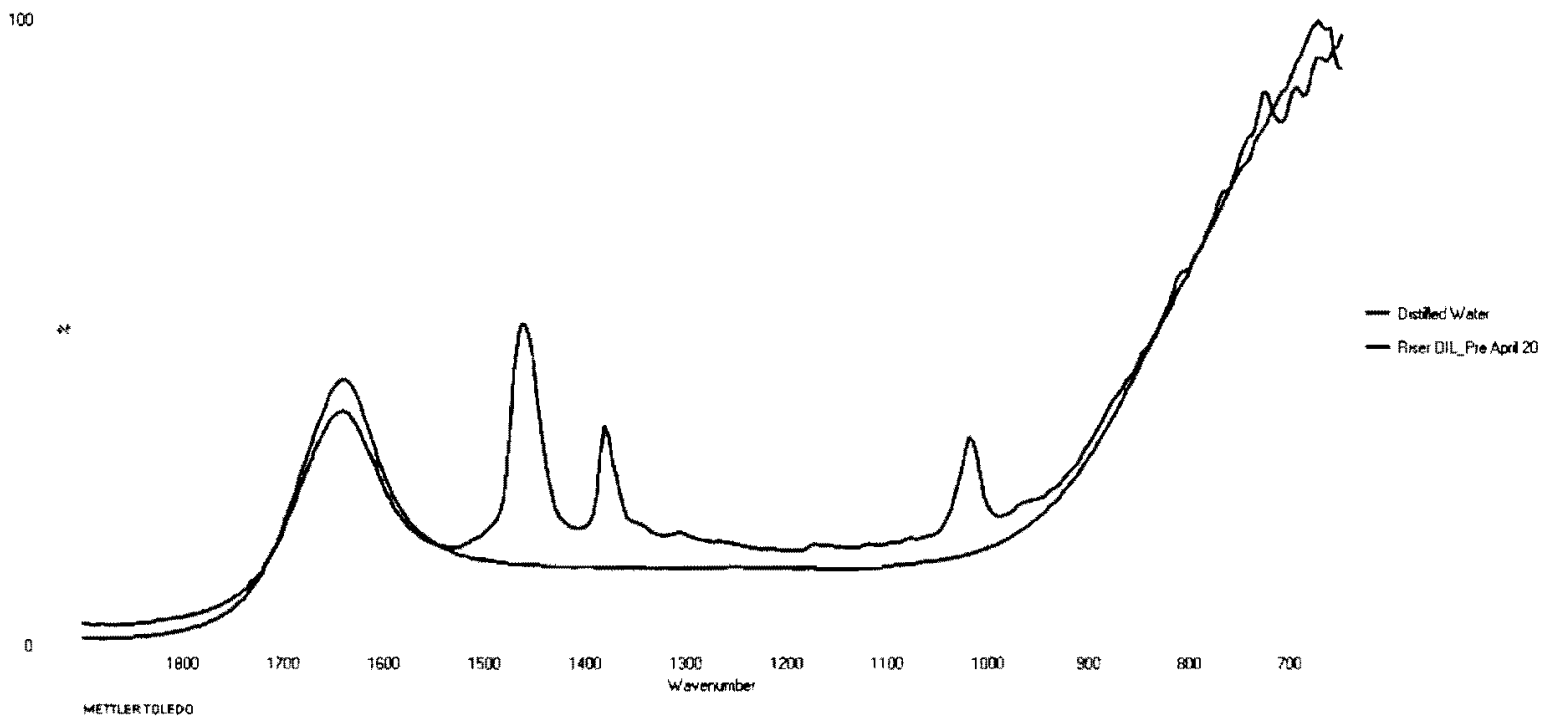


Seton Hall University

Table 10-1 Amount of Dawn® dishwashing detergent added

\begin{tabular}{|c|c|c|}
\hline Time (h:mm) & Volume added $(\mathbf{m l})$ & Total volume in vessel $(\mathbf{m l})$ \\
\hline $0: 01$ & 1 & 2 \\
\hline $0: 04$ & 1 & 3 \\
\hline $0: 06$ & 1 & 6 \\
\hline $0: 08$ & 3 & 9 \\
\hline $0: 10$ & 3 & 12 \\
\hline $0: 13$ & 3 & 15 \\
\hline $0: 14$ & 3 & 18 \\
\hline $0: 17$ & 3 & 21 \\
\hline $0: 19$ & 3 & 24 \\
\hline $0: 20$ & 3 & 27 \\
\hline $0: 22$ & 3 & 30 \\
\hline $0: 24$ & 3 & 33 \\
\hline $0: 26$ & 3 & 36 \\
\hline $0: 26$ & 3 & 39 \\
\hline $0: 28$ & 3 & 42 \\
\hline $0: 34$ & 3 & 45 \\
\hline $0: 39$ & 3 & 48 \\
\hline $0: 41$ & 3 & 51 \\
\hline $1: 48$ & 3 & 54 \\
\hline $2: 06$ & 3 & \\
\hline & & \\
\hline
\end{tabular}


Figure 10-3 Thermo Nicolet 4700: \% Transmittance of OIL samples
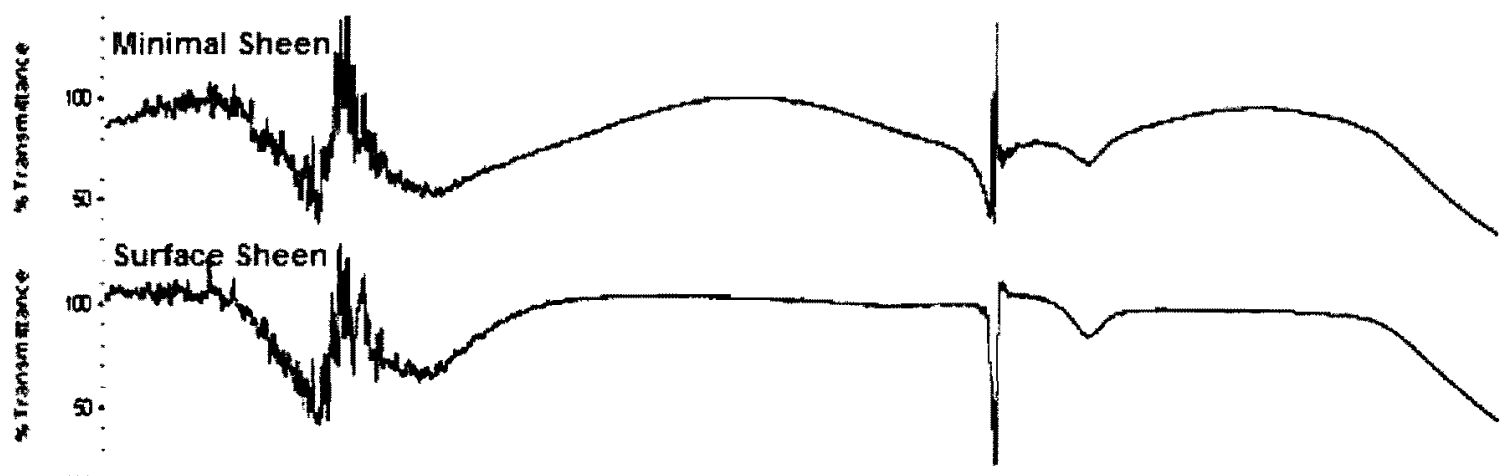

2000
$\vdots$
5
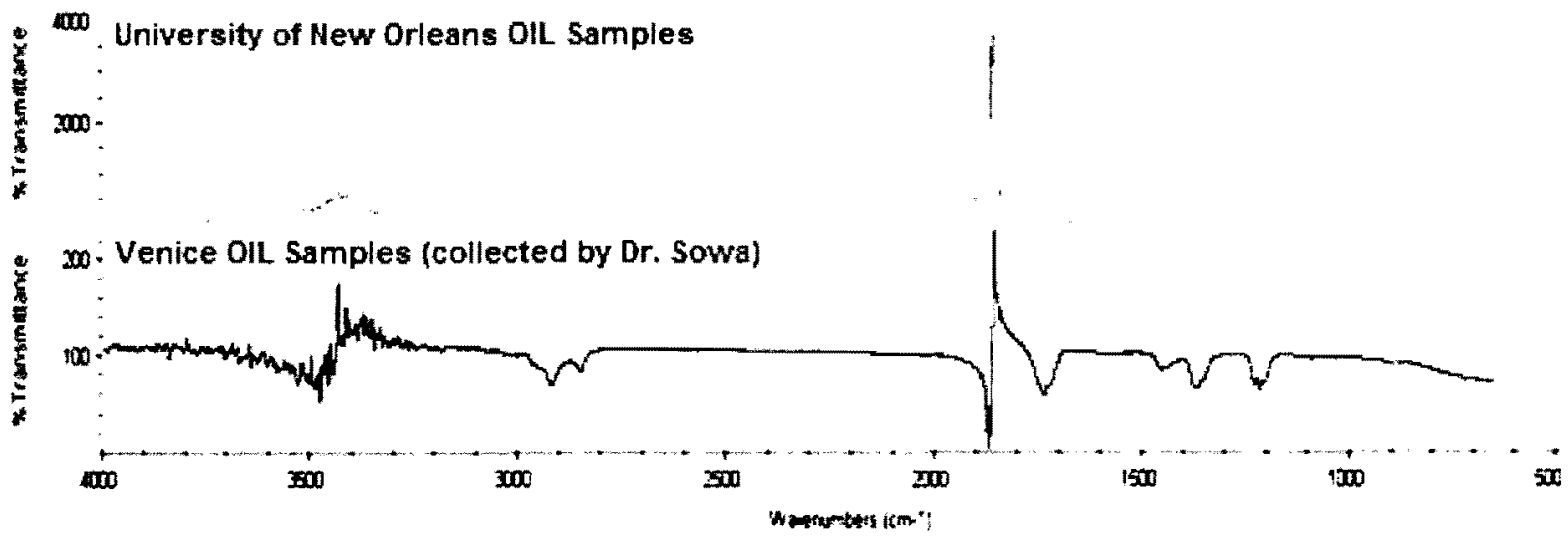
Seton Hall University

Figure 10-4 Thermo Nicolet 4700: Absorbance of OIL samples
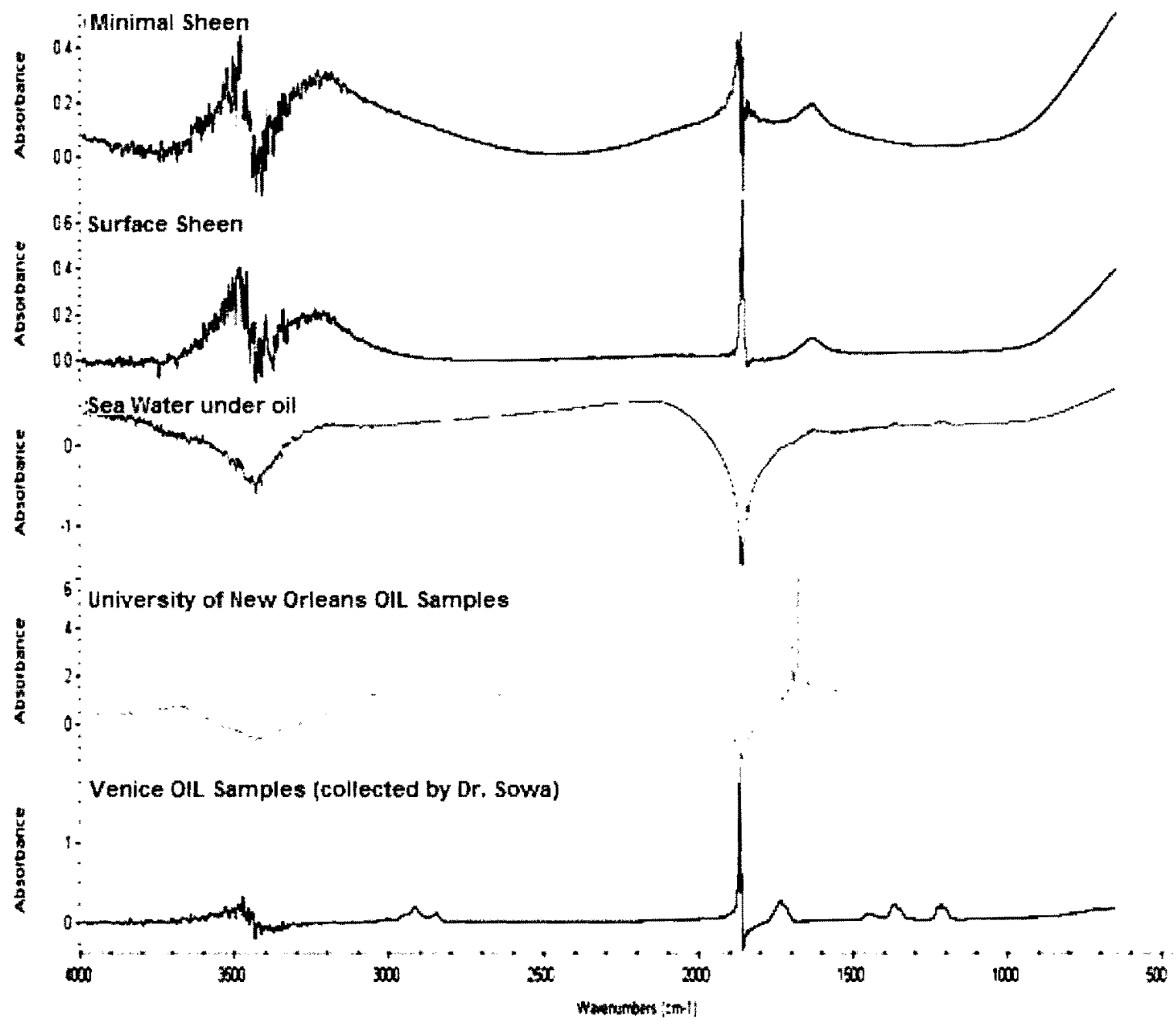